\section{Pacific Northwest}

National Laboratory

Operated by Battelle for the

U.S. Department of Energy

\title{
Characterization of Direct Push Vadose Zone Sediments from the 241-U Single-Shell Tank Farm
}

\author{
C. F. Brown \\ M. M. Valenta \\ R. J. Serne \\ B. N. Bjornstad \\ D. C. Lanigan \\ C. Iovin \\ R. E. Clayton
}

December 2007

\author{
K. N. Geiszler \\ E. T. Clayton \\ I. V. Kutnyakov \\ S. R. Baum \\ M. J. Lindberg \\ R. D. Orr
}

Prepared for the U.S. Department of Energy
under Contract DE-AC05-76RL01830 


\title{
DISCLAIMER
}

This report was prepared as an account of work sponsored by an agency of the United States Government. Neither the United States Government nor any agency thereof, nor Battelle Memorial Institute, nor any of their employees, makes any warranty, express or implied, or assumes any legal liability or responsibility for the accuracy, completeness, or usefulness of any information, apparatus, product, or process disclosed, or represents that its use would not infringe privately owned rights. Reference herein to any specific commercial product, process, or service by trade name, trademark, manufacturer, or otherwise does not necessarily constitute or imply its endorsement, recommendation, or favoring by the United States Government or any agency thereof, or Battelle Memorial Institute. The views and opinions of authors expressed herein do not necessarily state or reflect those of the United States Government or any agency thereof.

\author{
PACIFIC NORTHWEST NATIONAL LABORATORY \\ operated by \\ BATTELLE \\ for the \\ UNITED STATES DEPARTMENT OF ENERGY \\ under Contract DE-AC05-76RL01830
}

Printed in the United States of America

Available to DOE and DOE contractors from the

Office of Scientific and Technical Information,

P.O. Box 62, Oak Ridge, TN 37831-0062;

ph: (865) 576-8401

fax: (865) 576-5728

email: reports@adonis.osti.gov

\author{
Available to the public from the National Technical Information Service, \\ U.S. Department of Commerce, 5285 Port Royal Rd., Springfield, VA 22161 \\ ph: (800) 553-6847 \\ fax: $(703) 605-6900$ \\ email: orders@ntis.fedworld.gov \\ online ordering: http://www.ntis.gov/ordering.htm
}

This document was printed on recycled paper.

(9/2003) 


\section{Characterization of Direct Push Vadose Zone Sediments from the 241-U Single-Shell Tank Farm}
C. F. Brown
K. N. Geiszler
M. M. Valenta
E. T. Clayton
R. J. Serne
I. V. Kutnyakov
B. N. Bjornstad
S. R. Baum
D. C. Lanigan
M. J. Lindberg
C. Iovin
R. D. Orr
R. E. Clayton

December 2007

Prepared for CH2M HILL Hanford Group, Inc. and the U.S. Department of Energy under Contract DE-AC05-76RL01830

Pacific Northwest National Laboratory

Richland, Washington 99352 



\section{Executive Summary}

The overall goals of the Tank Farm Vadose Zone Project, led by CH2M HILL Hanford Group, Inc., are 1) to define risks from past and future single-shell tank farm activities, 2) to identify and evaluate the efficacy of interim measures, and 3) to aid, via collection of geochemical information and data, the future decisions that must be made by the U.S. Department of Energy (DOE) regarding the near-term operations, future waste retrieval, and final closure activities for the single-shell tank Waste Management Areas (WMAs). For a more complete discussion of the goals of the Tank Farm Vadose Zone Project, see the overall work plan, Phase 1 RCRA Facility Investigation/Corrective Measures Study Work Plan for the Single-Shell Tank Waste Management Areas (DOE 1999). Specific details on the rationale for activities performed at WMA U are found in Crumpler (2003). To meet these goals, CH2M HILL Hanford Group, Inc., asked scientists from Pacific Northwest National Laboratory (PNNL) to perform detailed analyses of vadose zone sediment collected within the U Single-Shell Tank Farm. Specifically, this report contains all the geochemical and selected physical characterization data collected on vadose zone sediment recovered from ten direct push characterization holes emplaced to investigate vadose zone contamination associated with potential leaks within the 241-U Single-Shell Tank Farm. Specific tanks targeted during this characterization campaign included tanks 241-U-104/241-U-105, 241-U-110, and 241-U-112. Additionally, this report compiles data from direct push samples collected north of tank 241-U-201, as well as sediment collected from the background borehole (C3393).

The U Tank Farm geochemical investigation was performed using pairs of direct push probe holes. A total of 20 direct pushes were driven within the U Tank Farm; 10 of these holes were logged for moisture, gross gamma, and in some cases spectral gamma using calibrated probes and ten were driven for the purpose of retrieving vadose zone sediment for characterization and analysis. The locations of the direct pushes were chosen to investigate an estimated 50,000 gallon leak of bismuth phosphate metals waste from tank 241-U-104, small leaks of reduction oxidation process (REDOX) supernatant from tanks 241U-110 and 241-U-112, a 30,000 gallon leak of waste from tank 241-U-101 (as yet unconfirmed from measurements in the vadose zone), and a resistivity anomaly near the U Tank Farm 200 series tanks.

A core log was generated for all samples and a visual geologic evaluation of all liner samples was performed at the time of sample processing. Aliquots of sediment from the liners were analyzed and characterized in the laboratory for the following parameters: moisture content, gamma-emitting radionuclides, one-to-one sediment:water extracts (which provided soil $\mathrm{pH}$, electrical conductivity, cation, trace metal, and anion data), total carbon and inorganic carbon content, and $8 \mathrm{M}$ nitric acid leaches (which provided a measure of the total leachable contaminant content in the sediment). Concentrations of two key radioactive contaminants, technetium-99 and uranium-238, along with other trace metals, were determined in acid and water extracts using inductively coupled plasma-mass spectrometry (ICP-MS). All of the parameters were elevated in at least some of the samples analyzed as part of this study.

After evaluating all the characterization and analytical data, there is no question that the vadose zone in the vicinity of tanks $241-\mathrm{U}-104$ and $241-\mathrm{U}-105$ has been contaminated by tank-related waste. This observation is not new, as gamma logging of drywells in the area has identified uranium contamination at the same depths interrogated by push hole C5602. Given that the deepest sample string analyzed from push hole C5602 contained trace activities of technetium-99, it is obvious that tank waste contamination has impacted the vadose zone to at least a depth of $92 \mathrm{ft}$ bgs at this location. However, the scope of the sampling campaign was to acquire additional samples to better understand the extent of contamination in 
the U Tank Farm; therefore, future characterization activities (i.e., a borehole) will be required to understand the total vertical depth of contamination at this location.

The vadose zone south tank $241-\mathrm{U}-110$ has also been affected by a tank-related waste solution. The presence of sodium as the dominant water-extractable cation indicates that a high sodium-bearing waste stream has created a cation exchange front in this region that has pushed the common divalent alkaline earth cations (calcium and magnesium) off the surface exchange sites. The presence of significantly elevated concentrations of technetium-99 and nitrate in the deepest samples collected indicate that the vadose zone has been impacted to at least a depth of $98 \mathrm{ft}$ bgs. Given the high soil $\mathrm{pH}$, coupled with the presence of mobile contaminants deep in the vadose zone, it is clear that a release from tank 241-U-110 is the source of contamination intercepted by push hole C5608. Again, the total vertical extent of contamination at this location can not be derived from the direct push sampling results.

Of the remaining direct push samples analyzed, only two contained quantifiable or elevated concentrations of mobile tank waste contaminants. Samples from push hole C5600, which was emplaced southwest of tank 241-U-105, contained a quantifiable activity of technetium-99 in the acid extract of the sample collected from $88 \mathrm{ft}$ bgs. Unfortunately, this result was not corroborated by the water extract results, which failed to detect technetium-99 at a concentration above the sample estimated limit of quantification. While this sole data point should not be dismissed as an outlier, it is clear that additional data should be collected prior to assessing or confirming the level of contamination southwest of tank 241-U-105.

Two samples collected from push hole C5606 (emplaced northeast of tank 241-U-112) contained slightly elevated concentrations of acid-extractable uranium. Tank 241-U-112 was estimated to have released only $24 \mathrm{~kg}$ of uranium to the vadose; therefore, the lack of significant uranium contamination in a push hole emplaced near the tank is not an unreasonable finding. As with the data collected southwest of tank 241-U-105, these data points should not be considered conclusive evidence of the presence of tank waste in the vadose zone near tank 241-U-112.

Aside from elevated concentrations of water and acid extractable sodium in most (water extracts) or all (acid extracts) of the samples analyzed, no other tank waste constituents were observed at elevated concentrations in the push holes emplaced northeast of tank 241-U-101 or north of the 200 series tanks. Additionally, the inferred porewater chemistry, based on water extracts of the samples collected from push hole C5604, does not support the resistivity anomaly observed by Rucker et al. (2006) north of the 200 series tanks. 


\section{Acknowledgments}

This work was conducted as part of the Tank Farm Vadose Zone Project led by CH2M HILL Hanford Group, Inc., in support of the U.S. Department of Energy (DOE)'s Office of River Protection. The authors wish to thank John G. Kristofzski, Fredrick M. Mann, David A. Myers, Michael P. Connelly, and Harold A. Sydnor with CH2M HILL Hanford Group, Inc., and Dwayne Crumpler with Columbia Energy and Environmental Services for their planning support and technical review of this work, and Wooyong Um, with Pacific Northwest National Laboratory (PNNL), for his technical review of the report. We would also like to express our gratitude to Robert Lober with the DOE Office of River Protection for his support and interest.

We would especially like to thank Kent D. Reynolds, Dave Skoglie, Kelly Olson, and Mark Repko (Duratek Federal Services, Inc.) for their efforts in executing the field work that obtained the samples.

Finally, the authors would also like to thank Theresa Gilbride (PNNL) for completing the editorial review and final formatting of this technical report. 


\section{Contents}

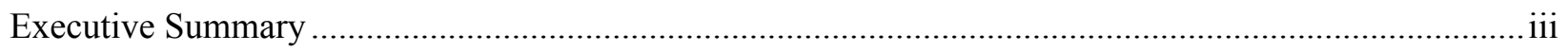

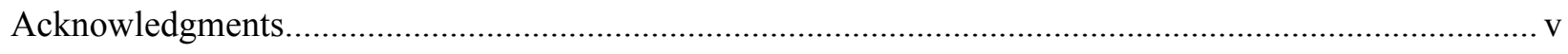

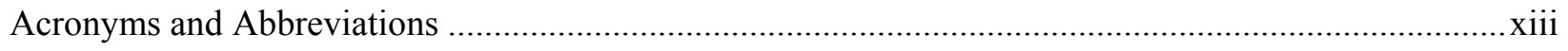

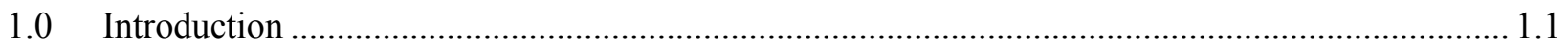

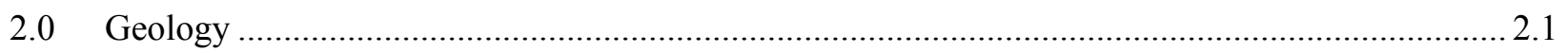

2.1 Stratigraphy of the Vadose Zone Beneath the U Tank Farm ........................................... 2.1

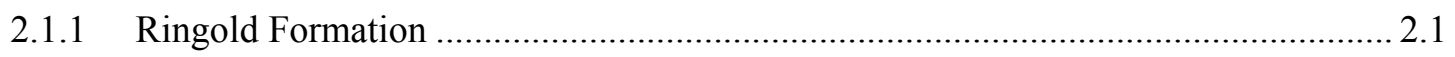

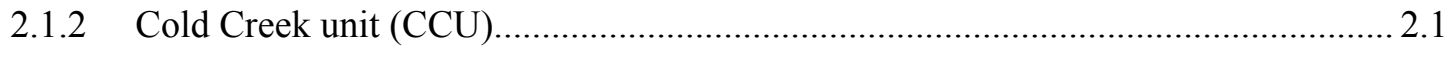

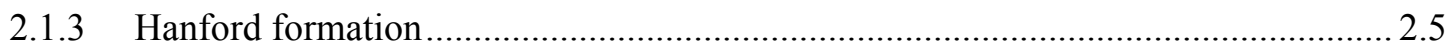

2.2 Interpreted Geology of U Tank Farm Direct-Push Boreholes........................................ 2.10

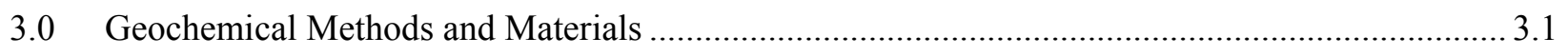

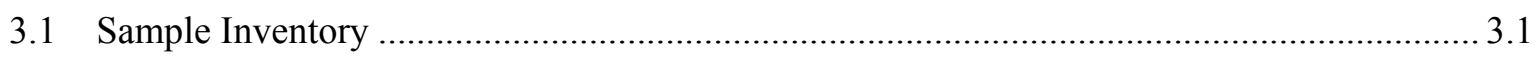

3.1.1 Background Borehole (C3393) Vadose Zone Samples............................................ 3.1

3.1.2 U Tank Farm Direct Push Samples …….............................................................. 3.1

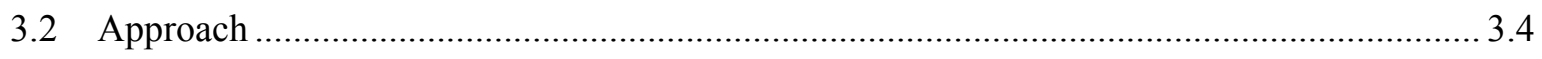

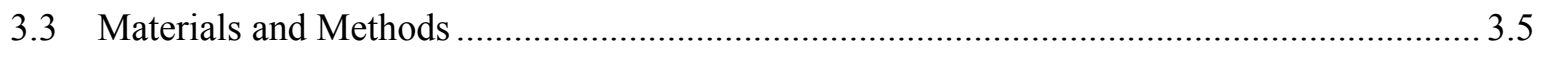

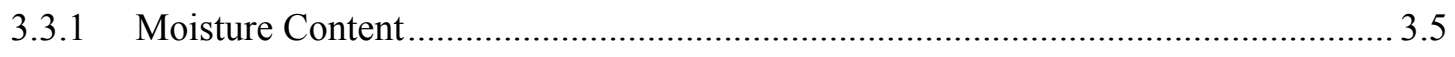

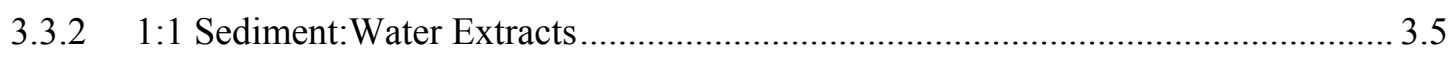

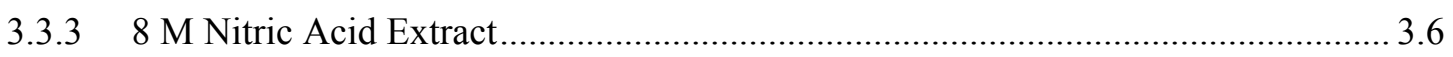

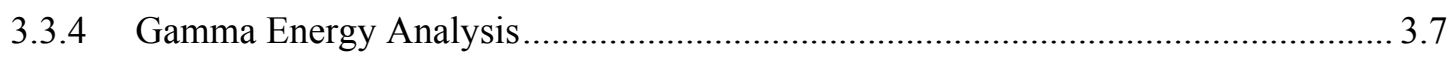

3.3.5 Total Beta and Total Alpha Measurements on Water and Acid Extracts.................. 3.7

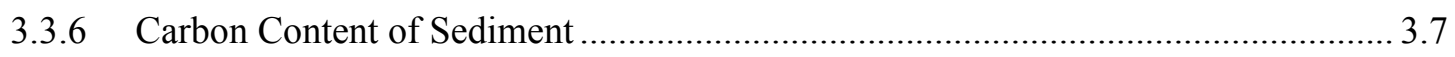

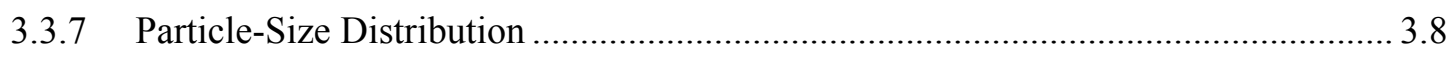

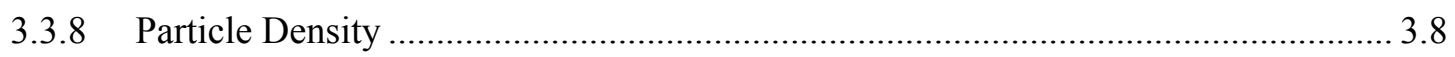

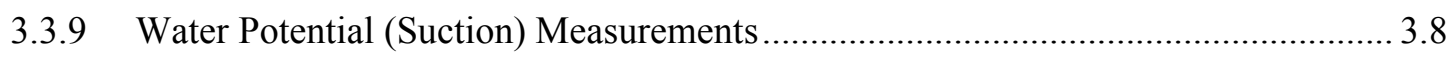

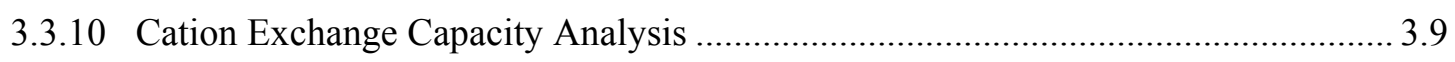

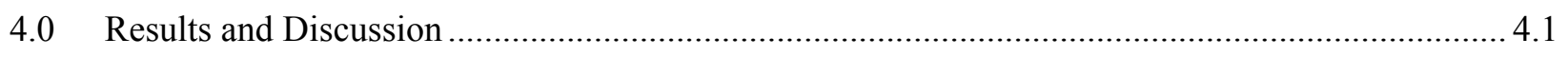

4.1 Vadose Zone Sediment from the Background Borehole Samples (C3393) ......................... 4.1

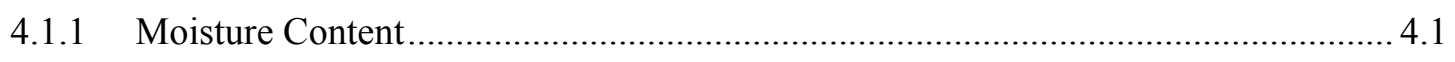

4.1.2 1:1 Sediment:Water Extracts of Sediments from Borehole C3393 .......................... 4.4

4.1.3 Vadose Zone Porewater Chemical Composition in the Background Borehole......... 4.8

4.1.4 8 M Nitric Acid-Extractable Amounts of Selected Elements in the U Tank Farm Direct Push Sediments ............................................................................... 4.11 
4.1.5 Radionuclide Content in Vadose Zone Sediment from Borehole C3393.

4.1.6 Total Carbon, Calcium Carbonate, and Organic Carbon Content of Vadose Zone Sediment from Borehole C3393 ……......................................................... 4.13

4.1.7 Particle Size Measurements on Vadose Zone Sediment ........................................ 4.14

4.1.8 Matric Suction Potential Measurements.................................................................. 4.16

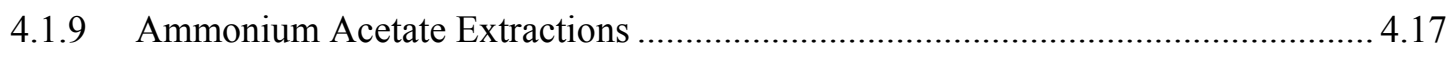

4.2 Vadose Zone Sediment from the U Tank Farm Direct Push Samples ............................... 4.18

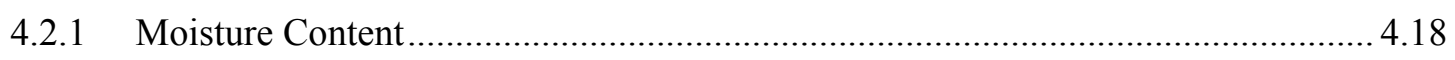

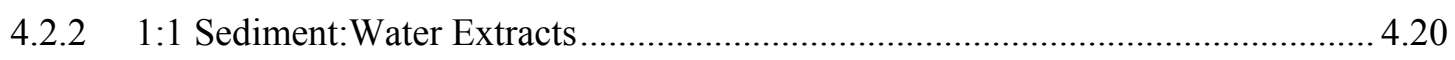

4.2.3 Vadose Zone Porewater Chemical Composition.................................................. 4.31

4.2.4 8 M Nitric Acid-Extractable Amounts of Selected Elements in the U Tank Farm Direct Push Sediments ......................................................................... 4.36

4.2.5 Radionuclide Content in Vadose Zone Sediment from the U Tank Farm Direct Push Holes Determined by GEA and Gross Alpha and Beta Analysis ........ 4.42

4.2.6 Total Carbon, Calcium Carbonate, and Organic Carbon Content of Vadose Zone Sediment from the U Tank Farm Direct Push Holes...................................... 4.45

4.2.7 Particle Size Measurements on U Farm Direct Push Vadose Zone Sediment ........ 4.46

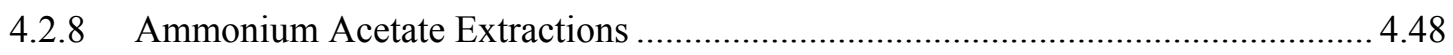

4.3 Detailed Characterization to Elucidate Controlling Geochemical Processes at the

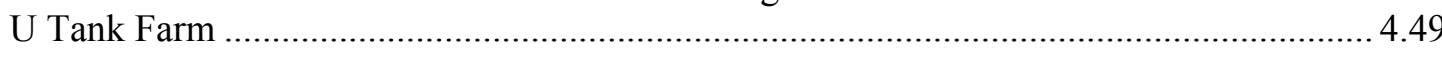

4.4 Estimates of Contaminant Partition Coefficients …........................................................ 4.50

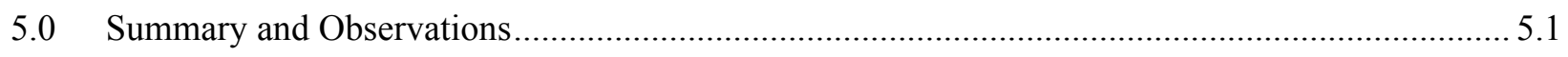

5.1 U Tank Farm Physical Geology Model...................................................................... 5.1

5.2 U Tank Farm Characterization Activities and Data ....................................................... 5.1

5.2.1 Sampling Summary at the U Tank Farm .......................................................... 5.1

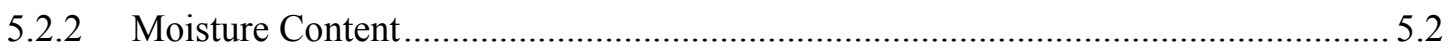

5.2.3 Contamination Profile around tanks 241-TY-105 and 241-TY-106 ......................... 5.2

5.2.4 Sources of Contamination in the U Tank Farm ....................................................... 5.4

5.3 Controlling Geochemical Processes at the U Tank Farm................................................. 5.5

5.4 Estimates of Contaminant Partition Coefficients ................................................................ 5.5

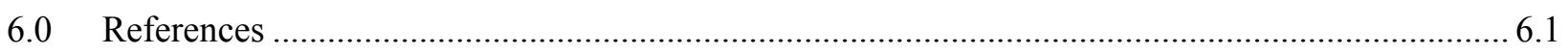

Appendix A - Photographs of Core and Grab Samples Opened in the Laboratory from Direct-Push Boreholes around Single-Shell Tanks in the 241-U Tank Farm.............................................. A.1

Appendix B - Core Logs of Core and Grab Samples Opened in the Laboratory from Direct-Push Boreholes around Single-Shell Tanks in the 241-U Tank Farm ................................................1

Appendix C - Photographs of Core Samples Opened in the Laboratory from Borehole C3393 to the East of the 241-U Tank Farm 


\section{Figures}

Figure 2.1. Fence Diagram Showing the Relationship Between Stratigraphic Units at Waste Management Area U .

Figure 2.2. Northwest-Southeast Geologic Cross Section of Waste Management Area U.

Figure 2.3. Outcrop Exposure of Gravel-Dominated (GD) Facies of the Hanford Formation

Figure 2.4. Outcrop Exposure of Sand-Dominated (SD) Facies of the Hanford Formation

Figure 2.5. Outcrop Exposure of Interbedded Sand- to Silt-Dominated (ISSD) Facies of the Hanford Formation

Figure 2.6. A Pair of Vertical Clastic Dikes

Figure 2.7. A Polygonal, Clastic Dike Network Exposed atop the Deflated Surface of the Hanford Formation

Figure 2.8. Detailed Hydrogeologic Cross Section (A - A') of the Vadose Zone Beneath U Tank Farm

Figure 3.1. Location Map of U Tank Farm.

Figure 4.1. Particle-Size Distribution of Sediment Sub-Samples from Borehole C3393

Figure 4.2. Matric Water Potential Measured by Filter Paper Technique on Core Samples from Borehole C3393

Figure 4.3. 1:1 Sediment:Water-Extractable Technetium-99 Data from Direct Push C5608 Samples

Figure 4.4. 1:1 Sediment:Water-Extractable Uranium-238 Data from Direct Push Hole C5602 Samples

Figure 4.5. Acid-Extractable Uranium-238 Data from Direct Push Hole C5602 Samples.

Figure 4.6. 1:1 Sediment:Water-Extractable Uranium-238 Data from Direct Push Hole C5602 Samples 


\section{Tables}

Table 2.1. Stratigraphic Terminology and Unit Thickness for the Vadose Zone Beneath the U

Tank Farm

Table 3.1. Sample Inventory from the U Tank Farm Background Borehole .......................................... 3.2

Table 3.2. Sample Inventory from the U Tank Farm Direct Push Holes ............................................... 3.3

Table 4.1. Gravimetric Moisture Content of Samples Obtained from the U Tank Farm Background Borehole

Table 4.2. $\mathrm{pH}$ for 1:1 Sediment:Water Extracts and Dilution-Corrected EC Values from Borehole C3393

Table 4.3. Water-Extractable Anions in the U Tank Farm Background Borehole

Table 4.4. Water-Extractable Major Cations in the U Farm Background Borehole.

Table 4.5. Water-Extractable Cations in the C3393 Borehole Core Samples.

Table 4.6. Water-Extractable Mobile Metals in the C3393 Borehole Core Samples

Table 4.7. Calculated Pore Water Anion Concentrations in the C3393 Borehole Core Samples............ 4.9

Table 4.8. Calculated Pore Water Cation Concentrations in the C3393 Borehole Core Samples

Table 4.9. Calculated Pore Water Mobile Metal Concentrations of Key Contaminants of Concern in the C3393 Borehole Samples

Table 4.10. Acid-Extractable Cations in the U Tank Farm Background Borehole Samples

Table 4.11. Acid-Leachable Cations in the U Tank Farm Background Borehole Samples

Table 4.12. Acid-Extractable Mobile Metals in the U Tank Farm Background Borehole Samples....... 4.12

Table 4.13. Total, Inorganic, and Organic Carbon Content of Vadose Zone Sediments from Borehole C3393.

Table 4.14. Wet Sieve Particle Size Results for Borehole C3393 Sediments

Table 4.15. Particle Size Data for Borehole C3393 Sediments Using Two Techniques Reported as Cumulative Percent Finer Than

Table 4.16. Matric Potential as Measured by Filter Paper Method for Borehole C3393 Core Sediments.

Table 4.17. Ammonium Acetate-Extractable Cations in the U Tank Farm Background Borehole Samples

Table 4.18. Cation Exchange Capacity (CEC) of Sediments from the Background Borehole...

Table 4.19. Gravimetric Moisture Content of Samples Obtained from the U Tank Farm Direct Push Holes

Table 4.20. $\mathrm{pH}$ for 1:1 Sediment:Water Extracts and Dilution-Corrected EC Values from U Tank Farm Samples

Table 4.21. Water-Extractable Anions in the U Tank Farm Core Samples

Table 4.22. Water-Extractable Major Cations in the U Tank Farm Direct Push Samples 
Table 4.23. Water-Extractable Cations in the U Tank Farm Core Samples

Table 4.24. Water-Extractable Mobile Metals in the U Tank Farm Core Samples .............................. 4.28

Table 4.25. Calculated Pore Water Anion Concentrations in the U Tank Farm Core Samples............. 4.31

Table 4.26. Calculated Pore Water Cation Concentrations in the U Tank Farm Direct Push Core Samples

Table 4.27. Calculated Pore Water Mobile Metal Concentrations of Key Contaminants of Concern in the U Tank Farm Direct Push Samples

Table 4.28. Acid-Extractable Cations in the U Tank Farm Direct Push Samples

Table 4.29. Acid-Leachable Cations in the U Tank Farm Core and Grab Samples

Table 4.30. Acid-Extractable Mobile Metals in the U Tank Farm Direct Push Samples

Table 4.31. Gamma-Emitting Radionuclides in the U Tank Farm Direct Push Sediments

Table 4.32. Gross Beta vs. ICP-MS Data in the U Tank Farm Direct Push Water Extracts.

Table 4.33. Gross Alpha and Beta Data in the U Tank Farm Direct Push Acid Extracts

Table 4.34. Total, Inorganic, and Organic Carbon Content of Vadose Zone Sediments from the Direct Push Holes

Table 4.35. Wet Sieve Particle Size Results for U Farm Direct Push Sediments

Table 4.36. Particle Size Data for U Farm Direct Push Sediments Using Two Techniques Reported as Cumulative Percent Finer Than

Table 4.37. Ammonium Acetate-Extractable Cations in the U Farm Direct Push Samples

Table 4.38. Cation Exchange Capacity (CEC) of Sediments from the U Farm Direct Push Holes

Table 4.39. Maximum Pore Water Concentrations in Sediments from the Hanford formation Unit ..... 4.50

Table 4.40. Equilibrium $K_{d}$ Values for the U Farm Direct Push Samples 4.51 


\section{Acronyms and Abbreviations}

\begin{tabular}{|c|c|}
\hline$\%$ RSD & percent relative standard deviation \\
\hline ASA & American Society of Agronomy \\
\hline ASTM & American Society for Testing and Materials \\
\hline bgs & below ground surface \\
\hline $\mathrm{CCU}$ & Cold Creek unit \\
\hline CEC & cation exchange capacity \\
\hline $\mathrm{CMB}$ & chloride mass balance \\
\hline DOE & U.S. Department of Energy \\
\hline $\mathrm{EC}$ & electrical conductivity \\
\hline EPA & U.S. Environmental Protection Agency \\
\hline EQL & estimated quantification limit \\
\hline GD & gravel dominated \\
\hline GEA & gamma energy analysis \\
\hline H1 & Hanford formation - H1 unit \\
\hline $\mathrm{H} 2$ & Hanford formation $-\mathrm{H} 2$ unit \\
\hline $\mathrm{HCl}$ & hydrochloric acid \\
\hline HEIS & Hanford Environmental Information System \\
\hline $\mathrm{HF}$ & hydrofluoric acid \\
\hline $\mathrm{HNO}_{3}$ & nitric acid \\
\hline $\mathrm{H} / \mathrm{PP}$ & Hanford formation/PlioPleistocene \\
\hline HPGe & high-purity germanium \\
\hline HRR & high-resolution resistivity \\
\hline IC & ion chromatography or ion chromatograph \\
\hline ICP-MS & inductively coupled plasma-mass spectrometer \\
\hline ICP-OES & inductively coupled plasma-optical emission spectroscopy \\
\hline ILAW & Integrated Low Activity Waste \\
\hline ISSD & interbedded sand-to silt-dominated \\
\hline $\mathrm{Kd}$ & distribution coefficient, or sorption partition coefficient, in units of $\mathrm{mL} / \mathrm{g}$ \\
\hline $\mathrm{MC}$ & moisture content \\
\hline MCL & maximum contamination level \\
\hline $\mathrm{mS}$ & millisiemen \\
\hline NDIR & non-dispersive infrared \\
\hline NIST & National Institute of Standards and Technology \\
\hline PNNL & Pacific Northwest National Laboratory \\
\hline QA & quality assurance \\
\hline QC & quality control \\
\hline RCRA & Resource Conservation and Recovery Act of 1976 \\
\hline REDOX & $\begin{array}{l}\text { Reduction Oxidation Process (the second fuel reprocessing process used at the Hanford } \\
\text { Site to extract plutonium) }\end{array}$ \\
\hline
\end{tabular}




$\begin{array}{ll}\text { Rtf } & \text { Ringold Formation sand } \\ \text { Rwi } & \text { Ringold fluvial gravel } \\ \text { SD } & \text { sand dominated } \\ \text { TBP } & \text { Tributyl phosphate } \\ \text { UFA } & \text { unsaturated flow apparatus (ultracentrifuge for squeezing pore water out of sediment) } \\ \text { UPR } & \text { unplanned release } \\ \text { USGS } & \text { U.S. Geological Survey } \\ \text { WMA } & \text { waste management area }\end{array}$

\section{Units of Measure}

$\begin{array}{ll}\% & \text { percent } \\ \mathrm{Bgs} & \text { below ground surface } \\ { }^{\circ} \mathrm{C} & \left.\text { temperature in degrees Celsius }\left[\mathrm{T}\left({ }^{\circ} \mathrm{C}\right)=\mathrm{T}(\mathrm{K})-273.15\right)\right] \\ \mathrm{Ci} & \text { curie } \\ \mathrm{cm} & \text { centimeter } \\ \mathrm{ft} & \text { foot } \\ \mathrm{g} & \text { gram } \\ \mathrm{g} & \text { acceleration due to gravity } \\ \mathrm{in} . & \text { inch } \\ \mu & \text { micro (prefix, } 10-6) \\ \mu \mathrm{Ci} & \text { microcurie } \\ \mu \mathrm{eq} & \text { microequivalent } \\ \mu \mathrm{g} & \text { microgram } \\ \mu \mathrm{m} & \text { micrometer } \\ \mathrm{m} & \text { meter } \\ \mathrm{M} & \text { molarity, mol/L } \\ \mathrm{meq} / \mathrm{L} & \text { milli-equivalent per liter } \\ \mathrm{mg} & \text { milligram } \\ \mathrm{mL} & \text { milliliter } \\ \mathrm{mm} & \text { millimeter } \\ \mathrm{mM} & \text { millimolar } \\ \mathrm{mN} & \text { millinormal } \\ \mathrm{mol} & \text { mole } \\ \mathrm{MPa} & \text { Megapascal } \\ \mathrm{mS} & \text { milliSiemen } \\ \mathrm{N} & \text { Normal } \\ \mathrm{nCi} & \text { nanocurie } \\ \mathrm{ng} & \text { nanogram } \\ \mathrm{pCi} & \text { picocurie } \\ \mathrm{wt} \% & \text { weight percent } \\ & \end{array}$




\subsection{Introduction}

In order to understand the extent and degree of contamination in the single-shell Waste Management Areas (WMAs), detailed geochemical and mineralogical studies are needed. These efforts are aimed at better elucidating the migration and retention behavior of contaminant molecules that interact with sediments after they were released into the environment. If the various sinks and transit pathways in the sediments can be better understood, then there is a prospect that enlightened environmental remediation methods can be established. Many of these goals are laid out in the work plan fashioned for the Tank Farm Vadose Zone Project, which has been entitled: Phase 1 RCRA Facility Investigation/Corrective Measures Study Work Plan for the Single-Shell Tank Waste Management Areas (DOE 1999). Because each WMA has experienced different degrees of contamination with a variety of different pollutants, the remedial strategy for each is different. In the case of the $U$ tank farm, or WMA U, several tanks have been suspected of leaking and results from remote sensing methods (high resolution resistivity) appear to corroborate this suspicion. On the other hand, spectral gamma logging in certain drywells has not revealed evidence of contamination, as discussed below. These and other data gaps motivated Crumpler (2003) to define a list of characterization objectives for WMA U. To meet these goals, CH2M HILL Hanford Group, Inc., petitioned scientists from Pacific Northwest National Laboratory (PNNL) to perform detailed analyses of vadose zone sediment collected from boreholes emplaced within the U Single-Shell Tank Farm.

This report contains all the geochemical and selected physical characterization data collected on vadose zone sediment recovered from ten direct push characterization holes emplaced to investigate vadose zone contamination associated with potential leaks within the 241-U Single-Shell Tank Farm. Specific tanks targeted during this characterization campaign included tanks 241-U-104/241-U-105, 241U-110, and 241-U-112. Tank 241-U-104 is estimated to have leaked 190,000 L (50,000 gallons) of bismuth phosphate metals waste to the vadose zone prior to 1956 (Crumpler 2003). Recent high resolution resistivity (HRR) data (Rucker et al. 2006) indicated that the majority of the dissolved salts contained in the waste plume associated with the 241-U-104 leak event may reside under tank 241-U-105, which was the primary motivation for the combined 241-U-104/241-U-105 characterization campaign.

Tanks 241-U-110 and 241-U-112 are both estimated to have leaked small amounts of waste to the vadose zone. Cesium-137 measured in drywells 60-10-07 (located southwest of tank 241-U-110) and 6012-01 (located north of tank 241-U-112) indicates that the leaks likely occurred at the bottom of the tanks. Both tanks were used to store high-temperature wastes, and REDOX supernatant was the primary waste stored in both of the tanks. Two direct push holes were emplaced adjacent to each tank; one direct push hole was used to collect geophysical data while the other hole was used to retrieve vadose zone sediment samples for subsequent characterization. The direct push holes were emplaced near drywells 60-12-01 and 60-10-07 to look for additional tank waste constituents in the vadose zone and specifically mobile contaminants that are undetectable via spectral gamma logging.

Tank 241-U-101 is the only other U Farm single-shell tank suspected of having leaked. Tank 241-U101 is reported to have leaked $114,000 \mathrm{~L}$ (30,000 gallons) of high-level waste in 1959. Spectral gamma logging of drywells located to the east, south, and west of tank 241-U-101 does not provide evidence of a leak from the tank. However, no drywells exist to the north/northeast of tank 241-U-101, which leaves a large data gap in assessing the integrity of the tank. Therefore, two sets of direct push holes were 
emplaced north and northeast of tank 241-U-101 to provide geophysical and sediment characterization data.

The final area investigated as part of the U Farm direct push campaign was just north of tank 241-U201. The recent HRR data acquired by Rucker et al. (2006) indicated the presence of a resistivity anomaly near the U farm 200 series tanks. Although no leaks are known to have occurred in the area of the 200 series tanks, two direct push holes were emplaced to directly investigate the resistivity anomaly.

Finally, this report contains all the geochemical and selected physical characterization data collected on vadose zone sediment recovered from a Resource Conservation and Recovery Act (RCRA) groundwater monitoring well emplaced east of the 241-U tank farm.

This report is divided into sections that describe the geochemical characterization methods employed and the results of analysis of the vadose zone samples. English units are used in this report for descriptions and discussions of drilling activities and samples because that is the system of units used by drillers to measure and report depths. To convert feet to meters, multiply by 0.3048 ; to convert inches to centimeters, multiply by 2.54 . The metric system is used in this report for all other purposes. 


\subsection{Geology}

The geology in the vicinity of $U$ tank Farm has been discussed in a number of previous reports (Price and Fecht 1976, Hodges and Chou 2000, Smith et al. 2001, Wood and Jones 2003, and Reidel and Chamness 2007). The generalized stratigraphy beneath the Hanford Site and U Tank Farm consists of, in ascending order, the Columbia River Basalt Group, the Ringold Formation, the Cold Creek Unit (CCU) (formerly named the Plio-Pleistocene unit), and the Hanford formation (Table 2.1). The CCU and Hanford formation are both informal designations. A fence diagram illustrating the variations in lithologic thickness within the 200 Areas is presented in Figure 2.1. The Cold Creek unit and Ringold Formation both tilt gently to the southwest $\left(<1^{\circ}\right)$ beneath $U$ Tank Farm. The total thickness of suprabasalt sediment is about $170 \mathrm{~m}(560 \mathrm{ft})$ beneath $U$ Tank Farm. The lateral and vertical distributions of the different units are represented in Figures 2.1 and 2.2, showing that the dimensions of the main units are generally homogeneous in thickness and distribution in this area.

\subsection{Stratigraphy of the Vadose Zone Beneath the U Tank Farm}

\subsubsection{Ringold Formation}

The lower portion of the vadose zone lies within fluvial, braided stream deposits of the Ringold Formation. These deposits belong to Unit E member of Wooded Island (Lindsey 1995). Because no direct-push samples were collected for geochemical analysis from the Ringold Formation, these strata are not discussed in any detail in this report. For more information on both the saturated and unsaturated portions of the Ringold Formation we suggest the following documents: DOE 1988; Lindsey 1992, 1995; Reidel and Chamness 2007.

\subsubsection{Cold Creek unit (CCU)}

The CCU unconformably overlies the Ringold Formation (Wood and Jones 2003) and basalt-rich gravel and is divided into an upper $\left(\mathrm{CCU}_{1}\right)$ and lower $\left(\mathrm{CCU}_{\mathrm{u}}\right)$ subunit. The $\mathrm{CCU}_{1}$ is a caliche-rich zone about 5 to $10 \mathrm{ft}$ thick that developed on the paleo-surface of the Ringold Formation. The caliche layer is superimposed on the eroded and weathered surface of the Ringold Formation, in Unit E in the vicinity of the U Tank Farm. The upper subunit $\left(\mathrm{CCU}_{\mathrm{u}}\right)$ is a well sorted and relatively un-weathered, brown-colored silt-rich deposit. Both subunits have a slight regional dip to the southwest. The upper fine-grained and lower caliche-cemented portions of the Cold Creek unit strongly impede the vertical movement of pore fluids in the vadose zone.

The CCU represents deposits that accumulated within the central Pasco Basin during the period between about 2 and 3 million years ago, which brackets two significant geologic events. The older event is a regional base-level drop and subsequent incision of the Ringold Formation (DOE 1988). The younger event is the initiation of Ice Age cataclysmic flooding, which began at the beginning of the Pleistocene, about 1.5 to 2.5 million years ago (Bjornstad et al. 2001; Bjornstad 2006).

Because no direct-push samples were collected for geochemical analysis from the Cold Creek unit, it will not be discussed in any detail in this report. For more information on the CCU we suggest the following documents: Slate 1996, 2000; DOE 2002; Reidel and Chamness 2007. 
Table 2.1. Stratigraphic Terminology and Unit Thickness for the Vadose Zone Beneath the U Tank Farm. Modified after DOE (2002).

\begin{tabular}{|c|c|c|c|c|c|}
\hline $\begin{array}{c}\text { Stratigraphic } \\
\text { Symbol }\end{array}$ & Formation & $\begin{array}{l}\text { Facies/ } \\
\text { Subunit }\end{array}$ & Description & Origin & $\begin{array}{c}\text { Thickness } \\
\text { (ft) }\end{array}$ \\
\hline Backfill & NA & Backfill & $\begin{array}{l}\text { Gravel-dominated consisting of poorly to moderately } \\
\text { sorted cobbles, pebbles, and coarse to medium sand } \\
\text { with some silt derived from coarse-grained Hanford } \\
\text { formation (H1 unit) excavated around tanks (Price and } \\
\text { Fecht 1976; Wood et al. 2001); sparsely distributed } \\
\text { layers of sand to silty sand occur near the base of the } \\
\text { backfill sequence. }\end{array}$ & Anthropogenic & 36 \\
\hline H1 & \multirow{2}{*}{$\begin{array}{l}\text { Hanford } \\
\text { formation }\end{array}$} & $\begin{array}{l}\text { Unit H1 - } \\
\text { (Gravel- } \\
\text { dominated } \\
\text { facies } \\
\text { association). }\end{array}$ & $\begin{array}{l}\text { Gravel-dominated flood sequence; composed of } \\
\text { mostly poorly sorted, basaltic, sandy gravel to silty } \\
\text { sandy gravel. Equivalent to the upper gravel sequence } \\
\text { discussed by Last et al. (1989), the } \mathrm{Q}_{\mathrm{fg}} \text { (or Quaternary } \\
\text { flood, gravel-dominated) documented by Reidel and } \\
\text { Fecht (1994b), Hanford Gravel Unit A of Johnson et } \\
\text { al. (1999), coarse-grained sequence (H1 unit) of Wood } \\
\text { et al. (2001) and gravel facies of unit H1 of Lindsey } \\
\text { et al. (2001b), and gravel-dominated facies association } \\
\text { of DOE-RL (2002). }\end{array}$ & $\begin{array}{l}\text { Cataclysmic } \\
\text { outburst } \\
\text { floods (high } \\
\text { energy) }\end{array}$ & $10-70$ \\
\hline $\mathrm{H} 2$ & & \begin{tabular}{|l} 
Unit $\mathrm{H} 2-$ \\
(Sand- \\
dominated \\
facies \\
association).
\end{tabular} & $\begin{array}{l}\text { Sand-dominated flood sequence; composed of } \\
\text { mostly horizontal to tabular cross-bedded sand to } \\
\text { gravelly sand. Many sand beds capped with thin } \\
\text { layers of silty sand to sandy silt. Equivalent to } \\
\text { Hanford Sands of Johnson et al. (1999), Fine-Grained } \\
\text { Sequence (H2 unit) of Wood et al. (2001), and unit H2 } \\
\text { of Lindsey et al. (2001a), the sandy sequence of Last } \\
\text { et al. (1989), and to } \mathrm{Q}_{\mathrm{fs}} \text { (or Quaternary flood, sand- } \\
\text { dominated) documented by Reidel and Fecht (1994b) } \\
\text { and sand-dominated facies association of DOE-RL } \\
(2002) \text {. }\end{array}$ & $\begin{array}{l}\text { Cataclysmic } \\
\text { outburst } \\
\text { floods } \\
\text { (moderate to } \\
\text { low energy) }\end{array}$ & $50-75$ \\
\hline $\mathrm{CCU}_{\mathrm{u}}$ & \multirow{2}{*}{$\begin{array}{c}\text { Cold } \\
\text { Creek unit }\end{array}$} & $\begin{array}{l}\text { Upper } \\
\text { subunit }\end{array}$ & $\begin{array}{l}\text { Silty sequence consisting of massive to interstratified, } \\
\text { well sorted silt and fine sand. Uncemented but may } \\
\text { be moderately to strongly calcareous from detrital } \\
\text { CaCO } \text {. Equivalent to the "early Palouse soil" (Brown }_{19} \text { 1960; Tallman et al. 1979; DOE 1988; and DOE-GJO } \\
\text { 1997) and the Hanford Formation(?)/Plio-Pleistocene } \\
\text { (?) deposits (H/PP) of Wood et al. (2001). Also } \\
\text { equivalent to the upper Plio-Pleistocene unit (Lindsey } \\
\text { et al. } 2001 \text { a; Sobczyk 2000) and the fine-grained, } \\
\text { laminated to massive [CCUf(lam-msv)] lithofacies of } \\
\text { the Cold Creek unit (DOE 2002). Same as PPu of } \\
\text { Lindsey et al. (2001b). }\end{array}$ & $\begin{array}{l}\text { Post-Ringold } \\
\text { Fm. eolian } \\
\text { and/or } \\
\text { overbank } \\
\text { alluvial } \\
\text { deposits }\end{array}$ & $10-15$ \\
\hline $\mathrm{CCU}_{1}$ & & $\begin{array}{l}\text { Lower } \\
\text { subunit }\end{array}$ & $\begin{array}{l}\text { Calcic paleosol sequence, consisting of interbedded } \\
\text { layers of pedogenically altered or unaltered gravel, } \\
\text { sand, silt, and/or clay, cemented together with one or } \\
\text { more layers of secondary } \mathrm{CaCO}_{3} \text {, originally referred } \\
\text { to as "caliche" (Brown 1959). Since then the name } \\
\text { has evolved from the Plio-Pleistocene unit (Bjornstad } \\
\text { 1984, 1990; DOE 1988; DOE-GJO 1997; Slate 2000), } \\
\text { the Plio-Pleistocene calcrete facies (DOE 1988; Wood } \\
\text { et al. 2001), the lower Plio-Pleistocene unit (Lindsey } \\
\text { et al. (2001b), and the coarse- to fine-grained, } \mathrm{CaCO}_{3^{-}} \\
\text {cemented lithofacies [CCUc-f(calc)] of the Cold }\end{array}$ & \begin{tabular}{|l|} 
Calcic \\
paleosols \\
developed on \\
top of eroded \\
and weathered \\
Ringold Fm. \\
(Unit E)
\end{tabular} & $5-10$ \\
\hline
\end{tabular}




\begin{tabular}{|c|c|c|c|c|c|}
\hline $\begin{array}{c}\text { Stratigraphic } \\
\text { Symbol }\end{array}$ & Formation & $\begin{array}{l}\text { Facies/ } \\
\text { Subunit }\end{array}$ & Description & Origin & $\begin{array}{c}\text { Thickness } \\
\text { (ft) }\end{array}$ \\
\hline & & & $\begin{array}{l}\text { Creek unit (DOE 2002). Same as PPc of Lindsey et } \\
\text { al. (2001b). }\end{array}$ & & \\
\hline $\mathrm{R}_{\mathrm{wi}}$ & $\begin{array}{l}\text { Ringold } \\
\text { Formation }\end{array}$ & $\begin{array}{l}\text { Member of } \\
\text { Wooded } \\
\text { Island (Unit } \\
\text { E) }\end{array}$ & $\begin{array}{l}\text { Coarse-grained Ringold Formation sequence, } \\
\text { consisting of mostly moderately sorted, quartzitic } \\
\text { sandy gravel to silty sandy gravel. Equivalent to } \\
\text { middle Ringold Formation unit (DOE 1988) and the } \\
\text { Ringold Formation unit E gravels (Wood et al. 2001; } \\
\text { Lindsey et al. 2001b). Well-stratified clay and } \\
\text { interbedded silt and silty sand is equivalent to the } \\
\text { lower mud Ringold Formation unit (DOE 1988). } \\
\text { Fluvial gravels with intercalated sands are equivalent } \\
\text { to the basal Ringold Formation unit (DOE 1988) and } \\
\text { the Ringold Formation unit A gravels (Wood et al. } \\
\text { 2001; Lindsey et al. 2001b). }\end{array}$ & \begin{tabular}{|l|} 
Ancestral \\
Columbia \\
River system \\
braided-stream \\
deposits
\end{tabular} & $\sim 300$ \\
\hline
\end{tabular}

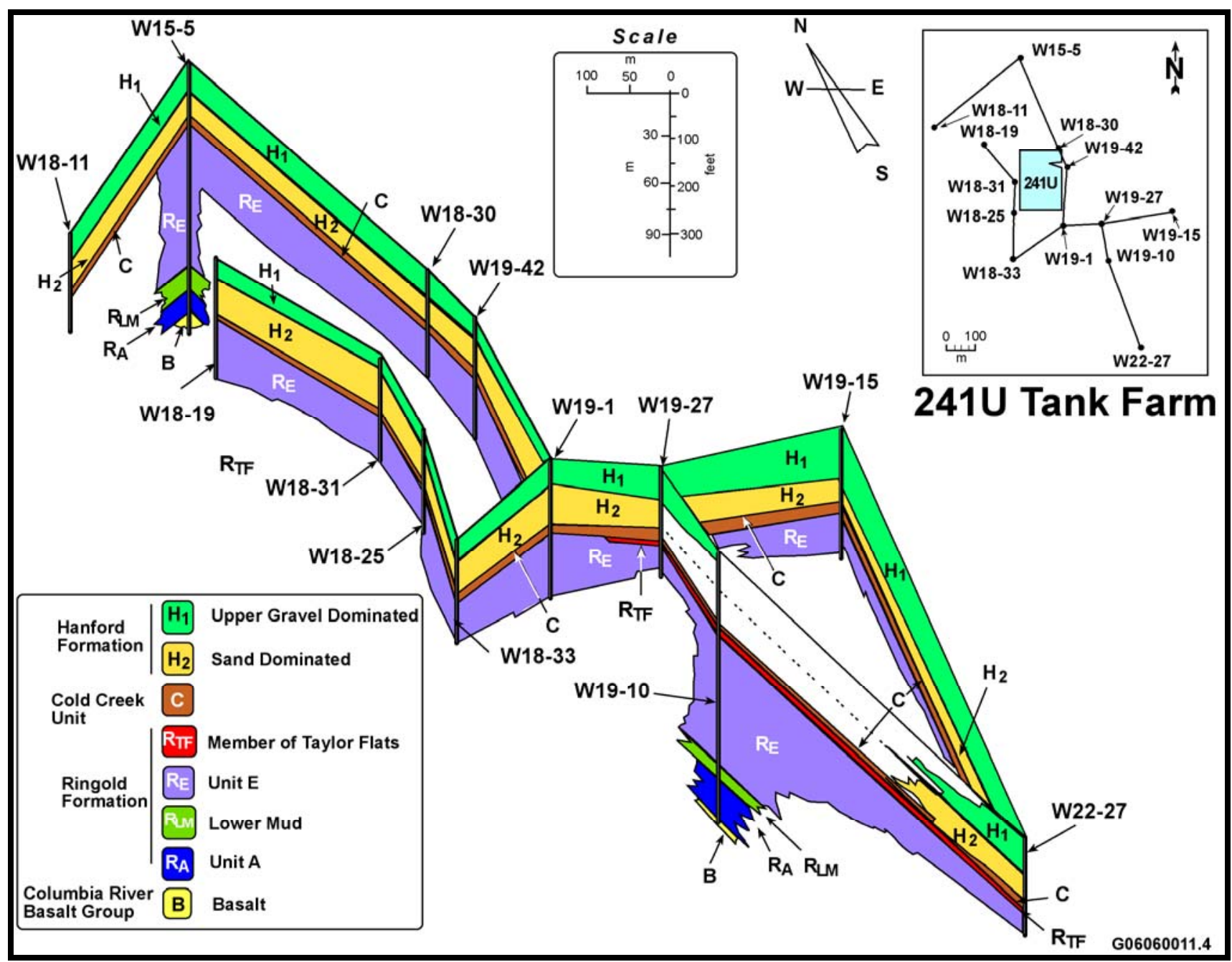

Figure 2.1. Fence Diagram Showing the Relationship Between Stratigraphic Units at Waste Management Area U (after Reidel and Chamness 2007) 


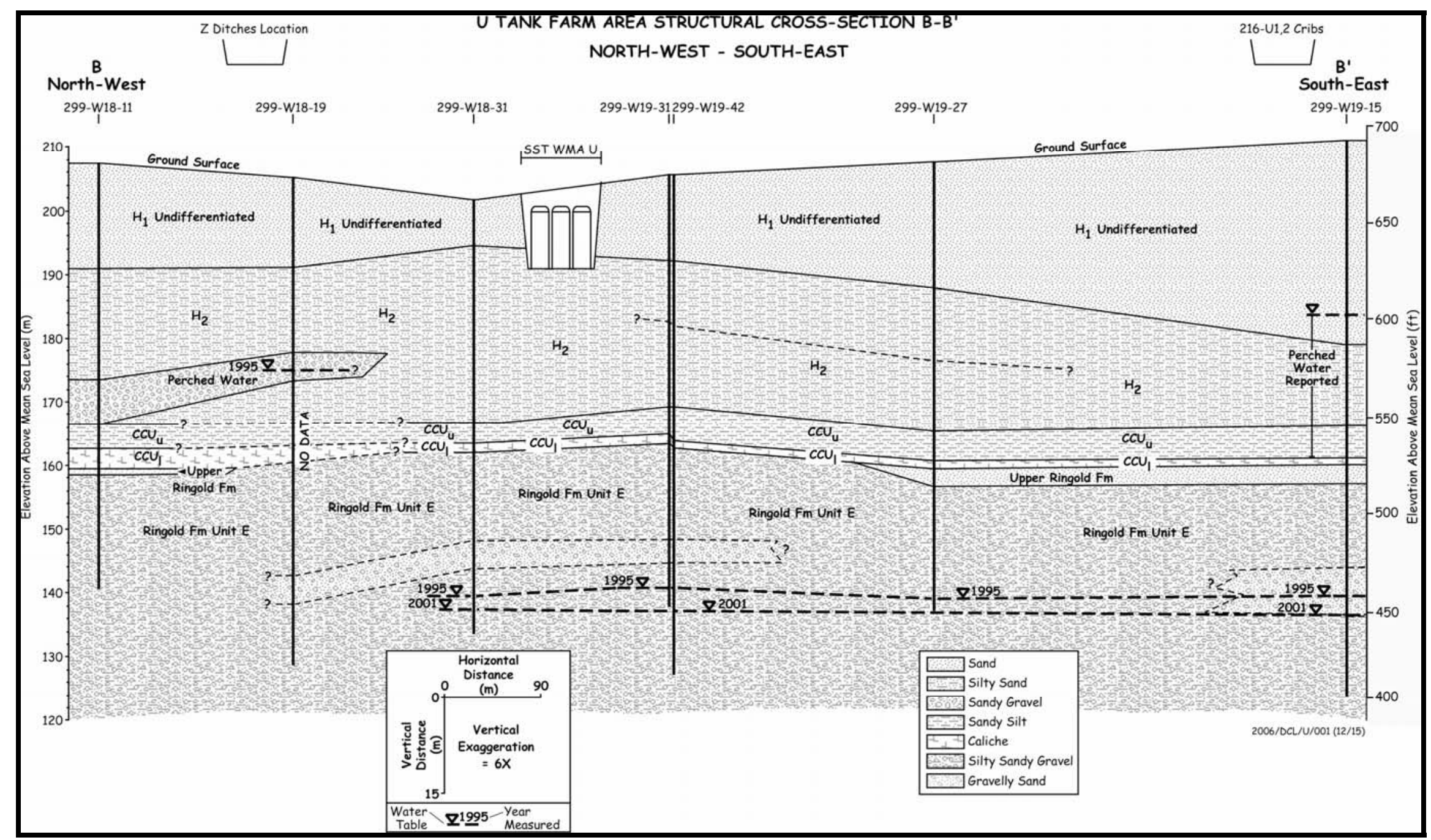

Figure 2.2. Northwest-Southeast Geologic Cross Section of Waste Management Area U (after Smith et al. 2001) 


\subsubsection{Hanford formation}

The Hanford formation is composed of sediments deposited during several episodes of cataclysmic flooding and consists consists of poorly-sorted sand containing lithic fragments from pebble to boulder size, fine- to coarse-grained sand, and silt (DOE 1988, 2002). The Hanford formation is divided into two major sequences based on lithology at U Tank Farm - lower sand- (with interbedded silt) dominated (H2) and upper gravel-dominated (H1) sequences (Smith et al. 2001; Wood and Jones 2003). The coarsegrained (H1) sequence is distinguished from the finer-grained $\mathrm{H} 2$ sequence by a marked difference in grain-size distribution. A significant fraction of the upper unit is gravels with less sand, indicating deposition in a higher-energy environment. In the vicinity of U Tank Farm, the contact between the two units appears to dip to the south and west.

The Hanford formation is an informal name assigned to Pleistocene cataclysmic flood deposits within the Pasco Basin (Tallman et al. 1979; DOE 1988, 2002). Ice-Age floods originated from periodic outbursts from glacial Lake Missoula and other Pleistocene water bodies (Bjornstad 2006). The Hanford formation may include some minor fluvial, colluvial, and/or eolian deposits interbedded with flood deposits.

The Hanford formation consists predominantly of unconsolidated sediments that cover a wide range in grain size and sorting, from poorly sorted boulder-bearing to moderately-sorted sand, silty sand, and silt. In general, the Hanford formation is subdivided into three principal facies: 1) gravel-dominated (GD), 2) sand-dominated (SD), and 3) interbedded sand- and silt-dominated (ISSD) [DOE 2002]. GD flood deposits formed toward the center of the basin where currents and energy were the strongest. In this vicinity smaller particles were kept in suspension by the fast moving, highly turbulent flood waters. As flood energy decreased southward toward the margins of the basin, flood deposits transitioned to the SD and ISSD facies. Because of the widely different and complex flow dynamics during Ice Age flooding, Hanford formation strata are heterogeneous and anisotropic (DOE 2002; Bjornstad 2006). The bulk of the vadose zone within the Pasco Basin and the Hanford Site lies within sediments of the Hanford formation.

During Ice Age flooding, sediments accumulated onto the huge Cold Creek Bar, which makes up the 200 Area Plateau, which includes the U Tank Farm. The Cold Creek Bar grew as sediments were episodically laid down in a series of perhaps hundreds of floods spanning a million years or more (Pluhar et al. 2006). A network of braided flood channels sweeping across the bar locally scoured into the preexisting deposits and were backfilled with coarse sand and gravel. Elsewhere, blankets of sand were laid down at higher elevations within and between these channels. The Cold Creek Bar is a major flood landform stretching up to 12 miles long and several miles wide, that grew during repeated Ice Age floods at the east end of Umtanum Ridge as flood waters expanded into the basin and dropped their sedimentary load.

GD flood facies of the Hanford formation are more prominant in the northern 200 West Area, which were closer to high-energy flood channels. These coarse-grained deposits transition laterally into finer grained deposits of sand and, eventually, sand interbedded with silt to the south (Figures 2.2 and 2.3). Flood deposits reached their maximum thickness $(300 \mathrm{ft})$ beneath Cold Creek Bar near the southwest corner of the 200 East Area. Flood deposits in the 200 West Area are much thinner $(\sim 25 \mathrm{ft})$ to the north where they overlie the rising $\mathrm{CCU}$, a hard, weathered surface that resisted erosion during Ice Age floods. 
In contrast, flood deposits of the Hanford formation are much thicker (up to $150 \mathrm{ft}$ ) in the southern 200 West Area due to the lower elevation of the CCU.

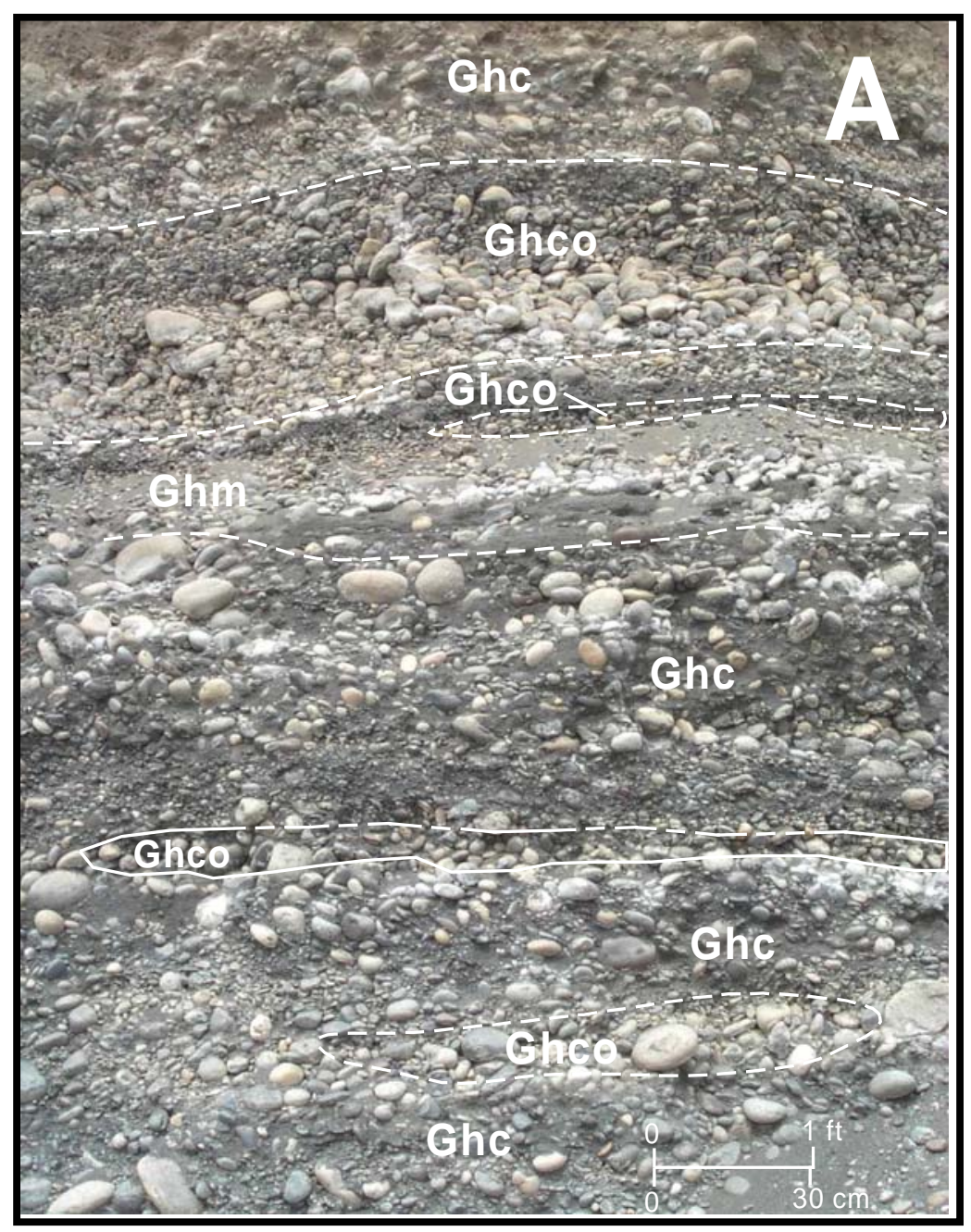

Figure 2.3. Outcrop Exposure of Gravel-Dominated (GD) Facies of the Hanford Formation. Lithofacies symbols: $\mathrm{Gh}=$ horizontally bedded gravel; Ghc = clast-supported, $\mathrm{Ghm}=$ matrix supported, Ghco= open work and clast supported.

Unlike the underlying Ringold Formation and CCU, the stratigraphy of the Hanford formation is much more complex and difficult to interpret. This is primarily due to the wide range and diversity of erosion and deposition events that occurred during the waxing and waning of perhaps hundreds of separate Ice Age flood events. The end result is a series of heterogeneous and laterally discontinuous strata within the Hanford formation. This was a much more complex depositional environment than that of the "simple" fluvial, pedogenic, and eolian environments that deposited the Ringold Formation and CCU strata. 


\subsubsection{Gravel-Dominated Facies Association}

Gravel-dominated (GD) facies were deposited by high-energy floodwaters in or immediately adjacent to the main cataclysmic flood channels. GD facies generally consist of poorly sorted, coarse-grained sand and pebble- to boulder-sized lithic fragments, which may display an open-framework fabric, massive bedding, plane to low-angle bedding, and/or large-scale, planar-tabular, fore-set bedding in outcrops (Figure 2.3). The gravel clasts (dominated by basalt) are usually subangular to subrounded. GD facies may grade vertically, as well as laterally, into SD facies.

Erosional unconformities (defined by intercalated finer-grained layers) may represent separate floods, but where fines have been completely eroded during subsequent floods, it is not possible to evaluate the number of floods. Therefore, these coarse grained deposits are typically lumped together leaving the appearance of a single flood event within most sequences of GD facies. In general, the absence of paleosols or other disconformities within flood-gravel sequences suggests that most or all older flood deposits were stripped away or reworked via erosional scouring by the last (late Pleistocene) flood(s). Occasionally, scour and fill features and gradations within flood-gravel sequences may be present, indicating multiple floods or flow variations that occurred during a single flood.

\subsubsection{Sand-Dominated (SD) Facies Association}

SD deposits of the Hanford formation consist of variably graded sand sequences several or more meters thick. Minor amounts of silty fine sand to silt may cap some of the beds. Generally, SD facies formed at higher elevations where floodwaters were starved of gravel, and/or adjacent to main flood channel ways during the dissipating stages of flooding, or perhaps as crevasse splay-like deposits proximal to overflowing flood channels. SD deposits of the Hanford formation typically display horizontal to ripple laminations in outcrops (Figure 2.4). Normal and reverse grading between different sand sizes is common, adding to the heterogeneity and anisotropy of this facies. Volumetrically, the SD facies is the predominant one within the 200 Areas. SD facies may grade vertically, as well as laterally, into GD facies or the ISSD facies.

\subsubsection{Inter-bedded Sand- to Silt- Dominated (ISSD) Facies Association}

ISSD facies characteristically consist of regularly graded beds of slackwater sand and silt, otherwise known as Touchet Beds or "rhythmites" (Figure 2.5). Individual rhythmites range from a few centimeters to a meter in thickness (Baker et al. 1991; Smith 1993), and when exposed in outcrops, can be traced laterally for hundreds of meters or more. Slackwater flood deposits of the ISSD facies are most prevalent around the margins of the Pasco Basin and up back-flooded tributary valleys along floods routes. Occasional pebble- to boulder-sized clasts found encompassed within the fine-grained matrix of facies association ISSD most likely represent ice-rafted erratics that floated in on icebergs (Bjornstad 2006).

ISSD facies of the Hanford formation provide a record of the occurrence of multiple floods. This is because in slackwater environments, the erosive power of the floods was diminished, resulting in little or no erosion during (and between) flood events. While minor erosion associated with localized scouring along the bases of some beds may occur, the upper portions of rhythmites are predominantly composed of silt that settled out of suspension and blanketed slackwater areas with a relatively continuous layer of cohesive, fine-grained sand and/or silt. This cohesive layer, which mantles most ISSD rhythmites, helps 
to protect the underlying rhythmites sequence from erosion during subsequent floods. ISSD facies may grade vertically, as well as laterally, into SD facies.

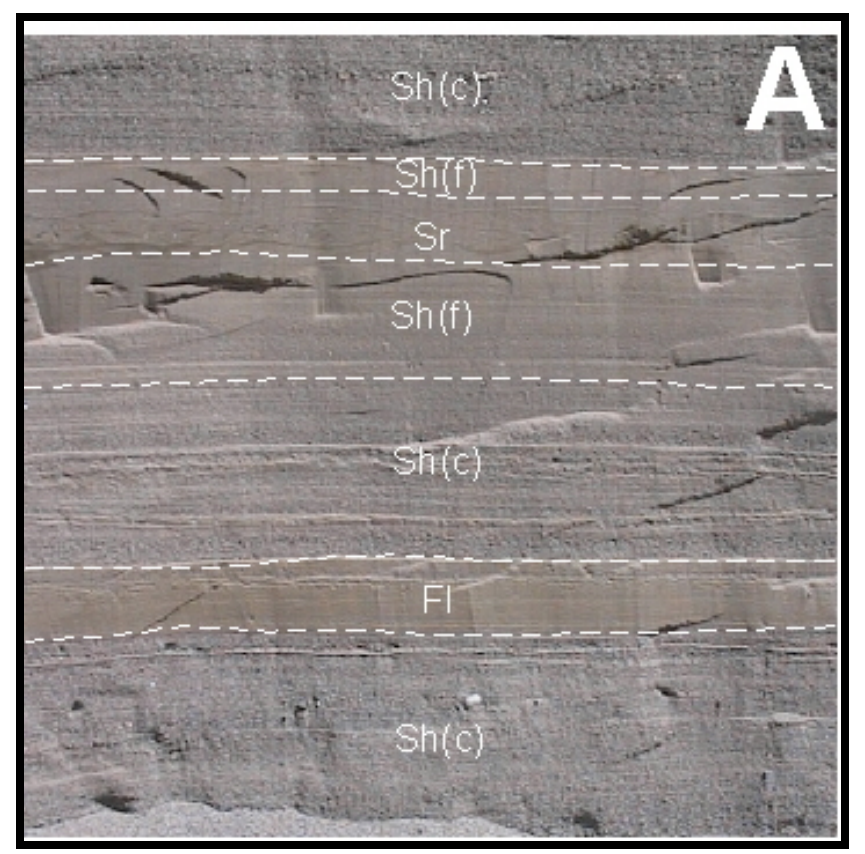

Figure 2.4. Outcrop Exposure of Sand-Dominated (SD) Facies of the Hanford Formation. Lithofacies symbols: $\operatorname{Sh}(\mathrm{f})=$ horizontally laminated fine sand, $\mathrm{Sh}(\mathrm{c})=$ horizontally laminated medium to coarse sand, $\mathrm{Sr}=$ ripple-laminated sand, $\mathrm{Fl}=$ laminated fine sand to silt.

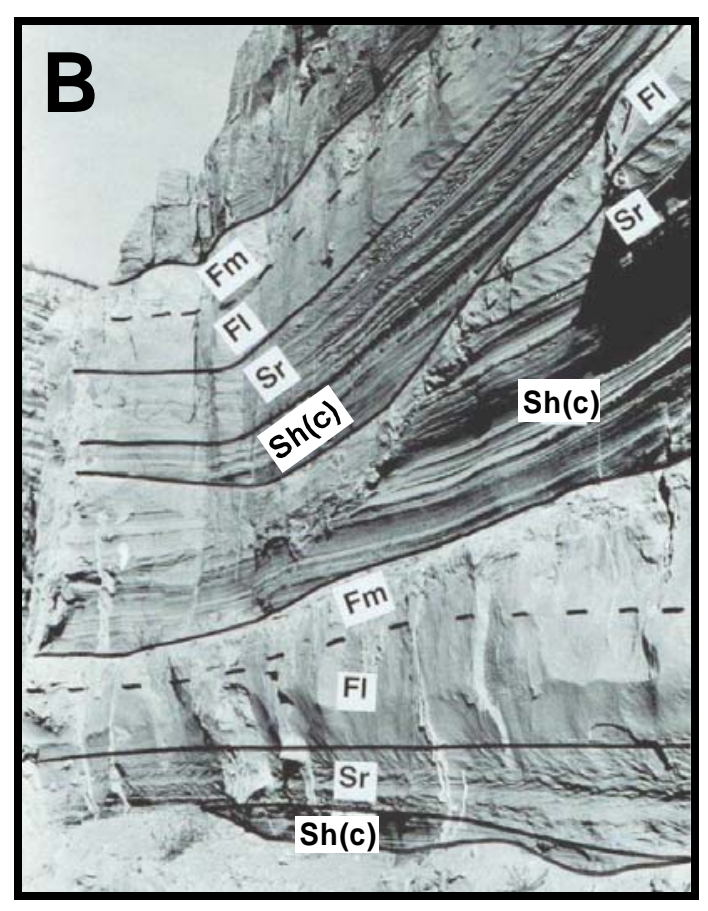

Figure 2.5. Outcrop Exposure of Interbedded Sand- to Silt-Dominated (ISSD) Facies of the Hanford Formation 


\subsubsection{Clastic Dikes}

A common feature of the SD and ISSD facies are subvertical clastic dikes. These discordant features cut across horizontally layered beds (Figure 2.6). Dikes vary from less than 1 inch to 6 feet or more wide and range from a few to over 100 feet long. They are commonly associated with, but not restricted to, slackwater flood deposits and most often are observed within the SD and ISSD facies of the Hanford formation. In contrast, clastic dikes are much less common in the GD facies (Bjornstad 2006).

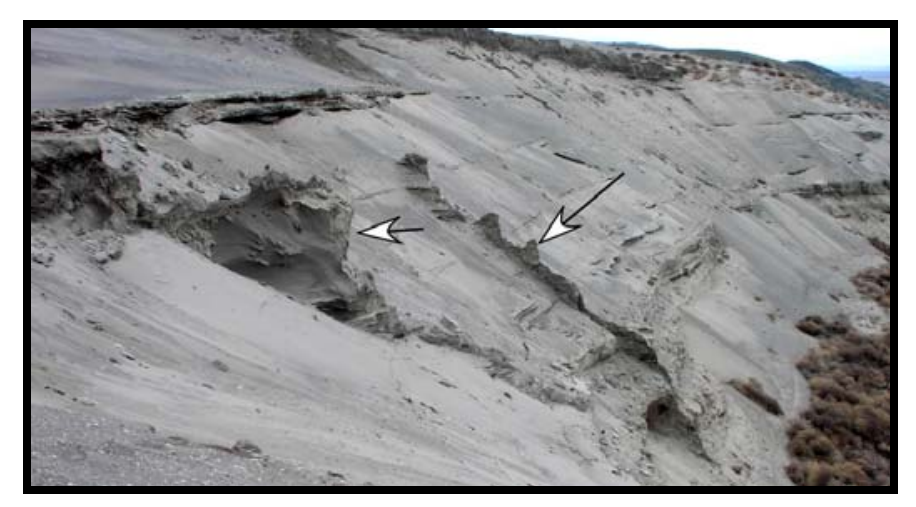

Figure 2.6. A Pair of Vertical Clastic Dikes (arrows). These dikes crosscut SD flood deposits exposed along the White Bluffs just east of the Hanford Site. The dikes stand out here because they contain more cohesive silt and are better able to withstand attack by strong winds, which are actively eroding these bluffs.

Most dikes occur as sharp-walled, near-vertical features filled with multiple layers of sand that are loosely held together. Thin linings of silt and/or clay separate the margins of dikes as well as layers internal to the dike. Linings are commonly $0.03 \mathrm{~mm}$ to $1.0 \mathrm{~mm}$ in thickness, but linings up to about 10 $\mathrm{mm}$ are known. The width of individual infilling layers ranges from as little as $0.01 \mathrm{~mm}$ to more than 30 $\mathrm{cm}$ and their length can vary from about $0.2 \mathrm{~m}$ to more than $20 \mathrm{~m}$. Infilling sediments are typically poorto well-sorted sand, but may contain clay, silt, and gravel.

Clastic dikes occur in swarms and form four types of networks (Fecht et al. 1999): 1) regular-shaped polygonal patterns, 2) irregular-shaped, polygonal patterns, 3) pre-existing fissure fillings, and 4) random occurrences. In Figure 2.7 an expansive interconnected network of clastic dikes is shown. Unlike most other places in the area, windblown silt and sand don't completely cover flood deposits at this location. Clastic dikes are difficult to see from the ground but are clearly visible on aerial photographs because they contain more fine-grained material than the sediment between the dikes. Dikes stand out in relief and retain more moisture; therefore, they attract different kinds of plants. This contrast in moisture and vegetation is what makes the dikes visible in aerial photographs (Figure 2.7). At the U Tank Farm, clastic dikes probably occur randomly in the GD facies (Hanford formation - unit H1) and may form regularshaped polygons in the SD and ISSD facies (Hanford formation - unit H2). 


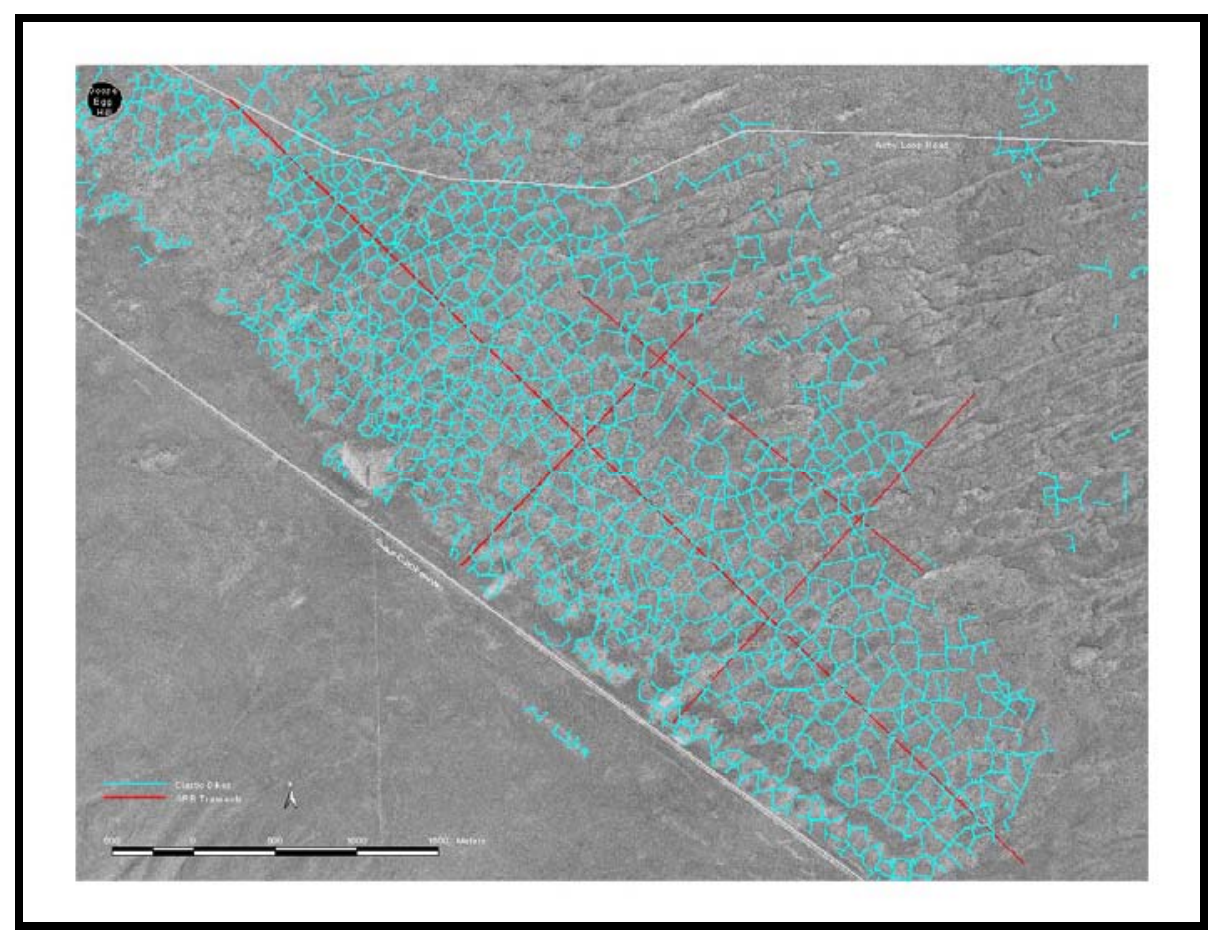

Figure 2.7. A Polygonal, Clastic Dike Network Exposed atop the Deflated Surface of the Hanford Formation (SD facies). Enhanced aerial photograph clearly shows interconnected network of regularly spaced, multisided clastic dikes. Clastic-dike polygons (blue) disappear under a cover of more recent sand dunes, which are younger than the floods, in the upper right. Highway 240 runs diagonally across lower left corner of image.

\subsubsection{Holocene Deposits and Backfill}

Holocene-aged deposits in the 200 West Area are dominated by eolian sand. These sands tend to consist of very fine- to medium-grained, and occasionally silty sands. Eolian deposits were removed from the U Tank Farm during construction of the tank farm. The tank farms were excavated to a depth of about $35 \mathrm{ft}$ during construction and backfilled with silt, sand, and gravel of the Hanford formation and eolian sand.

\subsection{Interpreted Geology of U Tank Farm Direct-Push Boreholes}

All but two of the 89 direct-push samples reported herein were collected from sand-dominated (SD) and interbedded sand- to silt-dominated (ISSD) facies of the $\mathrm{H} 2$ unit within the Hanford formation. Most of the zones targeted for sampling were from short intervals of elevated neutron moisture shown on geophysical logs and appear to be associated with finer grained slackwater sediments at the tops of graded flood rhythmites. This is confirmed by the core samples recovered with the cone penetrometer as well as from continuous core recovered from adjacent 299-W19-44. Finer-grained sediments are almost always more moist than their coarser-grained counterparts from the rhythmite bases. The finer-grained rhythmite tops are known to result in increased sorption and lateral migration of moisture within the vadose zone. Thus, the regularly spiked nature displayed in the neutron-moisture logs below $50 \mathrm{ft}$ is a function of the 
rhythmically bedded nature and abundant fine-grained sand and silt within the Hanford formation $\mathrm{H} 2$ unit. Moisture and contaminants moving through the vadose zone have been shown to collect along these highly contrasting lithologic boundaries.

Two of the samples came from the base of the gravelly Hanford formation $\mathrm{H} 1$ unit. One of these samples, from C9994A, was too coarse grained to provide a decent sample while the other, from C5596, recovered a relatively good sample.

Based on the downhole total gamma and neutron moisture logs, the contact between the $\mathrm{H} 1$ and $\mathrm{H} 2$ units of the Hanford formation appears to lie between 50 and $54 \mathrm{ft}$. This contact was sampled significantly more (up to eight times more) than any other interval for this study. At this contact, not only is there a consistent rise in total gamma activity (Randall and Price 2007), but also a prominent spike in neutron moisture, probably due to a capillary boundary created along the sharp lithologic contact (Figure 2.8). The depth for the $\mathrm{H} 1 / \mathrm{H} 2$ contact is consistent with the contact identified in the adjacent background hole (299-W19-44). Numerous other spikes in neutron moisture below $50 \mathrm{ft}$, without any significant increase in gamma activity, are attributed to higher moisture retention associated with the siltrich tops of graded rhythmites. 


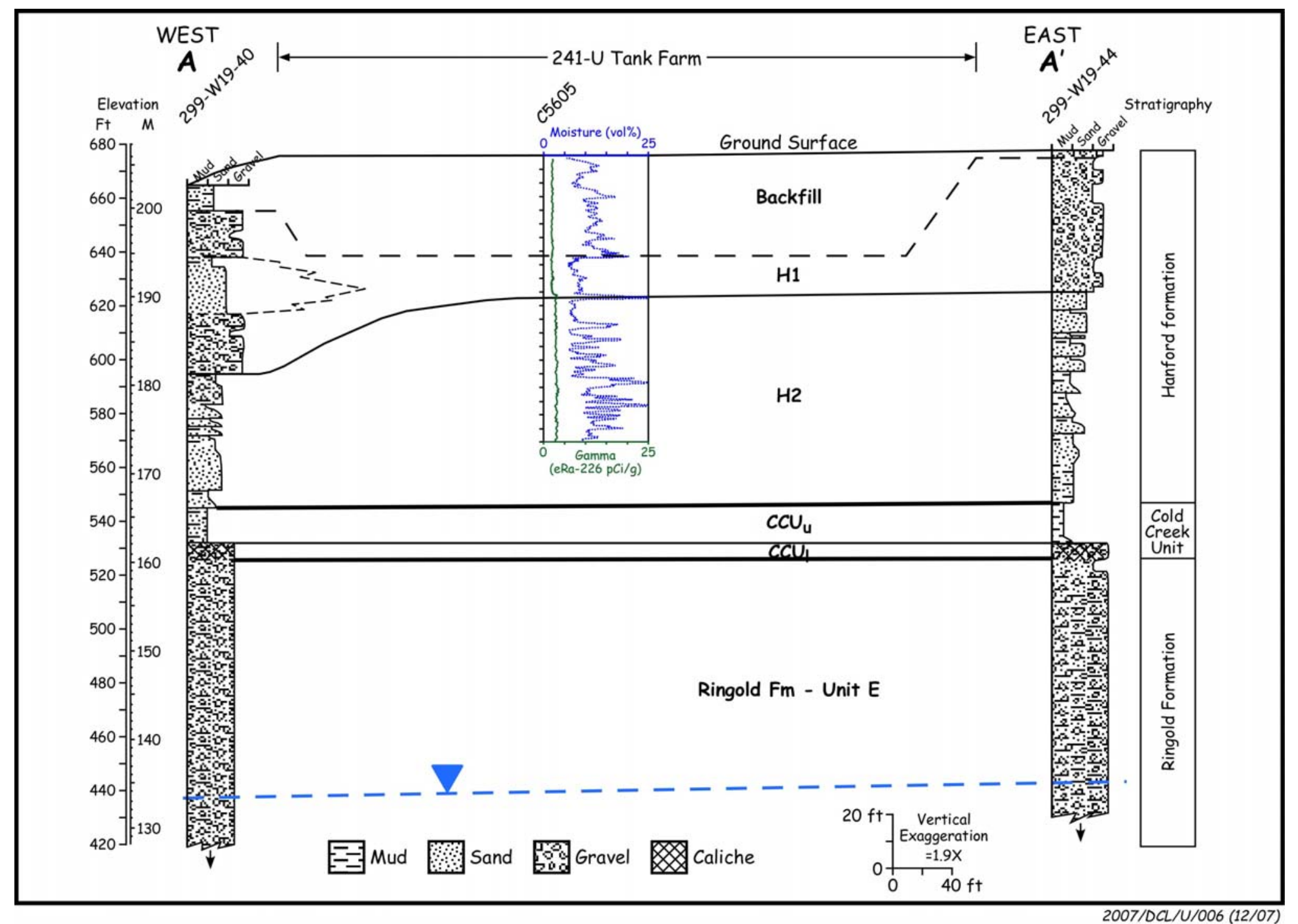

Figure 2.8. Detailed Hydrogeologic Cross Section (A - A') of the Vadose Zone Beneath U Tank Farm. Location of cross section is presented in Figure 2.2. 


\subsection{Geochemical Methods and Materials}

This chapter discusses the methods and philosophy used to characterize the U Tank Farm vadose zone samples and the parameters that were measured and analyzed in the laboratory. It also describes the materials and methods used to conduct analyses of the physical, geochemical, and radio-analytical properties of the sediments.

\subsection{Sample Inventory}

\subsubsection{Background Borehole (C3393) Vadose Zone Samples}

Samples were numbered using a project-specific prefix, in this case C3393 for the background samples collected near the U Tank Farm, followed by a specific sample identification suffix, such as -3 . In this case, the suffix represents the starting depth (measured below ground surface, or bgs) from which the sample was collected. Nearly continuous core (115 samples) was collected from the background borehole at depths from 3 to $144.5 \mathrm{ft}$ bgs. The sediment was collected in lexan liners with approximate dimensions of 4 inches wide by 12 inches long. Of these 115 samples, 18 samples were selected for detailed characterization and analysis (Table 3.1). Two laboratory duplicate samples were collected during core opening; these are designated by the nomenclature DUP.

\subsubsection{U Tank Farm Direct Push Samples}

At the U Tank Farm, sediment samples were collected from ten direct push holes (see Figure 3.1). Each direct push sampling interval resulted in up to three depth-discrete cores (1 to 1.25 inches in diameter by 6 inches long) and one grab sample consisting of the material captured in the drive shoe. Each sample interval collected within the U Tank Farm was numbered using Hanford Environmental Information System (HEIS)-specific sample names. The core samples from each sample interval were further identified by the letters $\mathrm{A}, \mathrm{B}$, or $\mathrm{C}$, where the A liner was always in the deeper position closest to the drive shoe. Three laboratory duplicate samples were collected during core opening; these are designated by the nomenclature DUP. Recovery of samples was fairly good in most of the push holes. The one exception was push hole C5594A, which only had material recovered from the shoe. Details about the U Tank Farm direct push samples are listed in Table 3.2. 
Table 3.1. Sample Inventory from the U Tank Farm Background Borehole

\begin{tabular}{|c|c|c|c|c|c|}
\hline $\begin{array}{l}\text { Sample } \\
\text { Number }\end{array}$ & $\begin{array}{c}\begin{array}{c}\text { Depth } \\
\text { (ft bgs) }\end{array} \\
\end{array}$ & $\begin{array}{l}\text { Sample } \\
\text { Number }\end{array}$ & $\begin{array}{c}\begin{array}{c}\text { Depth } \\
\text { (ft bgs) }\end{array} \\
\end{array}$ & $\begin{array}{l}\text { Sample } \\
\text { Number }\end{array}$ & $\begin{array}{c}\text { Depth } \\
\text { (ft bgs) }\end{array}$ \\
\hline C3393-3 & 3.5 & C3393-50 & 50.5 & C3393-101 & 101.5 \\
\hline C3393-4 & 4.5 & C3393-51 & 51.5 & C3393-102.5 & 103.0 \\
\hline C3393-5 & 5.5 & C3393-52.5 & 53.0 & C3393-103.5 & 104.0 \\
\hline C3393-6 & 6.5 & C3393-53.5 & 54.0 & C3393-103.5 Dup & 104.0 \\
\hline C3393-7.5 & 8.0 & C3393-55 & 55.5 & C3393-105 & 105.5 \\
\hline C3393-8.5 & 9.0 & C3393-56 & 56.5 & C3393-106 & 106.5 \\
\hline C3393-10.5 & 11.0 & C3393-57.5 & 58.0 & C3393-107.5 & 108.0 \\
\hline C3393-11.5 & 12.0 & C3393-58.5 & 59.0 & C3393-108.5 & 109.0 \\
\hline C3393-13 & 13.5 & C3393-60 & 60.5 & C3393-108.5 Dup & 109.0 \\
\hline C3393-14 & 14.5 & C3393-61 & 61.5 & C3393-110 & 110.5 \\
\hline C3393-15.5 & 16.0 & C3393-62.5 & 63.0 & C3393-111 & 111.5 \\
\hline C3393-16.5 & 17.0 & C3393-63.5 & 64.0 & C3393-112.5 & 113.0 \\
\hline C3393-17.5 & 18.0 & C3393-65 & 65.5 & C3393-113.5 & 114.0 \\
\hline C3393-18.5 & 19.0 & C3393-66 & 66.5 & C3393-115 & 115.5 \\
\hline C3393-19.3 & 19.0 & C3393-67.5 & 68.0 & C3393-116 & 116.5 \\
\hline C3393-20 & 20.5 & C3393-68.5 & 69.0 & C3393-117.5 & 118.0 \\
\hline C3393-21 & 21.5 & C3393-70 & 70.5 & C3393-118.5 & 119.0 \\
\hline C3393-22.5 & 23.0 & C3393-71 & 71.5 & C3393-120 & 120.5 \\
\hline C3393-23.5 & 24.0 & C3393-72.5 & 73.0 & C3393-121 & 121.5 \\
\hline C3393-25.5 & 26.0 & C3393-73.5 & 74.0 & C3393-122.5 & 123.0 \\
\hline C3393-26.5 & 27.0 & C3393-75 & 75.5 & C3393-123.5 & 124.0 \\
\hline C3393-28 & 28.5 & C3393-76 & 76.5 & C3393-125 & 125.5 \\
\hline C3393-29 & 29.5 & C3393-77.5 & 78.0 & C3393-126 & 126.5 \\
\hline C3393-30 & 30.5 & C3393-78.5 & 79.0 & C3393-127.5 & 128.0 \\
\hline C3393-31 & 31.5 & C3393-82.5 & 83.0 & C3393-128.5 & 129.0 \\
\hline C3393-33 & 33.5 & C3393-83.5 & 84.0 & C3393-130 & 130.5 \\
\hline C3393-34 & 34.5 & C3393-85 & 85.5 & C3393-131 & 131.5 \\
\hline C3393-35.5 & 36.0 & C3393-86 & 86.5 & C3393-132.5 & 133.0 \\
\hline C3393-36.5 & 37.0 & C3393-87.5 & 88.0 & C3393-133.5 & 134.0 \\
\hline C3393-37.5 & 38.0 & C3393-88.5 & 89.0 & C3393-135 & 135.5 \\
\hline C3393-38.5 & 39.0 & C3393-90 & 90.5 & C3393-136 & 136.5 \\
\hline C3393-40 & 40.5 & C3393-91 & 91.5 & C3393-137.5 & 138.0 \\
\hline C3393-41 & 41.5 & C3393-92.5 & 93.0 & C3393-138.5 & 139.0 \\
\hline C3393-42.5 & 43.0 & C3393-93.5 & 94.0 & C3393-140 & 140.5 \\
\hline C3393-43.5 & 44.0 & C3393-95 & 95.5 & C3393-141 & 141.5 \\
\hline C3393-45 & 45.5 & C3393-96 & 96.5 & C3393-142.5 & 143.0 \\
\hline C3393-46 & 46.5 & C3393-97.5 & 98.0 & C3393-143.5 & 144.0 \\
\hline C3393-47.5 & 48.0 & C3393-98.5 & 99.0 & & \\
\hline C3393-48.5 & 49.0 & C3393-100 & 100.5 & & \\
\hline
\end{tabular}


Table 3.2. Sample Inventory from the U Tank Farm Direct Push Holes

\begin{tabular}{|c|c|c|c|c|c|}
\hline $\begin{array}{l}\text { Sample } \\
\text { Number }\end{array}$ & $\begin{array}{c}\text { Probe Hole } \\
\text { Number }\end{array}$ & $\begin{array}{l}\begin{array}{c}\text { Depth } \\
\text { (ft bgs) }\end{array} \\
\end{array}$ & $\begin{array}{c}\text { Sample } \\
\text { Number } \\
\end{array}$ & $\begin{array}{c}\text { Probe Hole } \\
\text { Number }\end{array}$ & $\begin{array}{c}\begin{array}{c}\text { Depth } \\
\text { (ft bgs) }\end{array} \\
\end{array}$ \\
\hline "B1NDW3C & C5590 & "95.8 & "B1NTD5C & "C5600 & 81.8 \\
\hline B1NDW3B & C5590 & 96.3 & B1NTD5B & C5600 & 82.3 \\
\hline B1NDW3A & C5590 & 96.8 & B1NTD5A & C5600 & 82.8 \\
\hline B1NDW3 & C5590 & 97.3 & B1NTD5 & C5600 & 83.3 \\
\hline B1NHV0C & C5592 & 61.8 & B1P1K6C & C5600 & 88.3 \\
\hline B1NHV0B & C5592 & 62.3 & B1P1K6B & C5600 & 88.8 \\
\hline B1NHV0A & C5592 & 62.8 & B1P1K6A & C5600 & 89.3 \\
\hline B1NHV0 & C5592 & 63.3 & B1P1K6 & C5600 & 89.8 \\
\hline B1NDW4 & C5594A & 57.3 & B1P3F9C & C5604 & 50.3 \\
\hline B1NTC6C & C5598 & 49.8 & B1P3F9B & C5604 & 50.8 \\
\hline B1NTC6B & C5598 & 50.3 & B1P3F9A & C5604 & 51.3 \\
\hline B1NTC6A & C5598 & 50.8 & B1P3F9 & C5604 & 51.8 \\
\hline B1NTC6 & C5598 & 51.3 & B1P3H0C & C5602 & 51.3 \\
\hline B1NTC7C & C5598 & 59.3 & B1P3H0B & C5602 & 51.8 \\
\hline B1NTC7B & C5598 & 59.8 & B1P3H0A & C5602 & 52.3 \\
\hline B1NTC7A & C5598 & 60.3 & B1P3H0 & C5602 & 52.8 \\
\hline B1NTC7 & C5598 & 60.8 & B1P3H1C & C5602 & 67.3 \\
\hline B1NTC8C & C5598 & 81.8 & B1P3H1B & C5602 & 67.8 \\
\hline B1NTC8B & C5598 & 82.3 & B1P3H1A & C5602 & 68.3 \\
\hline B1NTC8A & C5598 & 82.8 & B1P3H1 & C5602 & 68.8 \\
\hline B1NTC8 & C5598 & 83.3 & B1P3H2C & C5602 & 82.3 \\
\hline B1NTC9C & C5596 & 50.3 & B1P3H2B & C5602 & 82.8 \\
\hline B1NTC9B & C5596 & 50.8 & B1P3H2A & C5602 & 83.3 \\
\hline B1NTC9A & C5596 & 51.3 & B1P3H2 & C5602 & 83.8 \\
\hline B1NTC9 & C5596 & 51.8 & B1PBB0C & C5602 & 91.3 \\
\hline B1NTD0C & C5596 & 60.8 & B1PBB0B & C5602 & 91.8 \\
\hline B1NTD0B & C5596 & 61.3 & B1PBB0A & C5602 & 92.3 \\
\hline B1NTD0A & C5596 & 61.8 & B1PBB0 & C5602 & 92.8 \\
\hline B1NTD0 & C5596 & 62.3 & B1PBB1C & C5606 & 51.3 \\
\hline B1NTD1C & C5596 & 77.3 & B1PBB1B & C5606 & 51.8 \\
\hline B1NTD1B & C5596 & 77.8 & B1PBB1A & C5606 & 52.3 \\
\hline B1NTD1A & C5596 & 78.3 & B1PBB1 & C5606 & 52.8 \\
\hline B1NTD1 & C5596 & 78.8 & B1PK51C & C5608 & 63.8 \\
\hline B1NTD2C & C5596 & 82.3 & B1PK51B & C5608 & 64.3 \\
\hline B1NTD2B & C5596 & 82.8 & B1PK51A & C5608 & 64.8 \\
\hline B1NTD2A & C5596 & 83.3 & B1PK51 & C5608 & 65.3 \\
\hline B1NTD2 & C5596 & 83.8 & B1PK52C & C5608 & 85.3 \\
\hline B1NTD3C & C5600 & 49.8 & B1PK52B & C5608 & 85.8 \\
\hline B1NTD3B & C5600 & 50.3 & B1PK52A & C5608 & 86.3 \\
\hline B1NTD3A & C5600 & 50.8 & B1PK52 & C5608 & 86.8 \\
\hline B1NTD3 & C5600 & 51.3 & B1PK53C & C5608 & 97.3 \\
\hline B1NTD4C & C5600 & 59.8 & B1PK53B & C5608 & 97.8 \\
\hline B1NTD4B & C5600 & 60.3 & B1PK53A & C5608 & 98.3 \\
\hline B1NTD4A & C5600 & 60.8 & B1PK53 & C5608 & 98.8 \\
\hline B1NTD4 & C5600 & 61.3 & & & \\
\hline
\end{tabular}




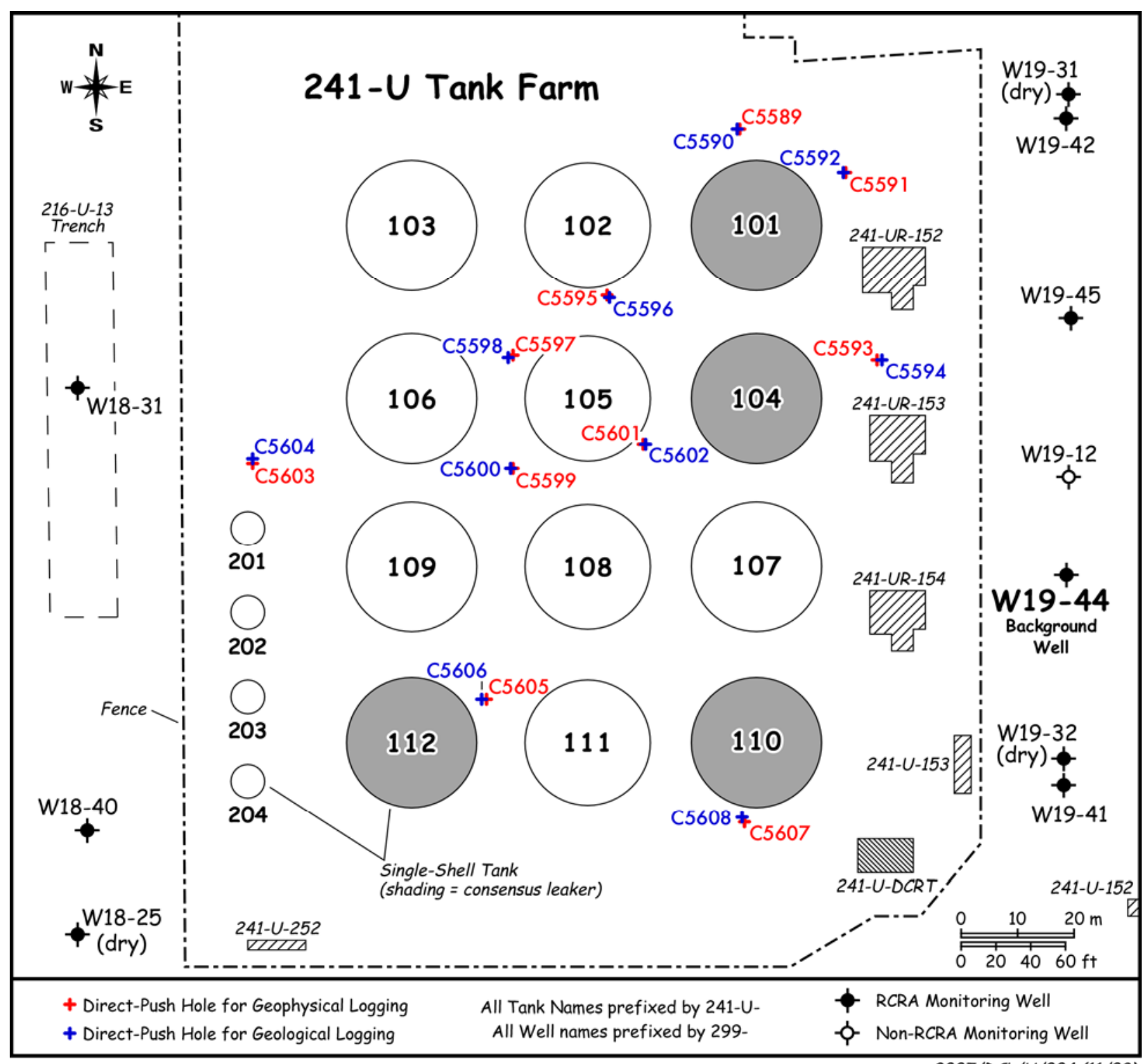

2007/DCL/U/004 (11/08)

Figure 3.1. Location Map of U Tank Farm

\subsection{Approach}

During a past investigation at WMA SX, it was found that changes in sediment type and contaminant concentrations often occurred within a distance of a few inches within a given liner (Serne et al. 2002b). It was concluded that a more methodical scoping approach would be necessary to provide the technical justification for selecting samples for detailed characterization as defined in the data quality objectives process (DOE 1999). Subsequently, a method was developed to select samples that considered depth, geology (e.g., lithology, grain-size composition, and carbonate content, etc.), individual liner contaminant concentration (e.g., radionuclides, nitrate), moisture content, and overall sample quality. Extraction and leaching procedures were performed and certain key parameters (i.e., moisture content, gamma energy 
analysis) were measured on sediment from the liners. Grab samples were only utilized as part of this study if sufficient sample material for characterization and analysis was not contained in the core samples.

During the geologic examination of the core samples, the liner contents were sub-sampled for moisture content, gamma-emission radiocounting, 1:1 water extracts (which provide soil $\mathrm{pH}$, electrical conductivity (EC), cation, and anion data), total carbon and inorganic carbon content, and $8 \mathrm{M}$ nitric acid extracts (which provide a measure of the total leachable sediment content of the contaminants). Sampling preference was always biased toward the finer-grained and/or wetter material contained in each liner. It has been our experience that elevated concentrations of contaminants occur in sediment fractions characterized by higher water contents. The remaining sediment from each liner was then sealed and placed in cold storage.

\subsection{Materials and Methods}

During sub-sampling, every effort was made to minimize moisture loss and prevent cross contamination between samples. Depending on the sample matrix, very coarse pebbles and larger material (i.e., $>32 \mathrm{~mm}$ ) were avoided during sub-sampling. Larger substrate was excluded to provide moisture contents representative of gamma energy analysis and 1:1 sediment:water extract samples. Therefore, the results from the sub-sample measurements may contain a possible bias toward higher concentrations for some analytes that would be preferentially associated with the smaller sized sediment fractions.

Procedures ASTM D2488-93 (1993) and PNL-MA-567-DO-1 (PNL 1990) were followed for visual descriptions and geological descriptions of all samples. The sediment classification scheme used for geologic identification of the sediment types (used solely for graphing purposes in this report) was based on the modified Folk/Wentworth classification scheme (1968/1922).

\subsubsection{Moisture Content}

Gravimetric water contents of the sediment samples were determined using PNNL procedure PNNLAGG-WC-001 (PNNL 2005). This procedure is based on the American Society for Testing and Materials procedure "Test Method for Laboratory Determination of Water (Moisture) Content of Soil and Rock by Mass" (ASTM D2216-98 [ASTM 1998]). One representative sub-sample of at least 15 to $70 \mathrm{~g}$ was used. Sediment aliquots were placed in tared containers, weighed, and dried in an oven at $105^{\circ} \mathrm{C}$ until constant weight was achieved, which took at least 24 hours. The containers were removed from the oven, sealed, cooled, and weighed. At least two weighings, each after a 24-hour heating period, were performed to ensure that all moisture was removed. All weighings were performed using a calibrated balance. A calibrated weight set was used to verify balance performance before weighing the samples. The gravimetric water content was computed as the percentage change in soil weight before and after oven drying.

\section{1:1 Sediment:Water Extracts}

Water-soluble inorganic constituents were determined using a 1:1 sediment:deionized-water extract method. The extracts were prepared by adding an exact weight of deionized water to approximately 60 to $80 \mathrm{~g}$ of sediment sub-sampled from each liner. The weight of deionized water needed was calculated based on the weight of the field-moist samples and their previously determined moisture contents. The 
sum of the existing moisture (pore water) and the deionized water was fixed at the mass of the dry sediment. An appropriate amount of deionized water was added to screw cap jars containing the sediment samples. The jars were sealed and briefly shaken by hand, then placed on a mechanical orbital shaker for one hour. The samples were allowed to settle, generally overnight, until the supernatant liquid was fairly clear. The supernatant was carefully decanted, filtered (passed through $0.45 \mu \mathrm{m}$ membranes) and analyzed for conductivity, $\mathrm{pH}$, anions, cations, alkalinity, and radionuclide analyses. More details can be found in Rhoades (1996) and within Methods of Soils Analysis - Part 3 (ASA 1996).

\subsubsection{1 pH and Conductivity}

Two aliquots of approximately 3-mL volume of the 1:1 sediment:water extract supernatants were used for $\mathrm{pH}$ and conductivity measurements. The $\mathrm{pH}$ of the extracts was measured with a solid-state $\mathrm{pH}$ electrode and a pH meter calibrated with buffers 4, 7, and 10. Electrical conductivity was measured and compared to potassium chloride standards with a range of $0.001 \mathrm{M}$ to $1.0 \mathrm{M}$.

\subsubsection{Anions}

The 1:1 sediment:water extracts were analyzed for anions using ion chromatography (IC). Fluoride, chloride, nitrite, bromide, nitrate, carbonate, phosphate, and sulfate were separated on a Dionex AS17 column with a gradient elution of $1 \mathrm{mM}$ to $35 \mathrm{mM}$ sodium hydroxide and measured using a conductivity detector. This methodology is based on U.S. Environmental Protection Agency (EPA) Method 300.0A (EPA 1984) with the exception of using the gradient elution of sodium hydroxide.

\subsubsection{Cations and Trace Metals}

Major cation analysis was performed using an inductively coupled plasma-optical emission spectroscopy (ICP-OES) unit using high-purity calibration standards to generate calibration curves and verify continuing calibration during the analytical run. Multiple dilutions were made of each 1:1 water extract to investigate and correct for matrix interferences. Details of this method are found in EPA Method 6010B (EPA 2000b). The second instrument used to analyze trace metals, including technetium99 and uranium-238, was an inductively coupled plasma-mass spectrometer (ICP-MS) using PNNLAGG-415 method (PNNL 1998). This method is similar to EPA Method 6020 (EPA 2000c).

\subsubsection{Alkalinity}

The alkalinity of several of the 1:1 sediment:water extracts was measured using standard titration. The alkalinity procedure is equivalent to the U.S. Geological Survey (USGS) National Field Manual (USGS 2001) method.

\subsection{3 $\quad 8$ M Nitric Acid Extract}

Approximately $20 \mathrm{~g}$ of oven-dried sediment was contacted with $8 \mathrm{M}$ nitric acid at a ratio of approximately five parts acid to one part sediment. The slurries were heated to about $80^{\circ} \mathrm{C}$ for several hours, then the fluid was separated by filtration through $0.45 \mu \mathrm{m}$ membranes. The acid extracts were analyzed for major cations and trace metals using ICP-OES and ICP-MS techniques, respectively. The acid digestion procedure is based on EPA SW-846 Method 3050B (EPA 2000a). 


\subsubsection{Gamma Energy Analysis}

Gamma energy analysis (GEA) was performed on sediment from the background borehole and direct push liners. All samples for GEA were analyzed using $60 \%$ efficient intrinsic germanium gamma detectors. All germanium counters were efficiency calibrated for distinct geometries using mixed gamma standards traceable to the National Institute of Standards and Technology (NIST). Field-moist samples were placed in $150-\mathrm{cm}^{3}$ counting containers and analyzed for 100 minutes in a fixed geometry. All spectra were background-subtracted. Spectral analysis was conducted using libraries containing most mixed fission products, activation products, and natural decay products. Control samples were run throughout the analysis to ensure correct operation of the detectors. The controls contained isotopes with photo peaks spanning the full detector range and were monitored for peak position, counting rate, and full-width half-maximum. Details are found in Gamma Energy Analysis, Operation, and Instrument Verification using Genie2000 ${ }^{\text {TM }}$ Support Software (PNNL 1997).

\subsubsection{Total Beta and Total Alpha Measurements on Water and Acid Extracts}

Gross alpha and beta measurements were made on both the water and acid extracts from the direct push samples only (e.g., the background borehole samples were not analyzed for total alpha and beta). For each extract, approximately $1 \mathrm{~mL}$ of sample was placed in a $20-\mathrm{mL}$ tared liquid scintillation vial and weighed. Fifteen $\mathrm{mL}$ of scintillation cocktail were then added and the samples were mixed and counted on a Wallac Model 1415 Liquid Scintillation Counter as prescribed in procedure AGG-RRL-002, Liquid Scintillation Counting and Instrument Verification Using the 1400 DSA ${ }^{\mathrm{TM}}$ Support Software (PNNL 2000). Results were converted to picocuries (pCi) per gram of dry sediment by using the known solutionto-solid ratios used to extract aliquots of the sediment.

\subsubsection{Carbon Content of Sediment}

The total carbon concentration in aliquots of sediment from the background borehole and core liners was measured with a Shimadzu TOC-V CSN instrument with a SSM-5000A Total Organic Carbon Analyzer by combustion at approximately $900^{\circ} \mathrm{C}$ based on ASTM Method, Standard Test Methods for Analysis of Metal Bearing Ores and Related Materials by Combustion Infrared Absorption Spectrometry (ASTM E1915-01 2001). Samples were placed into pre-combusted, tared, ceramic combustion sample holders and weighed on a calibrated balance. After the combustion sample holders were placed into the furnace introduction tube, an approximately 2-minute waiting period was allowed for the ultra-pure oxygen carrier gas to remove any carbon dioxide introduced to the system from the atmosphere during sample placement. After this sparging process, the sample was moved into the furnace and the combustion was begun. The carrier gas then delivered the sample combustion products to the cell of a non-dispersive infrared (NDIR) gas analyzer where the carbon dioxide was detected and measured. The amount of $\mathrm{CO}_{2}$ measured is proportional to the total carbon content of the sample. Adequate system performance was confirmed by analyzing known quantities of a calcium carbonate standard.

Sediment samples were analyzed for inorganic carbon content by placing an aliquot of sediment into a ceramic combustion boat. The combustion boat was placed into the sample introduction tube where it was sparged with ultra-pure oxygen for two minutes to remove atmospheric carbon dioxide. A small amount (usually $0.6 \mathrm{ml}$ ) of $3 \mathrm{M}$ phosphoric acid was then added to the sample in the combustion boat. The boat was moved into the combustion furnace where it was heated to $200^{\circ} \mathrm{C}$. Samples were completely covered by the acid to allow full reaction to occur. Ultra-pure oxygen swept the resulting 
carbon dioxide through a dehumidifier and scrubber into the cell of a NDIR gas analyzer where the carbon dioxide was detected and measured. The amount of $\mathrm{CO}_{2}$ measured is proportional to the inorganic carbon content of the sample.

Organic carbon content was determined by the difference between the inorganic carbon and total carbon concentrations.

\subsubsection{Particle-Size Distribution}

Wet sieving and hydrometer methods were used to determine the particle size distribution of selected samples from the background borehole, C3393. No particle size measurements were made on the direct push samples. The hydrometer technique is described in ASA (1986a), Part 1, Method 15-5, Hydrometer Method; it concentrates on quantifying the relative amounts of silt and clay. The silt and clay separates were saved for later mineralogical analyses. Samples from the borehole that were used for the hydrometer method were never air nor oven dried to minimize the effects of particle aggregation that can affect the separation of clay grains from the coarser material.

\subsubsection{Particle Density}

The particle density of bulk grains from the background borehole are usually determined using pychnometers as described in ASA (1986b) Part 1, Method 14-3, Pychnometer Method, and oven-dried

material. The particle density is an input needed to determine the particle size when using the hydrometer method. However, no direct particle density measurements were made for the sediments from borehole C3393. The particle size data reported in this document used the quartz default value of $2.65 \mathrm{~g} / \mathrm{cm}^{3}$ to calculate the particle size distribution. The error in using this simplifying assumption is not significant since most of the samples consisted of fine- to medium-grained sand made up mostly of quartz grains.

\subsubsection{Water Potential (Suction) Measurements}

Suction measurements were made on the core liners in each splitspoon sampler from borehole C3393 using the filter paper method PNL-MA-567-SFA-2 (PNL 1990), which is essentially the same as ASTM (2002). This method relies on three filter papers folded together into a small sandwich that rapidly equilibrates with the sediment sample. The middle filter paper does not contact sediment that might stick to the paper and bias the mass measurements. At equilibrium, the matric suction in the filter paper is the same as the matric suction of the sediment sample. The dry filter paper sandwiches were placed in the borehole C3393 liners while still filled with the sediment, and remained there for 3 weeks to allow sufficient time for the matric suction in the sediment to equilibrate with the matric suction in the filter paper. The mass of the wetted middle filter paper that has had no direct contact with the sediment was subsequently determined, and the suction of the sediment was determined from a calibration relationship between filter paper water content and matric suction. The filter paper method provides a good estimate of water potentials over the range from -0.01 to $-2 \mathrm{MPa}$ (1 to $200 \mathrm{~m}$ [3.3 to $656 \mathrm{ft}$ ] suction head) (Deka et al. 1995). 
The relationships used for converting the water content of filter paper to matric suction for Whatman \#42 filter paper have been determined by Deka et al. (1995) and can be expressed as:

$$
\begin{aligned}
& \mathrm{Sm}=10^{(5.144-6.699 \mathrm{w})} / 10 \text { for } \mathrm{w}<0.5 \\
& \mathrm{Sm}=10^{(2.383-1.309 \mathrm{w})} / 10 \text { for } \mathrm{w}>0.5
\end{aligned}
$$

where: $\mathrm{Sm}=$ matric suction $(\mathrm{m})$

$\mathrm{w}=$ gravimetric water content of the filter paper $(\mathrm{g} / \mathrm{g})$.

Soil matric suction analysis was conducted on 29 core liner samples from borehole C3393. The matric potential samples covered the borehole profile from 4 to $144 \mathrm{ft}$ bgs in approximately $5 \mathrm{ft}$ increments.

\subsubsection{Cation Exchange Capacity Analysis}

The exchangeable fraction of cations present in the sediments was determined using a $1 \mathrm{M}$ ammonium acetate extraction. The extracts were prepared by adding approximately $15 \mathrm{~g}$ of sediment to centrifuge tubes containing approximately $35 \mathrm{ml}$ of $1 \mathrm{M}$ ammonium acetate. The samples were shaken overnight on a mechanical orbital shaker. At the time of sampling, the tubes were placed in a centrifuge and spun at approximately $2200 \mathrm{~g}$ for 10 minutes. Upon removal from the centrifuge, supernatant was withdrawn and filtered using $0.45 \mu \mathrm{m}$ membranes attached to syringes. The filtered samples were analyzed for major cations using ICP-OES. The total amount of cations in solution were summed and used to calculate the total cation exchange capacity of the sediments. 


\subsection{Results and Discussion}

This section presents the geochemical and physical characterization data collected on sediment from the background borehole emplaced adjacent to the U Tank Farm as well as the direct push holes emplaced within the U Tank Farm. The activities employed emphasized tests that provided basic characterization data and were key to determining the distribution of mobile contaminants in the vadose zone sediments. Such information on the vadose zone sediments included moisture content, total and inorganic carbon content, $\mathrm{pH}$, electrical conductivity (EC), and measurements of major cations, anions, and trace metals (including technetium-99 and uranium-238) in 1:1 sediment:water and $8 \mathrm{M}$ nitric acid extracts. Gamma energy analysis (GEA) of the sediments was also performed to search for any detectable anthropogenic gamma-emitting radionuclides.

\subsection{Vadose Zone Sediment from the Background Borehole Samples (C3393)}

\subsubsection{Moisture Content}

The moisture contents of the 113 core liners collected from the U Farm background borehole are listed as a function of depth in Table 4.1. The moisture content profile correlates with the lithology described in Section 2 and presented in Table 2.3. One region of elevated moisture occurred in the Hanford formation $\mathrm{H} 1 \mathrm{unit}$; it was described as a fine-medium sand lens at $\sim 6.5 \mathrm{ft}$ bgs and had a moisture content of $20.3 \%$. The rest of the Hanford formation $\mathrm{H} 1$ unit was rather dry, with a mean gravimetric moisture content of $3.93 \mathrm{wt} \%$. The next zone of elevated moisture was found within the Hanford formation $\mathrm{H} 2$ unit at $\sim 58 \mathrm{ft}$ bgs, with a gravimetric moisture content of $14.7 \mathrm{wt} \%-18.3 \mathrm{wt} \%$. Several other zones of elevated moisture were found throughout the Hanford formation $\mathrm{H} 2$ unit; they all contained fine sand and/or silt and had moisture contents that ranged from $10.8 \mathrm{wt} \%$ to $17.2 \mathrm{wt} \%$. Below the Hanford formation strata, the Cold Creek upper sub-unit $\left(\mathrm{CCU}_{u}\right)$ was penetrated by the final ten splitspoon core samples collected. The $\mathrm{CCU}_{\mathrm{u}}$ was much moister than the overlying Hanford formation units, with an average moisture content of $16.4 \mathrm{wt} \%$.

Table 4.1. Gravimetric Moisture Content of Samples Obtained from the U Tank Farm Background Borehole

\begin{tabular}{|l|c|c|c||}
\hline \multicolumn{1}{|c|}{ Sample ID } & Mid-Depth (ft bgs) & Stratigraphic Unit & Moisture (\%) \\
\hline \hline C3393-3 & 3.5 & H1 & $4.05 \%$ \\
\hline C3393-4 & 4.5 & H1 & $4.95 \%$ \\
\hline C3393-5 & 5.5 & H1 & $4.92 \%$ \\
\hline C3393-6 & 6.5 & H1 & $20.3 \%$ \\
\hline C3393-7.5 & 8.0 & H1 & $3.66 \%$ \\
\hline C3393-8.5 & 9.0 & H1 & $3.63 \%$ \\
\hline C3393-10.5 & 11.0 & H1 & $2.99 \%$ \\
\hline C3393-11.5 & 12.0 & H1 & $2.92 \%$ \\
\hline C3393-13 & 13.5 & H1 & $3.24 \%$ \\
\hline C3393-14 & 14.5 & H1 & $3.41 \%$ \\
\hline C3393-15.5 & 16.0 & H1 & $3.45 \%$ \\
\hline C33393-16.5 & 17.0 & H1 & $4.13 \%$ \\
\hline C3393-17.5 & 18.0 & H1 & $3.33 \%$ \\
\hline C3393-18.5 & 19.0 & H1 & $4.19 \%$ \\
\hline
\end{tabular}




\begin{tabular}{|c|c|c|c|}
\hline Sample ID & Mid-Depth (ft bgs) & Stratigraphic Unit & Moisture (\%) \\
\hline C3393-19.25 & 19.0 & בH1 & $6.31 \%$ \\
\hline C3393-20 & 20.5 & $\mathrm{H} 1$ & $2.65 \%$ \\
\hline C3393-21 & 21.5 & H1 & $2.93 \%$ \\
\hline C3393-22.5 & 23.0 & H1 & $8.34 \%$ \\
\hline C3393-23.5 & 24.0 & H1 & $3.87 \%$ \\
\hline C3393-25.5 & 26.0 & $\mathrm{H} 1$ & $2.91 \%$ \\
\hline C3393-26.5 & 27.0 & $\mathrm{H} 1$ & $3.56 \%$ \\
\hline C3393-28 & 28.5 & $\mathrm{H} 1$ & $3.74 \%$ \\
\hline C3393-29 & 29.5 & H1 & $4.23 \%$ \\
\hline C3393-30 & 30.5 & $\mathrm{H} 1$ & $2.59 \%$ \\
\hline C3393-31 & 31.5 & H1 & $4.26 \%$ \\
\hline C3393-33 & 33.5 & H1 & $3.90 \%$ \\
\hline C3393-34 & 34.5 & H1 & $3.82 \%$ \\
\hline C3393-35.5 & 36.0 & H1 & $3.29 \%$ \\
\hline C3393-36.5 & 37.0 & H1 & $3.62 \%$ \\
\hline C3393-37.5 & 38.0 & $\mathrm{H} 1$ & $4.23 \%$ \\
\hline C3393-38.5 & 39.0 & H1 & $3.46 \%$ \\
\hline C3393-40 & 40.5 & H1 & $3.47 \%$ \\
\hline C3393-41 & 41.5 & $\mathrm{H} 1$ & $3.58 \%$ \\
\hline C3393-42.5 & 43.0 & $\mathrm{H} 1$ & $3.97 \%$ \\
\hline C3393-43.5 & 44.0 & $\mathrm{H} 1$ & $3.90 \%$ \\
\hline C3393-45 & 45.5 & $\mathrm{H} 1$ & $4.56 \%$ \\
\hline C3393-46 & 46.5 & $\mathrm{H} 1$ & $3.88 \%$ \\
\hline C3393-47.5 & 48.0 & H1 & $5.04 \%$ \\
\hline C3393-48.5 & 49.0 & $\mathrm{H} 1$ & $4.36 \%$ \\
\hline C3393-50 & 50.5 & $\mathrm{H} 2$ & $3.19 \%$ \\
\hline C3393-51 & 51.5 & $\mathrm{H} 2$ & $2.65 \%$ \\
\hline C3393-52.5 & 53.0 & $\mathrm{H} 2$ & $3.58 \%$ \\
\hline C3393-53.5 & 54.0 & $\mathrm{H} 2$ & $3.48 \%$ \\
\hline C3393-55 & 55.5 & $\mathrm{H} 2$ & $2.74 \%$ \\
\hline C3393-56 & 56.5 & $\mathrm{H} 2$ & $2.50 \%$ \\
\hline C3393-57.5 & 58.0 & $\mathrm{H} 2$ & $14.7 \%$ \\
\hline C3393-58.5 & 59.0 & $\mathrm{H} 2$ & $18.3 \%$ \\
\hline C3393-60 & 60.5 & $\mathrm{H} 2$ & $2.55 \%$ \\
\hline C3393-61 & 61.5 & $\mathrm{H} 2$ & $2.10 \%$ \\
\hline C3393-62.5 & 63.0 & $\mathrm{H} 2$ & $2.84 \%$ \\
\hline C3393-63.5 & 64.0 & $\mathrm{H} 2$ & $2.19 \%$ \\
\hline C3393-65 & 65.5 & $\mathrm{H} 2$ & $2.86 \%$ \\
\hline C3393-66 & 66.5 & $\mathrm{H} 2$ & $3.57 \%$ \\
\hline C3393-67.5 & 68.0 & $\mathrm{H} 2$ & $3.90 \%$ \\
\hline C3393-68.5 & 69.0 & $\mathrm{H} 2$ & $3.34 \%$ \\
\hline C3393-70 & 70.5 & $\mathrm{H} 2$ & $4.14 \%$ \\
\hline C3393-71 & 71.5 & $\mathrm{H} 2$ & $2.35 \%$ \\
\hline C3393-72.5 & 73.0 & $\mathrm{H} 2$ & $4.57 \%$ \\
\hline C3393-73.5 & 74.0 & $\mathrm{H} 2$ & $3.23 \%$ \\
\hline C3393-75 & 75.5 & $\mathrm{H} 2$ & $3.87 \%$ \\
\hline C3393-76 & 76.5 & $\mathrm{H} 2$ & $11.9 \%$ \\
\hline C3393-77.5 & 78.0 & $\mathrm{H} 2$ & $7.43 \%$ \\
\hline C3393-78.5 & 79.0 & $\mathrm{H} 2$ & $3.44 \%$ \\
\hline C3393-82.5 & 83.0 & $\mathrm{H} 2$ & $5.34 \%$ \\
\hline C3393-83.5 & 84.0 & $\mathrm{H} 2$ & $7.13 \%$ \\
\hline
\end{tabular}




\begin{tabular}{|c|c|c|c|}
\hline Sample ID & Mid-Depth (ft bgs) & Stratigraphic Unit & Moisture (\%) \\
\hline "C3393-85 & 85.5 & $\mathrm{H} 2$ & $6.34 \%$ \\
\hline C3393-86 & 86.5 & $\mathrm{H} 2$ & $11.6 \%$ \\
\hline C3393-87.5 & 88.0 & $\mathrm{H} 2$ & $5.01 \%$ \\
\hline C3393-88.5 & 89.0 & $\mathrm{H} 2$ & $3.78 \%$ \\
\hline C3393-90 & 90.5 & $\mathrm{H} 2$ & $2.97 \%$ \\
\hline C3393-91 & 91.5 & $\mathrm{H} 2$ & $15.9 \%$ \\
\hline C3393-92.5 & 93.0 & $\mathrm{H} 2$ & $4.40 \%$ \\
\hline C3393-93.5 & 94.0 & $\mathrm{H} 2$ & $6.42 \%$ \\
\hline C3393-95 & 95.5 & $\mathrm{H} 2$ & $14.5 \%$ \\
\hline C3393-96 & 96.5 & $\mathrm{H} 2$ & $10.8 \%$ \\
\hline C3393-97.5 & 98.0 & $\mathrm{H} 2$ & $7.63 \%$ \\
\hline C3393-98.5 & 99.0 & $\mathrm{H} 2$ & $8.45 \%$ \\
\hline C3393-100 & 100.5 & $\mathrm{H} 2$ & $2.77 \%$ \\
\hline C3393-101 & 101.5 & $\mathrm{H} 2$ & $11.8 \%$ \\
\hline C3393-102.5 & 103.0 & $\mathrm{H} 2$ & $3.09 \%$ \\
\hline C3393-103.5 & 104.0 & $\mathrm{H} 2$ & $6.66 \%$ \\
\hline C3393-103.5 Dup & 104.0 & $\mathrm{H} 2$ & $7.38 \%$ \\
\hline C3393-105 & 105.5 & $\mathrm{H} 2$ & $5.83 \%$ \\
\hline C3393-106 & 106.5 & $\mathrm{H} 2$ & $15.2 \%$ \\
\hline C3393-107.5 & 108.0 & $\mathrm{H} 2$ & $6.76 \%$ \\
\hline C3393-108.5 & 109.0 & $\mathrm{H} 2$ & $8.80 \%$ \\
\hline C3393-108.5 Dup & 109.0 & $\mathrm{H} 2$ & $8.42 \%$ \\
\hline C3393-110 & 110.5 & $\mathrm{H} 2$ & $5.09 \%$ \\
\hline C3393-111 & 111.5 & $\mathrm{H} 2$ & $7.40 \%$ \\
\hline C3393-112.5 & 113.0 & $\mathrm{H} 2$ & $3.90 \%$ \\
\hline C3393-113.5 & 114.0 & $\mathrm{H} 2$ & $7.79 \%$ \\
\hline C3393-115 & 115.5 & $\mathrm{H} 2$ & $13.6 \%$ \\
\hline C3393-116 & 116.5 & $\mathrm{H} 2$ & $8.06 \%$ \\
\hline C3393-117.5 & 118.0 & $\mathrm{H} 2$ & $9.25 \%$ \\
\hline C3393-118.5 & 119.0 & $\mathrm{H} 2$ & $17.1 \%$ \\
\hline C3393-120 & 120.5 & $\mathrm{H} 2$ & $7.30 \%$ \\
\hline C3393-121 & 121.5 & $\mathrm{H} 2$ & $15.1 \%$ \\
\hline C3393-122.5 & 123.0 & $\mathrm{H} 2$ & $7.51 \%$ \\
\hline C3393-123.5 & 124.0 & $\mathrm{H} 2$ & $5.24 \%$ \\
\hline C3393-125 & 125.5 & $\mathrm{H} 2$ & $10.9 \%$ \\
\hline C3393-126 & 126.5 & $\mathrm{H} 2$ & $4.92 \%$ \\
\hline C3393-127.5 & 128.0 & $\mathrm{H} 2$ & $4.63 \%$ \\
\hline C3393-128.5 & 129.0 & $\mathrm{H} 2$ & $4.78 \%$ \\
\hline C3393-130 & 130.5 & $\mathrm{H} 2$ & $4.30 \%$ \\
\hline C3393-131 & 131.5 & $\mathrm{H} 2$ & $12.7 \%$ \\
\hline C3393-132.5 & 133.0 & $\mathrm{CCU}$ & $13.1 \%$ \\
\hline C3393-133.5 & 134.0 & $\mathrm{CCU}$ & $12.0 \%$ \\
\hline C3393-135 & 135.5 & $\mathrm{CCU}$ & $12.6 \%$ \\
\hline C3393-136 & 136.5 & $\mathrm{CCU}$ & $19.7 \%$ \\
\hline C3393-137.5 & 138.0 & $\mathrm{CCU}$ & $13.6 \%$ \\
\hline C3393-138.5 & 139.0 & $\mathrm{CCU}$ & $16.3 \%$ \\
\hline C3393-140 & 140.5 & $\mathrm{CCU}$ & $21.9 \%$ \\
\hline C3393-141 & 141.5 & $\mathrm{CCU}$ & $22.1 \%$ \\
\hline C3393-142.5 & 143.0 & $\mathrm{CCU}$ & $19.6 \%$ \\
\hline C3393-143.5 & 144.0 & $\mathrm{CCU}$ & $13.8 \%$ \\
\hline
\end{tabular}




\subsubsection{1:1 Sediment:Water Extracts of Sediments from Borehole C3393}

A subset of samples from the $\mathrm{C} 3393$ splitspoon cores were characterized by performing 1:1 sediment:water extracts. The following tables present the mass of a given constituent leached per gram of sediment as measured in the water extracts. Other figures show dilution-corrected values that represent concentrations in vadose zone pore water. As discussed in several other Vadose Zone Characterization Project reports, the dilution-corrected 1:1 sediment:water extracts are a reasonable estimate of the actual vadose zone pore water (Serne et al. 2002a, 2002b, 2002c, 2002d, 2002e, 2002f).

\subsubsection{1 pH and Electrical Conductivity}

The $\mathrm{pH}$ and electrical conductivity (EC) of the water extracts from select $\mathrm{C} 3393$ core samples are shown in Table 4.2. The $\mathrm{pH}$ is plotted as measured in the 1:1 sediment:water extracts, but the EC is corrected for dilution and plotted as if it was actual pore water. The $\mathrm{pH}$ profile is constant with all values between 7.2 and 8.0 (the typical range for Hanford sediments). The pore water-corrected EC data are slightly more variable, with a range of 0.978 to $3.56 \mathrm{mS} / \mathrm{cm}$ in the Hanford formation $\mathrm{H} 1$ unit and a range of 1.23 to $5.17 \mathrm{mS} / \mathrm{cm}$ in the Hanford formation $\mathrm{H} 2$ unit. The CCU had the largest variability in porewater corrected EC values, with a range of 1.25 to $10.6 \mathrm{mS} / \mathrm{cm}$. Overall, the calculated pore water conductivities were dilute and compared well with porewater conductivity data measured in other background boreholes (Serne et al. 2002a).

Table 4.2. $\mathrm{pH}$ for 1:1 Sediment:Water Extracts and Dilution-Corrected EC Values from Borehole C3393

\begin{tabular}{||l|c|c|c|c||}
\hline $\begin{array}{c}\text { Sample } \\
\text { ID }\end{array}$ & $\begin{array}{c}\text { Mid-Depth } \\
\mathrm{ft} \text { bgs }\end{array}$ & $\begin{array}{c}\text { Stratigraphic } \\
\text { Unit }\end{array}$ & $\mathrm{pH}$ & $\begin{array}{c}\text { Conductivity } \\
\text { (mS/cm) }\end{array}$ \\
\hline \hline C3393-6 & 6.5 & $\mathrm{H} 1$ & 7.25 & $9.78 \mathrm{E}-01$ \\
\hline C3393-16.5 & 17.0 & $\mathrm{H} 1$ & 7.19 & $3.56 \mathrm{E}+00$ \\
\hline C3393-19.25 & 19.0 & $\mathrm{H} 1$ & 7.28 & $2.76 \mathrm{E}+00$ \\
\hline C3393-51 & 51.5 & $\mathrm{H} 2$ & 7.21 & $4.87 \mathrm{E}+00$ \\
\hline C3393-57.5 & 58.0 & $\mathrm{H} 2$ & 7.29 & $1.50 \mathrm{E}+00$ \\
\hline C3393-71 & 71.5 & $\mathrm{H} 2$ & 7.19 & $5.17 \mathrm{E}+00$ \\
\hline C3393-76 & 76.5 & $\mathrm{H} 2$ & 7.46 & $1.65 \mathrm{E}+00$ \\
\hline C3393-83.5 & 84.0 & $\mathrm{H} 2$ & 7.40 & $2.61 \mathrm{E}+00$ \\
\hline C3393-86 & 86.5 & H2 & 7.41 & $1.61 \mathrm{E}+00$ \\
\hline C3393-91 & 91.5 & H2 & 7.48 & $1.48 \mathrm{E}+00$ \\
\hline C3393-96 & 96.5 & H2 & 7.42 & $3.09 \mathrm{E}+00$ \\
\hline C3393-103.5 & 104.0 & H2 & 7.55 & $3.09 \mathrm{E}+00$ \\
\hline C3393-103.5 Dup & 104.0 & H2 & 7.48 & $2.52 \mathrm{E}+00$ \\
\hline C3393-108.5 & 109.0 & H2 & 7.48 & $2.11 \mathrm{E}+00$ \\
\hline C3393-108.5 Dup & 109.0 & H2 & 7.52 & $2.25 \mathrm{E}+00$ \\
\hline C3393-118.5 & 119.0 & H2 & 7.51 & $1.23 \mathrm{E}+00$ \\
\hline C3393-125 & 125.5 & H2 & 7.67 & $1.78 \mathrm{E}+00$ \\
\hline C3393-126 & 126.5 & H2 & 7.78 & $2.93 \mathrm{E}+00$ \\
\hline C3393-136 & 136.5 & CCU & 8.00 & $1.24 \mathrm{E}+00$ \\
\hline C3393-141 & 141.5 & CCU & 7.76 & $1.06 \mathrm{E}+01$ \\
\hline EC values are dilution corrected and represent pore water concentrations not $1: 1$ extract values. \\
\hline
\end{tabular}




\subsubsection{Composition of the 1:1 Sediment:Water Extracts from the U Tank Farm Background Borehole}

The concentrations of major anions, cations, and several trace constituents are discussed in this section. The anion data are tabulated in Table 4.3 in units of mass per gram of dry sediment. A comparison of the masses of water-extractable anions per gram of sediment from the background sediments from the Hanford formation $\mathrm{H} 1$ and $\mathrm{H} 2$ units in $\mathrm{C} 3393$ showed that there is some variability in anion composition between the two formations. Namely, chloride concentrations were consistently higher in the Hanford formation $\mathrm{H} 2$ unit than in the $\mathrm{H} 1$ unit. This anomoly is not readily explainable, as the bulk composition of the sediments within the two formations is relatively similar. It is possible that a dilute waste stream containing small amounts of chloride could have traveled laterally along the interface between the two units and has slowly migrated deeper into the vadose zone (H2 unit) at this location. Other than small amounts of chloride, there are no other indications that material from this borehole should be excluded as representative of background conditions in the 241-U Tank Farm.

Table 4.3. Water-Extractable Anions in the U Tank Farm Background Borehole ( $\mu \mathrm{g} / \mathrm{g}$ dry sediment)

\begin{tabular}{|c|c|c|c|c|c|c|c|}
\hline $\begin{array}{l}\text { Sample } \\
\text { ID }\end{array}$ & $\begin{array}{l}\text { Mid-Depth } \\
\text { ft bgs }\end{array}$ & $\begin{array}{c}\text { Stratigraphic } \\
\text { Unit }\end{array}$ & $\begin{array}{c}\text { Fluoride } \\
\mu \mathrm{g} / \mathrm{g}\end{array}$ & $\begin{array}{c}\text { Chloride } \\
\mu \mathrm{g} / \mathrm{g}\end{array}$ & $\begin{array}{l}\text { Nitrate } \\
\mu \mathrm{g} / \mathrm{g}\end{array}$ & $\begin{array}{c}\text { Sulfate } \\
\mu \mathrm{g} / \mathrm{g}\end{array}$ & $\begin{array}{c}\text { Phosphate } \\
\mu \mathrm{g} / \mathrm{g}\end{array}$ \\
\hline C3393-6 & 6.5 & H1 & 9.33E-01 & $1.23 \mathrm{E}+00$ & $7.87 \mathrm{E}+00$ & $3.66 \mathrm{E}+00$ & $<2.40 \mathrm{E}-01$ \\
\hline C3393-16.5 & 17.0 & H1 & $4.98 \mathrm{E}-01$ & $2.76 \mathrm{E}-01$ & $1.44 \mathrm{E}+00$ & $5.97 \mathrm{E}+00$ & $<2.40 \mathrm{E}-01$ \\
\hline C3393-19.25 & 19.0 & $\mathrm{H} 1$ & $1.05 \mathrm{E}+00$ & $5.40 \mathrm{E}-01$ & $1.47 \mathrm{E}+00$ & $8.15 \mathrm{E}+00$ & $<2.41 \mathrm{E}-01$ \\
\hline C3393-51 & 51.5 & $\mathrm{H} 2$ & $3.46 \mathrm{E}-01$ & $6.89 \mathrm{E}+00$ & $1.70 \mathrm{E}+00$ & $3.20 \mathrm{E}+00$ & $<2.38 \mathrm{E}-01$ \\
\hline C3393-57.5 & 58.0 & $\mathrm{H} 2$ & $8.80 \mathrm{E}-01$ & $1.69 \mathrm{E}+01$ & $8.54 \mathrm{E}+00$ & $1.68 \mathrm{E}+01$ & $<2.40 \mathrm{E}-01$ \\
\hline C3393-71 & 71.5 & $\mathrm{H} 2$ & $6.85 \mathrm{E}-01$ & $6.46 \mathrm{E}+00$ & $3.32 \mathrm{E}+00$ & $1.88 \mathrm{E}+01$ & $<2.51 \mathrm{E}-01$ \\
\hline C3393-76 & 76.5 & $\mathrm{H} 2$ & $5.12 \mathrm{E}-01$ & $5.97 \mathrm{E}+00$ & $3.83 \mathrm{E}+00$ & $1.63 \mathrm{E}+01$ & $<2.40 \mathrm{E}-01$ \\
\hline C3393-83.5 & 84.0 & $\mathrm{H} 2$ & $5.79 \mathrm{E}-01$ & $5.31 \mathrm{E}+00$ & $1.57 \mathrm{E}+00$ & $1.01 \mathrm{E}+01$ & $<2.61 \mathrm{E}-01$ \\
\hline C3393-86 & 86.5 & $\mathrm{H} 2$ & $4.90 \mathrm{E}-02$ & $<7.32 \mathrm{E}-01$ & $2.92 \mathrm{E}-01$ & $1.59 \mathrm{E}+00$ & $<2.40 \mathrm{E}-01$ \\
\hline C3393-91 & 91.5 & $\mathrm{H} 2$ & $5.73 \mathrm{E}-01$ & $1.14 \mathrm{E}+01$ & $3.50 \mathrm{E}+00$ & $2.11 \mathrm{E}+01$ & $<2.40 \mathrm{E}-01$ \\
\hline C3393-96 & 96.5 & $\mathrm{H} 2$ & $<2.78 \mathrm{E}-02$ & $5.66 \mathrm{E}+01$ & $4.23 \mathrm{E}+00$ & $1.73 \mathrm{E}+01$ & $<2.38 \mathrm{E}-01$ \\
\hline C3393-103.5 & 104.0 & $\mathrm{H} 2$ & $4.65 \mathrm{E}-01$ & $4.71 \mathrm{E}+00$ & $3.21 \mathrm{E}+00$ & $1.47 \mathrm{E}+01$ & $<2.41 \mathrm{E}-01$ \\
\hline C3393-103.5 Dup & 104.0 & $\mathrm{H} 2$ & $5.44 \mathrm{E}-01$ & $7.40 \mathrm{E}+00$ & $1.63 \mathrm{E}+00$ & $8.47 \mathrm{E}+00$ & $3.03 \mathrm{E}-01$ \\
\hline C3393-108.5 & 109.0 & $\mathrm{H} 2$ & $5.14 \mathrm{E}-01$ & $7.62 \mathrm{E}+00$ & $1.63 \mathrm{E}+00$ & $8.13 \mathrm{E}+00$ & $4.92 \mathrm{E}-01$ \\
\hline C3393-108.5 Dup & 109.0 & $\mathrm{H} 2$ & $8.83 \mathrm{E}-01$ & $7.16 \mathrm{E}+00$ & $1.04 \mathrm{E}+00$ & $7.22 \mathrm{E}+00$ & $3.06 \mathrm{E}-01$ \\
\hline C3393-118.5 & 119.0 & $\mathrm{H} 2$ & 6.99E-01 & $3.89 \mathrm{E}+00$ & $9.05 \mathrm{E}-01$ & $5.33 \mathrm{E}+00$ & $<2.40 \mathrm{E}-01$ \\
\hline C3393-125 & 125.5 & $\mathrm{H} 2$ & $6.74 \mathrm{E}-01$ & $4.05 \mathrm{E}+00$ & $8.54 \mathrm{E}-01$ & $5.48 \mathrm{E}+00$ & $<2.40 \mathrm{E}-01$ \\
\hline C3393-126 & 126.5 & $\mathrm{H} 2$ & $5.13 \mathrm{E}-01$ & $1.85 \mathrm{E}+00$ & $6.10 \mathrm{E}-01$ & $4.40 \mathrm{E}+00$ & $<2.40 \mathrm{E}-01$ \\
\hline C3393-136 & 136.5 & $\mathrm{CCU}$ & $6.31 \mathrm{E}-01$ & $6.57 \mathrm{E}+00$ & 7.62E-01 & $1.17 \mathrm{E}+01$ & $4.38 \mathrm{E}-01$ \\
\hline C3393-141 & 141.5 & $\mathrm{CCU}$ & $7.55 \mathrm{E}-01$ & $6.42 \mathrm{E}+00$ & $9.31 \mathrm{E}-01$ & $8.48 \mathrm{E}+00$ & $5.73 \mathrm{E}-01$ \\
\hline
\end{tabular}

The water-extractable major cations in the U Tank Farm direct push sediments are tabulated in Table 4.4 in units of mass per gram of sediment on a dry weight basis. The majority of the samples analyzed from borehole C3393 contained more water-extractable sodium than calcium, including the calcium-rich CCU sediments. Results such as these generally indicate that the natural chemistry of the sediments has been altered by a sodium-based waste stream. Water-extractable sodium, although elevated, was not grossly elevated in comparison to calcium in the majority of these samples. This 
indicates that the waste stream impacting these sediments was likely more dilute with respect to sodium than typical tank waste streams.

Table 4.4. Water-Extractable Major Cations in the U Farm Background Borehole ( $\mu \mathrm{g} / \mathrm{g}$ dry sediment)

\begin{tabular}{|c|c|c|c|c|c|c|c|}
\hline $\begin{array}{l}\text { Sample } \\
\text { ID }\end{array}$ & $\begin{array}{l}\text { Mid- } \\
\text { Depth } \\
\text { ft bgs }\end{array}$ & $\begin{array}{c}\text { Stratigraphic } \\
\text { Unit }\end{array}$ & $\begin{array}{l}\text { Calcium } \\
\mu \mathrm{g} / \mathrm{g}\end{array}$ & $\begin{array}{l}\text { Potassium } \\
\mu \mathrm{g} / \mathrm{g}\end{array}$ & $\begin{array}{c}\text { Magnesium } \\
\mu \mathrm{g} / \mathrm{g}\end{array}$ & $\begin{array}{l}\text { Strontium } \\
\mu \mathrm{g} / \mathrm{g}\end{array}$ & $\begin{array}{l}\text { Sodium } \\
\mu \mathrm{g} / \mathrm{g}\end{array}$ \\
\hline C3393-6 & 6.5 & $\mathrm{H} 1$ & $1.00 \mathrm{E}+01$ & $8.47 \mathrm{E}-01$ & $8.73 \mathrm{E}+00$ & $1.17 \mathrm{E}-01$ & $1.34 \mathrm{E}+01$ \\
\hline C3393-16.5 & 17.0 & H1 & $8.78 \mathrm{E}+00$ & $3.47 \mathrm{E}+00$ & $1.54 \mathrm{E}+00$ & $4.06 \mathrm{E}-02$ & $1.51 \mathrm{E}+01$ \\
\hline C3393-19.25 & 19.0 & H1 & $1.13 \mathrm{E}+01$ & $4.20 \mathrm{E}+00$ & $2.06 \mathrm{E}+00$ & $5.40 \mathrm{E}-02$ & $1.60 \mathrm{E}+01$ \\
\hline C3393-51 & 51.5 & $\mathrm{H} 2$ & $7.50 \mathrm{E}+00$ & $4.04 \mathrm{E}+00$ & $2.99 \mathrm{E}+00$ & $5.49 \mathrm{E}-02$ & $8.14 E+00$ \\
\hline C3393-57.5 & 58.0 & $\mathrm{H} 2$ & $1.44 \mathrm{E}+01$ & $5.44 \mathrm{E}+00$ & $6.03 \mathrm{E}+00$ & $9.50 \mathrm{E}-02$ & $1.22 \mathrm{E}+01$ \\
\hline C3393-71 & 71.5 & $\mathrm{H} 2$ & $6.51 \mathrm{E}+00$ & $3.42 \mathrm{E}+00$ & $2.17 \mathrm{E}+00$ & $4.10 \mathrm{E}-02$ & $9.16 E+00$ \\
\hline C3393-76 & 76.5 & $\mathrm{H} 2$ & $1.05 \mathrm{E}+01$ & $4.95 \mathrm{E}+00$ & $4.06 \mathrm{E}+00$ & $6.77 \mathrm{E}-02$ & $1.69 \mathrm{E}+01$ \\
\hline C3393-83.5 & 84.0 & $\mathrm{H} 2$ & $1.02 \mathrm{E}+01$ & $4.25 \mathrm{E}+00$ & $3.01 \mathrm{E}+00$ & $5.50 \mathrm{E}-02$ & $1.65 E+01$ \\
\hline C3393-86 & 86.5 & $\mathrm{H} 2$ & $1.21 \mathrm{E}+01$ & $3.71 \mathrm{E}+00$ & $2.99 \mathrm{E}+00$ & $6.77 \mathrm{E}-02$ & $1.46 \mathrm{E}+01$ \\
\hline C3393-91 & 91.5 & $\mathrm{H} 2$ & $1.42 \mathrm{E}+01$ & $4.55 \mathrm{E}+00$ & $4.60 \mathrm{E}+00$ & $7.58 \mathrm{E}-02$ & $1.86 \mathrm{E}+01$ \\
\hline C3393-96 & 96.5 & $\mathrm{H} 2$ & $2.73 \mathrm{E}+01$ & $4.70 \mathrm{E}+00$ & $6.31 \mathrm{E}+00$ & $1.40 \mathrm{E}-01$ & $1.72 \mathrm{E}+01$ \\
\hline C3393-103.5 & 104.0 & $\mathrm{H} 2$ & $1.02 \mathrm{E}+01$ & $4.85 \mathrm{E}+00$ & $2.48 \mathrm{E}+00$ & $5.46 \mathrm{E}-02$ & $2.02 E+01$ \\
\hline C3393-103.5 Dup & 104.0 & $\mathrm{H} 2$ & $1.02 \mathrm{E}+01$ & $3.97 \mathrm{E}+00$ & $2.34 \mathrm{E}+00$ & $5.36 \mathrm{E}-02$ & $1.68 \mathrm{E}+01$ \\
\hline C3393-108.5 & 109.0 & $\mathrm{H} 2$ & $7.97 \mathrm{E}+00$ & $2.52 \mathrm{E}+00$ & $1.78 \mathrm{E}+00$ & $3.84 \mathrm{E}-02$ & $2.27 \mathrm{E}+01$ \\
\hline C3393-108.5 Dup & 109.0 & $\mathrm{H} 2$ & $8.17 \mathrm{E}+00$ & $2.58 \mathrm{E}+00$ & $1.82 \mathrm{E}+00$ & $3.89 \mathrm{E}-02$ & $2.25 E+01$ \\
\hline C3393-118.5 & 119.0 & $\mathrm{H} 2$ & $7.55 \mathrm{E}+00$ & $3.03 \mathrm{E}+00$ & $1.75 \mathrm{E}+00$ & $3.72 \mathrm{E}-02$ & $2.80 \mathrm{E}+01$ \\
\hline C3393-125 & 125.5 & $\mathrm{H} 2$ & $8.18 \mathrm{E}+00$ & $3.89 \mathrm{E}+00$ & $2.00 \mathrm{E}+00$ & $4.08 \mathrm{E}-02$ & $2.46 \mathrm{E}+01$ \\
\hline C3393-126 & 126.5 & $\mathrm{H} 2$ & $6.46 \mathrm{E}+00$ & $3.05 \mathrm{E}+00$ & $1.49 \mathrm{E}+00$ & $3.47 \mathrm{E}-02$ & $1.81 \mathrm{E}+01$ \\
\hline C3393-136 & 136.5 & $\mathrm{CCU}$ & $8.96 \mathrm{E}+00$ & $3.23 \mathrm{E}+00$ & $2.65 \mathrm{E}+00$ & $4.37 \mathrm{E}-02$ & $3.37 \mathrm{E}+01$ \\
\hline C3393-141 & 141.5 & $\mathrm{CCU}$ & $9.81 \mathrm{E}+00$ & $2.95 \mathrm{E}+00$ & $3.72 \mathrm{E}+00$ & $5.01 \mathrm{E}-02$ & $2.76 \mathrm{E}+01$ \\
\hline
\end{tabular}

The water-extractable aluminum, iron, sulfur, and phosphorus in the U Farm background borehole sediments are shown in Table 4.5. The sulfur and phosphorus data were converted to water-extractable sulfur as sulfate and phosphorus as phosphate so that the results could be compared to the IC data presented in Table 4.3. The agreement between directly measured sulfate in the water extracts using ion chromatography and indirectly by converting the ICP measurements for sulfur to sulfate was not very good. Differences ranging from $13 \%$ to as much as $168 \%$ were calculated between the two data sets. In most cases, the sulfate data generated directly by IC analysis were higher than the sulfate converted via analysis by ICP-OES, indicating that the ICP-OES data was likely biased low. Comparison of phosphate directly measured via IC and ICP-OES phosphorus data converted to phosphate resulted in slightly better agreement between the two data sets. Percent differences for the data sets ranged from approximately $10 \%$ to $57 \%$. However, in this case, the ICP-OES-derived data were generally higher than the IC data. It is likely that agreement between the two analytical techniques was less than optimal given the low phosphate content of the samples. Water-soluble iron was below quantifiable levels in all but one of the background borehole samples analyzed. Water-soluble aluminum was below quantifiable levels in all of the background borehole samples analyzed.

The water extract data for potentially mobile metals, such as technetium-99, uranium-238, chromium, and molybdenum are shown in Table 4.6. None of the samples tested contained quantifiable waterextractable concentrations of technetium-99, and few of the samples contained quantifiable concentrations of water-extractable chromium. All but three of the samples contained trace amounts of water-extractable 
molybdenum, and all of the samples tested contained trace amounts of water-extractable uranium. The lack of quantifiable concentrations of water-extractable technetium and chromium, combined with the presence of only trace water-extractable uranium, indicates that the waste source impacting the vadose zone at this location was at most, distantly associated with tank waste-producing processes.

Table 4.5. Water-Extractable Cations in the C3393 Borehole Core Samples ( $\mu \mathrm{g} / \mathrm{g}$ dry sediment)

\begin{tabular}{||l|c|c|c|c|c|c||}
\hline $\begin{array}{c}\text { Sample } \\
\text { ID }\end{array}$ & $\begin{array}{c}\text { Mid-Depth } \\
\mathrm{ft} \text { bgs }\end{array}$ & $\begin{array}{c}\text { Stratigraphic } \\
\text { Unit }\end{array}$ & $\begin{array}{c}\text { Aluminum } \\
\mu \mathrm{g} / \mathrm{g}\end{array}$ & $\begin{array}{c}\text { Iron } \\
\mu \mathrm{g} / \mathrm{g}\end{array}$ & $\begin{array}{c}\text { Sulfur as } \mathrm{SO}_{4}{ }^{2-} \\
\mu \mathrm{g} / \mathrm{g}\end{array}$ & $\begin{array}{c}\text { Phosphorus } \\
\text { as PO}_{4}{ }^{3-} \\
\mu \mathrm{g} / \mathrm{g}\end{array}$ \\
\hline \hline C3393-6 & 6.5 & $\mathrm{H} 1$ & $(8.86 \mathrm{E}-03)$ & $(1.77 \mathrm{E}-02)$ & $4.67 \mathrm{E}+00$ & $(4.11 \mathrm{E}-02)$ \\
\hline C3393-16.5 & 17.0 & $\mathrm{H} 1$ & $(3.61 \mathrm{E}-02)$ & $(8.42 \mathrm{E}-02)$ & $6.87 \mathrm{E}+00$ & $1.74 \mathrm{E}-01$ \\
\hline C3393-19.25 & 19.0 & $\mathrm{H} 1$ & $(4.30 \mathrm{E}-02)$ & $(9.17 \mathrm{E}-02)$ & $1.07 \mathrm{E}+01$ & $1.74 \mathrm{E}-01$ \\
\hline C3393-51 & 51.5 & $\mathrm{H} 2$ & $(8.24 \mathrm{E}-02)$ & $(4.37 \mathrm{E}-02)$ & $4.29 \mathrm{E}+00$ & $8.58 \mathrm{E}-02$ \\
\hline C3393-57.5 & 58.0 & $\mathrm{H} 2$ & $(2.95 \mathrm{E}-03)$ & $(1.69 \mathrm{E}-02)$ & $1.36 \mathrm{E}+01$ & $1.48 \mathrm{E}-01$ \\
\hline C3393-71 & 71.5 & $\mathrm{H} 2$ & $(2.93 \mathrm{E}-02)$ & $(2.26 \mathrm{E}-02)$ & $7.38 \mathrm{E}+00$ & $1.08 \mathrm{E}-01$ \\
\hline C3393-76 & 76.5 & $\mathrm{H} 2$ & $(1.72 \mathrm{E}-02)$ & $(7.25 \mathrm{E}-02)$ & $1.98 \mathrm{E}+01$ & $1.67 \mathrm{E}-01$ \\
\hline C3393-83.5 & 84.0 & $\mathrm{H} 2$ & $(3.12 \mathrm{E}-02)$ & $(6.89 \mathrm{E}-02)$ & $1.24 \mathrm{E}+01$ & $2.19 \mathrm{E}-01$ \\
\hline C3393-86 & 86.5 & $\mathrm{H} 2$ & $(1.30 \mathrm{E}-02)$ & $(3.88 \mathrm{E}-02)$ & $1.83 \mathrm{E}+01$ & $1.94 \mathrm{E}-01$ \\
\hline C3393-91 & 91.5 & $\mathrm{H} 2$ & $(3.25 \mathrm{E}-03)$ & $(3.46 \mathrm{E}-02)$ & $2.49 \mathrm{E}+01$ & $2.18 \mathrm{E}-01$ \\
\hline C3393-96 & 96.5 & $\mathrm{H} 2$ & $<4.96 \mathrm{E}-01$ & $(1.06 \mathrm{E}-02)$ & $2.08 \mathrm{E}+01$ & $2.44 \mathrm{E}-01$ \\
\hline C3393-103.5 & 104.0 & $\mathrm{H} 2$ & $(2.51 \mathrm{E}-02)$ & $(2.15 \mathrm{E}-02)$ & $2.15 \mathrm{E}+01$ & $1.59 \mathrm{E}-01$ \\
\hline C3393-103.5 Dup & 104.0 & $\mathrm{H} 2$ & $(2.39 \mathrm{E}-02)$ & $(2.99 \mathrm{E}-02)$ & $1.79 \mathrm{E}+01$ & $2.06 \mathrm{E}-01$ \\
\hline C3393-108.5 & 109.0 & $\mathrm{H} 2$ & $(2.21 \mathrm{E}-03)$ & $(4.80 \mathrm{E}-02)$ & $1.02 \mathrm{E}+01$ & $4.16 \mathrm{E}-01$ \\
\hline C3393-108.5 Dup & 109.0 & $\mathrm{H} 2$ & $(1.07 \mathrm{E}-02)$ & $(7.05 \mathrm{E}-02)$ & $1.02 \mathrm{E}+01$ & $4.31 \mathrm{E}-01$ \\
\hline C3393-118.5 & 119.0 & $\mathrm{H} 2$ & $(9.10 \mathrm{E}-03)$ & $1.54 \mathrm{E}-01$ & $8.83 \mathrm{E}+00$ & $4.58 \mathrm{E}-01$ \\
\hline C3393-125 & 125.5 & $\mathrm{H} 2$ & $(3.53 \mathrm{E}-03)$ & $(3.27 \mathrm{E}-02)$ & $6.97 \mathrm{E}+00$ & $3.00 \mathrm{E}-01$ \\
\hline C3393-126 & 126.5 & $\mathrm{H} 2$ & $(2.45 \mathrm{E}-02)$ & $(6.65 \mathrm{E}-02)$ & $5.46 \mathrm{E}+00$ & $2.42 \mathrm{E}-01$ \\
\hline C3393-136 & 136.5 & $\mathrm{CCU}$ & $<5.01 \mathrm{E}-01$ & $(7.26 \mathrm{E}-02)$ & $1.03 \mathrm{E}+01$ & $7.85 \mathrm{E}-01$ \\
\hline C3393-141 & 141.5 & $\mathrm{CCU}$ & $<5.02 \mathrm{E}-01$ & $(9.48 \mathrm{E}-03)$ & $1.39 \mathrm{E}+01$ & $6.32 \mathrm{E}-01$ \\
\hline $\begin{array}{l}\text { Parentheses indicate reported value was less than the limit of quantification for the analysis. } \\
\text { Less-than values indicate } \\
\text { reported. }\end{array}$ & & & & \\
\hline
\end{tabular}

Table 4.6. Water-Extractable Mobile Metals in the C3393 Borehole Core Samples ( $\mu \mathrm{g} / \mathrm{g}$ dry sediment)

\begin{tabular}{||l|c|c|c|c|c|c||}
\hline $\begin{array}{c}\text { Sample } \\
\text { ID }\end{array}$ & $\begin{array}{c}\text { Mid-Depth } \\
\mathrm{ft} \text { bgs }\end{array}$ & $\begin{array}{c}\text { Stratigraphic } \\
\text { Unit }\end{array}$ & $\begin{array}{c}\text { Technetium- } \\
99(\mathrm{pCi} / \mathrm{g})\end{array}$ & $\begin{array}{c}\text { Uranium } \\
(\mu \mathrm{g} / \mathrm{g})\end{array}$ & $\begin{array}{c}\text { Chromium-53 } \\
(\mu \mathrm{g} / \mathrm{g})\end{array}$ & $\begin{array}{c}\text { Molybdenum-||} \\
95(\mu \mathrm{g} / \mathrm{g})\end{array}$ \\
\hline \hline C3393-6 & 6.5 & $\mathrm{H} 1$ & $(2.54 \mathrm{E}-02)$ & $1.54 \mathrm{E}-03$ & $(1.00 \mathrm{E}-03)$ & $(2.35 \mathrm{E}-03)$ \\
\hline C3393-16.5 & 17.0 & $\mathrm{H} 1$ & $(3.39 \mathrm{E}-02)$ & $1.79 \mathrm{E}-03$ & $(1.35 \mathrm{E}-04)$ & $(1.77 \mathrm{E}-03)$ \\
\hline C3393-19.25 & 19.0 & H1 & $(3.41 \mathrm{E}-02)$ & $1.25 \mathrm{E}-03$ & $(3.82 \mathrm{E}-03)$ & $3.95 \mathrm{E}-03$ \\
\hline C3393-51 & 51.5 & H2 & $(4.21 \mathrm{E}-02)$ & $1.93 \mathrm{E}-04$ & $1.70 \mathrm{E}-02$ & $1.23 \mathrm{E}-02$ \\
\hline C3393-57.5 & 58.0 & H2 & $(4.24 \mathrm{E}-02)$ & $1.03 \mathrm{E}-03$ & $(1.34 \mathrm{E}-03)$ & $5.64 \mathrm{E}-03$ \\
\hline C3393-71 & 71.5 & H2 & $(2.66 \mathrm{E}-02)$ & $2.69 \mathrm{E}-04$ & $(8.21 \mathrm{E}-04)$ & $4.74 \mathrm{E}-03$ \\
\hline C3393-76 & 76.5 & H2 & $(4.24 \mathrm{E}-02)$ & $8.05 \mathrm{E}-04$ & $(1.22 \mathrm{E}-03)$ & $1.50 \mathrm{E}-02$ \\
\hline C3393-83.5 & 84.0 & H2 & $(4.61 \mathrm{E}-02)$ & $5.02 \mathrm{E}-04$ & $(7.22 \mathrm{E}-04)$ & $1.53 \mathrm{E}-02$ \\
\hline C3393-86 & 86.5 & H2 & $(4.25 \mathrm{E}-02)$ & $6.78 \mathrm{E}-04$ & $4.60 \mathrm{E}-02$ & $1.99 \mathrm{E}-02$ \\
\hline C3393-91 & 91.5 & H2 & $(3.39 \mathrm{E}-02)$ & $1.13 \mathrm{E}-03$ & $5.09 \mathrm{E}-03$ & $1.23 \mathrm{E}-02$ \\
\hline C33393-96 & 96.5 & H2 & $(5.89 \mathrm{E}-02)$ & $6.05 \mathrm{E}-04$ & $(2.80 \mathrm{E}-03)$ & $1.05 \mathrm{E}-02$ \\
\hline C3393-103.5 & 104.0 & H2 & $(3.41 \mathrm{E}-02)$ & $4.42 \mathrm{E}-04$ & $(4.47 \mathrm{E}-03)$ & $3.41 \mathrm{E}-02$ \\
\hline C3333-103.5 Dup & 104.0 & H2 & $(3.47 \mathrm{E}-02)$ & $4.57 \mathrm{E}-04$ & $(2.37 \mathrm{E}-03)$ & $2.32 \mathrm{E}-02$ \\
\hline C3393-108.5 & 109.0 & H2 & $(4.25 \mathrm{E}-02)$ & $7.56 \mathrm{E}-04$ & $(1.02 \mathrm{E}-03)$ & $8.94 \mathrm{E}-03$ \\
\hline
\end{tabular}




\begin{tabular}{|l|c|c|c|c|c|c||}
\hline C3393-108.5 Dup & 109.0 & $\mathrm{H} 2$ & $(2.57 \mathrm{E}-02)$ & $7.57 \mathrm{E}-04$ & $(2.60 \mathrm{E}-03)$ & $9.74 \mathrm{E}-03$ \\
\hline C3393-118.5 & 119.0 & $\mathrm{H} 2$ & $(3.39 \mathrm{E}-02)$ & $8.88 \mathrm{E}-04$ & $(1.20 \mathrm{E}-03)$ & $1.53 \mathrm{E}-02$ \\
\hline C3393-125 & 125.5 & $\mathrm{H} 2$ & $(3.40 \mathrm{E}-02)$ & $1.28 \mathrm{E}-03$ & $5.77 \mathrm{E}-03$ & $8.16 \mathrm{E}-03$ \\
\hline C3393-126 & 126.5 & $\mathrm{H} 2$ & $(3.40 \mathrm{E}-02)$ & $9.82 \mathrm{E}-04$ & $(2.83 \mathrm{E}-04)$ & $(1.68 \mathrm{E}-03)$ \\
\hline C3393-136 & 136.5 & $\mathrm{CCU}$ & $(4.25 \mathrm{E}-02)$ & $1.76 \mathrm{E}-03$ & $(3.73 \mathrm{E}-03)$ & $4.83 \mathrm{E}-03$ \\
\hline C3393-141 & 141.5 & $\mathrm{CCU}$ & $(3.41 \mathrm{E}-02)$ & $1.62 \mathrm{E}-03$ & $6.06 \mathrm{E}-02$ & $4.15 \mathrm{E}-02$ \\
\hline
\end{tabular}

\subsubsection{Vadose Zone Porewater Chemical Composition in the Background Borehole}

The 1:1 water extract data was converted to derive the pore water composition of the vadose zone sediments so that electrical balances (anions vs. cation) of the samples could be performed. From knowledge of the moisture content of the sediment samples taken from the liners of each direct push sampler, the amount of de-ionized water that would be needed to make the water extract exactly one part water (total of native pore water and added de-ionized water) to one part by weight dry sediment was calculated. The ratio of the total volume of water in the extract to the native mass of pore water is the dilution factor. An assumption was made that the de-ionized water acted solely as a diluent of the existing pore water and that the de-ionized water did not dissolve any of the solids in the sediments. Thus, by correcting for the dilution, an estimate of the actual chemical composition of the native porewater in the vadose zone sediments could be derived.

The assumption that none of the solid is dissolved during the water extraction process is simplistic. In comparisons of actual vadose zone sediment pore water, which was obtained via ultracentrifugation of sediments, to the dilution-corrected calculated pore waters from both contaminated and uncontaminated sediments from the SX and B-BX Tank Farms (see Serne et al. 2002b, 2002c, 2002d, 2002e, 2002f), it was found that for highly contaminated sediments, the comparison is quite good. For slightly contaminated or uncontaminated sediments, the dilution-corrected water extract data is biased high by a factor of 2 to $7 \mathrm{x}$ for many constituents such that the true pore water is less saline.

Tables 4.7 and 4.8 show the derived pore water composition of key constituents in meq/L. The majority of the background borehole samples contained relatively low dissolved salt loads, with values that ranged from a low of $18 \mathrm{meq} / \mathrm{L}$ total (anions and cations) for samples collected from 6 and $25 \mathrm{ft} \mathrm{bgs,}$ to a high of $105 \mathrm{meq} / \mathrm{L}$ for the sample collected from $71 \mathrm{ft}$ bgs. The most saline sample analyzed, C339371, contained $17.0 \mathrm{meq} / \mathrm{L}$ sodium, $13.8 \mathrm{meq} / \mathrm{L}$ calcium, $7.61 \mathrm{meq} / \mathrm{L}$ magnesium, and $3.72 \mathrm{meq} / \mathrm{L}$ potassium. The dissolved cations in this sample were primarily compensated by bicarbonate $(34.7$ $\mathrm{meq} / \mathrm{L})$, with lesser amounts of sulfate $(16.6 \mathrm{meq} / \mathrm{L})$, chloride $(7.77 \mathrm{meq} / \mathrm{L})$, and nitrate $(2.28 \mathrm{meq} / \mathrm{L})$. The porewater calculated concentrations of key contaminants of concern are presented in Table 4.9. None of the mobile metals reported in Table 4.9 were found at elevated activities or concentrations in the background sediment samples.

All twenty of the samples analyzed contained bicarbonate as the dominant water-extractable anion. All but three of the background sediment samples tested contained sodium as the dominant waterextractable cation. The divalent cations calcium and magnesium are the dominant exchangeable cations in most Hanford sediments. Therefore, samples that contain a different dominant exchangeable cation generally indicate that the sediment properties have been impacted by a waste stream. In the case of the background borehole, samples beginning at approximately $110 \mathrm{ft}$ bgs contained 2.5 to over 3 times more water-extractable sodium than calcium. This trend suggests that waste fluids that were high in sodium 
have impacted the vadose zone near this borehole. The source appears to be a relatively dilute sodiumbearing waste solution. The sodium from this source has pushed some to most of the natural divalent cations off the sediment cation exchange sites through the deepest sample collected as part of this campaign. As a result, the maximum depth of the cation exchange front is unknown at this time.

Overall, the calculated charge balance between cations and anions for all of the samples was quite good (less than 10\% difference for most of the samples analyzed). However, samples C3393-71, C339386, and C3393-96 had charge differences of $-37.6,+37.6$, and +18.2 , respectively. Sample C3393-71 contained more dissolved anions than cations, while the other two samples contained more cations than anions. The most likely source of the discrepancy between these samples was in the measurement of bicarbonate via titration with acid.

Table 4.7. Calculated Pore Water Anion Concentrations in the C3393 Borehole Core Samples

\begin{tabular}{|c|c|c|c|c|c|c|c|c|}
\hline $\begin{array}{l}\text { Sample } \\
\text { ID }\end{array}$ & $\begin{array}{l}\text { Mid- } \\
\text { Depth } \\
\text { ft bgs }\end{array}$ & $\begin{array}{c}\text { Stratigraphic } \\
\text { Unit }\end{array}$ & $\begin{array}{c}\text { Fluoride } \\
\text { meq/L }\end{array}$ & $\begin{array}{l}\text { Chloride } \\
\text { meq/L }\end{array}$ & $\begin{array}{l}\text { Nitrate } \\
\mathrm{meq} / \mathrm{L}\end{array}$ & $\begin{array}{l}\text { Sulfate } \\
\mathrm{meq} / \mathrm{L}\end{array}$ & $\begin{array}{c}\text { Phosphate } \\
\text { meq/L }\end{array}$ & $\begin{array}{c}\text { Alkalinity } \\
\text { meq/L }\end{array}$ \\
\hline C3393-6 & 6.5 & $\mathrm{H} 1$ & $2.42 \mathrm{E}-01$ & $1.70 \mathrm{E}-01$ & $6.24 \mathrm{E}-01$ & $3.75 \mathrm{E}-01$ & $<3.73 \mathrm{E}-02$ & $8.28 \mathrm{E}+00$ \\
\hline C3393-16.5 & 17.0 & $\mathrm{H} 1$ & $6.34 \mathrm{E}-01$ & $1.88 \mathrm{E}-01$ & $5.62 \mathrm{E}-01$ & $3.01 \mathrm{E}+00$ & $<1.83 \mathrm{E}-01$ & $2.90 \mathrm{E}+01$ \\
\hline C3393-19.25 & 19.0 & $\mathrm{H} 1$ & $8.79 \mathrm{E}-01$ & $2.42 \mathrm{E}-01$ & $3.76 \mathrm{E}-01$ & $2.69 \mathrm{E}+00$ & $<1.21 \mathrm{E}-01$ & $2.26 \mathrm{E}+01$ \\
\hline C3393-51 & 51.5 & $\mathrm{H} 2$ & $6.86 \mathrm{E}-01$ & $7.33 \mathrm{E}+00$ & $1.03 \mathrm{E}+00$ & $2.51 \mathrm{E}+00$ & $<2.84 \mathrm{E}-01$ & $3.27 \mathrm{E}+01$ \\
\hline \begin{tabular}{|l|} 
C3393-57.5 \\
\end{tabular} & 58.0 & $\mathrm{H} 2$ & $3.16 \mathrm{E}-01$ & $3.24 \mathrm{E}+00$ & $9.38 \mathrm{E}-01$ & $2.38 \mathrm{E}+00$ & $<5.17 \mathrm{E}-02$ & $6.70 \mathrm{E}+00$ \\
\hline C3393-71 & 71.5 & $\mathrm{H} 2$ & $1.54 \mathrm{E}+00$ & $7.77 \mathrm{E}+00$ & $2.28 \mathrm{E}+00$ & $1.66 \mathrm{E}+01$ & $<3.38 \mathrm{E}-01$ & $3.47 \mathrm{E}+01$ \\
\hline C3393-76 & 76.5 & $\mathrm{H} 2$ & $2.27 \mathrm{E}-01$ & $1.42 \mathrm{E}+00$ & $5.20 \mathrm{E}-01$ & $2.85 \mathrm{E}+00$ & $<6.38 \mathrm{E}-02$ & $9.71 \mathrm{E}+00$ \\
\hline C3393-83.5 & 84.0 & $\mathrm{H} 2$ & $4.27 \mathrm{E}-01$ & $2.10 \mathrm{E}+00$ & $3.56 \mathrm{E}-01$ & $2.95 \mathrm{E}+00$ & $<1.16 \mathrm{E}-01$ & $1.62 \mathrm{E}+01$ \\
\hline C3393-86 & 86.5 & $\mathrm{H} 2$ & $2.22 \mathrm{E}-02$ & $<1.77 \mathrm{E}-01$ & $4.05 \mathrm{E}-02$ & $2.84 \mathrm{E}-01$ & $<6.53 \mathrm{E}-02$ & $8.72 \mathrm{E}+00$ \\
\hline C3393-91 & 91.5 & $\mathrm{H} 2$ & $1.90 \mathrm{E}-01$ & $2.02 \mathrm{E}+00$ & $3.56 \mathrm{E}-01$ & $2.77 \mathrm{E}+00$ & $<4.78 \mathrm{E}-02$ & $7.76 \mathrm{E}+00$ \\
\hline C3393-96 & 96.5 & $\mathrm{H} 2$ & $<1.36 \mathrm{E}-02$ & $1.48 \mathrm{E}+01$ & $6.32 \mathrm{E}-01$ & $3.33 \mathrm{E}+00$ & $<6.98 \mathrm{E}-02$ & $2.44 \mathrm{E}+00$ \\
\hline C3393-103.5 & 104.0 & $\mathrm{H} 2$ & $3.67 \mathrm{E}-01$ & $1.99 \mathrm{E}+00$ & $7.78 \mathrm{E}-01$ & $4.59 \mathrm{E}+00$ & $<1.14 \mathrm{E}-01$ & $1.60 \mathrm{E}+01$ \\
\hline C3393-103.5 Dup & 104.0 & $\mathrm{H} 2$ & $3.87 \mathrm{E}-01$ & $2.83 \mathrm{E}+00$ & $3.55 \mathrm{E}-01$ & $2.39 \mathrm{E}+00$ & $1.30 \mathrm{E}-01$ & $1.49 \mathrm{E}+01$ \\
\hline C3393-108.5 & 109.0 & $\mathrm{H} 2$ & $3.07 \mathrm{E}-01$ & $2.44 \mathrm{E}+00$ & $2.98 \mathrm{E}-01$ & $1.92 \mathrm{E}+00$ & $1.76 \mathrm{E}-01$ & $1.30 \mathrm{E}+01$ \\
\hline C3393-108.5 Dup & 109.0 & $\mathrm{H} 2$ & $5.52 \mathrm{E}-01$ & $2.40 \mathrm{E}+00$ & $1.99 \mathrm{E}-01$ & $1.79 \mathrm{E}+00$ & $1.15 \mathrm{E}-01$ & $1.44 \mathrm{E}+01$ \\
\hline C3393-118.5 & 119.0 & $\mathrm{H} 2$ & $2.15 \mathrm{E}-01$ & $6.42 \mathrm{E}-01$ & $8.53 \mathrm{E}-02$ & $6.49 \mathrm{E}-01$ & $<4.43 \mathrm{E}-02$ & $9.21 \mathrm{E}+00$ \\
\hline C3393-125 & 125.5 & $\mathrm{H} 2$ & $3.25 \mathrm{E}-01$ & $1.05 \mathrm{E}+00$ & $1.26 \mathrm{E}-01$ & $1.05 \mathrm{E}+00$ & $<6.95 \mathrm{E}-02$ & ND \\
\hline C3393-126 & 126.5 & $\mathrm{H} 2$ & 5.49E-01 & $1.06 \mathrm{E}+00$ & $2.00 \mathrm{E}-01$ & $1.86 \mathrm{E}+00$ & $<1.54 \mathrm{E}-01$ & $2.38 \mathrm{E}+01$ \\
\hline C3393-136 & 136.5 & $\mathrm{CCU}$ & $1.69 \mathrm{E}-01$ & $9.42 \mathrm{E}-01$ & $6.25 \mathrm{E}-02$ & $1.24 \mathrm{E}+00$ & $7.03 \mathrm{E}-02$ & $9.28 \mathrm{E}+00$ \\
\hline C3393-141 & 141.5 & $\mathrm{CCU}$ & $1.80 \mathrm{E}-01$ & $8.21 \mathrm{E}-01$ & $6.80 \mathrm{E}-02$ & $8.00 \mathrm{E}-01$ & $8.21 \mathrm{E}-02$ & $7.23 \mathrm{E}+00$ \\
\hline
\end{tabular}

Table 4.8. Calculated Pore Water Cation Concentrations in the C3393 Borehole Core Samples

\begin{tabular}{||l|c|c|c|c|c|c||}
\hline $\begin{array}{c}\text { Sample } \\
\text { ID }\end{array}$ & $\begin{array}{c}\text { Mid-Depth } \\
\mathrm{ft} \text { bgs }\end{array}$ & $\begin{array}{c}\text { Stratigraphic } \\
\text { Unit }\end{array}$ & $\begin{array}{c}\text { Calcium } \\
\text { meq/L }\end{array}$ & $\begin{array}{c}\text { Potassium } \\
\text { meq/L }\end{array}$ & $\begin{array}{c}\text { Magnesium } \\
\text { meq/L }\end{array}$ & $\begin{array}{c}\text { Sodium } \\
\text { meq/L }\end{array}$ \\
\hline C3393-6 & 6.5 & H1 & $2.46 \mathrm{E}+00$ & $1.07 \mathrm{E}-01$ & $3.53 \mathrm{E}+00$ & $2.86 \mathrm{E}+00$ \\
\hline C3393-16.5 & 17.0 & H1 & $1.06 \mathrm{E}+01$ & $2.15 \mathrm{E}+00$ & $3.06 \mathrm{E}+00$ & $1.59 \mathrm{E}+01$ \\
\hline C3393-19.25 & 19.0 & H1 & $8.94 \mathrm{E}+00$ & $1.70 \mathrm{E}+00$ & $2.68 \mathrm{E}+00$ & $1.10 \mathrm{E}+01$ \\
\hline C3393-51 & 51.5 & H2 & $1.41 \mathrm{E}+01$ & $3.90 \mathrm{E}+00$ & $9.28 \mathrm{E}+00$ & $1.34 \mathrm{E}+01$ \\
\hline
\end{tabular}




\begin{tabular}{||l|c|c|c|c|c|c||}
\hline $\begin{array}{c}\text { Sample } \\
\text { ID }\end{array}$ & $\begin{array}{c}\text { Mid-Depth } \\
\text { ft bgs }\end{array}$ & $\begin{array}{c}\text { Stratigraphic } \\
\text { Unit }\end{array}$ & $\begin{array}{c}\text { Calcium } \\
\text { meq/L }\end{array}$ & $\begin{array}{c}\text { Potassium } \\
\text { meq/L }\end{array}$ & $\begin{array}{c}\text { Magnesium } \\
\text { meq/L }\end{array}$ & $\begin{array}{c}\text { Sodium } \\
\text { meq/L }\end{array}$ \\
\hline C3393-57.5 & 58.0 & H2 & $4.90 \mathrm{E}+00$ & $9.49 \mathrm{E}-01$ & $3.38 \mathrm{E}+00$ & $3.61 \mathrm{E}+00$ \\
\hline C3393-71 & 71.5 & H2 & $1.38 \mathrm{E}+01$ & $3.72 \mathrm{E}+00$ & $7.61 \mathrm{E}+00$ & $1.70 \mathrm{E}+01$ \\
\hline C3393-76 & 76.5 & H2 & $4.39 \mathrm{E}+00$ & $1.07 \mathrm{E}+00$ & $2.81 \mathrm{E}+00$ & $6.17 \mathrm{E}+00$ \\
\hline C3393-83.5 & 84.0 & H2 & $7.17 \mathrm{E}+00$ & $1.53 \mathrm{E}+00$ & $3.47 \mathrm{E}+00$ & $1.01 \mathrm{E}+01$ \\
\hline C3393-86 & 86.5 & H2 & $5.19 \mathrm{E}+00$ & $8.16 \mathrm{E}-01$ & $2.11 \mathrm{E}+00$ & $5.46 \mathrm{E}+00$ \\
\hline C3393-91 & 91.5 & H2 & $4.46 \mathrm{E}+00$ & $7.34 \mathrm{E}-01$ & $2.39 \mathrm{E}+00$ & $5.09 \mathrm{E}+00$ \\
\hline C3393-96 & 96.5 & H2 & $1.27 \mathrm{E}+01$ & $1.12 \mathrm{E}+00$ & $4.82 \mathrm{E}+00$ & $6.92 \mathrm{E}+00$ \\
\hline C3393-103.5 & 104.0 & H2 & $7.66 \mathrm{E}+00$ & $1.86 \mathrm{E}+00$ & $3.06 \mathrm{E}+00$ & $1.32 \mathrm{E}+01$ \\
\hline C3393-103.5 Dup & 104.0 & H2 & $6.89 \mathrm{E}+00$ & $1.37 \mathrm{E}+00$ & $2.61 \mathrm{E}+00$ & $9.91 \mathrm{E}+00$ \\
\hline C3393-108.5 & 109.0 & H2 & $4.52 \mathrm{E}+00$ & $7.34 \mathrm{E}-01$ & $1.66 \mathrm{E}+00$ & $1.12 \mathrm{E}+01$ \\
\hline C3393-108.5 Dup & 109.0 & H2 & $4.84 \mathrm{E}+00$ & $7.82 \mathrm{E}-01$ & $1.78 \mathrm{E}+00$ & $1.16 \mathrm{E}+01$ \\
\hline C3393-118.5 & 119.0 & H2 & $2.20 \mathrm{E}+00$ & $4.53 \mathrm{E}-01$ & $8.40 \mathrm{E}-01$ & $7.12 \mathrm{E}+00$ \\
\hline C3393-125 & 125.5 & H2 & $3.74 \mathrm{E}+00$ & $9.11 \mathrm{E}-01$ & $1.51 \mathrm{E}+00$ & $9.79 \mathrm{E}+00$ \\
\hline C3393-126 & 126.5 & H2 & $6.54 \mathrm{E}+00$ & $1.58 \mathrm{E}+00$ & $2.49 \mathrm{E}+00$ & $1.60 \mathrm{E}+01$ \\
\hline C3393-136 & 136.5 & CCU & $2.27 \mathrm{E}+00$ & $4.20 \mathrm{E}-01$ & $1.11 \mathrm{E}+00$ & $7.45 \mathrm{E}+00$ \\
\hline C3393-141 & 141.5 & CCU & $2.22 \mathrm{E}+00$ & $3.42 \mathrm{E}-01$ & $1.39 \mathrm{E}+00$ & $5.45 \mathrm{E}+00$ \\
\hline
\end{tabular}

Table 4.9. Calculated Pore Water Mobile Metal Concentrations of Key Contaminants of Concern in the C3393 Borehole Samples

\begin{tabular}{|c|c|c|c|c|c|c|}
\hline $\begin{array}{c}\text { Sample } \\
\text { ID }\end{array}$ & $\begin{array}{l}\text { Mid- } \\
\text { Depth } \\
\mathrm{ft} \text { bgs }\end{array}$ & $\begin{array}{c}\text { Stratigraphic } \\
\text { Unit } \\
\end{array}$ & $\begin{array}{c}\text { Technetium- } \\
99 \\
\text { pCi/L } \\
\end{array}$ & $\begin{array}{c}\text { Uranium- } \\
238 \\
\mu \mathrm{g} / \mathrm{L} \\
\end{array}$ & $\begin{array}{c}\text { Chromium } \\
\mu \mathrm{g} / \mathrm{L}\end{array}$ & $\begin{array}{c}\text { Molybdenum } \\
\mu \mathrm{g} / \mathrm{L}\end{array}$ \\
\hline C3393-6 & 6.5 & H1 & $\overline{(1.25 \mathrm{E}+02)}$ & $7.57 \mathrm{E}+00$ & $\begin{array}{l}(4.93 \mathrm{E}+00) \\
\end{array}$ & $\begin{array}{l}(1.15 \mathrm{E}+01) \\
\end{array}$ \\
\hline C3393-16.5 & 17.0 & H1 & $(8.21 \mathrm{E}+02)$ & $4.34 \mathrm{E}+01$ & $(3.27 \mathrm{E}+00)$ & $(4.27 \mathrm{E}+01)$ \\
\hline C3393-19.25 & 19.0 & $\mathrm{H} 1$ & $(5.40 \mathrm{E}+02)$ & $1.99 \mathrm{E}+01$ & $(6.06 \mathrm{E}+01)$ & $6.27 \mathrm{E}+01$ \\
\hline C3393-51 & 51.5 & $\mathrm{H} 2$ & $(1.59 \mathrm{E}+03)$ & $7.28 \mathrm{E}+00$ & $6.41 \mathrm{E}+02$ & $4.63 \mathrm{E}+02$ \\
\hline C3393-57.5 & 58.0 & $\mathrm{H} 2$ & $(2.89 \mathrm{E}+02)$ & $7.01 \mathrm{E}+00$ & $(9.12 \mathrm{E}+00)$ & $3.84 \mathrm{E}+01$ \\
\hline C3393-71 & 71.5 & $\mathrm{H} 2$ & $(1.13 \mathrm{E}+03)$ & $1.15 \mathrm{E}+01$ & $(3.50 \mathrm{E}+01)$ & $2.02 \mathrm{E}+02$ \\
\hline C3393-76 & 76.5 & $\mathrm{H} 2$ & $(3.57 \mathrm{E}+02)$ & $6.77 \mathrm{E}+00$ & $(1.03 \mathrm{E}+01)$ & $1.26 \mathrm{E}+02$ \\
\hline C3393-83.5 & 84.0 & $\mathrm{H} 2$ & $(6.47 \mathrm{E}+02)$ & $7.04 \mathrm{E}+00$ & $(1.01 \mathrm{E}+01)$ & $2.14 \mathrm{E}+02$ \\
\hline C3393-86 & 86.5 & $\mathrm{H} 2$ & $(3.65 \mathrm{E}+02)$ & $5.83 \mathrm{E}+00$ & $3.96 \mathrm{E}+02$ & $1.71 \mathrm{E}+02$ \\
\hline C3393-91 & 91.5 & $\mathrm{H} 2$ & $(2.14 \mathrm{E}+02)$ & $7.11 \mathrm{E}+00$ & $3.21 \mathrm{E}+01$ & $7.76 \mathrm{E}+01$ \\
\hline C3393-96 & 96.5 & $\mathrm{H} 2$ & $(5.47 \mathrm{E}+02)$ & $5.61 \mathrm{E}+00$ & $(2.60 \mathrm{E}+01)$ & $9.75 \mathrm{E}+01$ \\
\hline C3393-103.5 & 104.0 & $\mathrm{H} 2$ & $(5.11 \mathrm{E}+02)$ & $6.63 \mathrm{E}+00$ & $(6.71 \mathrm{E}+01)$ & $5.12 \mathrm{E}+02$ \\
\hline C3393-103.5 Dup & 104.0 & $\mathrm{H} 2$ & $(4.70 \mathrm{E}+02)$ & $6.19 \mathrm{E}+00$ & $(3.21 \mathrm{E}+01)$ & $3.14 \mathrm{E}+02$ \\
\hline C3393-108.5 & 109.0 & $\mathrm{H} 2$ & $(4.83 \mathrm{E}+02)$ & $8.59 \mathrm{E}+00$ & $(1.16 \mathrm{E}+01)$ & $1.02 \mathrm{E}+02$ \\
\hline C3393-108.5 Dup & 109.0 & $\mathrm{H} 2$ & $(3.05 \mathrm{E}+02)$ & $8.99 \mathrm{E}+00$ & $(3.09 \mathrm{E}+01)$ & $1.16 \mathrm{E}+02$ \\
\hline C3393-118.5 & 119.0 & $\mathrm{H} 2$ & $(1.98 \mathrm{E}+02)$ & $5.19 \mathrm{E}+00$ & $(7.02 \mathrm{E}+00)$ & $8.92 \mathrm{E}+01$ \\
\hline C3393-125 & 125.5 & $\mathrm{H} 2$ & $(3.11 \mathrm{E}+02)$ & $1.17 \mathrm{E}+01$ & $5.28 \mathrm{E}+01$ & $7.47 \mathrm{E}+01$ \\
\hline C3393-126 & 126.5 & $\mathrm{H} 2$ & $(6.90 \mathrm{E}+02)$ & $1.99 \mathrm{E}+01$ & $(5.75 \mathrm{E}+00)$ & $(3.40 \mathrm{E}+01)$ \\
\hline C3393-136 & 136.5 & $\mathrm{CCU}$ & $(2.16 \mathrm{E}+02)$ & $8.93 \mathrm{E}+00$ & $(1.90 \mathrm{E}+01)$ & $2.45 \mathrm{E}+01$ \\
\hline C3393-141 & 141.5 & $\mathrm{CCU}$ & $(1.54 \mathrm{E}+02)$ & $7.33 \mathrm{E}+00$ & $2.75 \mathrm{E}+02$ & $1.88 \mathrm{E}+02$ \\
\hline
\end{tabular}




\subsection{4 $8 \mathrm{M}$ Nitric Acid-Extractable Amounts of Selected Elements in the U Tank Farm Direct Push Sediments}

The same cores and grab samples that were characterized for water-leachable constituents were also characterized to see how much of the various constituents could be extracted with hot $8 \mathrm{M}$ nitric acid (Tables 4.10 through 4.12). The $8 \mathrm{M}$ nitric acid extraction is a protocol used by EPA to estimate the maximum concentrations of regulated metals in contaminated sediment that would be biologically available. Aliquots of sediment from borehole C3393 were subjected to acid extraction to establish baseline values to compare with acid extracts of potentially contaminated sediments from the U Farm direct push holes.

Table 4.10. Acid-Extractable Cations in the U Tank Farm Background Borehole Samples ( $\mu \mathrm{g} / \mathrm{g}$ dry sediment)

\begin{tabular}{||l|c|c|c|c|c|c||}
\hline $\begin{array}{c}\text { Sample } \\
\text { ID }\end{array}$ & $\begin{array}{c}\text { Mid-Depth } \\
\mathrm{ft} \text { bgs }\end{array}$ & Stratigraphic Unit & $\begin{array}{c}\text { Calcium } \\
\mu \mathrm{g} / \mathrm{g}\end{array}$ & $\begin{array}{c}\text { Potassium } \\
\mu \mathrm{g} / \mathrm{g}\end{array}$ & $\begin{array}{c}\text { Magnesium } \\
\mu \mathrm{g} / \mathrm{g}\end{array}$ & $\begin{array}{c}\text { Sodium } \\
\mu \mathrm{g} / \mathrm{g}\end{array}$ \\
\hline \hline C3393-6 & 6.5 & $\mathrm{H} 1$ & $2.04 \mathrm{E}+04$ & $1.54 \mathrm{E}+03$ & $6.20 \mathrm{E}+03$ & $5.35 \mathrm{E}+02$ \\
\hline C3393-16.5 & 17.0 & $\mathrm{H} 1$ & $8.93 \mathrm{E}+03$ & $1.31 \mathrm{E}+03$ & $4.66 \mathrm{E}+03$ & $4.37 \mathrm{E}+02$ \\
\hline C3393-19.25 & 19.0 & $\mathrm{H} 1$ & $1.29 \mathrm{E}+04$ & $1.61 \mathrm{E}+03$ & $4.75 \mathrm{E}+03$ & $5.90 \mathrm{E}+02$ \\
\hline C3393-51 & 51.5 & $\mathrm{H} 2$ & $9.02 \mathrm{E}+03$ & $1.12 \mathrm{E}+03$ & $4.68 \mathrm{E}+03$ & $2.03 \mathrm{E}+02$ \\
\hline C3393-57.5 & 58.0 & $\mathrm{H} 2$ & $1.26 \mathrm{E}+04$ & $2.07 \mathrm{E}+03$ & $6.18 \mathrm{E}+03$ & $2.59 \mathrm{E}+02$ \\
\hline C3393-71 & 71.5 & $\mathrm{H} 2$ & $8.96 \mathrm{E}+03$ & $1.10 \mathrm{E}+03$ & $4.62 \mathrm{E}+03$ & $1.82 \mathrm{E}+02$ \\
\hline C3393-76 & 76.5 & H2 & $9.47 \mathrm{E}+03$ & $2.00 \mathrm{E}+03$ & $5.50 \mathrm{E}+03$ & $2.81 \mathrm{E}+02$ \\
\hline C3393-83.5 & 84.0 & H2 & $9.74 \mathrm{E}+03$ & $1.91 \mathrm{E}+03$ & $5.28 \mathrm{E}+03$ & $2.85 \mathrm{E}+02$ \\
\hline C3393-86 & 86.5 & H2 & $9.97 \mathrm{E}+03$ & $2.41 \mathrm{E}+03$ & $5.72 \mathrm{E}+03$ & $2.55 \mathrm{E}+02$ \\
\hline C3393-91 & 91.5 & H2 & $1.22 \mathrm{E}+04$ & $2.50 \mathrm{E}+03$ & $7.07 \mathrm{E}+03$ & $2.97 \mathrm{E}+02$ \\
\hline C3393-96 & 96.5 & H2 & $9.59 \mathrm{E}+03$ & $2.07 \mathrm{E}+03$ & $5.27 \mathrm{E}+03$ & $2.39 \mathrm{E}+02$ \\
\hline C3393-103.5 & 104.0 & H2 & $9.08 \mathrm{E}+03$ & $1.63 \mathrm{E}+03$ & $4.81 \mathrm{E}+03$ & $2.88 \mathrm{E}+02$ \\
\hline C3393-103.5 Dup & 104.0 & H2 & $9.27 \mathrm{E}+03$ & $1.51 \mathrm{E}+03$ & $4.76 \mathrm{E}+03$ & $2.18 \mathrm{E}+02$ \\
\hline C3393-108.5 & 109.0 & H2 & $7.54 \mathrm{E}+03$ & $2.90 \mathrm{E}+03$ & $6.01 \mathrm{E}+03$ & $2.41 \mathrm{E}+02$ \\
\hline C3393-108.5 Dup & 109.0 & H2 & $7.78 \mathrm{E}+03$ & $2.90 \mathrm{E}+03$ & $6.10 \mathrm{E}+03$ & $2.77 \mathrm{E}+02$ \\
\hline C3393-118.5 & 119.0 & H2 & $1.28 \mathrm{E}+04$ & $2.32 \mathrm{E}+03$ & $6.81 \mathrm{E}+03$ & $2.96 \mathrm{E}+02$ \\
\hline C3393-125 & 125.5 & H2 & $1.27 \mathrm{E}+04$ & $2.18 \mathrm{E}+03$ & $5.89 \mathrm{E}+03$ & $2.98 \mathrm{E}+02$ \\
\hline C3393-126 & 126.5 & H2 & $9.52 \mathrm{E}+03$ & $1.71 \mathrm{E}+03$ & $4.41 \mathrm{E}+03$ & $2.13 \mathrm{E}+02$ \\
\hline C3393-136 & 136.5 & CCU & $1.69 \mathrm{E}+04$ & $2.86 \mathrm{E}+03$ & $8.13 \mathrm{E}+03$ & $3.06 \mathrm{E}+02$ \\
\hline C3393-141 & 141.5 & CCU & $1.26 \mathrm{E}+04$ & $2.72 \mathrm{E}+03$ & $7.99 \mathrm{E}+03$ & $3.18 \mathrm{E}+02$ \\
\hline Sodium values were blank corrected due to contamination resulting from filtration of the samples. \\
\hline
\end{tabular}

Table 4.11. Acid-Leachable Cations in the U Tank Farm Background Borehole Samples $(\mu \mathrm{g} / \mathrm{g}$ dry sediment)

\begin{tabular}{||l|c|c|c|c|c|c||}
\hline $\begin{array}{c}\text { Sample } \\
\text { ID }\end{array}$ & $\begin{array}{c}\text { Mid-Depth } \\
\mathrm{ft} \text { bgs }\end{array}$ & $\begin{array}{c}\text { Stratigraphic } \\
\text { Unit }\end{array}$ & $\begin{array}{c}\text { Aluminum } \\
\mu \mathrm{g} / \mathrm{g}\end{array}$ & $\begin{array}{c}\text { Iron } \\
\mu \mathrm{g} / \mathrm{g}\end{array}$ & $\begin{array}{c}\text { Phosphorus } \\
(\text { as Phosphate) } \\
\mu \mathrm{g} / \mathrm{g}\end{array}$ & $\begin{array}{c}\text { Sulfur } \\
\text { (as Sulfate) } \\
\mu \mathrm{g} / \mathrm{g}\end{array}$ \\
\hline \hline $\mathrm{C} 3393-6$ & 6.5 & $\mathrm{H} 1$ & $1.18 \mathrm{E}+04$ & $2.28 \mathrm{E}+04$ & $2.78 \mathrm{E}+03$ & $9.21 \mathrm{E}+02$ \\
\hline $\mathrm{C} 3393-16.5$ & 17.0 & $\mathrm{H} 1$ & $9.13 \mathrm{E}+03$ & $2.40 \mathrm{E}+04$ & $2.63 \mathrm{E}+03$ & $4.23 \mathrm{E}+02$ \\
\hline $\mathrm{C} 3393-19.25$ & 19.0 & $\mathrm{H} 1$ & $1.16 \mathrm{E}+04$ & $2.38 \mathrm{E}+04$ & $2.86 \mathrm{E}+03$ & $5.23 \mathrm{E}+02$ \\
\hline $\mathrm{C} 3393-51$ & 51.5 & H2 & $6.89 \mathrm{E}+03$ & $1.45 \mathrm{E}+04$ & $1.47 \mathrm{E}+03$ & $4.01 \mathrm{E}+02$ \\
\hline $\mathrm{C} 3393-57.5$ & 58.0 & H2 & $1.08 \mathrm{E}+04$ & $1.77 \mathrm{E}+04$ & $1.87 \mathrm{E}+03$ & $4.95 \mathrm{E}+02$ \\
\hline $\mathrm{C} 3393-71$ & 71.5 & H2 & $6.81 \mathrm{E}+03$ & $1.38 \mathrm{E}+04$ & $1.33 \mathrm{E}+03$ & $3.99 \mathrm{E}+02$ \\
\hline
\end{tabular}




\begin{tabular}{||l|c|c|c|c|c|c||}
\hline $\begin{array}{c}\text { Sample } \\
\text { ID }\end{array}$ & $\begin{array}{c}\text { Mid-Depth } \\
\mathrm{ft} \text { bgs }\end{array}$ & $\begin{array}{c}\text { Stratigraphic } \\
\text { Unit }\end{array}$ & $\begin{array}{c}\text { Aluminum } \\
\mu \mathrm{g} / \mathrm{g}\end{array}$ & $\begin{array}{c}\text { Iron } \\
\mu \mathrm{g} / \mathrm{g}\end{array}$ & $\begin{array}{c}\text { Phosphorus } \\
(\text { as Phosphate) } \\
\mu \mathrm{g} / \mathrm{g}\end{array}$ & $\begin{array}{c}\text { Sulfur } \\
\text { (as Sulfate) } \\
\mu \mathrm{g} / \mathrm{g}\end{array}$ \\
\hline \hline C3393-76 & 76.5 & $\mathrm{H} 2$ & $1.04 \mathrm{E}+04$ & $1.64 \mathrm{E}+04$ & $1.66 \mathrm{E}+03$ & $3.71 \mathrm{E}+02$ \\
\hline C3393-83.5 & 84.0 & $\mathrm{H} 2$ & $1.07 \mathrm{E}+04$ & $1.63 \mathrm{E}+04$ & $1.92 \mathrm{E}+03$ & $3.52 \mathrm{E}+02$ \\
\hline C3393-86 & 86.5 & $\mathrm{H} 2$ & $1.13 \mathrm{E}+04$ & $1.74 \mathrm{E}+04$ & $1.56 \mathrm{E}+03$ & $3.87 \mathrm{E}+02$ \\
\hline C3393-91 & 91.5 & H2 & $1.44 \mathrm{E}+04$ & $2.25 \mathrm{E}+04$ & $2.26 \mathrm{E}+03$ & $4.45 \mathrm{E}+02$ \\
\hline C3393-96 & 96.5 & H2 & $1.01 \mathrm{E}+04$ & $1.64 \mathrm{E}+04$ & $1.60 \mathrm{E}+03$ & $3.56 \mathrm{E}+02$ \\
\hline C3393-103.5 & 104.0 & H2 & $8.80 \mathrm{E}+03$ & $1.50 \mathrm{E}+04$ & $1.63 \mathrm{E}+03$ & $3.63 \mathrm{E}+02$ \\
\hline C3393-103.5 Dup & 104.0 & H2 & $8.76 \mathrm{E}+03$ & $1.52 \mathrm{E}+04$ & $1.75 \mathrm{E}+03$ & $3.85 \mathrm{E}+02$ \\
\hline C3393-108.5 & 109.0 & H2 & $1.12 \mathrm{E}+04$ & $1.80 \mathrm{E}+04$ & $1.72 \mathrm{E}+03$ & $2.92 \mathrm{E}+02$ \\
\hline C3393-108.5 Dup & 109.0 & H2 & $1.18 \mathrm{E}+04$ & $1.84 \mathrm{E}+04$ & $1.76 \mathrm{E}+03$ & $3.08 \mathrm{E}+02$ \\
\hline C3393-118.5 & 119.0 & H2 & $1.34 \mathrm{E}+04$ & $2.12 \mathrm{E}+04$ & $2.44 \mathrm{E}+03$ & $4.41 \mathrm{E}+02$ \\
\hline C3393-125 & 125.5 & H2 & $1.18 \mathrm{E}+04$ & $1.86 \mathrm{E}+04$ & $1.84 \mathrm{E}+03$ & $4.29 \mathrm{E}+02$ \\
\hline C3393-126 & 126.5 & H2 & $8.00 \mathrm{E}+03$ & $1.36 \mathrm{E}+04$ & $1.41 \mathrm{E}+03$ & $3.43 \mathrm{E}+02$ \\
\hline C3393-136 & 136.5 & CCU & $1.90 \mathrm{E}+04$ & $2.44 \mathrm{E}+04$ & $1.65 \mathrm{E}+03$ & $5.78 \mathrm{E}+02$ \\
\hline C3393-141 & 141.5 & CCU & $1.75 \mathrm{E}+04$ & $2.06 \mathrm{E}+04$ & $1.73 \mathrm{E}+03$ & $4.14 \mathrm{E}+02$ \\
\hline
\end{tabular}

Table 4.12. Acid-Extractable Mobile Metals in the U Tank Farm Background Borehole Samples ( $\mu \mathrm{g} / \mathrm{g}$ dry sediment)

\begin{tabular}{|c|c|c|c|c|c|c|c|}
\hline $\begin{array}{l}\text { Sample } \\
\text { ID }\end{array}$ & $\begin{array}{l}\text { Mid- } \\
\text { Depth } \\
\mathrm{ft} \text { bgs }\end{array}$ & Stratigraphic Unit & $\begin{array}{c}\text { Technetium- } \\
99 \\
\text { pCi/g }\end{array}$ & $\begin{array}{c}\text { Uranium- } \\
238 \\
\mu \mathrm{g} / \mathrm{g}\end{array}$ & $\begin{array}{c}\text { Molybdenum } \\
\mu \mathrm{g} / \mathrm{g}\end{array}$ & $\begin{array}{l}\text { Chromium } \\
\mu \mathrm{g} / \mathrm{g}\end{array}$ & $\begin{array}{l}\text { Lead } \\
\mu \mathrm{g} / \mathrm{g}\end{array}$ \\
\hline C3393-6 & 6.5 & H1 & $(7.09 \mathrm{E}+00)$ & 8.46E-01 & $9.01 \mathrm{E}-02$ & $1.98 \mathrm{E}+00$ & $7.51 \mathrm{E}+00$ \\
\hline C3393-16.5 & 17.0 & $\mathrm{H} 1$ & $(4.23 \mathrm{E}+00)$ & 4.74E-01 & $9.00 \mathrm{E}-02$ & $1.27 \mathrm{E}+00$ & $3.11 \mathrm{E}+00$ \\
\hline C3393-19.25 & 19.0 & H1 & $(4.48 \mathrm{E}+00)$ & $5.73 \mathrm{E}-01$ & $6.94 \mathrm{E}-02$ & $1.28 \mathrm{E}+00$ & $4.20 \mathrm{E}+00$ \\
\hline C3393-51 & 51.5 & $\mathrm{H} 2$ & $(4.06 \mathrm{E}+00)$ & $4.75 \mathrm{E}-01$ & $5.41 \mathrm{E}-02$ & $1.84 \mathrm{E}+00$ & $3.65 \mathrm{E}+00$ \\
\hline C3393-57.5 & 58.0 & $\mathrm{H} 2$ & $(5.94 \mathrm{E}+00)$ & $7.46 \mathrm{E}-01$ & $7.00 \mathrm{E}-02$ & $2.16 \mathrm{E}+00$ & $7.78 \mathrm{E}+00$ \\
\hline C3393-71 & 71.5 & $\mathrm{H} 2$ & $(4.06 \mathrm{E}+00)$ & $4.74 \mathrm{E}-01$ & $6.31 \mathrm{E}-02$ & $1.84 \mathrm{E}+00$ & $3.95 \mathrm{E}+00$ \\
\hline C3393-76 & 76.5 & $\mathrm{H} 2$ & $(3.64 \mathrm{E}+00)$ & $6.37 \mathrm{E}-01$ & $6.46 \mathrm{E}-02$ & $2.40 \mathrm{E}+00$ & $5.04 \mathrm{E}+00$ \\
\hline C3393-83.5 & 84.0 & $\mathrm{H} 2$ & $(5.74 \mathrm{E}+00)$ & $7.28 \mathrm{E}-01$ & $8.08 \mathrm{E}-02$ & $2.42 \mathrm{E}+00$ & $5.57 \mathrm{E}+00$ \\
\hline C3393-86 & 86.5 & $\mathrm{H} 2$ & $(3.63 \mathrm{E}+00)$ & $5.97 \mathrm{E}-01$ & $7.67 \mathrm{E}-02$ & $2.26 \mathrm{E}+00$ & $5.57 \mathrm{E}+00$ \\
\hline C3393-91 & 91.5 & $\mathrm{H} 2$ & $(5.46 \mathrm{E}+00)$ & $8.65 \mathrm{E}-01$ & $8.80 \mathrm{E}-02$ & $2.37 \mathrm{E}+00$ & $9.26 \mathrm{E}+00$ \\
\hline C3393-96 & 96.5 & $\mathrm{H} 2$ & $(4.68 \mathrm{E}+00)$ & $6.11 \mathrm{E}-01$ & $1.06 \mathrm{E}-01$ & $2.11 \mathrm{E}+00$ & $4.81 \mathrm{E}+00$ \\
\hline C3393-103.5 & 104.0 & $\mathrm{H} 2$ & $(5.19 \mathrm{E}+00)$ & $6.43 \mathrm{E}-01$ & $1.44 \mathrm{E}-01$ & $2.33 \mathrm{E}+00$ & $5.14 \mathrm{E}+00$ \\
\hline C3393-103.5 Dup & 104.0 & $\mathrm{H} 2$ & $(5.17 \mathrm{E}+00)$ & $7.30 \mathrm{E}-01$ & $8.79 \mathrm{E}-02$ & $2.32 \mathrm{E}+00$ & $5.29 \mathrm{E}+00$ \\
\hline C3393-108.5 & 109.0 & $\mathrm{H} 2$ & $(4.72 \mathrm{E}+00)$ & $5.06 \mathrm{E}-01$ & $6.22 \mathrm{E}-02$ & $2.44 \mathrm{E}+00$ & $5.01 \mathrm{E}+00$ \\
\hline C3393-108.5 Dup & 109.0 & $\mathrm{H} 2$ & $(4.99 \mathrm{E}+00)$ & $5.45 \mathrm{E}-01$ & $6.50 \mathrm{E}-02$ & $2.52 \mathrm{E}+00$ & $5.12 \mathrm{E}+00$ \\
\hline C3393-118.5 & 119.0 & $\mathrm{H} 2$ & $(4.88 \mathrm{E}+00)$ & $7.56 \mathrm{E}-01$ & $8.82 \mathrm{E}-02$ & $2.87 \mathrm{E}+00$ & $8.07 \mathrm{E}+00$ \\
\hline C3393-125 & 125.5 & $\mathrm{H} 2$ & $(5.16 \mathrm{E}+00)$ & $7.82 \mathrm{E}-01$ & $5.44 \mathrm{E}-02$ & $1.86 \mathrm{E}+00$ & $6.50 \mathrm{E}+00$ \\
\hline C3393-126 & 126.5 & $\mathrm{H} 2$ & $(5.16 \mathrm{E}+00)$ & 4.94E-01 & $4.10 \mathrm{E}-02$ & $1.62 \mathrm{E}+00$ & $4.44 \mathrm{E}+00$ \\
\hline C3393-136 & 136.5 & $\mathrm{CCU}$ & $(5.16 \mathrm{E}+00)$ & $8.41 \mathrm{E}-01$ & $5.91 \mathrm{E}-02$ & $1.91 \mathrm{E}+00$ & $1.52 \mathrm{E}+01$ \\
\hline C3393-141 & 141.5 & $\mathrm{CCU}$ & $(4.12 \mathrm{E}+00)$ & $9.73 \mathrm{E}-01$ & $4.71 \mathrm{E}-02$ & $2.01 \mathrm{E}+00$ & $1.31 \mathrm{E}+01$ \\
\hline
\end{tabular}

None of the major cations or key contaminants of concern appeared to be elevated in acid extracts of the background borehole samples. As seen in Table 4.10, total acid-extractable calcium concentrations exceed those for sodium by one to two orders of magnitude. Although sodium was the dominant waterextractable cation in most of these samples, the large calcium content in the samples was clearly evident 
when a more aggressive extraction procedure was used. These results further support the conclusion that the water-extractable sodium was likely an artifact of contamination by a moderate concentration sodiumbearing waste stream. A comparison of the water-leachable and acid-leachable contents of the sediments from borehole $\mathrm{C} 3393$ showed that less than $0.1 \%$ of the acid-extractable quantities of the following elements were water leachable: aluminum, barium, chromium, iron, manganese, and phosphorous as phosphate. Less than $1 \%$ of the acid-extractable quantities of the following elements were water leachable: calcium, potassium, magnesium, nickel, strontium, zinc, and uranium. Less than $10 \%$ of the acid-extractable sulfur (as sulfate) was water soluble and less than $15 \%$ of the acid-extractable sodium was water-extractable.

\subsubsection{Radionuclide Content in Vadose Zone Sediment from Borehole C3393}

The sediment cores from borehole C3393 did not contain any anthropogenic elements that produce gamma radioactivity. The uranium measured in these samples has been attributed to natural uranium. The radioanalytical analyses performed on the sediment included direct gamma energy analysis and technetium-99 and uranium-238 analysis of the 1:1 sediment:water extracts and the sediment:acid extracts. The uranium and technetium results are shown in Table 4.6 (water-extractable) and 4.12 (acidextractable). Both data sets indicated no elevated amounts are present (less than $1 \mu \mathrm{g} / \mathrm{g}$ acid-extractable uranium is typical for uncontaminated Hanford sediments). The GEA data were not reported because there was nothing significant to report.

\subsubsection{Total Carbon, Calcium Carbonate, and Organic Carbon Content of Vadose Zone Sediment from Borehole C3393}

Table 4.13 shows the total carbon, inorganic carbon, and organic carbon contents of the vadose zone sediment collected from borehole $\mathrm{C} 3393$. The inorganic carbon was also converted to the equivalent calcium-carbonate content. The sediment in the Hanford formation $\mathrm{H} 1$ and $\mathrm{H} 2$ units was relatively low in calcium carbonate $(<3 \mathrm{wt} \%)$, with little to no organic carbon. It was surprising that one sample collected from the Hanford formation $\mathrm{H} 1$ unit contained 3.99\% inorganic carbon $\left(\right.$ as $\mathrm{CaCO}_{3}$ ) versus $3.94 \%$ and $3.54 \%$ for the two samples collected from the carbonate-rich Cold Creek Unit. It is possible that the inorganic carbon content measured in the sample collected from approximately $6 \mathrm{ft}$ bgs could be elevated due to contamination by a waste source.

Table 4.13. Total, Inorganic, and Organic Carbon Content of Vadose Zone Sediments from Borehole C3393

\begin{tabular}{|l|c|c|c|c|c|c||}
\hline $\begin{array}{c}\text { Sample } \\
\text { ID }\end{array}$ & $\begin{array}{c}\text { Mid-Depth } \\
\text { ft bgs }\end{array}$ & $\begin{array}{c}\text { Stratigraphic } \\
\text { Unit }\end{array}$ & $\begin{array}{c}\text { Total } \\
\text { Carbon } \\
(\%)\end{array}$ & $\begin{array}{c}\text { Inorganic } \\
\text { Carbon } \\
(\%)\end{array}$ & $\begin{array}{c}\text { Inorganic Carbon } \\
\text { as } \text { CaCO }_{3} \\
(\%)\end{array}$ & $\begin{array}{c}\text { Organic } \\
\text { Carbon } \\
\text { (by difference) }\end{array}$ \\
\hline \hline C3393-6 & 6.5 & H1 & $5.90 \mathrm{E}-01$ & $4.79 \mathrm{E}-01$ & $3.99 \mathrm{E}+00$ & $1.10 \mathrm{E}-01$ \\
\hline C3393-16.5 & 17.0 & H1 & $1.90 \mathrm{E}-01$ & $1.59 \mathrm{E}-01$ & $1.33 \mathrm{E}+00$ & $3.00 \mathrm{E}-02$ \\
\hline C3393-19.25 & 19.0 & H1 & $2.40 \mathrm{E}-01$ & $1.89 \mathrm{E}-01$ & $1.58 \mathrm{E}+00$ & $5.00 \mathrm{E}-02$ \\
\hline C3393-51 & 51.5 & H2 & $2.60 \mathrm{E}-01$ & $2.05 \mathrm{E}-01$ & $1.71 \mathrm{E}+00$ & $5.00 \mathrm{E}-02$ \\
\hline C3393-57.5 & 58.0 & H2 & $3.70 \mathrm{E}-01$ & $2.74 \mathrm{E}-01$ & $2.29 \mathrm{E}+00$ & $1.00 \mathrm{E}-01$ \\
\hline C3393-71 & 71.5 & H2 & $2.72 \mathrm{E}-01$ & $2.02 \mathrm{E}-01$ & $1.68 \mathrm{E}+00$ & $7.00 \mathrm{E}-02$ \\
\hline C3393-76 & 76.5 & H2 & $2.30 \mathrm{E}-01$ & $1.77 \mathrm{E}-01$ & $1.48 \mathrm{E}+00$ & $5.00 \mathrm{E}-02$ \\
\hline C3393-83.5 & 84.0 & H2 & $2.60 \mathrm{E}-01$ & $2.09 \mathrm{E}-01$ & $1.74 \mathrm{E}+00$ & $5.00 \mathrm{E}-02$ \\
\hline C3393-86 & 86.5 & H2 & $2.80 \mathrm{E}-01$ & $2.18 \mathrm{E}-01$ & $1.82 \mathrm{E}+00$ & $6.00 \mathrm{E}-02$ \\
\hline
\end{tabular}




\begin{tabular}{|l|c|c|c|c|c|c||}
\hline $\begin{array}{c}\text { Sample } \\
\text { ID }\end{array}$ & $\begin{array}{c}\text { Mid-Depth } \\
\text { ft bgs }\end{array}$ & $\begin{array}{c}\text { Stratigraphic } \\
\text { Unit }\end{array}$ & $\begin{array}{c}\text { Total } \\
\text { Carbon } \\
(\%)\end{array}$ & $\begin{array}{c}\text { Inorganic } \\
\text { Carbon } \\
(\%)\end{array}$ & $\begin{array}{c}\text { Inorganic Carbon } \\
\text { as } \mathrm{CaCO}_{3} \\
(\%)\end{array}$ & $\begin{array}{c}\text { Organic } \\
\text { Carbon } \\
\text { (by difference) }\end{array}$ \\
\hline C3393-91 & 91.5 & $\mathrm{H} 2$ & $2.90 \mathrm{E}-01$ & $2.39 \mathrm{E}-01$ & $1.99 \mathrm{E}+00$ & $5.00 \mathrm{E}-02$ \\
\hline C3393-96 & 96.5 & $\mathrm{H} 2$ & $2.40 \mathrm{E}-01$ & $1.79 \mathrm{E}-01$ & $1.49 \mathrm{E}+00$ & $6.00 \mathrm{E}-02$ \\
\hline C3393-103.5 & 104.0 & $\mathrm{H} 2$ & $2.60 \mathrm{E}-01$ & $2.13 \mathrm{E}-01$ & $1.78 \mathrm{E}+00$ & $5.00 \mathrm{E}-02$ \\
\hline C3393-103.5 Dup & 104.0 & H2 & $2.50 \mathrm{E}-01$ & $1.89 \mathrm{E}-01$ & $1.58 \mathrm{E}+00$ & $6.00 \mathrm{E}-02$ \\
\hline C3393-108.5 & 109.0 & H2 & $2.30 \mathrm{E}-01$ & $1.71 \mathrm{E}-01$ & $1.43 \mathrm{E}+00$ & $6.00 \mathrm{E}-02$ \\
\hline C3393-108.5 Dup & 109.0 & H2 & $2.20 \mathrm{E}-01$ & $1.67 \mathrm{E}-01$ & $1.39 \mathrm{E}+00$ & $5.00 \mathrm{E}-02$ \\
\hline C3393-118.5 & 119.0 & H2 & $2.90 \mathrm{E}-01$ & $2.41 \mathrm{E}-01$ & $2.01 \mathrm{E}+00$ & $5.00 \mathrm{E}-02$ \\
\hline C3393-125 & 125.5 & H2 & $2.90 \mathrm{E}-01$ & $2.28 \mathrm{E}-01$ & $1.90 \mathrm{E}+00$ & $6.00 \mathrm{E}-02$ \\
\hline C3393-126 & 126.5 & H2 & $2.80 \mathrm{E}-01$ & $2.10 \mathrm{E}-01$ & $1.75 \mathrm{E}+00$ & $7.00 \mathrm{E}-02$ \\
\hline C3393-136 & 136.5 & CCU & $5.62 \mathrm{E}-01$ & $4.73 \mathrm{E}-01$ & $3.94 \mathrm{E}+00$ & $9.00 \mathrm{E}-02$ \\
\hline C3393-141 & 141.5 & CCU & $4.67 \mathrm{E}-01$ & $4.25 \mathrm{E}-01$ & $3.54 \mathrm{E}+00$ & $5.00 \mathrm{E}-02$ \\
\hline
\end{tabular}

\subsubsection{Particle Size Measurements on Vadose Zone Sediment}

Hydrometer and wet sieving methods were used to determine the particle-size distributions of samples from borehole C3393. Wet sieving results are shown in Table 4.14 and the particle-size distribution data from both techniques are shown in Table 4.15 and Figure 4.1 as a plot of "cumulative percent finer than" versus "particle size in micrometers." The one CCU sample analyzed, with a median grain size of $\sim 35$ micrometers, was the finest grained sediment of the five samples characterized (one Hanford formation $\mathrm{H} 1$ unit sample, three Hanford formation $\mathrm{H} 2$ unit samples, and one CCU sample). The coarsest sample analyzed was the one sample from Hanford formation H1 unit. This sample, collected from $58 \mathrm{ft}$ bgs, had a median grain size of approximately 250 micrometers. Comparatively, the three samples analyzed from the Hanford formation $\mathrm{H} 2$ unit had median grain sizes of approximately 100 to 150 micrometers. The three samples analyzed within the Hanford formation $\mathrm{H} 2$ unit had a large range of sediment moisture contents (4.93 wt\% to $11.6 \mathrm{wt} \%$ ), and surprisingly, the sediment moisture content could not be directly correlated with particle size or specifically, the percentage of silt or clay present in each sample. While there are general trends in which sediments with finer-grained compositions have higher moisture contents (sample C3393-141), discrepancies within this limited data set do not permit an estimate of median particle size based on sediment moisture content.

Table 4.14. Wet Sieve Particle Size Results for Borehole C3393 Sediments

\begin{tabular}{|c|c|c|c|c|c|c|}
\hline \multirow[b]{2}{*}{ Sample ID } & \multirow{2}{*}{$\begin{array}{l}\text { Mid-Depth } \\
\quad(\mathrm{ft} \text { bgs })\end{array}$} & \multirow{2}{*}{$\begin{array}{c}\text { Moisture Content } \\
(\%)\end{array}$} & \multirow{2}{*}{$\begin{array}{c}\text { Stratigraphic } \\
\text { Unit }\end{array}$} & \multicolumn{3}{|c|}{ Weight Percent } \\
\hline & & & & Gravel & Sand & Silt/Clay \\
\hline C3393-57.5 & $\overline{58.0}$ & 14.7 & H1 & 0.16 & 73.2 & 25.6 \\
\hline C3393-86 & 86.5 & 11.6 & $\mathrm{H} 2$ & 0 & 72.5 & 27.3 \\
\hline C3393-108.5 & 109.0 & 8.80 & $\mathrm{H} 2$ & 0 & 85.5 & 14.5 \\
\hline C3393-126 & 126.5 & 4.93 & $\mathrm{H} 2$ & 0 & 81.0 & 18.8 \\
\hline C3393-141 & 141.5 & 22.1 & $\mathrm{CCU}$ & 0 & 31.0 & 67.0 \\
\hline
\end{tabular}


Table 4.15. Particle Size Data for Borehole C3393 Sediments Using Two Techniques Reported as Cumulative Percent Finer Than

\begin{tabular}{|c|c|c|c|c|c|c|c|c|c|}
\hline \multicolumn{2}{|c|}{ Unit: $\mathrm{H} 1$} & \multicolumn{2}{|c|}{$\mathrm{H} 2$} & \multicolumn{2}{|c|}{$\mathrm{H} 2$} & \multicolumn{2}{|c|}{$\mathrm{H} 2$} & \multicolumn{2}{|c|}{$\mathrm{CCU}$} \\
\hline \multicolumn{2}{|c|}{ Depth (ft bgs): 58.0} & \multicolumn{2}{|c|}{86.5} & \multicolumn{2}{|c|}{109.0} & \multicolumn{2}{|c|}{126.5} & \multicolumn{2}{|c|}{141.5} \\
\hline $\begin{array}{c}\text { Diameter } \\
(\mu \mathrm{m})\end{array}$ & $\begin{array}{c}\% \\
\text { finer than }\end{array}$ & $\begin{array}{c}\text { Diameter } \\
(\mu \mathrm{m})\end{array}$ & $\begin{array}{c}\% \\
\text { finer than }\end{array}$ & $\begin{array}{c}\text { Diameter } \\
(\mu \mathrm{m})\end{array}$ & $\begin{array}{c}\% \\
\text { finer than }\end{array}$ & $\begin{array}{c}\text { Diameter } \\
(\mu \mathrm{m})\end{array}$ & $\begin{array}{c}\% \\
\text { finer than }\end{array}$ & $\begin{array}{c}\text { Diameter } \\
(\mu \mathrm{m})\end{array}$ & $\begin{array}{c}\% \\
\text { finer than }\end{array}$ \\
\hline \multicolumn{10}{|c|}{ Wet Sieve } \\
\hline 2000 & 98.8 & 2000 & 100.0 & 2000 & 100.0 & 2000 & 100.0 & 2000 & 100.0 \\
\hline 1000 & 91.0 & 1000 & 99.9 & 1000 & 99.9 & 1000 & 100.0 & \begin{tabular}{|l|}
1000 \\
\end{tabular} & 99.7 \\
\hline 500 & 82.4 & 500 & 99.8 & 500 & 99.6 & 500 & 99.3 & 500 & 98.6 \\
\hline 250 & 52.9 & 250 & 99.5 & 250 & 95.0 & 250 & 85.6 & 250 & 96.0 \\
\hline 106 & 39.3 & 106 & 48.6 & 106 & 27.3 & 106 & 40.4 & 106 & 91.7 \\
\hline 75 & 26.6 & 75 & 37.6 & 75 & 22.4 & 75 & 28.7 & 75 & 87.0 \\
\hline 53 & 98.8 & 53 & 27.5 & 53 & 14.5 & 53 & 19.0 & 53 & 69.0 \\
\hline \multicolumn{10}{|c|}{ Hydrometer } \\
\hline 91.17 & 32.7 & 89.09 & 38.1 & 90.41 & 37.6 & 84.47 & 26.2 & 91.6 & 88.4 \\
\hline 62.06 & 22.2 & 61.28 & 27.8 & 59.66 & 18.0 & 57.51 & 13.1 & 63.5 & 76.1 \\
\hline 34.53 & 12.7 & 34.66 & 20.5 & 33.43 & 9.80 & 32.77 & 8.72 & 35.1 & 48.1 \\
\hline 18.63 & 8.98 & 18.74 & 16.1 & 18.15 & 7.62 & 17.87 & 7.27 & 18.6 & 31.3 \\
\hline 10.64 & 6.34 & 10.73 & 11.7 & 10.34 & 4.35 & 10.27 & 5.81 & 10.5 & 20.1 \\
\hline 7.49 & 5.28 & 7.52 & 8.79 & 7.31 & 4.35 & 7.26 & 5.81 & 7.38 & 15.7 \\
\hline 6.09 & 4.23 & 6.11 & 7.32 & 5.97 & 4.35 & 5.91 & 5.81 & 6.02 & 15.7 \\
\hline 5.26 & 3.70 & 5.28 & 6.59 & 5.16 & 3.27 & 5.10 & 3.63 & 5.19 & 13.4 \\
\hline 1.51 & 3.17 & 1.51 & 2.93 & 1.47 & 1.63 & 1.46 & 1.45 & 1.48 & 6.71 \\
\hline
\end{tabular}

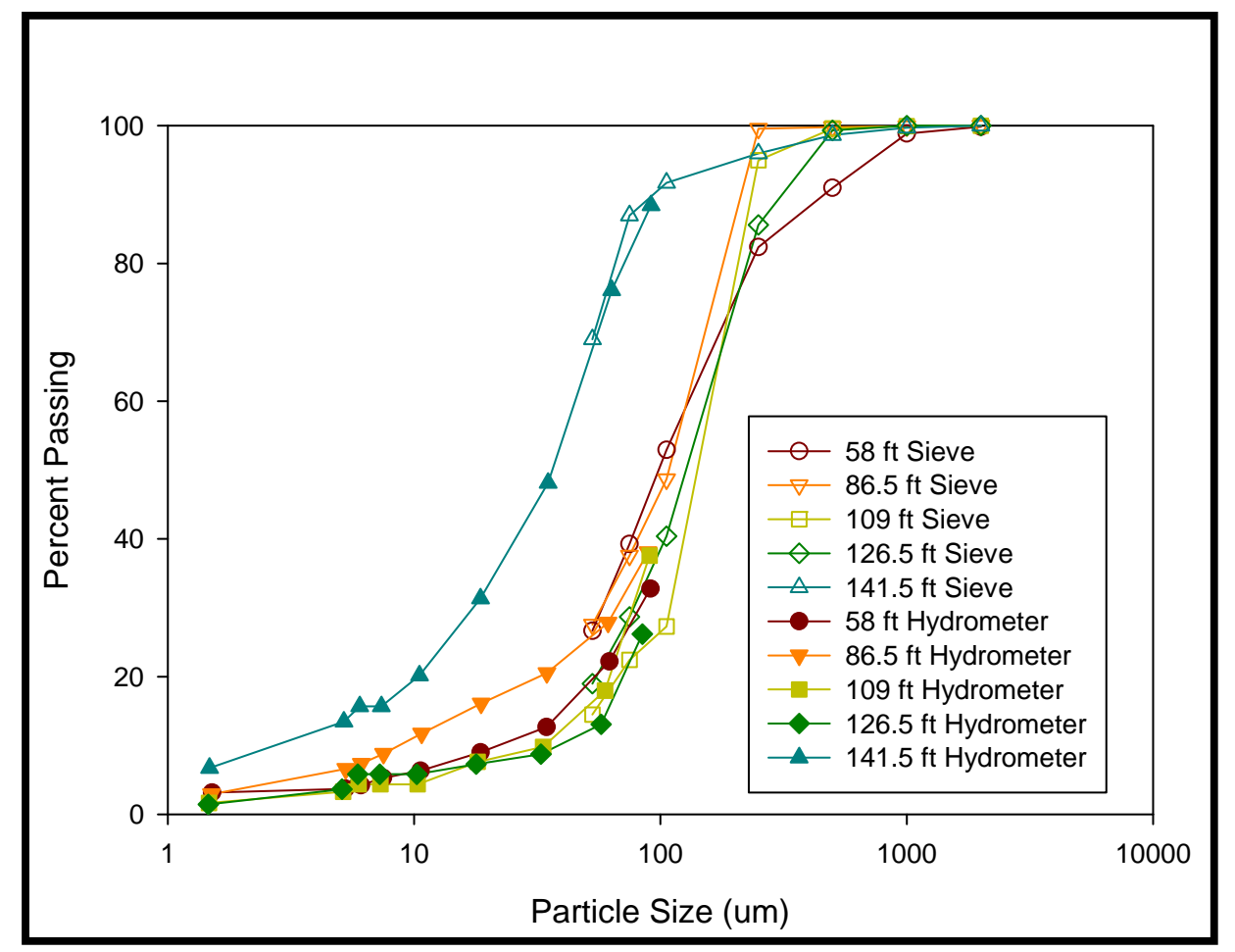

Figure 4.1. Particle-Size Distribution of Sediment Sub-Samples from Borehole C3393 


\subsubsection{Matric Suction Potential Measurements}

Water potential measurements have been included in the Hanford Tank Farm Vadose Zone Characterization Program to document the potential energy state of pore waters in the tank farm sediments. At the tank farms, vegetation is absent, surface soils are coarse-textured, and the likelihood for drainage (recharge) is high (Gee 1987; Gee et al. 1992). However, actual drainage rates are generally unknown. The status of soil water can be defined by either the amount of water in the soil (water content) or by the force that holds water to the soil matrix (i.e., the matric potential or suction) (Or and Wraith 2002). In recent studies, Serne et al. (2002b, 2002c, 2002e, and 2002f) and Lindenmeier et al. (2002) measured both water content (gravimetrically) and matric water potential (filter paper method, ASTM 2002) on core samples obtained from boreholes in the SX and B-BX Tank Farm environs. The same measurements were made at borehole C3393 near the U Tank Farm. At C3393, continuous coring was performed from approximately 3 to $145 \mathrm{ft}$ bgs. The water table in this region was at $227 \mathrm{ft}$ bgs.

Table 4.16 and Figure 4.2 show the matric potentials as a function of depth. Also plotted in Figure 4.2 is the gravity head expressed in pressure units (MPa). The gravity head is zero at the water table and increases linearly with height to the soil surface. For the core samples available from borehole C3393, the water potentials are generally much less than the gravity potential from the shallowest core at $3 \mathrm{ft}$ bgs down to the deepest core taken at $144 \mathrm{ft}$ bgs, representing both the Hanford formation $\mathrm{H} 1$ and $\mathrm{H} 2$ units and the CCU. The red line, labeled "theoretical value" in Figure 4.2, is the theoretical line that represents the steady-state unit gradient condition. This condition represents the profile for matric potential in a sediment profile that is neither draining nor drier than (actively evapotranspiring) a profile at equilibrium. Matric potential values to the left of the unit gradient line suggest a draining profile. One sample (C3393-43.5) had a calculated matric potential above the theoretical line; otherwise, the general trend for the data from $\mathrm{C} 3393$ is that the water potentials are consistent with a draining profile.

Table 4.16. Matric Potential as Measured by Filter Paper Method for Borehole C3393 Core Sediments

\begin{tabular}{|l|c|c|c|c||}
\hline Sample ID & $\begin{array}{c}\text { Mid Depth } \\
\text { (ft bgs) }\end{array}$ & $\begin{array}{c}\text { Stratigraphic Unit } \\
\text { (Hanford formation) }\end{array}$ & $\begin{array}{c}\text { Matric Potential } \\
\text { (MPa) }\end{array}$ & $\begin{array}{c}\text { Theoretical } \\
\text { Potential (MPa) }\end{array}$ \\
\hline C3393-4 & 4.5 & H1 & 0.0130 & 0.6782 \\
\hline C3393-8.5 & 9.0 & H1 & 0.1070 & 0.6645 \\
\hline C3393-14 & 14.5 & H1 & 0.0528 & 0.6477 \\
\hline C3393-18.5 & 19.0 & H1 & 0.2280 & 0.6340 \\
\hline C3393-23.5 & 24.0 & H1 & 0.0578 & 0.6187 \\
\hline C3393-29 & 29.5 & H1 & 0.0467 & 0.6020 \\
\hline C3393-34 & 34.5 & H1 & 0.0433 & 0.5867 \\
\hline C3393-38.5 & 39.0 & H1 & 0.0977 & 0.5730 \\
\hline C3393-43.5 & 44.0 & H1 & 0.5999 & 0.5578 \\
\hline C3393-48.5 & 49.0 & H1 & 0.4847 & 0.5425 \\
\hline C3393-53.5 & 54.0 & H2 & 0.2178 & 0.5273 \\
\hline C3393-58.5 & 59.0 & H2 & 0.0065 & 0.5121 \\
\hline C3393-63.5 & 64.0 & $\mathrm{H} 2$ & 0.2713 & 0.4968 \\
\hline C3393-68.5 & 69.0 & $\mathrm{H} 2$ & 0.3115 & 0.4816 \\
\hline C3393-73.5 & 74.0 & $\mathrm{H} 2$ & 0.1045 & 0.4663 \\
\hline C3393-78.5 & 79.0 & $\mathrm{H} 2$ & 0.0246 & 0.4511 \\
\hline C3393-83.5 & 84.0 & $\mathrm{H} 2$ & 0.0479 & 0.4359 \\
\hline
\end{tabular}




\begin{tabular}{|l|c|c|c|c||}
\hline $\mathrm{C} 3393-88.5$ & 89.0 & $\mathrm{H} 2$ & 0.0769 & 0.4206 \\
\hline $\mathrm{C} 3393-93.5$ & 94.0 & $\mathrm{H} 2$ & 0.0879 & 0.4054 \\
\hline $\mathrm{C} 3393-98.5$ & 99.0 & $\mathrm{H} 2$ & 0.0172 & 0.3901 \\
\hline $\mathrm{C} 3393-103.5$ & 104.0 & $\mathrm{H} 2$ & 0.0311 & 0.3749 \\
\hline $\mathrm{C} 3393-108.5$ & 109.0 & $\mathrm{H} 2$ & 0.0095 & 0.3597 \\
\hline $\mathrm{C} 3393-113.5$ & 114.0 & $\mathrm{H} 2$ & 0.0288 & 0.3444 \\
\hline $\mathrm{C} 3393-118.5$ & 119.0 & $\mathrm{H} 2$ & 0.0071 & 0.3292 \\
\hline $\mathrm{C} 3393-123.5$ & 124.0 & $\mathrm{H} 2$ & 0.2007 & 0.3139 \\
\hline $\mathrm{C} 3393-128.5$ & 129.0 & $\mathrm{H} 2$ & 0.1751 & 0.2987 \\
\hline $\mathrm{C} 3393-133.5$ & 134.0 & $\mathrm{CCU}$ & 0.0839 & 0.2835 \\
\hline $\mathrm{C} 3393-138.5$ & 139.0 & $\mathrm{CCU}$ & 0.0329 & 0.2682 \\
\hline $\mathrm{C} 3393-143.5$ & 144.0 & $\mathrm{CCU}$ & 0.0144 & 0.2530 \\
\hline
\end{tabular}

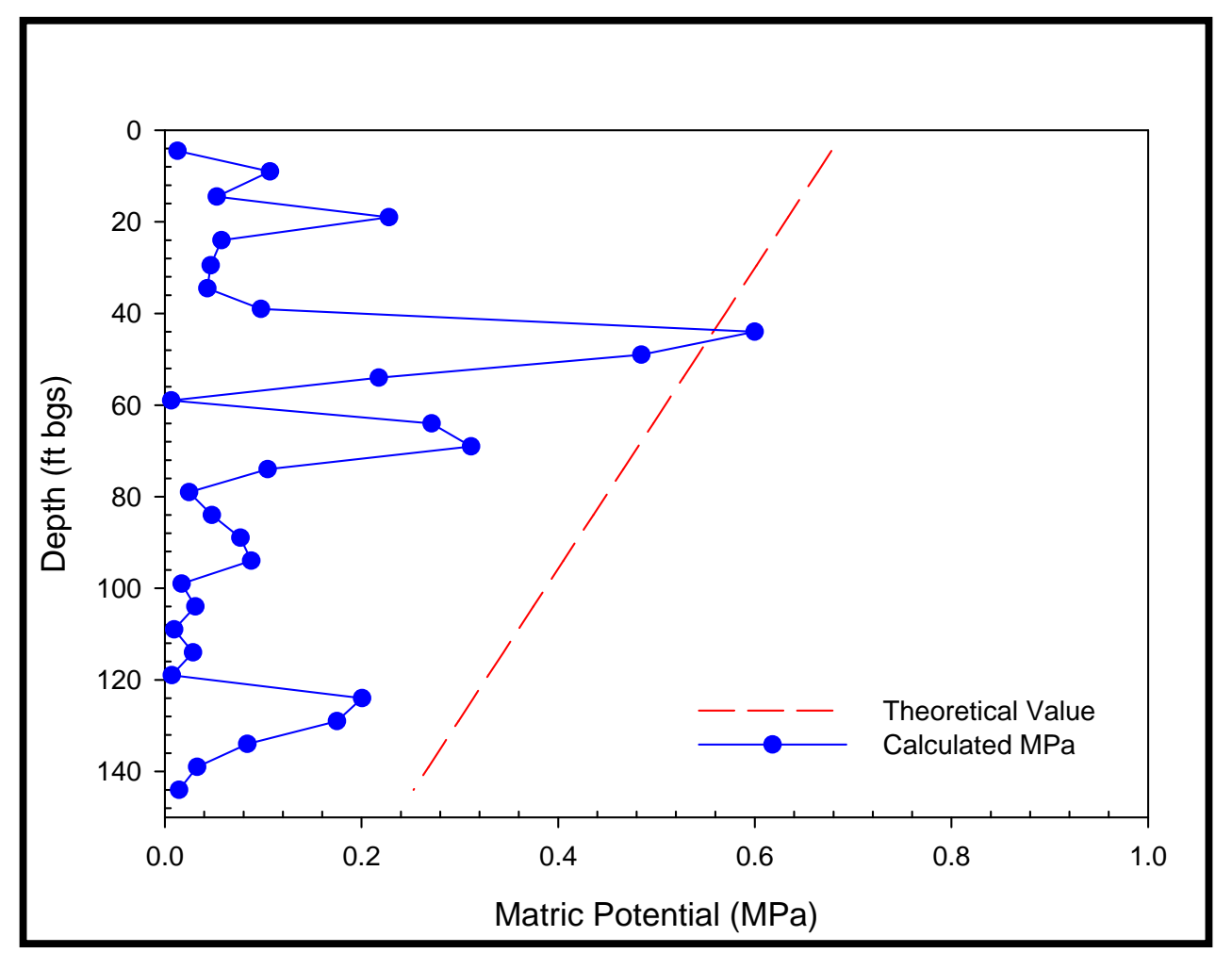

Figure 4.2. Matric Water Potential Measured by Filter Paper Technique on Core Samples from Borehole C3393

\subsubsection{Ammonium Acetate Extractions}

The exchangeable fraction of cations in samples from the background borehole is presented in Table 4.17 in units of $\mu \mathrm{g} / \mathrm{g}$ and Table 4.18 in units of meq/100g of dry sediment. Reproducibility of the measurements, as observed through duplicate analysis of samples, was excellent. Calcium was the dominant ammonium-acetate-extractable cation in all of the samples analyzed. The total calculated average cation exchange capacities for the duplicate samples ranged from a low of 6.34 to a high of $9.84 \mathrm{meq} / 100 \mathrm{~g}$. 
Table 4.17. Ammonium Acetate-Extractable Cations in the U Tank Farm Background Borehole Samples $(\mu \mathrm{g} / \mathrm{g}$ dry sediment)

\begin{tabular}{||l|c|c|c|c|c|c|c||}
\hline $\begin{array}{c}\text { Sample } \\
\text { ID }\end{array}$ & $\begin{array}{c}\text { Mid-Depth } \\
\mathrm{ft} \text { bgs }\end{array}$ & $\begin{array}{c}\text { Stratigraphic } \\
\text { Unit }\end{array}$ & $\begin{array}{c}\text { Barium } \\
\mu \mathrm{g} / \mathrm{g}\end{array}$ & $\begin{array}{c}\text { Calcium } \\
\mu \mathrm{g} / \mathrm{g}\end{array}$ & $\begin{array}{c}\text { Potassium } \\
\mu \mathrm{g} / \mathrm{g}\end{array}$ & $\begin{array}{c}\text { Magnesium } \\
\mu \mathrm{g} / \mathrm{g}\end{array}$ & $\begin{array}{c}\text { Sodium } \\
\mu \mathrm{g} / \mathrm{g}\end{array}$ \\
\hline \hline C3393-57.5 & 58.0 & $\mathrm{H} 2$ & $8.76 \mathrm{E}+00$ & $1.02 \mathrm{E}+03$ & $5.62 \mathrm{E}+01$ & $1.20 \mathrm{E}+02$ & $1.82 \mathrm{E}+01$ \\
\hline C3393-57.5 Dup & 58.0 & $\mathrm{H} 2$ & $8.35 \mathrm{E}+00$ & $1.01 \mathrm{E}+03$ & $5.83 \mathrm{E}+01$ & $1.21 \mathrm{E}+02$ & $1.83 \mathrm{E}+01$ \\
\hline C3393-86 & 86.5 & $\mathrm{H} 2$ & $1.07 \mathrm{E}+01$ & $1.23 \mathrm{E}+03$ & $6.15 \mathrm{E}+01$ & $1.15 \mathrm{E}+02$ & $3.63 \mathrm{E}+01$ \\
\hline C3393-86 Dup & 86.5 & $\mathrm{H} 2$ & $1.02 \mathrm{E}+01$ & $1.21 \mathrm{E}+03$ & $6.00 \mathrm{E}+01$ & $1.12 \mathrm{E}+02$ & $3.53 \mathrm{E}+01$ \\
\hline C3393-108.5 & 109.0 & $\mathrm{H} 2$ & $1.39 \mathrm{E}+01$ & $1.24 \mathrm{E}+03$ & $4.33 \mathrm{E}+01$ & $9.45 \mathrm{E}+01$ & $4.30 \mathrm{E}+01$ \\
\hline C3393-108.5 Dup & 109.0 & $\mathrm{H} 2$ & $1.51 \mathrm{E}+01$ & $1.23 \mathrm{E}+03$ & $4.46 \mathrm{E}+01$ & $9.55 \mathrm{E}+01$ & $4.32 \mathrm{E}+01$ \\
\hline C3393-126 & 126.5 & $\mathrm{H} 2$ & $1.43 \mathrm{E}+01$ & $1.30 \mathrm{E}+03$ & $6.30 \mathrm{E}+01$ & $1.19 \mathrm{E}+02$ & $4.09 \mathrm{E}+01$ \\
\hline C3393-126.5 Dup & 126.5 & H2 & $1.49 \mathrm{E}+01$ & $1.30 \mathrm{E}+03$ & $6.54 \mathrm{E}+01$ & $1.21 \mathrm{E}+02$ & $4.25 \mathrm{E}+01$ \\
\hline C3393-141 & 141.5 & CCU & $2.89 \mathrm{E}+01$ & $1.42 \mathrm{E}+03$ & $9.86 \mathrm{E}+01$ & $2.35 \mathrm{E}+02$ & $5.31 \mathrm{E}+01$ \\
\hline C3393-141 Dup & 141.5 & CCU & $2.81 \mathrm{E}+01$ & $1.45 \mathrm{E}+03$ & $9.83 \mathrm{E}+01$ & $2.33 \mathrm{E}+02$ & $5.27 \mathrm{E}+01$ \\
\hline
\end{tabular}

Table 4.18. Cation Exchange Capacity (CEC) of Sediments from the Background Borehole

\begin{tabular}{|l|c|c|c|}
\hline Sample ID & $\begin{array}{c}\text { Mid Depth } \\
\text { (ft bgs) }\end{array}$ & $\begin{array}{c}\text { Stratigraphic } \\
\text { Unit }\end{array}$ & $\begin{array}{c}\text { CEC } \\
\text { (meq/100g) }\end{array}$ \\
\hline C3393-57.5 & 58.0 & H2 & $6.34 \mathrm{E}+00$ \\
\hline C3393-57.5 Dup & 58.0 & H2 & $6.33 \mathrm{E}+00$ \\
\hline C3393-86 & 86.5 & H2 & $7.46 \mathrm{E}+00$ \\
\hline C3393-86 Dup & 86.5 & H2 & $7.33 \mathrm{E}+00$ \\
\hline C3393-108.5 & 109.0 & H2 & $7.31 \mathrm{E}+00$ \\
\hline C3393-108.5 Dup & 109.0 & H2 & $7.28 \mathrm{E}+00$ \\
\hline C3393-126 & 126.5 & H2 & $8.05 \mathrm{E}+00$ \\
\hline C3393-126.5 Dup & 126.5 & H2 & $8.07 \mathrm{E}+00$ \\
\hline C3393-141 & 141.5 & CCU & $9.77 \mathrm{E}+00$ \\
\hline C3393-141 Dup & 141.5 & CCU & $9.91 \mathrm{E}+00$ \\
\hline
\end{tabular}

\subsection{Vadose Zone Sediment from the U Tank Farm Direct Push Samples}

\subsubsection{Moisture Content}

The moisture contents of the 65 core liners and 24 grab samples collected from the U Farm direct push holes are presented as a function of depth in Table 4.19. The depths at which the direct push samples were collected were based on neutron-moisture measurements performed in the field. The intent was to retrieve vadose zone sediment from regions of elevated moisture. As seen in Table 4.19, of the ten direct push holes emplaced during the U Tank Farm campaign, five contained very moist sediment. Specifically, sediments from at least one liner sample retrieved from push holes C5598, C5600, C5602, C5606, and C5608 had moisture contents in excess of $15 \mathrm{wt} \%$. The highest sediment moisture content measured in the U Farm direct push samples at 19.8\% (C5602) was consistent with the peak moisture content (18.3\%) measured in the Hanford formation $\mathrm{H} 2$ unit in the background borehole (C3393). Therefore, no correlation can be made between moisture content and the potential presence of tank waste in the sediments. 
Table 4.19. Gravimetric Moisture Content of Samples Obtained from the U Tank Farm Direct Push Holes

\begin{tabular}{|c|c|c|c|c|}
\hline Sample ID & Probe Hole ID & $\begin{array}{l}\text { Mid-Depth } \\
\text { (ft bgs) }\end{array}$ & Lithology & Moisture (\%) \\
\hline "B1NDW3C & 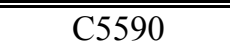 & 995.8 & 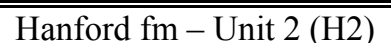 & $8.59 \%$ \\
\hline B1NDW3B & C5590 & 96.3 & Hanford fm - Unit $2(\mathrm{H} 2)$ & $7.96 \%$ \\
\hline B1NDW3A & C5590 & 96.8 & Hanford fm - Unit $2(\mathrm{H} 2)$ & $11.0 \%$ \\
\hline B1NDW3 & C5590 & 97.3 & Hanford fm - Unit 2 (H2) & $7.57 \%$ \\
\hline B1NHV0C & C5592 & 61.8 & Hanford fm - Unit $2(\mathrm{H} 2)$ & $5.96 \%$ \\
\hline B1NHV0B & C5592 & 62.3 & Hanford fm - Unit $2(\mathrm{H} 2)$ & $13.1 \%$ \\
\hline B1NHV0A & C5592 & 62.8 & Hanford fm - Unit 2 (H2) & $7.87 \%$ \\
\hline B1NHV0 & C5592 & 63.3 & Hanford fm - Unit 2 (H2) & $4.44 \%$ \\
\hline B1NDW4 & C5594A & 57.3 & Hanford fm - Unit 1 (H1) & $1.51 \%$ \\
\hline B1NTC6C & C5598 & 49.8 & Hanford fm - Unit 2 (H2) & $6.73 \%$ \\
\hline B1NTC6B & C5598 & 50.3 & Hanford fm - Unit 2 (H2) & $10.5 \%$ \\
\hline B1NTC6A & C5598 & 50.8 & Hanford fm - Unit 2 (H2) & $13.2 \%$ \\
\hline B1NTC6 & C5598 & 51.3 & Hanford fm - Unit 2 (H2) & $7.25 \%$ \\
\hline B1NTC7C & C5598 & 59.3 & Hanford fm - Unit 2 (H2) & $5.09 \%$ \\
\hline B1NTC7B & C5598 & 59.8 & Hanford fm - Unit $2(\mathrm{H} 2)$ & $8.21 \%$ \\
\hline B1NTC7A & $\mathrm{C} 5598$ & 60.3 & Hanford fm - Unit $2(\mathrm{H} 2)$ & $8.02 \%$ \\
\hline B1NTC7 & C5598 & 60.8 & Hanford fm - Unit 2 (H2) & $2.13 \%$ \\
\hline B1NTC8C & C5598 & 81.8 & Hanford fm - Unit 2 (H2) & $4.35 \%$ \\
\hline B1NTC8B & C5598 & 82.3 & Hanford fm - Unit $2(\mathrm{H} 2)$ & $16.1 \%$ \\
\hline B1NTC8A & C5598 & 82.8 & Hanford fm - Unit 2 (H2) & $8.97 \%$ \\
\hline B1NTC8 & C5598 & 83.3 & Hanford fm - Unit $2(\mathrm{H} 2)$ & $4.79 \%$ \\
\hline B1NTC9C & C5596 & 50.3 & Hanford fm - Unit $1(\mathrm{H} 1)$ & $4.41 \%$ \\
\hline B1NTC9B & C5596 & 50.8 & Hanford fm - Unit $2(\mathrm{H} 2)$ & $6.04 \%$ \\
\hline B1NTC9A & C5596 & 51.3 & Hanford fm - Unit 2 (H2) & $5.23 \%$ \\
\hline B1NTC9 & C5596 & 51.8 & Hanford fm - Unit 2 (H2) & $11.5 \%$ \\
\hline B1NTD0C & C5596 & 60.8 & Hanford fm - Unit 2 (H2) & $4.22 \%$ \\
\hline B1NTD0B & C5596 & 61.3 & Hanford fm - Unit $2(\mathrm{H} 2)$ & $7.79 \%$ \\
\hline B1NTD0A & C5596 & 61.8 & Hanford fm - Unit 2 (H2) & $6.07 \%$ \\
\hline B1NTD0 & $\mathrm{C} 5596$ & 62.3 & Hanford fm - Unit $2(\mathrm{H} 2)$ & $4.15 \%$ \\
\hline B1NTD1C & $\mathrm{C} 5596$ & 77.3 & Hanford fm - Unit $2(\mathrm{H} 2)$ & $7.45 \%$ \\
\hline B1NTD1B & C5596 & 77.8 & Hanford fm - Unit 2 (H2) & $6.37 \%$ \\
\hline B1NTD1A & C5596 & 78.3 & Hanford fm - Unit $2(\mathrm{H} 2)$ & $6.63 \%$ \\
\hline B1NTD1 & $\mathrm{C} 5596$ & 78.8 & Hanford fm - Unit $2(\mathrm{H} 2)$ & $4.65 \%$ \\
\hline B1NTD2C & $\mathrm{C} 5596$ & 82.3 & Hanford fm - Unit $2(\mathrm{H} 2)$ & $4.47 \%$ \\
\hline B1NTD2B & C5596 & 82.8 & Hanford fm - Unit 2 (H2) & $3.16 \%$ \\
\hline B1NTD2A & C5596 & 83.3 & Hanford fm - Unit $2(\mathrm{H} 2)$ & $2.94 \%$ \\
\hline B1NTD2 & C5596 & 83.8 & Hanford fm - Unit $2(\mathrm{H} 2)$ & $2.23 \%$ \\
\hline B1NTD3C & $\mathrm{C} 5600$ & 49.8 & Hanford fm - Unit $2(\mathrm{H} 2)$ & $10.3 \%$ \\
\hline B1NTD3B & $\mathrm{C} 5600$ & 50.3 & Hanford fm - Unit $2(\mathrm{H} 2)$ & $12.0 \%$ \\
\hline B1NTD3A & $\mathrm{C} 5600$ & 50.8 & Hanford fm - Unit $2(\mathrm{H} 2)$ & $9.85 \%$ \\
\hline B1NTD3 & C5600 & 51.3 & Hanford fm - Unit 2 (H2) & $7.31 \%$ \\
\hline B1NTD4C & $\mathrm{C} 5600$ & 59.8 & Hanford fm - Unit $2(\mathrm{H} 2)$ & $5.99 \%$ \\
\hline B1NTD4B & C5600 & 60.3 & Hanford fm - Unit $2(\mathrm{H} 2)$ & $4.84 \%$ \\
\hline B1NTD4A & C5600 & 60.8 & Hanford fm - Unit $2(\mathrm{H} 2)$ & $8.80 \%$ \\
\hline B1NTD4 & $\mathrm{C} 5600$ & 61.3 & Hanford fm - Unit $2(\mathrm{H} 2)$ & $7.64 \%$ \\
\hline B1NTD5C & $\mathrm{C} 5600$ & 81.8 & Hanford fm - Unit 2 (H2) & $9.97 \%$ \\
\hline B1NTD5B & $\mathrm{C} 5600$ & 82.3 & Hanford fm - Unit $2(\mathrm{H} 2)$ & $15.8 \%$ \\
\hline
\end{tabular}




\begin{tabular}{|c|c|c|c|c|}
\hline Sample ID & Probe Hole ID & $\begin{array}{l}\text { Mid-Depth } \\
\text { (ft bgs) }\end{array}$ & Lithology & Moisture (\%) \\
\hline B1NTD5A & C5600 & 82.8 & Hanford fm - Unit 2 (H2) & $5.57 \%$ \\
\hline B1NTD5 & C5600 & 83.3 & Hanford fm - Unit 2 (H2) & $4.31 \%$ \\
\hline B1P1K6C & C5600 & 88.3 & Hanford fm - Unit 2 (H2) & $5.49 \%$ \\
\hline B1P1K6B & $\mathrm{C} 5600$ & 88.8 & Hanford fm - Unit 2 (H2) & $3.98 \%$ \\
\hline B1P1K6A & C5600 & 89.3 & Hanford fm - Unit $2(\mathrm{H} 2)$ & $7.93 \%$ \\
\hline B1P1K6 & $\mathrm{C} 5600$ & 89.8 & Hanford fm - Unit 2 (H2) & $6.96 \%$ \\
\hline B1P3H0C & C5602 & 51.3 & Hanford fm - Unit $2(\mathrm{H} 2)$ & $18.2 \%$ \\
\hline B1P3H0B & C5602 & 51.8 & Hanford fm - Unit $2(\mathrm{H} 2)$ & $7.53 \%$ \\
\hline B1P3H0A & C5602 & 52.3 & Hanford fm - Unit 2 (H2) & $2.77 \%$ \\
\hline B1P3H0 & C5602 & 52.8 & Hanford fm - Unit 2 (H2) & $3.16 \%$ \\
\hline B1P3H1C & $\mathrm{C} 5602$ & 67.3 & Hanford fm - Unit 2 (H2) & $5.85 \%$ \\
\hline B1P3H1B & C5602 & 67.8 & Hanford fm - Unit $2(\mathrm{H} 2)$ & $3.82 \%$ \\
\hline B1P3H1A & C5602 & 68.3 & Hanford fm - Unit 2 (H2) & $9.63 \%$ \\
\hline $\mathrm{B} 1 \mathrm{P} 3 \mathrm{H} 1$ & $\mathrm{C} 5602$ & 68.8 & Hanford fm - Unit $2(\mathrm{H} 2)$ & $11.7 \%$ \\
\hline B1P3H2C & C5602 & 82.3 & Hanford fm - Unit 2 (H2) & $19.8 \%$ \\
\hline B1P3H2B & C5602 & 82.8 & Hanford fm - Unit $2(\mathrm{H} 2)$ & $9.53 \%$ \\
\hline B1P3H2A & C5602 & 83.3 & Hanford fm - Unit 2 (H2) & $4.43 \%$ \\
\hline B1P3H2 & C5602 & 83.8 & Hanford fm - Unit $2(\mathrm{H} 2)$ & $3.64 \%$ \\
\hline B1PBB0C & C5602 & 91.3 & Hanford fm - Unit 2 (H2) & $16.5 \%$ \\
\hline B1PBB0B & C5602 & 91.8 & Hanford fm - Unit $2(\mathrm{H} 2)$ & $7.82 \%$ \\
\hline B1PBB0A & C5602 & 92.3 & Hanford fm - Unit 2 (H2) & $6.13 \%$ \\
\hline B1PBB0 & $\mathrm{C} 5602$ & 92.8 & Hanford fm - Unit $2(\mathrm{H} 2)$ & $3.75 \%$ \\
\hline B1P3F9C & C5604 & 50.3 & Hanford fm - Unit 2 (H2) & $6.05 \%$ \\
\hline B1P3F9B & C5604 & 50.8 & Hanford fm - Unit 2 (H2) & $13.6 \%$ \\
\hline B1P3F9A & C5604 & 51.3 & Hanford fm - Unit 2 (H2) & $14.9 \%$ \\
\hline B1P3F9 & C5604 & 51.8 & Hanford fm - Unit $2(\mathrm{H} 2)$ & $4.10 \%$ \\
\hline B1PBB1C & C5606 & 51.3 & Hanford fm - Unit 2 (H2) & $11.1 \%$ \\
\hline B1PBB1B & C5606 & 51.8 & Hanford fm - Unit $2(\mathrm{H} 2)$ & $17.5 \%$ \\
\hline B1PBB1A & C5606 & 52.3 & Hanford fm - Unit 2 (H2) & $19.4 \%$ \\
\hline B1PBB1 & C5606 & 52.8 & Hanford fm - Unit $2(\mathrm{H} 2)$ & $7.52 \%$ \\
\hline B1PK51C & C5608 & 63.8 & Hanford fm - Unit 2 (H2) & $6.82 \%$ \\
\hline B1PK51B & C5608 & 64.3 & Hanford fm - Unit $2(\mathrm{H} 2)$ & $17.9 \%$ \\
\hline B1PK51A & C5608 & 64.8 & Hanford fm - Unit 2 (H2) & $7.31 \%$ \\
\hline B1PK51 & C5608 & 65.3 & Hanford fm - Unit 2 (H2) & $5.05 \%$ \\
\hline B1PK52C & C5608 & 85.3 & Hanford fm - Unit 2 (H2) & $13.5 \%$ \\
\hline B1PK52B & C5608 & 85.8 & Hanford fm - Unit 2 (H2) & $19.5 \%$ \\
\hline B1PK52A & C5608 & 86.3 & Hanford fm - Unit $2(\mathrm{H} 2)$ & $17.3 \%$ \\
\hline B1PK52 & C5608 & 86.8 & Hanford fm - Unit 2 (H2) & $11.1 \%$ \\
\hline B1PK53C & C5608 & 97.3 & Hanford fm - Unit 2 (H2) & $11.6 \%$ \\
\hline B1PK53B & C5608 & 97.8 & Hanford fm - Unit 2 (H2) & $12.4 \%$ \\
\hline B1PK53A & C5608 & 98.3 & Hanford fm - Unit $2(\mathrm{H} 2)$ & $5.12 \%$ \\
\hline B1PK53 & C5608 & 98.8 & Hanford fm - Unit 2 (H2) & $4.52 \%$ \\
\hline
\end{tabular}

\subsubsection{1:1 Sediment:Water Extracts}

The samples from the U Tank Farm direct push campaign were characterized by performing 1:1 sediment:water extracts. The following tables present the mass of a given constituent leached per gram of 
sediment as measured in the water extracts. Other tables show dilution-corrected values that represent concentrations in vadose zone pore water. As discussed in several other Vadose Zone Characterization Project reports, the dilution-corrected 1:1 sediment:water extracts are a reasonable estimate of the actual vadose zone pore water (see Serne et al. 2002a, 2002b, 2002c, 2002e, 2002f).

\subsubsection{1 $\mathrm{pH}$ and Electrical Conductivity}

The 1:1 sediment:water extract $\mathrm{pH}$ and $\mathrm{EC}$ data for the $\mathrm{U}$ Tank Farm direct push core samples are shown in Table 4.20. The $\mathrm{pH}$ is tabulated as measured in the 1:1 sediment:water extracts but the EC is corrected for dilution and tabulated as if it was actual pore water. Nearly all of the extract samples tested had $\mathrm{pH}$ values in the normal range for Hanford formation sediments (between 7.5 and 8.0). However, one of the push holes, C5608, contained sediment with an elevated pH. Specifically, all six of the cores analyzed from push hole $\mathrm{C} 5608$ had soil $\mathrm{pH}$ values in excess of 9.0. The peak soil $\mathrm{pH}$, at 10.3, was measured in a sample retrieved from approximately $86 \mathrm{ft}$ bgs (B1PK52A). Previous characterization reports have shown that regions of elevated soil $\mathrm{pH}$ are considered to be good indicators of the location of the original leak event or very near-field close to the initial tank waste entry zone (see Serne et al. 2002a, 2002b, 2002c, 2002e, 2002f). Therefore, it appears that direct push hole C5608, emplaced near tank 241$\mathrm{U}-110$, was located in close proximity to the location of the leak.

The pore water-corrected EC data for all of the samples from the U Tank Farm (except those from push hole C5608) were low, with a range of 1.25 to $6.39 \mathrm{mS} / \mathrm{cm}$. Conversely, samples collected from push hole $\mathrm{C} 5608$ had porewater-corrected conductivities ranging from 5.13 to $22.8 \mathrm{mS} / \mathrm{cm}$. The peak porewater-corrected conductivity $(22.8 \mathrm{mS} / \mathrm{cm})$ was measured in the deepest sample analyzed from push hole C5608. For comparison, the background borehole (C3393) contained samples with porewatercorrected conductivities ranging from 0.978 to $10.5 \mathrm{mS} / \mathrm{cm}$. Therefore, with the exception of samples from push hole $\mathrm{C} 5608$, the U Tank Farm direct push samples appeared to be dilute with respect to dissolved salt content and were comparable to dissolved salts loads measured in samples from the background borehole.

Table 4.20. $\mathrm{pH}$ for 1:1 Sediment:Water Extracts and Dilution-Corrected EC Values from U Tank Farm Samples

\begin{tabular}{||l|c|c|c|c||}
\hline $\begin{array}{c}\text { Sample } \\
\text { ID }\end{array}$ & $\begin{array}{c}\text { Probe Hole } \\
\text { ID }\end{array}$ & $\begin{array}{c}\text { Mid-Depth } \\
\mathrm{ft} \text { bgs }\end{array}$ & $\mathrm{pH}$ & $\begin{array}{c}\text { Conductivity } \\
\text { (mS/cm) }\end{array}$ \\
\hline \hline B1NDW3B & C5590 & 96.3 & 7.13 & $1.93 \mathrm{E}+00$ \\
\hline B1NDW3A & C5590 & 96.8 & 7.11 & $1.55 \mathrm{E}+00$ \\
\hline B1NDW3A DUP & C5590 & 96.8 & 7.10 & $1.63 \mathrm{E}+00$ \\
\hline B1NHV0B & C5592 & 62.3 & 7.35 & $1.39 \mathrm{E}+00$ \\
\hline B1NHV0A & C5592 & 62.8 & 7.35 & $1.99 \mathrm{E}+00$ \\
\hline B1NTC6B & C5598 & 50.3 & 7.27 & $1.75 \mathrm{E}+00$ \\
\hline B1NTC6A & C5598 & 50.8 & 7.59 & $1.62 \mathrm{E}+00$ \\
\hline B1NTC7B & C5598 & 59.8 & 7.55 & $2.36 \mathrm{E}+00$ \\
\hline B1NTC7A & C5598 & 60.3 & 7.59 & $2.26 \mathrm{E}+00$ \\
\hline B1NTC8B & C5598 & 82.3 & 7.52 & $1.78 \mathrm{E}+00$ \\
\hline B1NTC8B DUP & C5598 & 82.3 & 7.65 & $1.76 \mathrm{E}+00$ \\
\hline B1NTC8A & C5598 & 82.8 & 7.71 & $2.64 \mathrm{E}+00$ \\
\hline B1NTC9B & C5596 & 50.8 & 7.74 & $2.75 \mathrm{E}+00$ \\
\hline B1NTC9A & C5596 & 51.3 & 7.73 & $3.01 \mathrm{E}+00$ \\
\hline
\end{tabular}




\begin{tabular}{|c|c|c|c|c|}
\hline $\begin{array}{l}\text { Sample } \\
\text { ID }\end{array}$ & $\begin{array}{l}\text { Probe Hole } \\
\text { ID }\end{array}$ & $\begin{array}{l}\text { Mid-Depth } \\
\text { ft bgs }\end{array}$ & $\mathrm{pH}$ & $\begin{array}{c}\text { Conductivity } \\
(\mathrm{mS} / \mathrm{cm})\end{array}$ \\
\hline B1NTD0B & "C5596 & 61.3 & 7.72 & $2.41 \mathrm{E}+00$ \\
\hline$\overline{B 1 N T D 0 A}$ & C5596 & 61.8 & 7.20 & $3.11 \mathrm{E}+00$ \\
\hline B1NTD1B & C5596 & 77.8 & 7.30 & $3.69 \mathrm{E}+00$ \\
\hline B1NTD1B DUP & C5596 & 77.8 & 7.42 & $3.82 \mathrm{E}+00$ \\
\hline B1NTD1A & C5596 & 78.3 & 7.47 & $3.65 \mathrm{E}+00$ \\
\hline B1NTD2B & C5596 & 82.8 & 7.44 & $6.21 \mathrm{E}+00$ \\
\hline B1NTD2A & C5596 & 83.3 & 7.52 & $6.39 \mathrm{E}+00$ \\
\hline B1NTD3B & C5600 & 50.3 & 7.56 & $1.54 \mathrm{E}+00$ \\
\hline B1NTD3A & C5600 & 50.8 & 7.60 & $1.84 \mathrm{E}+00$ \\
\hline B1NTD4B & C5600 & 60.3 & 7.30 & $2.88 \mathrm{E}+00$ \\
\hline B1NTD4A & C5600 & 60.8 & 7.41 & $1.69 \mathrm{E}+00$ \\
\hline B1NTD5B & C5600 & 82.3 & 7.57 & $1.80 \mathrm{E}+00$ \\
\hline B1NTD5A & C5600 & 82.8 & 7.36 & $3.32 \mathrm{E}+00$ \\
\hline B1P1K6B & C5600 & 88.8 & 7.48 & $4.72 \mathrm{E}+00$ \\
\hline B1P1K6A & $\mathrm{C} 5600$ & 89.3 & 7.52 & $3.01 \mathrm{E}+00$ \\
\hline B1P3F9B & C5604 & 50.8 & 7.38 & $1.56 \mathrm{E}+00$ \\
\hline B1P3F9A & C5604 & 51.3 & 7.50 & $1.25 \mathrm{E}+00$ \\
\hline B1P3H0B & C5602 & 51.8 & 7.59 & $2.11 \mathrm{E}+00$ \\
\hline B1P3H0A & C5602 & 52.3 & 7.48 & $4.62 \mathrm{E}+00$ \\
\hline B1P3H1B & C5602 & 67.8 & 7.49 & $3.57 \mathrm{E}+00$ \\
\hline B1P3H1A & C5602 & 68.3 & 7.54 & $1.83 \mathrm{E}+00$ \\
\hline B1P3H2B & $\mathrm{C} 5602$ & 82.8 & 7.57 & $2.30 \mathrm{E}+00$ \\
\hline B1P3H2A & C5602 & 83.3 & 7.59 & $3.23 \mathrm{E}+00$ \\
\hline B1PBB0B & C5602 & 91.8 & 7.44 & $2.93 \mathrm{E}+00$ \\
\hline B1PBB0A & C5602 & 92.3 & 7.30 & $2.97 \mathrm{E}+00$ \\
\hline B1PBB1B & C5606 & 51.8 & 7.25 & $1.35 \mathrm{E}+00$ \\
\hline B1PBB1A & C5606 & 52.3 & 7.45 & $1.32 \mathrm{E}+00$ \\
\hline B1PBB1A DUP & C5606 & 52.3 & 7.37 & $1.28 \mathrm{E}+00$ \\
\hline B1PK51B & C5608 & 64.3 & 9.24 & $5.13 \mathrm{E}+00$ \\
\hline B1PK51A & C5608 & 64.8 & 9.45 & $9.46 \mathrm{E}+00$ \\
\hline B1PK52B & C5608 & 85.8 & 9.91 & $1.67 E+01$ \\
\hline B1PK52A & C5608 & 86.3 & 10.3 & $1.78 \mathrm{E}+01$ \\
\hline B1PK53B & C5608 & 97.8 & 9.33 & $1.47 \mathrm{E}+01$ \\
\hline B1PK53A & C5608 & 98.3 & 9.52 & $2.28 \mathrm{E}+01$ \\
\hline
\end{tabular}

\subsubsection{Composition of the 1:1 Sediment:Water Extracts from the U Tank Farm Core Samples}

The water extract values for the major anions, cations, and several trace constituents are discussed in this section. The anion data are tabulated in Table 4.21 in units of mass per gram of dry sediment. Consistent with our previous findings with the ph and EC data, the only samples that were significantly elevated with respect to dissolved anions were those collected from push hole C5608. Specifically, the two deepest sample strings collected from push hole C5608 (85 ft bgs and $98 \mathrm{ft}$ bgs, respectively) contained elevated water-extractable fluoride, nitrate, and phosphate. The peak fluoride concentration 
measured in the C5608 samples, at $18.1 \mu \mathrm{g} / \mathrm{g}$, was more than an order of magnitude greater than the peak concentration measured in the background borehole (C3393). The peak water-extractable nitrate (578 $\mu \mathrm{g} / \mathrm{g}$ ) measured in the C5608 push hole was nearly two orders of magnitude greater than that measured in borehole C3393 $(8.54 \mu \mathrm{g} / \mathrm{g}$ ). Finally, the peak phosphate concentration found in push hole C5608 (2.66 $\mu \mathrm{g} / \mathrm{g}$ ) was approximately five times greater than the peak phosphate concentration in the background borehole $(0.57 \mu \mathrm{g} / \mathrm{g})$.

The water-extractable major cations in the U Tank Farm direct push sediments are tabulated in Table 4.22 in units of mass per gram of sediment on a dry weight basis. Consistent with the anion data tabulated in Table 4.21, the only samples that contained grossly elevated water extractable major cations were those from push hole C5608. All six of the liner samples analyzed from push hole C5608 contained nearly an order of magnitude or more water-extractable sodium than those measured from borehole C3393. Coincident with the elevated sodium, water-extractable concentrations of calcium, potassium, and magnesium were negligible to non-quantifiable. It is apparent that for these samples, sodium has driven the divalent cations off the exchange sites. Based on this data, it is clear that a sodium-rich waste stream has migrated to at least $96 \mathrm{ft}$ bgs adjacent to tank 241-U-110.

Table 4.21. Water-Extractable Anions in the U Tank Farm Core Samples ( $\mu \mathrm{g} / \mathrm{g}$ dry sediment)

\begin{tabular}{|c|c|c|c|c|c|c|c|}
\hline $\begin{array}{l}\text { Sample } \\
\text { ID }\end{array}$ & $\begin{array}{l}\text { Probe Hole } \\
\text { ID }\end{array}$ & $\begin{array}{l}\text { Mid-Depth } \\
\text { ft bgs }\end{array}$ & $\begin{array}{c}\text { Fluoride } \\
\mu \mathrm{g} / \mathrm{g}\end{array}$ & $\begin{array}{c}\text { Chloride } \\
\mu \mathrm{g} / \mathrm{g}\end{array}$ & $\begin{array}{c}\text { Nitrate } \\
\mu \mathrm{g} / \mathrm{g}\end{array}$ & $\begin{array}{c}\begin{array}{c}\text { Sulfate } \\
\mu \mathrm{g} / \mathrm{g}\end{array}\end{array}$ & $\begin{array}{c}\text { Phosphate } \\
\mu \mathrm{g} / \mathrm{g}\end{array}$ \\
\hline B1NDW3B & C5590 & 96.3 & $2.90 \mathrm{E}-01$ & $9.9 .07 \mathrm{E}+00$ & $101.75 \mathrm{E}+00$ & $7.82 \mathrm{E}+00$ & 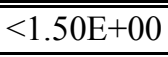 \\
\hline B1NDW3A & C5590 & 96.8 & $3.19 \mathrm{E}-01$ & $1.59 \mathrm{E}+01$ & $2.59 \mathrm{E}+00$ & $9.84 \mathrm{E}+00$ & $<1.50 \mathrm{E}+00$ \\
\hline B1NDW3A DUP & C5590 & 96.8 & $3.36 \mathrm{E}-01$ & $1.59 \mathrm{E}+01$ & $2.83 \mathrm{E}+00$ & $1.02 \mathrm{E}+01$ & $<1.50 \mathrm{E}+00$ \\
\hline B1NHV0B & C5592 & 62.3 & $6.68 \mathrm{E}-01$ & $7.62 \mathrm{E}-01$ & $6.80 \mathrm{E}+00$ & $1.07 \mathrm{E}+01$ & $<1.50 \mathrm{E}+00$ \\
\hline B1NHV0A & C5592 & 62.8 & $3.42 \mathrm{E}-01$ & $5.98 \mathrm{E}-01$ & $5.05 \mathrm{E}+00$ & $9.12 \mathrm{E}+00$ & $<1.50 \mathrm{E}+00$ \\
\hline B1NTC6B & C5598 & 50.3 & $1.07 \mathrm{E}+00$ & $1.56 \mathrm{E}+00$ & $8.09 \mathrm{E}+00$ & $3.99 \mathrm{E}+00$ & $<1.51 \mathrm{E}+00$ \\
\hline B1NTC6A & C5598 & 50.8 & $8.15 \mathrm{E}-01$ & $1.92 \mathrm{E}+00$ & $1.56 \mathrm{E}+01$ & $9.26 \mathrm{E}+00$ & $<1.50 \mathrm{E}+00$ \\
\hline B1NTC7B & C5598 & 59.8 & 7.49E-01 & $1.02 \mathrm{E}+00$ & $4.59 \mathrm{E}+00$ & $1.43 \mathrm{E}+01$ & $<1.50 \mathrm{E}+00$ \\
\hline B1NTC7A & C5598 & 60.3 & $6.64 \mathrm{E}-01$ & $1.16 \mathrm{E}+00$ & $3.95 \mathrm{E}+00$ & $1.21 \mathrm{E}+01$ & $<1.50 \mathrm{E}+00$ \\
\hline B1NTC8B & C5598 & 82.3 & $7.88 \mathrm{E}-01$ & $9.04 \mathrm{E}+00$ & $2.76 \mathrm{E}+01$ & $2.44 \mathrm{E}+01$ & $<1.50 \mathrm{E}+00$ \\
\hline B1NTC8B DUP & C5598 & 82.3 & $8.25 \mathrm{E}-01$ & $1.01 \mathrm{E}+01$ & $2.82 \mathrm{E}+01$ & $2.54 \mathrm{E}+01$ & $<1.50 \mathrm{E}+00$ \\
\hline B1NTC8A & C5598 & 82.8 & NR & $6.32 \mathrm{E}+00$ & $1.04 \mathrm{E}+01$ & $2.09 \mathrm{E}+01$ & $<1.50 \mathrm{E}+00$ \\
\hline B1NTC9B & C5596 & 50.8 & NR & $7.11 \mathrm{E}-01$ & $4.66 \mathrm{E}+00$ & $2.48 \mathrm{E}+00$ & $<1.50 \mathrm{E}+00$ \\
\hline B1NTC9A & C5596 & 51.3 & NR & $6.57 \mathrm{E}-01$ & $3.74 \mathrm{E}+00$ & $1.90 \mathrm{E}+00$ & $<1.53 \mathrm{E}+00$ \\
\hline B1NTD0B & C5596 & 61.3 & NR & $1.06 \mathrm{E}+00$ & $2.93 \mathrm{E}+00$ & $1.63 \mathrm{E}+01$ & $<1.50 \mathrm{E}+00$ \\
\hline B1NTD0A & C5596 & 61.8 & $8.73 \mathrm{E}-01$ & $1.19 \mathrm{E}+00$ & $<1.02 \mathrm{E}+00$ & $1.53 \mathrm{E}+01$ & $<1.53 \mathrm{E}+00$ \\
\hline B1NTD1B & C5596 & 77.8 & $6.58 \mathrm{E}-01$ & $1.80 \mathrm{E}+00$ & $1.96 \mathrm{E}+01$ & $2.14 \mathrm{E}+01$ & $<1.67 \mathrm{E}+00$ \\
\hline B1NTD1B DUP & C5596 & 77.8 & $6.47 \mathrm{E}-01$ & $2.06 \mathrm{E}+00$ & $2.30 \mathrm{E}+01$ & $2.42 \mathrm{E}+01$ & $<1.50 \mathrm{E}+00$ \\
\hline B1NTD1A & C5596 & 78.3 & $6.45 \mathrm{E}-01$ & $2.45 \mathrm{E}+00$ & $2.02 \mathrm{E}+01$ & $2.78 \mathrm{E}+01$ & $<1.50 \mathrm{E}+00$ \\
\hline B1NTD2B & C5596 & 82.8 & NR & $4.59 \mathrm{E}+00$ & $1.47 \mathrm{E}+01$ & $9.82 \mathrm{E}+00$ & $<1.50 \mathrm{E}+00$ \\
\hline B1NTD2A & C5596 & 83.3 & NR & $4.42 \mathrm{E}+00$ & $1.30 \mathrm{E}+01$ & $8.93 \mathrm{E}+00$ & $<1.50 \mathrm{E}+00$ \\
\hline B1NTD3B & C5600 & 50.3 & NR & $8.14 \mathrm{E}-01$ & $1.82 \mathrm{E}+01$ & $7.84 \mathrm{E}+00$ & $<1.50 \mathrm{E}+00$ \\
\hline B1NTD3A & C5600 & 50.8 & NR & $1.69 \mathrm{E}+00$ & $1.23 \mathrm{E}+01$ & $6.74 \mathrm{E}+00$ & $<1.50 \mathrm{E}+00$ \\
\hline B1NTD4B & C5600 & 60.3 & $6.55 \mathrm{E}-01$ & $5.60 \mathrm{E}-01$ & $1.45 \mathrm{E}+00$ & $5.54 \mathrm{E}+00$ & $<1.50 \mathrm{E}+00$ \\
\hline B1NTD4A & C5600 & 60.8 & $6.57 \mathrm{E}-01$ & $<5.00 \mathrm{E}-01$ & $2.53 \mathrm{E}+00$ & $7.31 \mathrm{E}+00$ & $<1.50 \mathrm{E}+00$ \\
\hline B1NTD5B & C5600 & 82.3 & $3.81 \mathrm{E}-01$ & $9.49 \mathrm{E}+00$ & $1.46 \mathrm{E}+01$ & $3.85 \mathrm{E}+01$ & $<1.51 \mathrm{E}+00$ \\
\hline B1NTD5A & C5600 & 82.8 & $8.02 \mathrm{E}-01$ & $3.99 \mathrm{E}+00$ & $<1.01 \mathrm{E}+00$ & $1.71 \mathrm{E}+01$ & $<1.51 \mathrm{E}+00$ \\
\hline
\end{tabular}




\begin{tabular}{|c|c|c|c|c|c|c|c|}
\hline "B1P1K6B & "C5600 & 88.8 & "NR & "7.05E+00 & "5.97E+00 & $=1.21 \mathrm{E}+01$ & 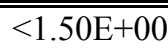 \\
\hline B1P1K6A & C5600 & 89.3 & NR & $1.21 \mathrm{E}+01$ & $9.17 \mathrm{E}+00$ & $1.89 \mathrm{E}+01$ & $<1.50 \mathrm{E}+00$ \\
\hline B1P3F9B & C5604 & 50.8 & NR & $6.00 \mathrm{E}+00$ & $4.03 \mathrm{E}+00$ & $5.67 \mathrm{E}+00$ & $<1.50 \mathrm{E}+00$ \\
\hline B1P3F9A & C5604 & 51.3 & NR & $5.26 \mathrm{E}-01$ & $2.59 \mathrm{E}+00$ & $5.68 \mathrm{E}+00$ & $<1.50 \mathrm{E}+00$ \\
\hline B1P3H0B & C5602 & 51.8 & NR & $1.02 \mathrm{E}+00$ & $4.73 \mathrm{E}+00$ & $4.46 \mathrm{E}+00$ & $<1.50 \mathrm{E}+00$ \\
\hline B1P3H0A & C5602 & 52.3 & NR & $4.94 \mathrm{E}-01$ & $1.30 \mathrm{E}+00$ & $1.70 \mathrm{E}+00$ & $<1.50 \mathrm{E}+00$ \\
\hline B1P3H1B & C5602 & 67.8 & NR & $5.69 \mathrm{E}-01$ & $2.28 \mathrm{E}+00$ & $6.72 \mathrm{E}+00$ & $<1.50 \mathrm{E}+00$ \\
\hline B1P3H1A & C5602 & 68.3 & NR & $8.72 \mathrm{E}-01$ & $5.53 \mathrm{E}+00$ & $1.32 \mathrm{E}+01$ & $<1.50 \mathrm{E}+00$ \\
\hline B1P3H2B & C5602 & 82.8 & NR & $1.39 \mathrm{E}+00$ & $7.49 \mathrm{E}+00$ & $2.40 \mathrm{E}+01$ & $<1.50 \mathrm{E}+00$ \\
\hline B1P3H2A & C5602 & 83.3 & NR & $5.79 \mathrm{E}-01$ & $2.91 \mathrm{E}+00$ & $1.05 \mathrm{E}+01$ & $<1.50 \mathrm{E}+00$ \\
\hline B1PBB0B & C5602 & 91.8 & NR & $2.05 \mathrm{E}+00$ & $2.21 \mathrm{E}+01$ & $2.86 \mathrm{E}+01$ & $<1.50 \mathrm{E}+00$ \\
\hline B1PBB0A & C5602 & 92.3 & NR & $1.60 \mathrm{E}+00$ & $1.04 \mathrm{E}+01$ & $2.08 \mathrm{E}+01$ & $<1.50 \mathrm{E}+00$ \\
\hline B1PBB1B & C5606 & 51.8 & $3.66 \mathrm{E}-01$ & $1.22 \mathrm{E}+00$ & $2.03 \mathrm{E}+01$ & $3.02 \mathrm{E}+01$ & $<1.50 \mathrm{E}+00$ \\
\hline B1PBB1A & C5606 & 52.3 & $4.65 \mathrm{E}-01$ & $2.07 \mathrm{E}+00$ & $2.12 \mathrm{E}+01$ & $3.13 \mathrm{E}+01$ & $<1.51 \mathrm{E}+00$ \\
\hline B1PBB1A DUP & C5606 & 52.3 & 4.49E-01 & $1.92 \mathrm{E}+00$ & $1.97 \mathrm{E}+01$ & $3.02 \mathrm{E}+01$ & $<1.50 \mathrm{E}+00$ \\
\hline B1PK51B & C5608 & 64.3 & $2.44 \mathrm{E}+00$ & $8.34 \mathrm{E}-01$ & $7.96 \mathrm{E}+00$ & $1.37 \mathrm{E}+01$ & $<1.50 \mathrm{E}+00$ \\
\hline B1PK51A & C5608 & 64.8 & $1.52 \mathrm{E}+00$ & $6.59 \mathrm{E}-01$ & $<1.00 \mathrm{E}+00$ & $6.05 \mathrm{E}+00$ & $<1.50 \mathrm{E}+00$ \\
\hline B1PK52B & C5608 & 85.8 & $1.81 E+01$ & $1.05 \mathrm{E}+01$ & $5.78 \mathrm{E}+02$ & $3.32 \mathrm{E}+01$ & $2.55 \mathrm{E}+00$ \\
\hline B1PK52A & C5608 & 86.3 & $1.39 \mathrm{E}+01$ & $8.19 \mathrm{E}+00$ & $4.87 \mathrm{E}+02$ & $2.88 \mathrm{E}+01$ & $2.66 \mathrm{E}+00$ \\
\hline B1PK53B & C5608 & 97.8 & $1.59 \mathrm{E}+01$ & $8.32 \mathrm{E}+00$ & $4.67 \mathrm{E}+02$ & $4.00 \mathrm{E}+01$ & $1.76 \mathrm{E}+00$ \\
\hline B1PK53A & C5608 & 98.3 & $5.33 E+00$ & $3.29 \mathrm{E}+00$ & $1.93 E+02$ & $1.67 \mathrm{E}+01$ & $<1.50 \mathrm{E}+00$ \\
\hline \multicolumn{8}{|c|}{$\begin{array}{l}\text { Bold values denote concentrations elevated above background. } \\
\text { Less-than values indicate the result was below the sample estimated quantification limit (EQL). The sample EQL has been } \\
\text { reported. } \\
\text { NR indicates the information was not reported due to an analytical interference. }\end{array}$} \\
\hline
\end{tabular}

Table 4.22. Water-Extractable Major Cations in the U Tank Farm Direct Push Samples ( $\mu \mathrm{g} / \mathrm{g}$ dry sediment)

\begin{tabular}{||l|c|c|c|c|c|c|c||}
\hline $\begin{array}{c}\text { Sample } \\
\text { ID }\end{array}$ & $\begin{array}{c}\text { Probe Hole } \\
\text { ID }\end{array}$ & $\begin{array}{c}\text { Mid-Depth } \\
\mathrm{ft} \text { bgs }\end{array}$ & $\begin{array}{c}\text { Calcium } \\
\mu \mathrm{g} / \mathrm{g}\end{array}$ & $\begin{array}{c}\text { Potassium } \\
\mu \mathrm{g} / \mathrm{g}\end{array}$ & $\begin{array}{c}\text { Magnesium } \\
\mu \mathrm{g} / \mathrm{g}\end{array}$ & $\begin{array}{c}\text { Strontium } \\
\mu \mathrm{g} / \mathrm{g}\end{array}$ & $\begin{array}{c}\text { Sodium } \\
\mu \mathrm{g} / \mathrm{g}\end{array}$ \\
\hline B1NDW3B & C5590 & 96.3 & $9.51 \mathrm{E}+00$ & $3.17 \mathrm{E}+00$ & $1.99 \mathrm{E}+00$ & $4.52 \mathrm{E}-02$ & $1.33 \mathrm{E}+01$ \\
\hline B1NDW3A & C5590 & 96.8 & $1.13 \mathrm{E}+01$ & $3.49 \mathrm{E}+00$ & $2.35 \mathrm{E}+00$ & $5.17 \mathrm{E}-02$ & $1.61 \mathrm{E}+01$ \\
\hline B1NDW3A DUP & C5590 & 96.8 & $1.18 \mathrm{E}+01$ & $3.56 \mathrm{E}+00$ & $2.46 \mathrm{E}+00$ & $5.41 \mathrm{E}-02$ & $1.65 \mathrm{E}+01$ \\
\hline B1NHV0B & C5592 & 62.3 & $5.26 \mathrm{E}+00$ & $4.00 \mathrm{E}+00$ & $1.91 \mathrm{E}+00$ & $3.49 \mathrm{E}-02$ & $2.45 \mathrm{E}+01$ \\
\hline B1NHV0A & C5592 & 62.8 & $4.93 \mathrm{E}+00$ & $3.89 \mathrm{E}+00$ & $1.77 \mathrm{E}+00$ & $3.46 \mathrm{E}-02$ & $1.98 \mathrm{E}+01$ \\
\hline B1NTC6B & C5598 & 50.3 & $6.33 \mathrm{E}+00$ & $3.83 \mathrm{E}+00$ & $1.55 \mathrm{E}+00$ & $3.91 \mathrm{E}-02$ & $2.04 \mathrm{E}+01$ \\
\hline B1NTC6A & C5598 & 50.8 & $9.00 \mathrm{E}+00$ & $4.20 \mathrm{E}+00$ & $2.21 \mathrm{E}+00$ & $5.44 \mathrm{E}-02$ & $1.94 \mathrm{E}+01$ \\
\hline B1NTC7B & C5598 & 59.8 & $8.64 \mathrm{E}+00$ & $4.78 \mathrm{E}+00$ & $2.87 \mathrm{E}+00$ & $5.33 \mathrm{E}-02$ & $1.60 \mathrm{E}+01$ \\
\hline B1NTC7A & C5598 & 60.3 & $7.43 \mathrm{E}+00$ & $4.87 \mathrm{E}+00$ & $2.41 \mathrm{E}+00$ & $4.48 \mathrm{E}-02$ & $1.54 \mathrm{E}+01$ \\
\hline B1NTC8B & C5598 & 82.3 & $1.58 \mathrm{E}+01$ & $4.95 \mathrm{E}+00$ & $4.89 \mathrm{E}+00$ & $8.25 \mathrm{E}-02$ & $1.81 \mathrm{E}+01$ \\
\hline B1NTC8B DUP & C5598 & 82.3 & $1.57 \mathrm{E}+01$ & $5.08 \mathrm{E}+00$ & $4.79 \mathrm{E}+00$ & $8.13 \mathrm{E}-02$ & $1.88 \mathrm{E}+01$ \\
\hline B1NTC8A & C5598 & 82.8 & $1.16 \mathrm{E}+01$ & $5.59 \mathrm{E}+00$ & $3.56 \mathrm{E}+00$ & $6.27 \mathrm{E}-02$ & $1.79 \mathrm{E}+01$ \\
\hline B1NTC9B & C5596 & 50.8 & $4.31 \mathrm{E}+00$ & $3.46 \mathrm{E}+00$ & $1.31 \mathrm{E}+00$ & $2.95 \mathrm{E}-02$ & $2.14 \mathrm{E}+01$ \\
\hline B1NTC9A & C5596 & 51.3 & $4.36 \mathrm{E}+00$ & $3.50 \mathrm{E}+00$ & $1.35 \mathrm{E}+00$ & $3.10 \mathrm{E}-02$ & $1.93 \mathrm{E}+01$ \\
\hline B1NTD0B & C5596 & 61.3 & $9.02 \mathrm{E}+00$ & $5.43 \mathrm{E}+00$ & $3.26 \mathrm{E}+00$ & $5.95 \mathrm{E}-02$ & $1.39 \mathrm{E}+01$ \\
\hline B1NTD0A & C5596 & 61.8 & $8.13 \mathrm{E}+00$ & $6.06 \mathrm{E}+00$ & $2.91 \mathrm{E}+00$ & $5.30 \mathrm{E}-02$ & $1.52 \mathrm{E}+01$ \\
\hline B1NTD1B & C5596 & 77.8 & $1.27 \mathrm{E}+01$ & $5.25 \mathrm{E}+00$ & $3.16 \mathrm{E}+00$ & $6.15 \mathrm{E}-02$ & $1.65 \mathrm{E}+01$ \\
\hline B1NTD1B DUP & C5596 & 77.8 & $1.30 \mathrm{E}+01$ & $5.05 \mathrm{E}+00$ & $3.30 \mathrm{E}+00$ & $6.40 \mathrm{E}-02$ & $1.68 \mathrm{E}+01$ \\
\hline
\end{tabular}




\begin{tabular}{|c|c|c|c|c|c|c|c|}
\hline B1NTD1A & ב5596 & 78.3 & $\begin{array}{l}1.33 \mathrm{E}+01 \\
\end{array}$ & $\overline{5.40 \mathrm{E}+00}$ & $3.38 \mathrm{E}+00$ & 6.71E-02 & $1.75 \mathrm{E}+01$ \\
\hline B1NTD2B & C5596 & 82.8 & $8.70 \mathrm{E}+00$ & $5.54 \mathrm{E}+00$ & $3.01 \mathrm{E}+00$ & $4.87 \mathrm{E}-02$ & $1.49 \mathrm{E}+01$ \\
\hline B1NTD2A & C5596 & 83.3 & $8.24 \mathrm{E}+00$ & $4.74 \mathrm{E}+00$ & $2.73 \mathrm{E}+00$ & $4.66 \mathrm{E}-02$ & $1.41 \mathrm{E}+01$ \\
\hline B1NTD3B & C5600 & 50.3 & $9.00 \mathrm{E}+00$ & $3.62 \mathrm{E}+00$ & $2.17 \mathrm{E}+00$ & $5.61 \mathrm{E}-02$ & $1.47 \mathrm{E}+01$ \\
\hline B1NTD3A & C5600 & 50.8 & $8.51 \mathrm{E}+00$ & $3.82 \mathrm{E}+00$ & $1.86 \mathrm{E}+00$ & $5.08 \mathrm{E}-02$ & $1.61 \mathrm{E}+01$ \\
\hline B1NTD4B & C5600 & 60.3 & $7.33 \mathrm{E}+00$ & $5.56 \mathrm{E}+00$ & $2.39 \mathrm{E}+00$ & $4.15 \mathrm{E}-02$ & $1.32 \mathrm{E}+01$ \\
\hline B1NTD4A & C5600 & 60.8 & $7.98 \mathrm{E}+00$ & $5.08 \mathrm{E}+00$ & $2.52 \mathrm{E}+00$ & $4.97 \mathrm{E}-02$ & $1.29 \mathrm{E}+01$ \\
\hline B1NTD5B & C5600 & 82.3 & $1.74 \mathrm{E}+01$ & $7.43 \mathrm{E}+00$ & $5.31 \mathrm{E}+00$ & $8.96 \mathrm{E}-02$ & $2.52 \mathrm{E}+01$ \\
\hline B1NTD5A & C5600 & 82.8 & $9.46 \mathrm{E}+00$ & $5.94 \mathrm{E}+00$ & $2.68 \mathrm{E}+00$ & $4.85 \mathrm{E}-02$ & $1.82 \mathrm{E}+01$ \\
\hline B1P1K6B & C5600 & 88.8 & $9.57 \mathrm{E}+00$ & $3.99 \mathrm{E}+00$ & $2.33 \mathrm{E}+00$ & $4.86 \mathrm{E}-02$ & $1.46 \mathrm{E}+01$ \\
\hline B1P1K6A & C5600 & 89.3 & $1.33 \mathrm{E}+01$ & $4.27 \mathrm{E}+00$ & $3.17 \mathrm{E}+00$ & $6.52 \mathrm{E}-02$ & $1.72 \mathrm{E}+01$ \\
\hline B1P3F9B & C5604 & 50.8 & $6.10 \mathrm{E}+00$ & $3.74 \mathrm{E}+00$ & $1.80 \mathrm{E}+00$ & $4.40 \mathrm{E}-02$ & $2.52 \mathrm{E}+01$ \\
\hline B1P3F9A & C5604 & 51.3 & $4.02 \mathrm{E}+00$ & $3.16 \mathrm{E}+00$ & $1.30 \mathrm{E}+00$ & $3.24 \mathrm{E}-02$ & $1.91 \mathrm{E}+01$ \\
\hline B1P3H0B & C5602 & 51.8 & $5.44 \mathrm{E}+00$ & $3.66 \mathrm{E}+00$ & $2.01 \mathrm{E}+00$ & $3.68 \mathrm{E}-02$ & $1.66 \mathrm{E}+01$ \\
\hline B1P3H0A & C5602 & 52.3 & $4.35 \mathrm{E}+00$ & $2.91 \mathrm{E}+00$ & $1.82 \mathrm{E}+00$ & $3.33 \mathrm{E}-02$ & $1.31 \mathrm{E}+01$ \\
\hline B1P3H1B & C5602 & 67.8 & $6.36 \mathrm{E}+00$ & $3.77 \mathrm{E}+00$ & $2.17 \mathrm{E}+00$ & $3.81 \mathrm{E}-02$ & $1.08 \mathrm{E}+01$ \\
\hline B1P3H1A & C5602 & 68.3 & $9.22 \mathrm{E}+00$ & $3.87 \mathrm{E}+00$ & $2.83 \mathrm{E}+00$ & $5.45 \mathrm{E}-02$ & $1.34 \mathrm{E}+01$ \\
\hline B1P3H2B & C5602 & 82.8 & $1.09 \mathrm{E}+01$ & $5.57 \mathrm{E}+00$ & $3.04 \mathrm{E}+00$ & $5.77 \mathrm{E}-02$ & $1.86 \mathrm{E}+01$ \\
\hline B1P3H2A & C5602 & 83.3 & $7.08 \mathrm{E}+00$ & $4.03 \mathrm{E}+00$ & $1.97 \mathrm{E}+00$ & $3.69 \mathrm{E}-02$ & $1.09 \mathrm{E}+01$ \\
\hline B1PBB0B & C5602 & 91.8 & $1.32 \mathrm{E}+01$ & $4.26 \mathrm{E}+00$ & $2.77 \mathrm{E}+00$ & $6.49 \mathrm{E}-02$ & $1.57 \mathrm{E}+01$ \\
\hline B1PBB0A & C5602 & 92.3 & $9.73 \mathrm{E}+00$ & $3.88 \mathrm{E}+00$ & $2.07 \mathrm{E}+00$ & $4.76 \mathrm{E}-02$ & $1.34 \mathrm{E}+01$ \\
\hline B1PBB1B & C5606 & 51.8 & $1.38 \mathrm{E}+01$ & $4.27 \mathrm{E}+00$ & $3.09 \mathrm{E}+00$ & $8.46 \mathrm{E}-02$ & $1.86 \mathrm{E}+01$ \\
\hline B1PBB1A & C5606 & 52.3 & $1.53 \mathrm{E}+01$ & $5.11 \mathrm{E}+00$ & $3.59 \mathrm{E}+00$ & $9.69 \mathrm{E}-02$ & $2.06 \mathrm{E}+01$ \\
\hline B1PBB1A DUP & C5606 & 52.3 & $1.44 \mathrm{E}+01$ & $5.24 \mathrm{E}+00$ & $3.47 \mathrm{E}+00$ & $9.31 \mathrm{E}-02$ & $2.03 \mathrm{E}+01$ \\
\hline B1PK51B & C5608 & 64.3 & $5.76 E-01$ & $(1.70 E+00)$ & $(1.05 E-01)$ & $(6.32 E-03)$ & $1.91 E+02$ \\
\hline B1PK51A & C5608 & 64.8 & $4.80 E-01$ & $(1.64 E+00)$ & $(7.75 E-02)$ & $(3.82 E-03)$ & $1.43 E+02$ \\
\hline B1PK52B & C5608 & 85.8 & $7.88 E-01$ & $(3.20 E+00)$ & $(1.05 E-01)$ & (6.06E-03) & $6.89 \mathrm{E}+02$ \\
\hline B1PK52A & C5608 & 86.3 & $6.35 E-01$ & $(2.36 E+00)$ & $(2.72 E-02)$ & $(5.22 E-03)$ & $6.36 \mathrm{E}+02$ \\
\hline B1PK53B & C5608 & 97.8 & $6.84 E-01$ & $(2.65 E+00)$ & $(1.40 E-01)$ & $(7.40 E-03)$ & $4.18 \mathrm{E}+02$ \\
\hline B1PK53A & C5608 & 98.3 & $5.59 E-01$ & $(1.63 E+00)$ & $(7.78 E-02)$ & (7.32E-03) & $2.39 \mathrm{E}+02$ \\
\hline
\end{tabular}

The water-extractable aluminum, iron, silicon, and sulfur in the U Farm direct push sediments are shown in Table 4.23. The sulfur data were converted to water-extractable sulfur as sulfate so that the results could be compared to the IC data presented in Table 4.21. Water-soluble iron was elevated in samples from probe hole C5608. It appears that these elevated concentrations of water-extractable iron are a result of some chemical reactions (dissolution/precipitation) between alkaline tank fluids and native sediments that formed precipitates of amorphous iron phases that are more water soluble than their crystalline counterparts in the native sediments. Additionally, the second string of samples collected from push hole C5608 contained elevated water-extractable silicon. These results further support the hypothesis that the vadose zone sediments in the vicinity of this probe hole have been chemically altered due to interaction with tank-related waste. The agreement between sulfate directly measured in the water extracts using ion chromatography and indirectly measured by converting the ICP measurements for sulfur to sulfate was very good, except for those samples retrieved from push hole C5608 where differences of as much as $50 \%$ were calculated between the two data sets, indicating that some of the sulfur measured in the water extracts via ICP-OES could be present in some form other than sulfate. 
Table 4.23. Water-Extractable Cations in the U Tank Farm Core Samples ( $\mu \mathrm{g} / \mathrm{g}$ dry sediment)

\begin{tabular}{|c|c|c|c|c|c|c|}
\hline $\begin{array}{c}\text { Sample } \\
\text { ID }\end{array}$ & $\begin{array}{c}\text { Probe Hole } \\
\text { ID } \\
\end{array}$ & $\begin{array}{l}\text { Mid-Depth } \\
\text { ft bgs }\end{array}$ & $\begin{array}{c}\text { Aluminum } \\
\mu \mathrm{g} / \mathrm{g}\end{array}$ & $\begin{array}{l}\text { Iron } \\
\mu \mathrm{g} / \mathrm{g}\end{array}$ & $\begin{array}{c}\text { Sulfur as } \mathrm{SO}_{4}{ }^{2-} \\
\mu \mathrm{g} / \mathrm{g}\end{array}$ & $\begin{array}{c}\begin{array}{c}\text { Silicon } \\
\mu \mathrm{g} / \mathrm{g}\end{array} \\
\end{array}$ \\
\hline B1NDW3B & C5590 & 96.3 & $4.85 \mathrm{E}-02$ & $6.70 \mathrm{E}-02$ & $1.00 \mathrm{E}+01$ & $8.96 \mathrm{E}+00$ \\
\hline B1NDW3A & C5590 & 96.8 & $4.20 \mathrm{E}-02$ & $6.42 \mathrm{E}-02$ & $1.21 \mathrm{E}+01$ & $8.92 \mathrm{E}+00$ \\
\hline B1NDW3A DUP & C5590 & 96.8 & $5.51 \mathrm{E}-02$ & $5.57 \mathrm{E}-02$ & $1.30 \mathrm{E}+01$ & $8.86 \mathrm{E}+00$ \\
\hline B1NHV0B & C5592 & 62.3 & $3.24 \mathrm{E}-01$ & $4.87 \mathrm{E}-01$ & $1.41 \mathrm{E}+01$ & $1.12 \mathrm{E}+01$ \\
\hline B1NHV0A & C5592 & 62.8 & $3.40 \mathrm{E}-01$ & $4.77 \mathrm{E}-01$ & $1.19 \mathrm{E}+01$ & $1.01 \mathrm{E}+01$ \\
\hline B1NTC6B & C5598 & 50.3 & $9.92 \mathrm{E}-02$ & $1.46 \mathrm{E}-01$ & $(4.98 \mathrm{E}+00)$ & $9.65 \mathrm{E}+00$ \\
\hline B1NTC6A & C5598 & 50.8 & $7.31 \mathrm{E}-02$ & $1.25 \mathrm{E}-01$ & $1.05 \mathrm{E}+01$ & $9.40 \mathrm{E}+00$ \\
\hline B1NTC7B & C5598 & 59.8 & $6.47 \mathrm{E}-02$ & $4.42 \mathrm{E}-02$ & $1.50 \mathrm{E}+01$ & $8.35 \mathrm{E}+00$ \\
\hline B1NTC7A & C5598 & 60.3 & $7.68 \mathrm{E}-02$ & $7.51 \mathrm{E}-02$ & $1.31 \mathrm{E}+01$ & $7.31 \mathrm{E}+00$ \\
\hline B1NTC8B & C5598 & 82.3 & $(2.28 \mathrm{E}-02)$ & $(1.38 \mathrm{E}-02)$ & $2.43 \mathrm{E}+01$ & $8.45 \mathrm{E}+00$ \\
\hline B1NTC8B DUP & C5598 & 82.3 & (2.59E-02) & $1.68 \mathrm{E}-02$ & $2.49 \mathrm{E}+01$ & $8.56 \mathrm{E}+00$ \\
\hline B1NTC8A & C5598 & 82.8 & $3.40 \mathrm{E}-02$ & $2.29 \mathrm{E}-02$ & $2.11 \mathrm{E}+01$ & $7.82 \mathrm{E}+00$ \\
\hline B1NTC9B & C5596 & 50.8 & $9.60 \mathrm{E}-02$ & $9.09 \mathrm{E}-02$ & $(3.50 \mathrm{E}+00)$ & $1.04 \mathrm{E}+01$ \\
\hline B1NTC9A & C5596 & 51.3 & $1.53 \mathrm{E}-01$ & $1.63 \mathrm{E}-01$ & $(2.47 \mathrm{E}+00)$ & $1.01 \mathrm{E}+01$ \\
\hline B1NTD0B & C5596 & 61.3 & $5.86 \mathrm{E}-02$ & 4.73E-02 & $1.65 \mathrm{E}+01$ & $8.01 \mathrm{E}+00$ \\
\hline B1NTD0A & C5596 & 61.8 & $6.99 \mathrm{E}-02$ & 4.69E-02 & $1.64 \mathrm{E}+01$ & $7.28 \mathrm{E}+00$ \\
\hline B1NTD1B & C5596 & 77.8 & $5.57 \mathrm{E}-02$ & $4.47 \mathrm{E}-02$ & $2.18 \mathrm{E}+01$ & $8.37 \mathrm{E}+00$ \\
\hline B1NTD1B DUP & C5596 & 77.8 & $4.58 \mathrm{E}-02$ & $3.85 \mathrm{E}-02$ & $2.43 \mathrm{E}+01$ & $7.72 \mathrm{E}+00$ \\
\hline B1NTD1A & C5596 & 78.3 & $4.56 \mathrm{E}-02$ & $3.95 \mathrm{E}-02$ & $2.79 \mathrm{E}+01$ & $8.06 \mathrm{E}+00$ \\
\hline B1NTD2B & C5596 & 82.8 & $7.21 \mathrm{E}-02$ & $4.45 \mathrm{E}-02$ & $1.09 \mathrm{E}+01$ & $7.12 \mathrm{E}+00$ \\
\hline B1NTD2A & C5596 & 83.3 & $7.52 \mathrm{E}-02$ & $4.41 \mathrm{E}-02$ & $9.82 \mathrm{E}+00$ & $7.69 \mathrm{E}+00$ \\
\hline B1NTD3B & C5600 & 50.3 & $4.69 \mathrm{E}-02$ & $5.77 \mathrm{E}-02$ & $(8.15 \mathrm{E}+00)$ & $9.68 \mathrm{E}+00$ \\
\hline B1NTD3A & C5600 & 50.8 & $7.73 \mathrm{E}-02$ & 1.19E-01 & $(6.62 \mathrm{E}+00)$ & $8.22 \mathrm{E}+00$ \\
\hline B1NTD4B & C5600 & 60.3 & $3.15 \mathrm{E}-01$ & $3.81 \mathrm{E}-01$ & $(7.31 \mathrm{E}+00)$ & $9.21 \mathrm{E}+00$ \\
\hline B1NTD4A & C5600 & 60.8 & $3.52 \mathrm{E}-01$ & $4.15 \mathrm{E}-01$ & $9.64 \mathrm{E}+00$ & $1.01 \mathrm{E}+01$ \\
\hline B1NTD5B & C5600 & 82.3 & $1.81 \mathrm{E}-01$ & $2.21 \mathrm{E}-01$ & $4.55 \mathrm{E}+01$ & $9.46 \mathrm{E}+00$ \\
\hline B1NTD5A & C5600 & 82.8 & $2.91 \mathrm{E}-01$ & 3.32E-01 & $2.15 \mathrm{E}+01$ & $8.44 \mathrm{E}+00$ \\
\hline B1P1K6B & C5600 & 88.8 & $8.13 \mathrm{E}-02$ & $6.53 \mathrm{E}-02$ & $1.26 \mathrm{E}+01$ & $7.13 \mathrm{E}+00$ \\
\hline B1P1K6A & C5600 & 89.3 & $4.59 \mathrm{E}-02$ & $3.78 \mathrm{E}-02$ & $1.95 \mathrm{E}+01$ & $7.79 \mathrm{E}+00$ \\
\hline B1P3F9B & C5604 & 50.8 & $9.14 \mathrm{E}-02$ & $1.89 \mathrm{E}-01$ & $(6.47 \mathrm{E}+00)$ & $9.51 \mathrm{E}+00$ \\
\hline B1P3F9A & C5604 & 51.3 & $1.82 \mathrm{E}-01$ & $2.58 \mathrm{E}-01$ & $(6.62 \mathrm{E}+00)$ & $9.19 \mathrm{E}+00$ \\
\hline B1P3H0B & C5602 & 51.8 & $1.69 \mathrm{E}-01$ & $2.15 \mathrm{E}-01$ & $(5.06 \mathrm{E}+00)$ & $7.96 \mathrm{E}+00$ \\
\hline B1P3H0A & C5602 & 52.3 & $2.06 \mathrm{E}-01$ & $1.72 \mathrm{E}-01$ & $(2.10 \mathrm{E}+00)$ & $6.38 \mathrm{E}+00$ \\
\hline B1P3H1B & C5602 & 67.8 & $1.48 \mathrm{E}-01$ & $1.28 \mathrm{E}-01$ & $(7.37 \mathrm{E}+00)$ & $7.21 \mathrm{E}+00$ \\
\hline B1P3H1A & C5602 & 68.3 & $9.15 \mathrm{E}-02$ & $1.14 \mathrm{E}-01$ & $1.35 \mathrm{E}+01$ & $7.96 \mathrm{E}+00$ \\
\hline $\mathrm{B} 1 \mathrm{P} 3 \mathrm{H} 2 \mathrm{~B}$ & C5602 & 82.8 & $5.78 \mathrm{E}-02$ & 4.46E-02 & $2.44 \mathrm{E}+01$ & $7.51 \mathrm{E}+00$ \\
\hline B1P3H2A & C5602 & 83.3 & $1.01 \mathrm{E}-01$ & $8.53 \mathrm{E}-02$ & $1.09 \mathrm{E}+01$ & $6.83 \mathrm{E}+00$ \\
\hline B1PBB0B & C5602 & 91.8 & $4.57 \mathrm{E}-02$ & $2.60 \mathrm{E}-02$ & $2.75 \mathrm{E}+01$ & $6.67 \mathrm{E}+00$ \\
\hline B1PBB0A & C5602 & 92.3 & $6.45 \mathrm{E}-02$ & $5.18 \mathrm{E}-02$ & $2.02 \mathrm{E}+01$ & $7.51 \mathrm{E}+00$ \\
\hline B1PBB1B & C5606 & 51.8 & $8.09 \mathrm{E}-02$ & $1.23 \mathrm{E}-01$ & $3.24 \mathrm{E}+01$ & $9.68 \mathrm{E}+00$ \\
\hline B1PBB1A & C5606 & 52.3 & $(5.76 \mathrm{E}-02)$ & $7.74 \mathrm{E}-02$ & $3.25 \mathrm{E}+01$ & $9.09 \mathrm{E}+00$ \\
\hline B1PBB1A DUP & C5606 & 52.3 & (7.04E-02) & $1.02 \mathrm{E}-01$ & $3.13 \mathrm{E}+01$ & $9.13 \mathrm{E}+00$ \\
\hline B1PK51B & C5608 & 64.3 & $3.49 \mathrm{E}-01$ & $1.11 \mathrm{E}+00$ & $1.94 \mathrm{E}+01$ & $9.04 \mathrm{E}+00$ \\
\hline B1PK51A & C5608 & 64.8 & $4.05 \mathrm{E}-01$ & $2.16 \mathrm{E}-01$ & $1.03 \mathrm{E}+01$ & $8.32 \mathrm{E}+00$ \\
\hline
\end{tabular}




\begin{tabular}{||l|c|c|c|c|c|c||}
\hline B1PK52B & C5608 & 85.8 & $3.66 \mathrm{E}-01$ & $\mathbf{7 . 2 9 E}-\mathbf{0 1}$ & $4.21 \mathrm{E}+01$ & $\mathbf{8 . 2 8 E}+\mathbf{0 1}$ \\
\hline B1PK52A & C5608 & 86.3 & $1.57 \mathrm{E}-01$ & $2.96 \mathrm{E}-01$ & $3.80 \mathrm{E}+01$ & $\mathbf{6 . 7 2 E}+\mathbf{0 1}$ \\
\hline B1PK53B & C5608 & 97.8 & $1.70 \mathrm{E}-01$ & $3.75 \mathrm{E}-01$ & $5.06 \mathrm{E}+01$ & $7.15 \mathrm{E}+00$ \\
\hline B1PK53A & C5608 & 98.3 & $3.07 \mathrm{E}-01$ & $1.22 \mathrm{E}-01$ & $2.35 \mathrm{E}+01$ & $7.85 \mathrm{E}+00$ \\
\hline $\begin{array}{l}\text { Bold values denote elevated concentrations. } \\
\text { Parentheses indicate reported value was less than the limit of quantification for the analysis. }\end{array}$ \\
\hline
\end{tabular}

The water extract data for potentially mobile metals, such as technetium-99, uranium-238, chromium, and molybdenum are shown in Table 4.24. As seen in Table 4.24, samples collected from two of the push holes contained quantifiable activities of technetium-99; however, push hole C5602 only contained a trace amount (at less than $0.2 \mathrm{pCi} / \mathrm{g}$ ). Conversely, all of the samples analyzed from push hole C5608, which was emplaced adjacent to tank 241-U-110, contained appreciable activities of technetium-99. The technetium-99 measured in push hole $\mathrm{C} 5608$ ranged from 0.054 to $48.8 \mathrm{pCi} / \mathrm{g}$. The two samples containing the lowest technetium-99 activities, at 0.163 and $0.054 \mathrm{pCi} / \mathrm{g}$, were collected shallowest (approximately $65 \mathrm{ft}$ bgs) in the push hole. The highest activity samples (48.8 and $41.9 \mathrm{pCi} / \mathrm{g})$ were collected from the middle sampling depth in the push hole, at approximately $86 \mathrm{ft}$ bgs. The two deepest samples analyzed from push hole C5608 contained approximately 20\%-25\% as much technetium-99 as the samples from $86 \mathrm{ft}$ bgs. Although the total vertical extent of contamination remains unknown at this location, a rudimentary profile can be fashioned with the depth-discrete data acquired thus far (Figure 4.3). Based on these data, in conjunction with the cation results presented in Table 4.24, it is possible that the leading edge of the waste plume is near the maximum depth sampled. Based on the major cation data, in conjunction with the technetium-99 data, the concentration profiles are decreasing with depth beginning with the samples collected from $86 \mathrm{ft}$ bgs. However, it is not possible to confirm this supposition without additional depth-discrete samples.

Elevated water-leachable chromium (up to $6.0 \mu \mathrm{g} / \mathrm{g}$ ) was observed in the samples retrieved from push hole C5608. For comparative purposes, the background borehole contained $0.06 \mu \mathrm{g} / \mathrm{g}$ or less waterextractable chromium. The tank 241-U-110 leak event is estimated to have released $25.4 \mathrm{~kg}$ of chromium to the vadose zone (Wood and Jones 2003). None of the other direct push samples analyzed contained appreciable quantities of water-extractable chromium. Additionally, none of the U Farm direct push samples analyzed (including those from push hole C5608) contained elevated concentrations of molybdenum, silver, or lead.

Significantly elevated water-leachable uranium-238 was only found in samples from push hole C5602, which was emplaced just southeast of tank 241-U-105. While the background borehole sediments had water-extractable uranium concentrations ranging from $1.93 \mathrm{E}-04$ to $1.79 \mathrm{E}-03 \mu \mathrm{g} / \mathrm{g}$, sediments retrieved from push hole C5602 contained as much as $5.16 \mu \mathrm{g} / \mathrm{g}$ water-extractable uranium (Table 4.24 and Figure 4.4). It does not appear that all of the samples retrieved from push hole C5602 contained contaminant uranium. Water extract data for the two deepest samples analyzed appeared to be representative of natural uranium. Sediments retrieved from push holes C5606 and C5608, emplaced near tank 241-U-112 and 241-U-110, respectively, could contain small amounts of contaminant uranium (based on the water-extract data), but at $0.13 \mu \mathrm{g} / \mathrm{g}$ or less uranium, the samples are not significantly elevated above background concentrations. These results are not surprising given that the 241-U-112 and 241-U-110 tank leaks were estimated to have released less than $0.01 \mathrm{Ci}$ (each) of uranium-238 to the vadose zone (Wood and Jones 2003). 
Table 4.24. Water-Extractable Mobile Metals in the U Tank Farm Core Samples ( $\mu \mathrm{g} / \mathrm{g}$ dry sediment)

\begin{tabular}{|c|c|c|c|c|c|c|c|c|}
\hline $\begin{array}{l}\text { Sample } \\
\text { ID }\end{array}$ & $\begin{array}{l}\text { Probe Hole } \\
\text { ID }\end{array}$ & $\begin{array}{l}\text { Mid-Depth } \\
\mathrm{ft} \text { bgs }\end{array}$ & $\begin{array}{c}\text { Technetium-99 } \\
\text { pCi/g }\end{array}$ & $\begin{array}{c}\text { Uranium-238 } \\
\mu \mathrm{g} / \mathrm{g}\end{array}$ & $\begin{array}{c}\text { Chromium } \\
\mu \mathrm{g} / \mathrm{g}\end{array}$ & $\begin{array}{l}\text { Molybdenum } \\
\mu \mathrm{g} / \mathrm{g}\end{array}$ & $\begin{array}{l}\text { Silver } \\
\mu \mathrm{g} / \mathrm{g}\end{array}$ & $\begin{array}{l}\text { Lead } \\
\mu \mathrm{g} / \mathrm{g}\end{array}$ \\
\hline "B1NDW3B & בC5590 & 96.3 & (1.02E-02) & "3.91E-04 & (2.04E-03) & (4.57E-02 & (5.10E-05) & $\begin{array}{l}(1.34 \mathrm{E}-04) \\
\end{array}$ \\
\hline B1NDW3A & C5590 & 96.8 & $\begin{array}{l}(1.70 \mathrm{E}-02) \\
\end{array}$ & $4.45 \mathrm{E}-04$ & $2.64 \mathrm{E}-03$ & $7.80 \mathrm{E}-02$ & $\begin{array}{l}(2.80 \mathrm{E}-05) \\
\end{array}$ & $\begin{array}{l}(1.94 \mathrm{E}-04) \\
\end{array}$ \\
\hline B1NDW3A DUP & C5590 & 96.8 & $<1.70 \mathrm{E}-01$ & 4.69E-04 & $2.78 \mathrm{E}-03$ & $7.50 \mathrm{E}-02$ & $(2.50 \mathrm{E}-05)$ & $(1.11 \mathrm{E}-04)$ \\
\hline B1NHV0B & C5592 & 62.3 & $<1.70 \mathrm{E}-01$ & $1.18 \mathrm{E}-03$ & (1.61E-03) & $3.34 \mathrm{E}-02$ & $(1.75 \mathrm{E}-05)$ & $(6.27 \mathrm{E}-04)$ \\
\hline B1NHV0A & C5592 & 62.8 & $<1.69 \mathrm{E}-01$ & $8.58 \mathrm{E}-04$ & (1.21E-03) & $3.06 \mathrm{E}-02$ & $(2.15 \mathrm{E}-05)$ & $1.43 \mathrm{E}-03$ \\
\hline B1NTC6B & C5598 & 50.3 & $<1.71 \mathrm{E}-01$ & $1.20 \mathrm{E}-03$ & $(1.51 \mathrm{E}-03)$ & $6.06 \mathrm{E}-02$ & $(3.52 \mathrm{E}-06)$ & $(2.57 \mathrm{E}-04)$ \\
\hline B1NTC6A & C5598 & 50.8 & $<1.70 \mathrm{E}-01$ & $8.32 \mathrm{E}-04$ & (6.42E-04) & $4.51 \mathrm{E}-02$ & $(7.50 \mathrm{E}-06)$ & $(2.56 \mathrm{E}-04)$ \\
\hline B1NTC7B & C5598 & 59.8 & $<1.70 \mathrm{E}-01$ & $4.66 \mathrm{E}-04$ & $(1.21 \mathrm{E}-03)$ & $5.22 \mathrm{E}-02$ & (6.00E-06) & $(2.39 \mathrm{E}-04)$ \\
\hline B1NTC7A & C5598 & 60.3 & $<1.70 \mathrm{E}-01$ & $3.05 \mathrm{E}-04$ & (6.78E-04) & $5.58 \mathrm{E}-02$ & (6.00E-06) & $(2.00 \mathrm{E}-04)$ \\
\hline B1NTC8B & C5598 & 82.3 & $<1.70 \mathrm{E}-01$ & $7.55 \mathrm{E}-04$ & $(2.28 \mathrm{E}-03)$ & $4.07 \mathrm{E}-02$ & $(8.50 \mathrm{E}-06)$ & $(4.50 \mathrm{E}-05)$ \\
\hline B1NTC8B DUP & C5598 & 82.3 & $<1.70 \mathrm{E}-01$ & 7.24E-04 & (2.36E-03) & $4.13 \mathrm{E}-02$ & (3.01E-06) & $(8.93 \mathrm{E}-05)$ \\
\hline$\overline{B 1 N T C 8 A}$ & C5598 & 82.8 & $(1.02 \mathrm{E}-02)$ & $4.81 \mathrm{E}-04$ & $\begin{array}{l}(1.97 \mathrm{E}-03) \\
\end{array}$ & $7.22 \mathrm{E}-02$ & $(4.00 \mathrm{E}-06)$ & $\begin{array}{c}(4.10 \mathrm{E}-05) \\
\end{array}$ \\
\hline B1NTC9B & C5596 & 50.8 & $<1.70 \mathrm{E}-01$ & $1.30 \mathrm{E}-03$ & (6.18E-04) & $2.47 \mathrm{E}-02$ & (6.01E-06) & $(1.19 \mathrm{E}-04)$ \\
\hline B1NTC9A & C5596 & 51.3 & $<1.73 \mathrm{E}-01$ & $9.78 \mathrm{E}-04$ & (6.57E-04) & $2.55 \mathrm{E}-02$ & (4.09E-06) & $(1.96 \mathrm{E}-04)$ \\
\hline B1NTD0B & C5596 & 61.3 & $<1.70 \mathrm{E}-01$ & 5.90E-04 & $(1.59 \mathrm{E}-03)$ & $7.95 \mathrm{E}-02$ & $(1.60 \mathrm{E}-05)$ & $(1.15 \mathrm{E}-04)$ \\
\hline B1NTD0A & C5596 & 61.8 & $<1.73 \mathrm{E}-01$ & $3.70 \mathrm{E}-04$ & (1.14E-03) & $1.62 \mathrm{E}-01$ & (8.67E-06) & $(9.02 \mathrm{E}-05)$ \\
\hline B1NTD1B & C5596 & 77.8 & $(6.05 \mathrm{E}-02)$ & $4.31 \mathrm{E}-04$ & $(2.33 \mathrm{E}-03)$ & $6.33 \mathrm{E}-02$ & $(5.01 \mathrm{E}-06)$ & $(5.01 \mathrm{E}-05)$ \\
\hline B1NTD1B DUP & C5596 & 77.8 & $(1.66 \mathrm{E}-01)$ & $4.47 \mathrm{E}-04$ & $(2.38 \mathrm{E}-03)$ & $6.50 \mathrm{E}-02$ & $(1.20 \mathrm{E}-05)$ & $(4.05 \mathrm{E}-05)$ \\
\hline B1NTD1A & C5596 & 78.3 & $(7.80 \mathrm{E}-02)$ & $4.42 \mathrm{E}-04$ & $3.49 \mathrm{E}-03$ & $7.68 \mathrm{E}-02$ & (7.00E-06) & (3.18E-04) \\
\hline B1NTD2B & C5596 & 82.8 & $(9.16 \mathrm{E}-02)$ & $2.22 \mathrm{E}-04$ & $(1.47 \mathrm{E}-03)$ & $3.71 \mathrm{E}-02$ & $(1.00 \mathrm{E}-06)$ & $(4.65 \mathrm{E}-05)$ \\
\hline B1NTD2A & C5596 & 83.3 & (1.19E-01) & 1.89E-04 & (1.04E-03) & $1.91 \mathrm{E}-02$ & (4.00E-06) & $(4.60 \mathrm{E}-05)$ \\
\hline B1NTD3B & C5600 & 50.3 & $<1.70 \mathrm{E}-01$ & $1.02 \mathrm{E}-03$ & (6.06E-04) & $3.12 \mathrm{E}-02$ & $(6.50 \mathrm{E}-06)$ & $(1.61 \mathrm{E}-04)$ \\
\hline B1NTD3A & C5600 & 50.8 & $(1.02 \mathrm{E}-02)$ & 7.92E-04 & (5.13E-04) & $1.08 \mathrm{E}-01$ & $(6.50 \mathrm{E}-06)$ & $(2.66 \mathrm{E}-04)$ \\
\hline B1NTD4B & C5600 & 60.3 & $<1.70 \mathrm{E}-01$ & $3.73 \mathrm{E}-04$ & $2.73 \mathrm{E}-03$ & $7.66 \mathrm{E}-02$ & (3.50E-06) & $(3.19 \mathrm{E}-04)$ \\
\hline B1NTD4A & C5600 & 60.8 & $<1.70 \mathrm{E}-01$ & $3.65 \mathrm{E}-04$ & $2.66 \mathrm{E}-03$ & $4.87 \mathrm{E}-02$ & $(9.50 \mathrm{E}-06)$ & $(4.66 \mathrm{E}-04)$ \\
\hline B1NTD5B & C5600 & 82.3 & $(1.71 \mathrm{E}-02)$ & 8.99E-04 & $3.15 \mathrm{E}-03$ & $1.58 \mathrm{E}-01$ & (7.06E-06) & $(2.88 \mathrm{E}-04)$ \\
\hline B1NTD5A & C5600 & 82.8 & $(1.02 \mathrm{E}-02)$ & $3.05 \mathrm{E}-04$ & $2.59 \mathrm{E}-03$ & $1.30 \mathrm{E}-01$ & $(4.53 \mathrm{E}-06)$ & $(2.97 \mathrm{E}-04)$ \\
\hline B1P1K6B & C5600 & 88.8 & (6.10E-02) & $2.06 \mathrm{E}-04$ & $(1.98 \mathrm{E}-03)$ & $4.28 \mathrm{E}-02$ & $(1.50 \mathrm{E}-06)$ & $(6.25 \mathrm{E}-05)$ \\
\hline B1P1K6A & C5600 & 89.3 & $(3.73 \mathrm{E}-02)$ & $4.34 \mathrm{E}-04$ & (2.46E-03) & $5.16 \mathrm{E}-02$ & $(1.50 \mathrm{E}-06)$ & (7.05E-05) \\
\hline B1P3F9B & C5604 & 50.8 & $(3.73 \mathrm{E}-02)$ & $2.30 \mathrm{E}-03$ & $(1.50 \mathrm{E}-03)$ & $8.67 \mathrm{E}-02$ & $(5.00 \mathrm{E}-06)$ & $(4.12 \mathrm{E}-04)$ \\
\hline
\end{tabular}




\begin{tabular}{|c|c|c|c|c|c|c|c|c|}
\hline B1P3F9A & בC5604 & "51.3 & (3.05E-02) & "1.50E-03 & (8.04E-04) & "6.74E-02 & (6.50E-06) & (3.35E-04) \\
\hline B1P3H0B & C5602 & 51.8 & $<1.70 \mathrm{E}-01$ & $1.46 \mathrm{E}+00$ & $(1.38 \mathrm{E}-03)$ & $5.27 \mathrm{E}-02$ & $(4.00 \mathrm{E}-06)$ & (2.84E-04) \\
\hline B1P3H0A & C5602 & 52.3 & $<1.70 \mathrm{E}-01$ & $1.34 \mathrm{E}+00$ & $(1.03 \mathrm{E}-03)$ & $3.55 \mathrm{E}-02$ & $(5.00 \mathrm{E}-06)$ & (1.70E-04) \\
\hline B1P3H1B & C5602 & 67.8 & $(1.70 \mathrm{E}-02)$ & $1.72 \mathrm{E}+00$ & $(1.21 \mathrm{E}-03)$ & $2.50 \mathrm{E}-02$ & (3.50E-06) & (7.66E-05) \\
\hline B1P3H1A & $\mathrm{C} 5602$ & 68.3 & $(2.71 \mathrm{E}-02)$ & $5.16 \mathrm{E}+00$ & $(1.05 \mathrm{E}-03)$ & $4.86 \mathrm{E}-02$ & $(5.00 \mathrm{E}-06)$ & $(2.00 \mathrm{E}-04)$ \\
\hline B1P3H2B & $\mathrm{C} 5602$ & 82.8 & $(1.22 \mathrm{E}-01)$ & $2.52 \mathrm{E}+00$ & $(9.31 \mathrm{E}-04)$ & $6.62 \mathrm{E}-02$ & $(5.00 \mathrm{E}-06)$ & $(1.89 \mathrm{E}-04)$ \\
\hline B1P3H2A & C5602 & 83.3 & $(1.02 \mathrm{E}-01)$ & 8.35E-01 & (6.64E-04) & $3.50 \mathrm{E}-02$ & $(3.50 \mathrm{E}-06)$ & (8.50E-05) \\
\hline B1PBB0B & C5602 & 91.8 & 1.93E-01 & $3.57 \mathrm{E}-04$ & (6.55E-04) & $6.67 \mathrm{E}-02$ & $(6.50 \mathrm{E}-06)$ & (4.80E-05) \\
\hline B1PBB0A & C5602 & 92.3 & $(1.53 \mathrm{E}-01)$ & $1.83 \mathrm{E}-04$ & (6.82E-04) & $5.37 \mathrm{E}-02$ & $(2.00 \mathrm{E}-06)$ & (6.20E-05) \\
\hline B1PBB1B & C5606 & 51.8 & $(1.02 \mathrm{E}-02)$ & $5.37 \mathrm{E}-03$ & $(1.11 \mathrm{E}-03)$ & $2.07 \mathrm{E}-02$ & $(2.50 \mathrm{E}-06)$ & (3.53E-04) \\
\hline B1PBB1A & $\mathrm{C} 5606$ & 52.3 & $(1.02 \mathrm{E}-02)$ & $2.41 \mathrm{E}-02$ & $(1.42 \mathrm{E}-03)$ & 4.34E-02 & $(5.03 \mathrm{E}-06)$ & $(2.34 \mathrm{E}-04)$ \\
\hline B1PBB1A DUP & C5606 & 52.3 & $(2.04 \mathrm{E}-02)$ & $2.52 \mathrm{E}-02$ & $(1.16 \mathrm{E}-03)$ & $5.22 \mathrm{E}-02$ & $(2.00 \mathrm{E}-06)$ & (2.82E-04) \\
\hline B1PK51B & C5608 & 64.3 & 1.63E-01 & $3.15 \mathrm{E}-03$ & $6.51 \mathrm{E}-02$ & $2.02 \mathrm{E}-02$ & $(2.15 \mathrm{E}-05)$ & $2.35 \mathrm{E}-03$ \\
\hline B1PK51A & C5608 & 64.8 & 5.43E-02 & $2.67 \mathrm{E}-03$ & $5.57 \mathrm{E}-03$ & $1.40 \mathrm{E}-01$ & $(1.00 \mathrm{E}-06)$ & $(3.28 \mathrm{E}-04)$ \\
\hline B1PK52B & C5608 & 85.8 & $4.88 \mathrm{E}+01$ & $9.38 \mathrm{E}-04$ & $6.00 \mathrm{E}+00$ & $6.84 \mathrm{E}-02$ & $8.90 \mathrm{E}-05$ & $6.09 \mathrm{E}-04$ \\
\hline B1PK52A & C5608 & 86.3 & $4.19 \mathrm{E}+01$ & $2.73 \mathrm{E}-03$ & $5.23 \mathrm{E}+00$ & $7.06 \mathrm{E}-02$ & $(1.90 \mathrm{E}-05)$ & $(4.90 \mathrm{E}-04)$ \\
\hline B1PK53B & C5608 & 97.8 & $1.59 \mathrm{E}+01$ & $1.30 \mathrm{E}-01$ & $3.29 \mathrm{E}+00$ & $2.05 \mathrm{E}-01$ & $5.22 \mathrm{E}-05$ & $8.21 \mathrm{E}-04$ \\
\hline B1PK53A & C5608 & 98.3 & $1.05 E+01$ & $6.33 \mathrm{E}-02$ & $1.86 \mathrm{E}+00$ & $1.33 \mathrm{E}-01$ & $(1.25 \mathrm{E}-05)$ & $(2.07 \mathrm{E}-04)$ \\
\hline \multicolumn{9}{|c|}{$\begin{array}{l}\text { Bold values denote concentrations elevated above background. } \\
\text { Parentheses indicate reported value was less than the limit of quantification for the analysis. } \\
\text { Less-than values indicate the instrument returned a negative value. } \\
\text { NR indicates the information was not reported on the chain of custody. } \\
\text { Shaded cells indicate grab samples. }\end{array}$} \\
\hline
\end{tabular}




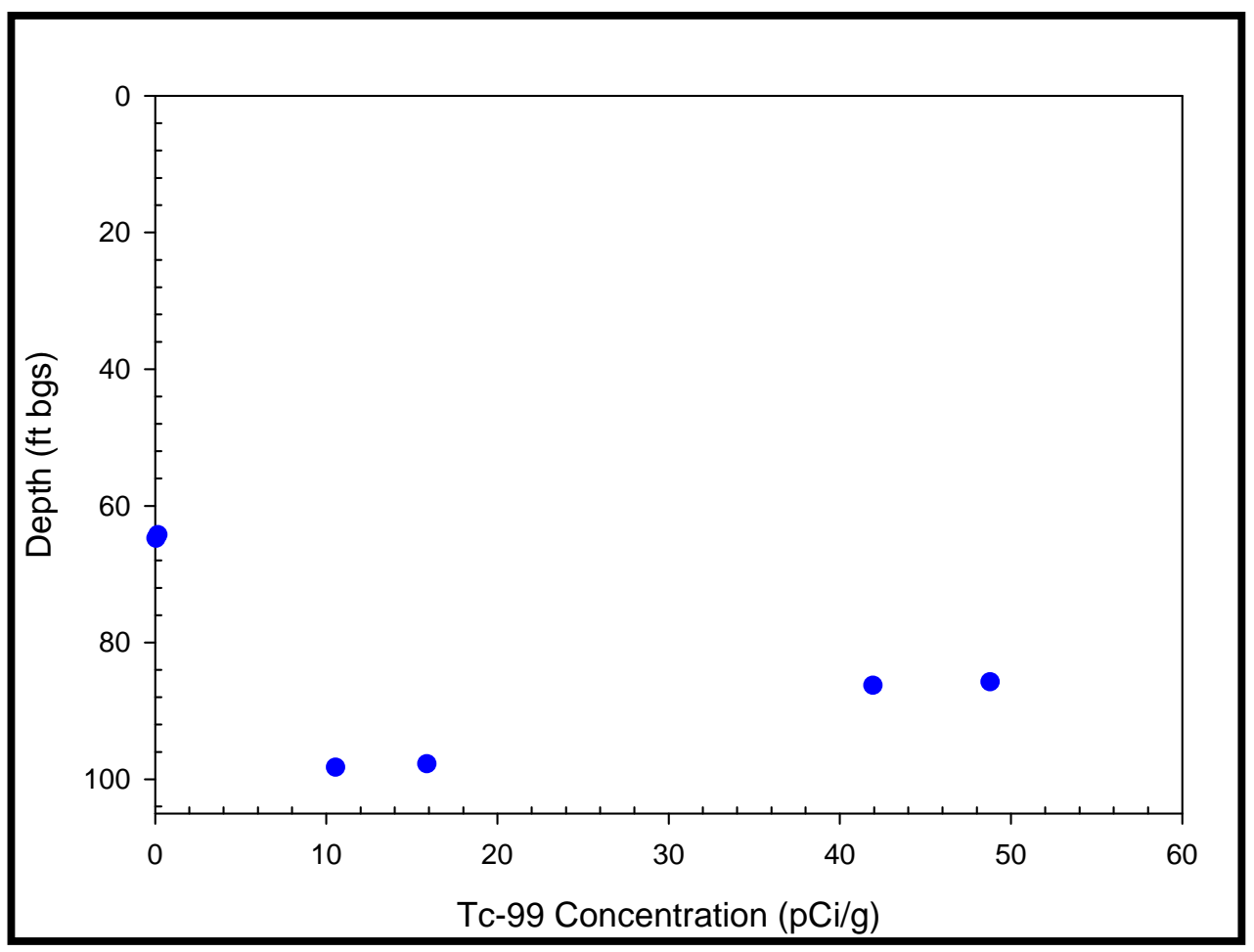

Figure 4.3. 1:1 Sediment:Water-Extractable Technetium-99 Data from Direct Push C5608 Samples

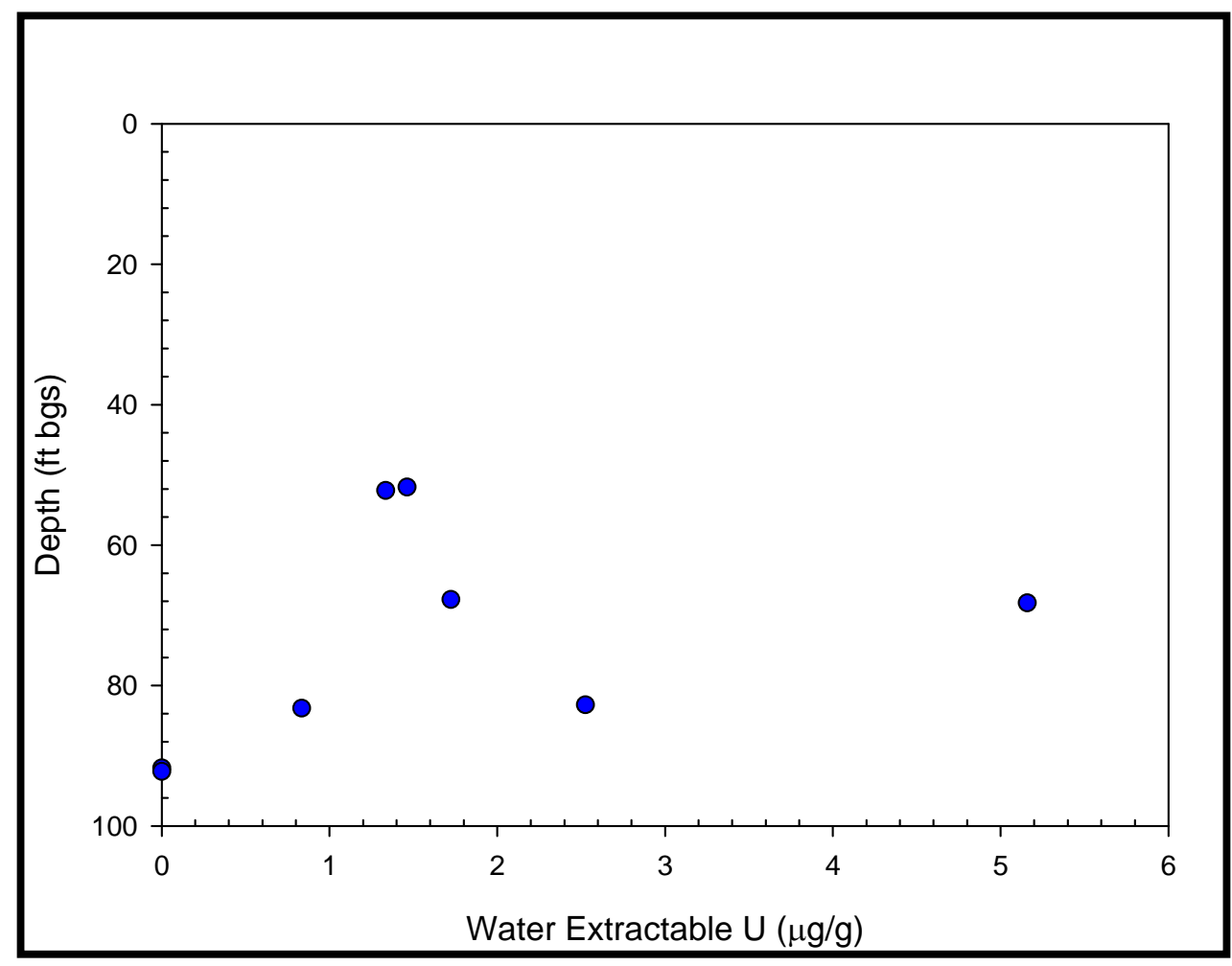

Figure 4.4. 1:1 Sediment:Water-Extractable Uranium-238 Data from Direct Push Hole C5602 Samples 


\subsubsection{Vadose Zone Porewater Chemical Composition}

The 1:1 water extract data was converted to derive the pore water composition of the vadose zone sediments so that electrical balances (anions vs. cation) of the samples could be performed. From knowledge of the moisture content of the sediment samples taken from the liners of each direct push sampler, the amount of de-ionized water that would be needed to make the water extract exactly one part water (total of native pore water and added de-ionized water) to one part by weight dry sediment was calculated. The ratio of the total volume of water in the extract to the native mass of pore water is the dilution factor. An assumption was made that the de-ionized water acted solely as a diluent of the existing pore water and that the de-ionized water did not dissolve any of the solids in the sediments. Thus by correcting for the dilution, an estimate of the actual chemical composition of the native pore-water in the vadose zone sediments could be derived.

The assumption that none of the solid is dissolved during the water extraction process is simplistic. In comparisons of actual vadose zone sediment pore water, which was obtained via ultracentrifugation of sediments, to the dilution-corrected calculated pore waters from both contaminated and uncontaminated sediments from the SX and B-BX Tank Farms (see Serne et al. 2002b, 2002c, 2002d, 2002e, 2002f), it was found that for highly contaminated sediments, the comparison is quite good. For slightly contaminated or uncontaminated sediments, the dilution-corrected water extract data is biased high by a factor of 2 to 7 for many constituents such that the true pore water is less saline. For the U Farm direct push data set, sufficient sample material was not available to enable the collection of actual pore water via ultracentrifugation. Therefore, it is assumed that the derived pore water concentrations for the U Farm direct push samples are slightly biased towards higher concentrations.

Tables 4.25 and 4.26 show the derived pore water composition of key constituents in meq/L. The six samples analyzed from push hole C5608 were the only sediment samples tested that contained significantly more dissolved salts (up to an order of magnitude more) than the rest of the samples analyzed as part of this study. The sample that contained the highest dissolved salt load was the deepest sample analyzed from push hole C5608. This sample, B1PK53A, which was collected from approximately $98 \mathrm{ft}$ bgs just south of tank $241-\mathrm{U}-110$, contained $210 \mathrm{meq} / \mathrm{L}$ cations vs. $208 \mathrm{meq} / \mathrm{L}$ dissolved anions. The cation chemistry of sample B1PK53A consisted almost entirely of sodium (203 $\mathrm{meq} / \mathrm{L})$, with trace amounts of calcium $(0.546 \mathrm{meq} / \mathrm{L})$ and magnesium $(0.125 \mathrm{meq} / \mathrm{L})$. The cation charge for this sample was compensated primarily by bicarbonate $(133 \mathrm{meq} / \mathrm{L})$ and nitrate $(60.9 \mathrm{meq} / \mathrm{L})$, with lesser amounts of sulfate $(6.82 \mathrm{meq} / \mathrm{l})$, fluoride $(5.48 \mathrm{meq} / \mathrm{L})$, chloride $(1.81 \mathrm{meq} / \mathrm{L})$, and chromate $(1.37$ $\mathrm{meq} / \mathrm{L})$. These concentrations are very dilute compared to the vadose zone pore waters found at the SX and BX tank farms, where the total dissolved salt loads were as high as 7,000 to 17,000 and 1,000 meq/L, respectively. The dissolved salt load in the C5608 push hole samples were more similar to those measured below tank T-106, which ranged from 200 to $250 \mathrm{meq} / \mathrm{L}$ each for cations and anions (total $\sim 450$ to $500 \mathrm{meq} / \mathrm{L})$.

Table 4.25. Calculated Pore Water Anion Concentrations in the U Tank Farm Core Samples

\begin{tabular}{||c|c|c|c|c|c|c|c|c||}
\hline $\begin{array}{c}\text { Sample } \\
\text { ID }\end{array}$ & $\begin{array}{c}\text { Probe } \\
\text { Hole } \\
\text { ID }\end{array}$ & $\begin{array}{c}\text { Mid- } \\
\text { Depth } \\
\text { ft bgs }\end{array}$ & $\begin{array}{c}\text { Fluoride } \\
\text { meq/L }\end{array}$ & $\begin{array}{c}\text { Chloride } \\
\text { meq/L }\end{array}$ & $\begin{array}{c}\text { Nitrate } \\
\text { meq/L }\end{array}$ & $\begin{array}{c}\text { Sulfate } \\
\text { meq/L }\end{array}$ & $\begin{array}{c}\text { Phosphate } \\
\text { meq/L }\end{array}$ & $\begin{array}{c}\text { Alkalinity } \\
\text { meq/L }\end{array}$ \\
\hline B1NDW3B & C5590 & 96.3 & $1.92 \mathrm{E}-01$ & $3.21 \mathrm{E}+00$ & $3.55 \mathrm{E}-01$ & $2.04 \mathrm{E}+00$ & $<5.95 \mathrm{E}-01$ & $1.22 \mathrm{E}+01$ \\
\hline
\end{tabular}




\begin{tabular}{|c|c|c|c|c|c|c|c|c|}
\hline $\begin{array}{c}\text { Sample } \\
\text { ID }\end{array}$ & $\begin{array}{c}\text { Probe } \\
\text { Hole } \\
\text { ID } \\
\end{array}$ & $\begin{array}{l}\text { Mid- } \\
\text { Depth } \\
\mathrm{ft} \text { bgs }\end{array}$ & $\begin{array}{c}\text { Fluoride } \\
\text { meq/L }\end{array}$ & $\begin{array}{c}\text { Chloride } \\
\text { meq/L }\end{array}$ & $\begin{array}{l}\text { Nitrate } \\
\mathrm{meq} / \mathrm{L}\end{array}$ & $\begin{array}{l}\text { Sulfate } \\
\mathrm{meq} / \mathrm{L}\end{array}$ & $\begin{array}{c}\text { Phosphate } \\
\text { meq/L }\end{array}$ & $\begin{array}{c}\text { Alkalinity } \\
\text { meq/L }\end{array}$ \\
\hline B1NDW3A & C5590 & 96.8 & $1.52 \mathrm{E}-01$ & $4.06 \mathrm{E}+00$ & $3.79 \mathrm{E}-01$ & $1.86 \mathrm{E}+00$ & $<4.31 \mathrm{E}-01$ & $9.26 \mathrm{E}+00$ \\
\hline B1NDW3A DUP & C5590 & 96.8 & $1.61 \mathrm{E}-01$ & $4.07 \mathrm{E}+00$ & $4.15 \mathrm{E}-01$ & $1.94 \mathrm{E}+00$ & $<4.31 \mathrm{E}-01$ & $9.13 \mathrm{E}+00$ \\
\hline B1NHV0B & C5592 & 62.3 & $2.69 \mathrm{E}-01$ & $1.64 \mathrm{E}-01$ & $8.40 \mathrm{E}-01$ & $1.70 \mathrm{E}+00$ & $<3.63 \mathrm{E}-01$ & $8.74 \mathrm{E}+00$ \\
\hline B1NHV0A & C5592 & 62.8 & $2.29 \mathrm{E}-01$ & $2.14 \mathrm{E}-01$ & $1.03 \mathrm{E}+00$ & $2.41 \mathrm{E}+00$ & $<6.01 \mathrm{E}-01$ & $1.41 \mathrm{E}+01$ \\
\hline B1NTC6B & C5598 & 50.3 & $5.35 \mathrm{E}-01$ & 4.19E-01 & $1.24 \mathrm{E}+00$ & 7.92E-01 & $<4.53 \mathrm{E}-01$ & $1.27 \mathrm{E}+01$ \\
\hline B1NTC6A & C5598 & 50.8 & $3.26 \mathrm{E}-01$ & 4.11E-01 & $1.91 \mathrm{E}+00$ & $1.47 \mathrm{E}+00$ & $<3.60 \mathrm{E}-01$ & $1.00 \mathrm{E}+01$ \\
\hline B1NTC7B & C5598 & 59.8 & $4.80 \mathrm{E}-01$ & 3.49E-01 & $9.02 \mathrm{E}-01$ & $3.63 \mathrm{E}+00$ & $<5.77 \mathrm{E}-01$ & $1.43 \mathrm{E}+01$ \\
\hline B1NTC7A & C5598 & 60.3 & $4.36 \mathrm{E}-01$ & $4.06 \mathrm{E}-01$ & 7.95E-01 & $3.16 \mathrm{E}+00$ & $<5.91 \mathrm{E}-01$ & $1.42 \mathrm{E}+01$ \\
\hline B1NTC8B & C5598 & 82.3 & $2.57 \mathrm{E}-01$ & $1.58 \mathrm{E}+00$ & $2.77 \mathrm{E}+00$ & $3.16 \mathrm{E}+00$ & $<2.94 \mathrm{E}-01$ & $7.36 \mathrm{E}+00$ \\
\hline B1NTC8B DUP & C5598 & 82.3 & $2.70 \mathrm{E}-01$ & $1.77 \mathrm{E}+00$ & $2.83 \mathrm{E}+00$ & $3.29 \mathrm{E}+00$ & $<2.95 \mathrm{E}-01$ & $6.63 \mathrm{E}+00$ \\
\hline B1NTC8A & C5598 & 82.8 & NR & $1.98 \mathrm{E}+00$ & $1.88 \mathrm{E}+00$ & $4.86 \mathrm{E}+00$ & $<5.28 \mathrm{E}-01$ & $1.37 \mathrm{E}+01$ \\
\hline B1NTC9B & C5596 & 50.8 & NR & $3.32 \mathrm{E}-01$ & $1.25 \mathrm{E}+00$ & $8.54 \mathrm{E}-01$ & $<7.85 \mathrm{E}-01$ & $2.17 \mathrm{E}+01$ \\
\hline B1NTC9A & C5596 & 51.3 & NR & $3.54 \mathrm{E}-01$ & $1.16 \mathrm{E}+00$ & $7.56 \mathrm{E}-01$ & $<9.26 \mathrm{E}-01$ & $2.41 \mathrm{E}+01$ \\
\hline B1NTD0B & C5596 & 61.3 & NR & $3.82 \mathrm{E}-01$ & $6.07 \mathrm{E}-01$ & $4.35 \mathrm{E}+00$ & $<6.08 \mathrm{E}-01$ & $1.48 \mathrm{E}+01$ \\
\hline B1NTD0A & C5596 & 61.8 & $7.56 \mathrm{E}-01$ & $5.54 \mathrm{E}-01$ & $<2.71 \mathrm{E}-01$ & $5.25 \mathrm{E}+00$ & $<7.95 \mathrm{E}-01$ & $2.02 \mathrm{E}+01$ \\
\hline B1NTD1B & C5596 & 77.8 & $5.44 \mathrm{E}-01$ & 7.97E-01 & $4.95 \mathrm{E}+00$ & $7.01 \mathrm{E}+00$ & $<8.28 \mathrm{E}-01$ & $1.81 \mathrm{E}+01$ \\
\hline B1NTD1B DUP & C5596 & 77.8 & $5.34 \mathrm{E}-01$ & $9.10 \mathrm{E}-01$ & $5.83 \mathrm{E}+00$ & $7.93 \mathrm{E}+00$ & $<7.44 \mathrm{E}-01$ & $1.55 \mathrm{E}+01$ \\
\hline B1NTD1A & C5596 & 78.3 & $5.12 \mathrm{E}-01$ & $1.04 \mathrm{E}+00$ & $4.92 \mathrm{E}+00$ & $8.73 \mathrm{E}+00$ & $<7.15 \mathrm{E}-01$ & $1.61 \mathrm{E}+01$ \\
\hline B1NTD2B & C5596 & 82.8 & NR & $4.09 \mathrm{E}+00$ & $7.49 \mathrm{E}+00$ & $6.48 \mathrm{E}+00$ & $<1.50 \mathrm{E}+00$ & $3.18 \mathrm{E}+01$ \\
\hline B1NTD2A & C5596 & 83.3 & NR & $4.23 \mathrm{E}+00$ & $7.14 \mathrm{E}+00$ & $6.32 \mathrm{E}+00$ & $<1.61 \mathrm{E}+00$ & $3.36 \mathrm{E}+01$ \\
\hline B1NTD3B & C5600 & 50.3 & NR & $1.92 \mathrm{E}-01$ & $2.45 \mathrm{E}+00$ & $1.37 \mathrm{E}+00$ & $<3.97 \mathrm{E}-01$ & $1.01 \mathrm{E}+01$ \\
\hline B1NTD3A & $\mathrm{C} 5600$ & 50.8 & NR & 4.84E-01 & $2.02 \mathrm{E}+00$ & $1.43 \mathrm{E}+00$ & $<4.81 \mathrm{E}-01$ & $1.19 \mathrm{E}+01$ \\
\hline B1NTD4B & C5600 & 60.3 & 7.13E-01 & $3.26 \mathrm{E}-01$ & $4.83 \mathrm{E}-01$ & $2.39 \mathrm{E}+00$ & $<9.80 \mathrm{E}-01$ & $2.42 \mathrm{E}+01$ \\
\hline B1NTD4A & C5600 & 60.8 & $3.93 \mathrm{E}-01$ & $<1.60 \mathrm{E}-01$ & 4.64E-01 & $1.73 \mathrm{E}+00$ & $<5.38 \mathrm{E}-01$ & $1.28 \mathrm{E}+01$ \\
\hline B1NTD5B & C5600 & 82.3 & $1.27 \mathrm{E}-01$ & $1.69 \mathrm{E}+00$ & $1.48 \mathrm{E}+00$ & $5.07 \mathrm{E}+00$ & $<3.02 \mathrm{E}-01$ & $8.33 \mathrm{E}+00$ \\
\hline B1NTD5A & $\mathrm{C} 5600$ & 82.8 & $7.58 \mathrm{E}-01$ & $2.02 \mathrm{E}+00$ & $<2.91 \mathrm{E}-01$ & $6.40 \mathrm{E}+00$ & $<8.55 \mathrm{E}-01$ & $2.11 \mathrm{E}+01$ \\
\hline B1P1K6B & $\mathrm{C} 5600$ & 88.8 & NR & $5.00 \mathrm{E}+00$ & $2.42 \mathrm{E}+00$ & $6.32 \mathrm{E}+00$ & $<1.19 \mathrm{E}+00$ & $2.64 \mathrm{E}+01$ \\
\hline B1P1K6A & C5600 & 89.3 & NR & $4.29 \mathrm{E}+00$ & $1.86 \mathrm{E}+00$ & $4.97 \mathrm{E}+00$ & $<5.97 \mathrm{E}-01$ & $1.32 \mathrm{E}+01$ \\
\hline B1P3F9B & C5604 & 50.8 & NR & $1.25 \mathrm{E}+00$ & 4.79E-01 & $8.72 \mathrm{E}-01$ & $<3.50 \mathrm{E}-01$ & $1.07 \mathrm{E}+01$ \\
\hline B1P3F9A & C5604 & 51.3 & NR & $9.93 \mathrm{E}-02$ & $2.81 \mathrm{E}-01$ & 7.94E-01 & $<3.18 \mathrm{E}-01$ & $8.66 \mathrm{E}+00$ \\
\hline B1P3H0B & $\mathrm{C} 5602$ & 51.8 & NR & 3.83E-01 & $1.01 \mathrm{E}+00$ & $1.23 \mathrm{E}+00$ & $<6.29 \mathrm{E}-01$ & $1.59 \mathrm{E}+01$ \\
\hline B1P3H0A & C5602 & 52.3 & NR & 5.03E-01 & $7.55 \mathrm{E}-01$ & $1.28 \mathrm{E}+00$ & $<1.71 \mathrm{E}+00$ & $4.12 \mathrm{E}+01$ \\
\hline B1P3H1B & $\mathrm{C} 5602$ & 67.8 & NR & $4.20 \mathrm{E}-01$ & $9.62 \mathrm{E}-01$ & $3.67 \mathrm{E}+00$ & $<1.24 \mathrm{E}+00$ & $2.67 \mathrm{E}+01$ \\
\hline B1P3H1A & C5602 & 68.3 & NR & $2.55 \mathrm{E}-01$ & $9.27 \mathrm{E}-01$ & $2.86 \mathrm{E}+00$ & $<4.92 \mathrm{E}-01$ & $1.37 \mathrm{E}+01$ \\
\hline B1P3H2B & $\mathrm{C} 5602$ & 82.8 & NR & 4.12E-01 & $1.27 \mathrm{E}+00$ & $5.26 \mathrm{E}+00$ & $<4.97 \mathrm{E}-01$ & $1.28 \mathrm{E}+01$ \\
\hline B1P3H2A & $\mathrm{C} 5602$ & 83.3 & NR & $3.68 \mathrm{E}-01$ & $1.06 \mathrm{E}+00$ & $4.92 \mathrm{E}+00$ & $<1.07 \mathrm{E}+00$ & $2.20 \mathrm{E}+01$ \\
\hline B1PBB0B & $\mathrm{C} 5602$ & 91.8 & NR & 7.38E-01 & $4.56 \mathrm{E}+00$ & $7.63 \mathrm{E}+00$ & $<6.06 \mathrm{E}-01$ & $1.13 \mathrm{E}+01$ \\
\hline B1PBB0A & $\mathrm{C} 5602$ & 92.3 & NR & 7.33E-01 & $2.75 \mathrm{E}+00$ & $7.06 \mathrm{E}+00$ & $<7.72 \mathrm{E}-01$ & $1.46 \mathrm{E}+01$ \\
\hline B1PBB1B & C5606 & 51.8 & $1.10 \mathrm{E}-01$ & $1.98 \mathrm{E}-01$ & $1.88 \mathrm{E}+00$ & $3.60 \mathrm{E}+00$ & $<2.71 \mathrm{E}-01$ & $5.14 \mathrm{E}+00$ \\
\hline B1PBB1A & C5606 & 52.3 & $1.26 \mathrm{E}-01$ & $3.02 \mathrm{E}-01$ & $1.77 \mathrm{E}+00$ & $3.37 \mathrm{E}+00$ & $<2.46 \mathrm{E}-01$ & $5.52 \mathrm{E}+00$ \\
\hline B1PBB1A DUP & C5606 & 52.3 & $1.22 \mathrm{E}-01$ & $2.80 \mathrm{E}-01$ & $1.64 \mathrm{E}+00$ & $3.25 \mathrm{E}+00$ & $<2.45 \mathrm{E}-01$ & $5.82 \mathrm{E}+00$ \\
\hline B1PK51B & C5608 & 64.3 & 7.15E-01 & $1.31 \mathrm{E}-01$ & 7.16E-01 & $1.59 \mathrm{E}+00$ & $<2.64 \mathrm{E}-01$ & $4.47 \mathrm{E}+01$ \\
\hline
\end{tabular}




\begin{tabular}{|c|c|c|c|c|c|c|c|c|}
\hline $\begin{array}{l}\text { Sample } \\
\text { ID }\end{array}$ & $\begin{array}{c}\text { Probe } \\
\text { Hole } \\
\text { ID }\end{array}$ & $\begin{array}{l}\text { Mid- } \\
\text { Depth } \\
\text { ft bgs }\end{array}$ & $\begin{array}{l}\text { Fluoride } \\
\text { meq/L }\end{array}$ & $\begin{array}{c}\text { Chloride } \\
\text { meq/L }\end{array}$ & $\begin{array}{l}\text { Nitrate } \\
\mathrm{meq} / \mathrm{L}\end{array}$ & $\begin{array}{l}\text { Sulfate } \\
\mathrm{meq} / \mathrm{L}\end{array}$ & $\begin{array}{l}\text { Phosphate } \\
\text { meq/L }\end{array}$ & $\begin{array}{c}\text { Alkalinity } \\
\text { meq/L }\end{array}$ \\
\hline B1PK51A & C5608 & 64.8 & $1.10 \mathrm{E}+00$ & $2.54 \mathrm{E}-01$ & $<2.21 \mathrm{E}-01$ & $1.73 \mathrm{E}+00$ & $<<6.48 \mathrm{E}-01$ & $9.36 E+01$ \\
\hline B1PK52B & C5608 & 85.8 & $4.90 \mathrm{E}+00$ & $1.51 \mathrm{E}+00$ & $4.78 \mathrm{E}+01$ & $3.55 \mathrm{E}+00$ & 4.14E-01 & $9.01 E+01$ \\
\hline B1PK52A & C5608 & 86.3 & $4.22 \mathrm{E}+00$ & $1.33 \mathrm{E}+00$ & $4.54 \mathrm{E}+01$ & $3.46 \mathrm{E}+00$ & $4.85 \mathrm{E}-01$ & $1.04 E+02$ \\
\hline B1PK53B & C5608 & 97.8 & $6.74 \mathrm{E}+00$ & $1.89 \mathrm{E}+00$ & $6.06 \mathrm{E}+01$ & $6.72 \mathrm{E}+00$ & $4.48 \mathrm{E}-01$ & $6.58 \mathrm{E}+01$ \\
\hline B1PK53A & C5608 & 98.3 & $5.48 \mathrm{E}+00$ & $1.81 \mathrm{E}+00$ & $6.09 \mathrm{E}+01$ & $6.82 \mathrm{E}+00$ & $<9.28 \mathrm{E}-01$ & $1.33 E+02$ \\
\hline
\end{tabular}

Table 4.26. Calculated Pore Water Cation Concentrations in the U Tank Farm Direct Push Core Samples

\begin{tabular}{|c|c|c|c|c|c|c|}
\hline $\begin{array}{c}\text { Sample } \\
\text { ID }\end{array}$ & $\begin{array}{c}\text { Probe Hole } \\
\text { ID }\end{array}$ & $\begin{array}{c}\text { Mid-Depth } \\
\mathrm{ft} \text { bgs }\end{array}$ & $\begin{array}{c}\text { Calcium } \\
\mathrm{meq} / \mathrm{L}\end{array}$ & $\begin{array}{c}\text { Potassium } \\
\text { meq/L }\end{array}$ & $\begin{array}{c}\text { Magnesium } \\
\text { meq/L }\end{array}$ & $\begin{array}{l}\text { Sodium } \\
\text { meq/L }\end{array}$ \\
\hline B1NDW3B & C5590 & 96.3 & $5.97 \mathrm{E}+00$ & $1.02 \mathrm{E}+00$ & $2.06 \mathrm{E}+00$ & $7.23 \mathrm{E}+00$ \\
\hline B1NDW3A & C5590 & 96.8 & $5.12 \mathrm{E}+00$ & $8.12 \mathrm{E}-01$ & $1.76 \mathrm{E}+00$ & $6.37 \mathrm{E}+00$ \\
\hline B1NDW3A DUP & C5590 & 96.8 & $5.37 \mathrm{E}+00$ & $8.29 \mathrm{E}-01$ & $1.84 \mathrm{E}+00$ & $6.53 \mathrm{E}+00$ \\
\hline B1NHV0B & C5592 & 62.3 & $2.01 \mathrm{E}+00$ & $7.85 \mathrm{E}-01$ & $1.20 \mathrm{E}+00$ & $8.16 \mathrm{E}+00$ \\
\hline B1NHV0A & C5592 & 62.8 & $3.13 \mathrm{E}+00$ & $1.27 \mathrm{E}+00$ & $1.85 \mathrm{E}+00$ & $1.09 \mathrm{E}+01$ \\
\hline B1NTC6B & C5598 & 50.3 & $3.01 \mathrm{E}+00$ & 9.34E-01 & $1.21 \mathrm{E}+00$ & $8.44 \mathrm{E}+00$ \\
\hline B1NTC6A & C5598 & 50.8 & $3.42 \mathrm{E}+00$ & $8.18 \mathrm{E}-01$ & $1.38 \mathrm{E}+00$ & $6.42 \mathrm{E}+00$ \\
\hline B1NTC7B & C5598 & 59.8 & $5.26 \mathrm{E}+00$ & $1.49 \mathrm{E}+00$ & $2.88 \mathrm{E}+00$ & $8.45 \mathrm{E}+00$ \\
\hline B1NTC7A & C5598 & 60.3 & $4.63 \mathrm{E}+00$ & $1.56 \mathrm{E}+00$ & $2.47 \mathrm{E}+00$ & $8.34 \mathrm{E}+00$ \\
\hline B1NTC8B & C5598 & 82.3 & $4.91 \mathrm{E}+00$ & $7.88 \mathrm{E}-01$ & $2.50 \mathrm{E}+00$ & $4.89 \mathrm{E}+00$ \\
\hline B1NTC8B DUP & C5598 & 82.3 & $4.88 \mathrm{E}+00$ & $8.09 \mathrm{E}-01$ & $2.45 \mathrm{E}+00$ & $5.08 \mathrm{E}+00$ \\
\hline B1NTC8A & C5598 & 82.8 & $6.48 \mathrm{E}+00$ & $1.60 \mathrm{E}+00$ & $3.27 \mathrm{E}+00$ & $8.66 \mathrm{E}+00$ \\
\hline B1NTC9B & C5596 & 50.8 & $3.57 \mathrm{E}+00$ & $1.47 \mathrm{E}+00$ & $1.78 \mathrm{E}+00$ & $1.54 \mathrm{E}+01$ \\
\hline B1NTC9A & C5596 & 51.3 & $4.17 \mathrm{E}+00$ & $1.71 \mathrm{E}+00$ & $2.13 \mathrm{E}+00$ & $1.60 \mathrm{E}+01$ \\
\hline B1NTD0B & C5596 & 61.3 & $5.79 \mathrm{E}+00$ & $1.79 \mathrm{E}+00$ & $3.44 \mathrm{E}+00$ & $7.77 \mathrm{E}+00$ \\
\hline B1NTD0A & C5596 & 61.8 & $6.69 \mathrm{E}+00$ & $2.56 \mathrm{E}+00$ & $3.94 \mathrm{E}+00$ & $1.09 \mathrm{E}+01$ \\
\hline B1NTD1B & C5596 & 77.8 & $9.97 \mathrm{E}+00$ & $2.11 \mathrm{E}+00$ & $4.08 \mathrm{E}+00$ & $1.13 \mathrm{E}+01$ \\
\hline B1NTD1B DUP & C5596 & 77.8 & $1.02 \mathrm{E}+01$ & $2.03 \mathrm{E}+00$ & $4.26 \mathrm{E}+00$ & $1.15 \mathrm{E}+01$ \\
\hline B1NTD1A & C5596 & 78.3 & $1.00 \mathrm{E}+01$ & $2.09 \mathrm{E}+00$ & $4.19 \mathrm{E}+00$ & $1.15 \mathrm{E}+01$ \\
\hline B1NTD2B & C5596 & 82.8 & $1.38 \mathrm{E}+01$ & $4.50 \mathrm{E}+00$ & $7.84 \mathrm{E}+00$ & $2.05 \mathrm{E}+01$ \\
\hline B1NTD2A & C5596 & 83.3 & $1.40 \mathrm{E}+01$ & $4.13 \mathrm{E}+00$ & $7.64 \mathrm{E}+00$ & $2.08 \mathrm{E}+01$ \\
\hline B1NTD3B & C5600 & 50.3 & $3.77 \mathrm{E}+00$ & $7.76 \mathrm{E}-01$ & $1.49 \mathrm{E}+00$ & $5.33 \mathrm{E}+00$ \\
\hline B1NTD3A & C5600 & 50.8 & $4.32 \mathrm{E}+00$ & $9.93 \mathrm{E}-01$ & $1.55 \mathrm{E}+00$ & $7.11 \mathrm{E}+00$ \\
\hline B1NTD4B & C5600 & 60.3 & $7.58 \mathrm{E}+00$ & $2.95 \mathrm{E}+00$ & $4.07 \mathrm{E}+00$ & $1.19 \mathrm{E}+01$ \\
\hline B1NTD4A & C5600 & 60.8 & $4.53 \mathrm{E}+00$ & $1.48 \mathrm{E}+00$ & $2.36 \mathrm{E}+00$ & $6.35 \mathrm{E}+00$ \\
\hline B1NTD5B & C5600 & 82.3 & $5.49 \mathrm{E}+00$ & $1.20 \mathrm{E}+00$ & $2.76 \mathrm{E}+00$ & $6.92 \mathrm{E}+00$ \\
\hline B1NTD5A & C5600 & 82.8 & $8.49 \mathrm{E}+00$ & $2.74 \mathrm{E}+00$ & $3.96 \mathrm{E}+00$ & $1.42 \mathrm{E}+01$ \\
\hline B1P1K6B & C5600 & 88.8 & $1.20 \mathrm{E}+01$ & $2.57 \mathrm{E}+00$ & $4.83 \mathrm{E}+00$ & $1.59 \mathrm{E}+01$ \\
\hline B1P1K6A & C5600 & 89.3 & $8.35 \mathrm{E}+00$ & $1.38 \mathrm{E}+00$ & $3.29 \mathrm{E}+00$ & $9.43 \mathrm{E}+00$ \\
\hline B1P3F9B & C5604 & 50.8 & $2.25 \mathrm{E}+00$ & $7.08 \mathrm{E}-01$ & $1.09 \mathrm{E}+00$ & $8.09 \mathrm{E}+00$ \\
\hline B1P3F9A & C5604 & 51.3 & $1.35 \mathrm{E}+00$ & 5.44E-01 & $7.20 \mathrm{E}-01$ & $5.58 \mathrm{E}+00$ \\
\hline B1P3H0B & $\mathrm{C} 5602$ & 51.8 & $3.61 \mathrm{E}+00$ & $1.24 \mathrm{E}+00$ & $2.19 \mathrm{E}+00$ & $9.58 \mathrm{E}+00$ \\
\hline
\end{tabular}




\begin{tabular}{|c|c|c|c|c|c|c|}
\hline $\begin{array}{l}\text { Sample } \\
\text { ID }\end{array}$ & $\begin{array}{l}\text { Probe Hole } \\
\text { ID }\end{array}$ & $\begin{array}{l}\text { Mid-Depth } \\
\text { ft bgs }\end{array}$ & $\begin{array}{c}\begin{array}{c}\text { Calcium } \\
\text { meq/L }\end{array} \\
\text {. }\end{array}$ & $\begin{array}{c}\text { Potassium } \\
\text { meq/L }\end{array}$ & $\begin{array}{l}\text { Magnesium } \\
\text { meq/L }\end{array}$ & $\begin{array}{l}\begin{array}{l}\text { Sodium } \\
\text { meq/L }\end{array} \\
\end{array}$ \\
\hline B1P3H0A & C5602 & "52.3 & $7.85 \mathrm{E}+00$ & $2.70 \mathrm{E}+00$ & $5.42 \mathrm{E}+00$ & $2.05 \mathrm{E}+01$ \\
\hline B1P3H1B & C5602 & 67.8 & $8.34 \mathrm{E}+00$ & $2.53 \mathrm{E}+00$ & $4.69 \mathrm{E}+00$ & $1.23 \mathrm{E}+01$ \\
\hline B1P3H1A & C5602 & 68.3 & $4.79 \mathrm{E}+00$ & $1.03 \mathrm{E}+00$ & $2.42 \mathrm{E}+00$ & $6.04 \mathrm{E}+00$ \\
\hline B1P3H2B & $\mathrm{C} 5602$ & 82.8 & $5.70 \mathrm{E}+00$ & $1.50 \mathrm{E}+00$ & $2.63 \mathrm{E}+00$ & $8.47 \mathrm{E}+00$ \\
\hline B1P3H2A & C5602 & 83.3 & $8.00 \mathrm{E}+00$ & $2.34 \mathrm{E}+00$ & $3.67 \mathrm{E}+00$ & $1.07 \mathrm{E}+01$ \\
\hline B1PBB0B & C5602 & 91.8 & $8.45 \mathrm{E}+00$ & $1.40 \mathrm{E}+00$ & $2.91 \mathrm{E}+00$ & $8.71 \mathrm{E}+00$ \\
\hline B1PBB0A & C5602 & 92.3 & $7.93 \mathrm{E}+00$ & $1.62 \mathrm{E}+00$ & $2.78 \mathrm{E}+00$ & $9.51 \mathrm{E}+00$ \\
\hline B1PBB1B & C5606 & 51.8 & $3.96 \mathrm{E}+00$ & $6.27 \mathrm{E}-01$ & $1.46 \mathrm{E}+00$ & $4.62 \mathrm{E}+00$ \\
\hline B1PBB1A & C5606 & 52.3 & $3.95 \mathrm{E}+00$ & $6.77 \mathrm{E}-01$ & $1.53 \mathrm{E}+00$ & $4.63 \mathrm{E}+00$ \\
\hline B1PBB1A DUP & C5606 & 52.3 & $3.71 \mathrm{E}+00$ & $6.93 \mathrm{E}-01$ & $1.48 \mathrm{E}+00$ & $4.55 \mathrm{E}+00$ \\
\hline B1PK51B & C5608 & 64.3 & $1.61 E-01$ & $(2.43 E-01)$ & $(4.81 E-02)$ & $4.64 \mathrm{E}+01$ \\
\hline B1PK51A & C5608 & 64.8 & $3.28 E-01$ & $(5.77 E-01)$ & $(8.72 E-02)$ & $8.51 \mathrm{E}+01$ \\
\hline B1PK52B & C5608 & 85.8 & $2.02 E-01$ & $(4.21 E-01)$ & $(4.44 E-02)$ & $1.54 \mathrm{E}+02$ \\
\hline B1PK52A & C5608 & 86.3 & $1.83 E-01$ & $(3.50 E-01)$ & $(1.29 E-02)$ & $1.60 \mathrm{E}+02$ \\
\hline B1PK53B & C5608 & 97.8 & $2.76 E-01$ & $(5.48 E-01)$ & $(9.29 E-02)$ & $1.47 \mathrm{E}+02$ \\
\hline B1PK53A & C5608 & 98.3 & $5.46 E-01$ & $(8.16 E-01)$ & $(1.25 E-01)$ & $2.03 E+02$ \\
\hline
\end{tabular}

The remaining samples contained relatively low dissolved salt loads, which ranged from a low of 18.2 meq/L total (anions and cations) for sample B1P3F9A (north of tank 241-U-201) to a high of $98.3 \mathrm{meq} / \mathrm{L}$ for sample B1NTD2A (north of tank 241-U-105). The fact that the calculated porewater concentrations for the samples collected north of the 241-U-201 tank were dilute with respect to dissolved salts does not correlate well with the electrical resistivity data reported by Rucker et al. (2006), which showed a zone of elevated conductivity near that location. However, it must be noted that the samples collected from all of the direct push holes were from discrete depths and only represent a "snapshot" of the vadose zone. In order to more thoroughly evaluate the correlation between field-collected resistivity data and actual laboratory-derived data, a more comprehensive set of samples would need to be analyzed.

Overall, the calculated charge balance between cations and anions for all of the samples was quite good (less than 15\% difference for most of the samples analyzed). However, samples B1NTD3B and B1P3H1A both contained approximately $20 \%$ less dissolved cations than anions. Based on evaluation of this data, it appears that either the bicarbonate measurement for these samples is biased high, or analyses have not accounted for a dissolved metal that is present in sufficient quantity to properly balance the electrical charge of these samples.

Sodium was present as the dominant cation (or co-dominant cation) in all of the samples analyzed. Sodium was also present as the dominant water-extractable cation in most of the samples from the background borehole. The lack of samples containing calcium as the dominant cation from all of these sampling locations indicates that the samples in this region have been impacted by a sodium-bearing waste fluid. The source(s) appears to be a moderately concentrated sodium-bearing waste solution that has displaced the natural divalent cations from the sediment cation exchange sites in the sediments. The total vertical (and lateral) extent of the ion exchange front is unknown due to the lack of sediment samples from deeper in the vadose zone. 
The porewater calculated concentrations of key contaminants of concern are presented in Table 4.27. The porewater corrected technetium-99 activity calculated in the samples collected from push hole C5608 ranged from just above the current maximum contaminant level (MCL) for drinking water $(908 \mathrm{pCi} / \mathrm{L}$ in sample B1PK51B) to $250,000 \mathrm{pCi} / \mathrm{L}$ in sample B1PK52B. While a technetium-99 activity of 250,000 $\mathrm{pCi} / \mathrm{L}$ is well above the MCL, it must be noted that this is a porewater corrected activity; the total activity in these samples will be diluted significantly once the porewater mixes with groundwater underlying the U Tank Farm. In addition to technetium-99, the samples retrieved from push hole C5608 also contained significantly elevated porewater concentrations of chromium. As noted by Wood and Jones (2003), tank 241-U-110 is estimated to have released more than $25 \mathrm{~kg}$ of chromium to the vadose zone; it appears fairly certain that push hole C5608 has intercepted the waste plume leaked from tank 241-U-110.

Push hole C5602 was the only other sample location to provide sediments with elevated porewater concentrations for key contaminants of concern. Namely, technetium-99 was found to be elevated in one sample from C5602, while uranium-238 was found to be elevated in several of the C5602 samples. Although a porewater corrected technetium-99 activity of $2500 \mathrm{pCi} / \mathrm{L}$ is well above the MCL, it does represent a trace amount of contamination in the vadose zone. Conversely, the peak porewater corrected uranium-238 concentration measured in the sediments retrieved from push hole C5602 was 53,600 $\mu \mathrm{g} / \mathrm{L}$, or nearly 2000 times the MCL for uranium $(30 \mu \mathrm{g} / \mathrm{L})$. Wood and Jones (2003) estimate that tank 241-U104 leaked $1.72 \mathrm{Ci}(5100 \mathrm{~kg})$ of uranium to the vadose zone. Therefore, it was not surprising to find a significant amount of contaminant uranium in a push hole emplaced between tanks 241-U-104 and 241U-105.

Table 4.27. Calculated Pore Water Mobile Metal Concentrations of Key Contaminants of Concern in the U Tank Farm Direct Push Samples

\begin{tabular}{||l|c|c|c|c|c|c||}
\hline $\begin{array}{c}\text { Sample } \\
\text { ID }\end{array}$ & $\begin{array}{c}\text { Probe } \\
\text { Hole ID }\end{array}$ & $\begin{array}{c}\text { Mid-Depth } \\
\mathrm{ft} \text { bgs }\end{array}$ & $\begin{array}{c}\text { Technetium-99 } \\
\mathrm{pCi} / \mathrm{L}\end{array}$ & $\begin{array}{c}\text { Uranium-238 } \\
\mu \mathrm{g} / \mathrm{L}\end{array}$ & $\begin{array}{c}\text { Chromium } \\
\mu \mathrm{g} / \mathrm{L}\end{array}$ & $\begin{array}{c}\text { Molybdenum } \\
\mu \mathrm{g} / \mathrm{L}\end{array}$ \\
\hline \hline B1NDW3B & C5590 & 96.3 & $(1.28 \mathrm{E}+02)$ & $4.91 \mathrm{E}+00$ & $(2.57 \mathrm{E}+01)$ & $5.74 \mathrm{E}+02$ \\
\hline B1NDW3A & C5590 & 96.8 & $(1.54 \mathrm{E}+02)$ & $4.04 \mathrm{E}+00$ & $2.40 \mathrm{E}+01$ & $7.09 \mathrm{E}+02$ \\
\hline B1NDW3A DUP & C5590 & 96.8 & $<1.54 \mathrm{E}+03$ & $4.26 \mathrm{E}+00$ & $2.52 \mathrm{E}+01$ & $6.81 \mathrm{E}+02$ \\
\hline B1NHV0B & C5592 & 62.3 & $<1.30 \mathrm{E}+03$ & $9.07 \mathrm{E}+00$ & $(1.23 \mathrm{E}+01)$ & $2.56 \mathrm{E}+02$ \\
\hline B1NHV0A & C5592 & 62.8 & $<2.15 \mathrm{E}+03$ & $1.09 \mathrm{E}+01$ & $(1.53 \mathrm{E}+01)$ & $3.88 \mathrm{E}+02$ \\
\hline B1NTC6B & C5598 & 50.3 & $<1.62 \mathrm{E}+03$ & $1.14 \mathrm{E}+01$ & $(1.44 \mathrm{E}+01)$ & $5.76 \mathrm{E}+02$ \\
\hline B1NTC6A & C5598 & 50.8 & $<1.29 \mathrm{E}+03$ & $6.32 \mathrm{E}+00$ & $(4.88 \mathrm{E}+00)$ & $3.42 \mathrm{E}+02$ \\
\hline B1NTC7B & C5598 & 59.8 & $<2.07 \mathrm{E}+03$ & $5.67 \mathrm{E}+00$ & $(1.47 \mathrm{E}+01)$ & $6.36 \mathrm{E}+02$ \\
\hline B1NTC7A & C5598 & 60.3 & $<2.11 \mathrm{E}+03$ & $3.81 \mathrm{E}+00$ & $(8.45 \mathrm{E}+00)$ & $6.96 \mathrm{E}+02$ \\
\hline B1NTC8B & C5598 & 82.3 & $<1.05 \mathrm{E}+03$ & $4.69 \mathrm{E}+00$ & $(1.42 \mathrm{E}+01)$ & $2.53 \mathrm{E}+02$ \\
\hline B1NTC8B DUP & C5598 & 82.3 & $<1.06 \mathrm{E}+03$ & $4.50 \mathrm{E}+00$ & $(1.47 \mathrm{E}+01)$ & $2.57 \mathrm{E}+02$ \\
\hline B1NTC8A & C5598 & 82.8 & $(1.14 \mathrm{E}+02)$ & $5.36 \mathrm{E}+00$ & $(2.19 \mathrm{E}+01)$ & $8.06 \mathrm{E}+02$ \\
\hline B1NTC9B & C5596 & 50.8 & $<2.81 \mathrm{E}+03$ & $2.15 \mathrm{E}+01$ & $(1.02 \mathrm{E}+01)$ & $4.08 \mathrm{E}+02$ \\
\hline B1NTC9A & C5596 & 51.3 & $<3.32 \mathrm{E}+03$ & $1.87 \mathrm{E}+01$ & $(1.26 \mathrm{E}+01)$ & $4.88 \mathrm{E}+02$ \\
\hline B1NTD0B & C5596 & 61.3 & $<2.18 \mathrm{E}+03$ & $7.57 \mathrm{E}+00$ & $(2.04 \mathrm{E}+01)$ & $1.02 \mathrm{E}+03$ \\
\hline B1NTD0A & C5596 & 61.8 & $<2.85 \mathrm{E}+03$ & $6.10 \mathrm{E}+00$ & $(1.88 \mathrm{E}+01)$ & $2.67 \mathrm{E}+03$ \\
\hline B1NTD1B & C5596 & 77.8 & $(9.49 \mathrm{E}+02)$ & $6.76 \mathrm{E}+00$ & $(3.66 \mathrm{E}+01)$ & $9.94 \mathrm{E}+02$ \\
\hline B1NTD1B DUP & C5596 & 77.8 & $(2.61 \mathrm{E}+03)$ & $7.01 \mathrm{E}+00$ & $(3.74 \mathrm{E}+01)$ & $1.02 \mathrm{E}+03$ \\
\hline B1NTD1A & C5596 & 78.3 & $(1.18 \mathrm{E}+03)$ & $6.67 \mathrm{E}+00$ & $5.27 \mathrm{E}+01$ & $1.16 \mathrm{E}+03$ \\
\hline
\end{tabular}




\begin{tabular}{|c|c|c|c|c|c|c|}
\hline $\begin{array}{c}\text { Sample } \\
\text { ID }\end{array}$ & $\begin{array}{c}\text { Probe } \\
\text { Hole ID }\end{array}$ & $\begin{array}{l}\text { Mid-Depth } \\
\mathrm{ft} \text { bgs }\end{array}$ & $\begin{array}{c}\text { Technetium-99 } \\
\text { pCi/L }\end{array}$ & $\begin{array}{c}\text { Uranium-238 } \\
\mu \mathrm{g} / \mathrm{L}\end{array}$ & $\begin{array}{c}\text { Chromium } \\
\mu \mathrm{g} / \mathrm{L}\end{array}$ & $\begin{array}{c}\text { Molybdenum } \\
\mu \mathrm{g} / \mathrm{L}\end{array}$ \\
\hline B1NTD2B & C5596 & 82.8 & 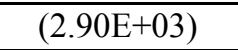 & $7.03 \mathrm{E}+00$ & 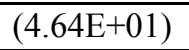 & $1.18 \mathrm{E}+03$ \\
\hline B1NTD2A & C5596 & 83.3 & $(4.04 \mathrm{E}+03)$ & $6.42 \mathrm{E}+00$ & $(3.54 \mathrm{E}+01)$ & $6.49 \mathrm{E}+02$ \\
\hline B1NTD3B & C5600 & 50.3 & $<1.42 \mathrm{E}+03$ & $8.56 \mathrm{E}+00$ & $(5.07 \mathrm{E}+00)$ & $2.61 \mathrm{E}+02$ \\
\hline B1NTD3A & C5600 & 50.8 & $(1.03 \mathrm{E}+02)$ & $8.04 \mathrm{E}+00$ & $(5.20 \mathrm{E}+00)$ & $1.10 \mathrm{E}+03$ \\
\hline B1NTD4B & C5600 & 60.3 & $<3.51 \mathrm{E}+03$ & $7.72 \mathrm{E}+00$ & $5.65 \mathrm{E}+01$ & $1.58 \mathrm{E}+03$ \\
\hline B1NTD4A & C5600 & 60.8 & $<1.93 \mathrm{E}+03$ & $4.14 \mathrm{E}+00$ & $3.02 \mathrm{E}+01$ & $5.53 \mathrm{E}+02$ \\
\hline B1NTD5B & C5600 & 82.3 & $(1.08 \mathrm{E}+02)$ & $5.68 \mathrm{E}+00$ & $1.99 \mathrm{E}+01$ & $1.00 \mathrm{E}+03$ \\
\hline B1NTD5A & C5600 & 82.8 & $(1.84 \mathrm{E}+02)$ & $5.47 \mathrm{E}+00$ & $4.65 \mathrm{E}+01$ & $2.33 \mathrm{E}+03$ \\
\hline B1P1K6B & C5600 & 88.8 & $(1.53 \mathrm{E}+03)$ & $5.18 \mathrm{E}+00$ & $(4.99 \mathrm{E}+01)$ & $1.08 \mathrm{E}+03$ \\
\hline B1P1K6A & C5600 & 89.3 & $(4.70 \mathrm{E}+02)$ & $5.47 \mathrm{E}+00$ & $(3.10 \mathrm{E}+01)$ & $6.51 \mathrm{E}+02$ \\
\hline B1P3F9B & C5604 & 50.8 & $(2.75 \mathrm{E}+02)$ & $1.70 \mathrm{E}+01$ & $(1.11 \mathrm{E}+01)$ & $6.40 \mathrm{E}+02$ \\
\hline B1P3F9A & C5604 & 51.3 & $(2.05 \mathrm{E}+02)$ & $1.01 \mathrm{E}+01$ & $(5.39 \mathrm{E}+00)$ & $4.52 \mathrm{E}+02$ \\
\hline B1P3H0B & C5602 & 51.8 & $<2.25 \mathrm{E}+03$ & $1.94 \mathrm{E}+04$ & $(1.83 \mathrm{E}+01)$ & $6.99 \mathrm{E}+02$ \\
\hline B1P3H0A & C5602 & 52.3 & $<6.12 \mathrm{E}+03$ & $4.82 E+04$ & $(3.73 \mathrm{E}+01)$ & $1.28 \mathrm{E}+03$ \\
\hline B1P3H1B & C5602 & 67.8 & $(4.45 \mathrm{E}+02)$ & $4.52 \mathrm{E}+04$ & $(3.18 \mathrm{E}+01)$ & $6.57 \mathrm{E}+02$ \\
\hline B1P3H1A & C5602 & 68.3 & $(2.82 \mathrm{E}+02)$ & $5.36 \mathrm{E}+04$ & $(1.09 \mathrm{E}+01)$ & $5.04 \mathrm{E}+02$ \\
\hline B1P3H2B & C5602 & 82.8 & $(1.28 \mathrm{E}+03)$ & $2.65 E+04$ & $(9.78 \mathrm{E}+00)$ & $6.95 \mathrm{E}+02$ \\
\hline B1P3H2A & C5602 & 83.3 & $(2.30 \mathrm{E}+03)$ & $1.89 \mathrm{E}+04$ & $(1.50 \mathrm{E}+01)$ & $7.91 \mathrm{E}+02$ \\
\hline B1PBB0B & C5602 & 91.8 & $2.47 E+03$ & $4.57 \mathrm{E}+00$ & $(8.38 \mathrm{E}+00)$ & $8.53 \mathrm{E}+02$ \\
\hline B1PBB0A & C5602 & 92.3 & $(2.49 \mathrm{E}+03)$ & $2.98 \mathrm{E}+00$ & $(1.11 \mathrm{E}+01)$ & $8.75 \mathrm{E}+02$ \\
\hline B1PBB1B & C5606 & 51.8 & $(5.83 \mathrm{E}+01)$ & $3.07 \mathrm{E}+01$ & $(6.36 \mathrm{E}+00)$ & $1.19 \mathrm{E}+02$ \\
\hline B1PBB1A & C5606 & 52.3 & $(5.28 \mathrm{E}+01)$ & $1.25 \mathrm{E}+02$ & $(7.33 \mathrm{E}+00)$ & $2.24 \mathrm{E}+02$ \\
\hline B1PBB1A DUP & C5606 & 52.3 & $(1.05 \mathrm{E}+02)$ & $1.30 \mathrm{E}+02$ & $(5.98 \mathrm{E}+00)$ & $2.69 \mathrm{E}+02$ \\
\hline B1PK51B & C5608 & 64.3 & $9.08 E+02$ & $1.75 \mathrm{E}+01$ & $3.63 \mathrm{E}+02$ & $1.13 \mathrm{E}+02$ \\
\hline B1PK51A & C5608 & 64.8 & $7.43 E+02$ & $3.65 \mathrm{E}+01$ & $7.62 \mathrm{E}+01$ & $1.91 \mathrm{E}+03$ \\
\hline B1PK52B & C5608 & 85.8 & $2.50 \mathrm{E}+05$ & $4.81 \mathrm{E}+00$ & $3.08 E+04$ & $3.51 \mathrm{E}+02$ \\
\hline B1PK52A & C5608 & 86.3 & $2.42 E+05$ & $1.58 \mathrm{E}+01$ & $3.02 E+04$ & $4.08 \mathrm{E}+02$ \\
\hline B1PK53B & C5608 & 97.8 & $1.28 \mathrm{E}+05$ & $1.04 \mathrm{E}+03$ & $2.65 E+04$ & $1.65 \mathrm{E}+03$ \\
\hline B1PK53A & C5608 & 98.3 & $2.06 E+05$ & $1.24 \mathrm{E}+03$ & $3.64 E+04$ & $2.60 \mathrm{E}+03$ \\
\hline
\end{tabular}

\subsubsection{M Nitric Acid-Extractable Amounts of Selected Elements in the U Tank Farm Direct Push Sediments}

The same cores and grab samples that were characterized for water-leachable constituents were also characterized to determine the concentrations of constituents that could be extracted with hot $8 \mathrm{M}$ nitric acid. A comparison between the quantities that were acid-extractable with those that are water-extractable typically indicates the relative mobility of a given constituent and can be used to differentiate anthropogenic from naturally occurring constituents. The acid extractable concentrations are shown in Tables 4.28 through 4.30. For several of the constituents, there were no significantly elevated acid-extractable values in the U Tank Farm direct push sediments. However, sodium, chromium, technetium-99, and uranium-238 were all significantly elevated in at least some of the U Farm direct push samples. 
Elevated acid-extractable sodium was found in all of the U Farm direct push samples from push hole C5608 analyzed. Acid-extractable sodium concentrations ranged from 910 to $2520 \mu \mathrm{g} / \mathrm{g}$. These values compare to a range of 246 to $661 \mu \mathrm{g} / \mathrm{g}$ measured in the background borehole. The source of the contamination must be tank-related, and cation exchange is the likely mechanism that has caused its sequestration in the vadose zone. Elevated acid-extractable chromium was found in the four deepest samples analyzed from push hole C5608. Acid-extractable chromium concentrations ranged from 31.6 to $55.9 \mu \mathrm{g} / \mathrm{g}$, vs. a peak concentration on $23.0 \mu \mathrm{g} / \mathrm{g}$ in the background borehole. Acid-extractable technetium-99 was found in samples from push holes C5600, C5602, and C5608. Acid-extractable technetium-99 activities ranged from a low of $10.6 \mathrm{pCi} / \mathrm{g}$ in sample B1PK6B (from push hole C5600) to a high of $56.7 \mathrm{pCi} / \mathrm{g}$ in sample B1PK53B (from push hole C5608). This phenomenon, in which larger amounts of technetium-99 are found in the acid extracts than in the water extracts, has occurred at many other sampling campaigns and could be a result of sequestration of technetium-99 by iron oxides present in the sediments (Zachara et al. 2007). The final elevated acid-extractable constituent was uranium-238. Slightly elevated concentrations of acid-extractable uranium-238 $(1.35 \mu \mathrm{g} / \mathrm{g})$ were found in samples from push hole C5606, but significantly elevated concentrations (as much as $731 \mu \mathrm{g} / \mathrm{g}$ ) were measured in extracts of sediments from push hole C5602 (Figure 4.5). Push hole C5602 was emplaced between tank 241-U-104 and 241-U-105, and as mentioned previously, tank 241-U-104 was estimated to have released more than $5100 \mathrm{~kg}$ of uranium to the vadose zone. In addition, previous logging of drywells in the area has identified significant uranium contamination in the area of tank 241-U-104; therefore, the observation of more than $700 \mu \mathrm{g} / \mathrm{g}$ uranium in sediment from push hole C5602 was not unexpected.

Table 4.28. Acid-Extractable Cations in the U Tank Farm Direct Push Samples ( $\mu \mathrm{g} / \mathrm{g}$ dry sediment)

\begin{tabular}{||l|c|c|c|c|c|c||}
\hline $\begin{array}{c}\text { Sample } \\
\text { ID }\end{array}$ & $\begin{array}{c}\text { Probe Hole } \\
\text { ID }\end{array}$ & $\begin{array}{c}\text { Mid-Depth } \\
\mathrm{ft} \text { bgs }\end{array}$ & $\begin{array}{c}\text { Calcium } \\
\mu \mathrm{g} / \mathrm{g}\end{array}$ & $\begin{array}{c}\text { Potassium } \\
\mu \mathrm{g} / \mathrm{g}\end{array}$ & $\begin{array}{c}\text { Magnesium } \\
\mu \mathrm{g} / \mathrm{g}\end{array}$ & $\begin{array}{c}\text { Sodium } \\
\mu \mathrm{g} / \mathrm{g}\end{array}$ \\
\hline \hline B1NDW3B & C5590 & 96.3 & $9.40 \mathrm{E}+03$ & $1.52 \mathrm{E}+03$ & $5.40 \mathrm{E}+03$ & $1.65 \mathrm{E}+02$ \\
\hline B1NDW3A & C5590 & 96.8 & $7.03 \mathrm{E}+03$ & $1.31 \mathrm{E}+03$ & $4.38 \mathrm{E}+03$ & $1.28 \mathrm{E}+02$ \\
\hline B1NDW3A DUP & C5590 & 96.8 & $1.23 \mathrm{E}+04$ & $2.51 \mathrm{E}+03$ & $7.73 \mathrm{E}+03$ & $2.86 \mathrm{E}+02$ \\
\hline B1NHV0B & C5592 & 62.3 & $8.86 \mathrm{E}+03$ & $1.83 \mathrm{E}+03$ & $5.86 \mathrm{E}+03$ & $1.96 \mathrm{E}+02$ \\
\hline B1NHV0A & C5592 & 62.8 & $8.50 \mathrm{E}+03$ & $1.43 \mathrm{E}+03$ & $5.06 \mathrm{E}+03$ & $1.59 \mathrm{E}+02$ \\
\hline B1NTC6B & C5598 & 50.3 & $1.02 \mathrm{E}+04$ & $1.58 \mathrm{E}+03$ & $5.45 \mathrm{E}+03$ & $1.81 \mathrm{E}+02$ \\
\hline B1NTC6A & C5598 & 50.8 & $1.22 \mathrm{E}+04$ & $1.72 \mathrm{E}+03$ & $6.12 \mathrm{E}+03$ & $2.29 \mathrm{E}+02$ \\
\hline B1NTC7B & C5598 & 59.8 & $1.05 \mathrm{E}+04$ & $1.88 \mathrm{E}+03$ & $5.92 \mathrm{E}+03$ & $1.70 \mathrm{E}+02$ \\
\hline B1NTC7A & C5598 & 60.3 & $9.28 \mathrm{E}+03$ & $1.29 \mathrm{E}+03$ & $5.02 \mathrm{E}+03$ & $1.49 \mathrm{E}+02$ \\
\hline B1NTC8B & C5598 & 82.3 & $1.04 \mathrm{E}+04$ & $2.38 \mathrm{E}+03$ & $6.85 \mathrm{E}+03$ & $2.63 \mathrm{E}+02$ \\
\hline B1NTC8B DUP & C5598 & 82.3 & $1.01 \mathrm{E}+04$ & $2.30 \mathrm{E}+03$ & $6.83 \mathrm{E}+03$ & $2.36 \mathrm{E}+02$ \\
\hline B1NTC8A & C5598 & 82.8 & $9.03 \mathrm{E}+03$ & $1.80 \mathrm{E}+03$ & $5.72 \mathrm{E}+03$ & $1.51 \mathrm{E}+02$ \\
\hline B1NTC9B & C5596 & 50.8 & $1.02 \mathrm{E}+04$ & $1.40 \mathrm{E}+03$ & $5.40 \mathrm{E}+03$ & $2.31 \mathrm{E}+02$ \\
\hline B1NTC9A & C5596 & 51.3 & $1.03 \mathrm{E}+04$ & $1.43 \mathrm{E}+03$ & $5.46 \mathrm{E}+03$ & $1.82 \mathrm{E}+02$ \\
\hline B1NTD0B & C5596 & 61.3 & $9.33 \mathrm{E}+03$ & $1.35 \mathrm{E}+03$ & $5.15 \mathrm{E}+03$ & $1.34 \mathrm{E}+02$ \\
\hline B1NTD0A & C5596 & 61.8 & $9.15 \mathrm{E}+03$ & $1.54 \mathrm{E}+03$ & $5.29 \mathrm{E}+03$ & $1.58 \mathrm{E}+02$ \\
\hline B1NTD1B & C5596 & 77.8 & $9.53 \mathrm{E}+03$ & $1.71 \mathrm{E}+03$ & $5.66 \mathrm{E}+03$ & $2.14 \mathrm{E}+02$ \\
\hline B1NTD1B DUP & C5596 & 77.8 & $9.07 \mathrm{E}+03$ & $1.57 \mathrm{E}+03$ & $5.26 \mathrm{E}+03$ & $1.42 \mathrm{E}+02$ \\
\hline B1NTD1A & C5596 & 78.3 & $9.47 \mathrm{E}+03$ & $1.91 \mathrm{E}+03$ & $5.86 \mathrm{E}+03$ & $2.37 \mathrm{E}+02$ \\
\hline B1NTD2B & C5596 & 82.8 & $9.04 \mathrm{E}+03$ & $1.17 \mathrm{E}+03$ & $4.78 \mathrm{E}+03$ & $1.56 \mathrm{E}+02$ \\
\hline B1NTD2A & C5596 & 83.3 & $9.24 \mathrm{E}+03$ & $1.22 \mathrm{E}+03$ & $4.66 \mathrm{E}+03$ & $1.56 \mathrm{E}+02$ \\
\hline B1NTD3B & C5600 & 50.3 & $1.08 \mathrm{E}+04$ & $1.72 \mathrm{E}+03$ & $6.04 \mathrm{E}+03$ & $1.85 \mathrm{E}+02$ \\
\hline
\end{tabular}




\begin{tabular}{|c|c|c|c|c|c|c|}
\hline $\begin{array}{l}\text { Sample } \\
\text { ID }\end{array}$ & $\begin{array}{c}\text { Probe Hole } \\
\text { ID }\end{array}$ & $\begin{array}{l}\text { Mid-Depth } \\
\mathrm{ft} \text { bgs }\end{array}$ & $\begin{array}{c}\text { Calcium } \\
\mu \mathrm{g} / \mathrm{g}\end{array}$ & $\begin{array}{c}\text { Potassium } \\
\mu \mathrm{g} / \mathrm{g}\end{array}$ & $\begin{array}{c}\text { Magnesium } \\
\mu \mathrm{g} / \mathrm{g}\end{array}$ & $\begin{array}{c}\text { Sodium } \\
\mu \mathrm{g} / \mathrm{g}\end{array}$ \\
\hline B1NTD3A & C5600 & 50.8 & $1.10 \mathrm{E}+04$ & $1.77 \mathrm{E}+03$ & $5.79 \mathrm{E}+03$ & $1.91 \mathrm{E}+02$ \\
\hline B1NTD4B & C5600 & 60.3 & $9.20 \mathrm{E}+03$ & $1.55 \mathrm{E}+03$ & $5.46 \mathrm{E}+03$ & $1.68 \mathrm{E}+02$ \\
\hline B1NTD4A & C5600 & 60.8 & $1.02 \mathrm{E}+04$ & $1.52 \mathrm{E}+03$ & $5.64 \mathrm{E}+03$ & $1.66 \mathrm{E}+02$ \\
\hline B1NTD5B & C5600 & 82.3 & $1.03 \mathrm{E}+04$ & $2.33 \mathrm{E}+03$ & $6.90 \mathrm{E}+03$ & $1.93 \mathrm{E}+02$ \\
\hline B1NTD5A & C5600 & 82.8 & $8.56 \mathrm{E}+03$ & $1.57 \mathrm{E}+03$ & $5.15 \mathrm{E}+03$ & $1.41 \mathrm{E}+02$ \\
\hline B1P1K6B & C5600 & 88.8 & $7.87 \mathrm{E}+03$ & $1.42 \mathrm{E}+03$ & $4.99 \mathrm{E}+03$ & $1.42 \mathrm{E}+02$ \\
\hline B1P1K6A & C5600 & 89.3 & $8.78 \mathrm{E}+03$ & $1.84 \mathrm{E}+03$ & $5.34 \mathrm{E}+03$ & $1.41 \mathrm{E}+02$ \\
\hline B1P3F9B & C5604 & 50.8 & $1.07 \mathrm{E}+04$ & $1.53 \mathrm{E}+03$ & $5.64 \mathrm{E}+03$ & $1.70 \mathrm{E}+02$ \\
\hline B1P3F9A & C5604 & 51.3 & $9.23 \mathrm{E}+03$ & $1.16 \mathrm{E}+03$ & $5.34 \mathrm{E}+03$ & $1.47 \mathrm{E}+02$ \\
\hline B1P3H0B & C5602 & 51.8 & $8.87 \mathrm{E}+03$ & $1.06 \mathrm{E}+03$ & $4.11 \mathrm{E}+03$ & $1.05 \mathrm{E}+02$ \\
\hline B1P3H0A & C5602 & 52.3 & $7.94 \mathrm{E}+03$ & $9.30 \mathrm{E}+02$ & $4.48 \mathrm{E}+03$ & $1.27 \mathrm{E}+02$ \\
\hline B1P3H1B & C5602 & 67.8 & $8.83 \mathrm{E}+03$ & $1.37 \mathrm{E}+03$ & $5.13 \mathrm{E}+03$ & $1.73 \mathrm{E}+02$ \\
\hline B1P3H1A & C5602 & 68.3 & $8.25 \mathrm{E}+03$ & $1.56 \mathrm{E}+03$ & $5.31 \mathrm{E}+03$ & $1.62 \mathrm{E}+02$ \\
\hline B1P3H2B & C5602 & 82.8 & $9.96 \mathrm{E}+03$ & $1.67 \mathrm{E}+03$ & $5.77 \mathrm{E}+03$ & $1.77 \mathrm{E}+02$ \\
\hline B1P3H2A & C5602 & 83.3 & $1.00 \mathrm{E}+04$ & $1.38 \mathrm{E}+03$ & $5.64 \mathrm{E}+03$ & $1.77 \mathrm{E}+02$ \\
\hline B1PBB0B & C5602 & 91.8 & $8.58 \mathrm{E}+03$ & $1.51 \mathrm{E}+03$ & $5.12 \mathrm{E}+03$ & $1.74 \mathrm{E}+02$ \\
\hline B1PBB0A & C5602 & 92.3 & $8.27 \mathrm{E}+03$ & $1.30 \mathrm{E}+03$ & $4.37 \mathrm{E}+03$ & $1.06 \mathrm{E}+02$ \\
\hline B1PBB1B & C5606 & 51.8 & $1.00 \mathrm{E}+04$ & $1.65 \mathrm{E}+03$ & $5.23 \mathrm{E}+03$ & $1.58 \mathrm{E}+02$ \\
\hline B1PBB1A & C5606 & 52.3 & $1.20 \mathrm{E}+04$ & $1.98 \mathrm{E}+03$ & $6.22 \mathrm{E}+03$ & $1.98 \mathrm{E}+02$ \\
\hline B1PBB1A DUP & C5606 & 52.3 & $1.24 \mathrm{E}+04$ & $2.02 \mathrm{E}+03$ & $6.40 \mathrm{E}+03$ & $2.03 \mathrm{E}+02$ \\
\hline B1PK51B & C5608 & 64.3 & $9.73 \mathrm{E}+03$ & $1.94 \mathrm{E}+03$ & $5.78 \mathrm{E}+03$ & $1.26 \mathrm{E}+03$ \\
\hline B1PK51A & C5608 & 64.8 & $8.42 \mathrm{E}+03$ & $1.37 \mathrm{E}+03$ & $4.75 \mathrm{E}+03$ & $9.10 \mathrm{E}+02$ \\
\hline B1PK52B & C5608 & 85.8 & $9.29 \mathrm{E}+03$ & $2.48 \mathrm{E}+03$ & $6.63 \mathrm{E}+03$ & $3.07 E+03$ \\
\hline B1PK52A & C5608 & 86.3 & $8.48 \mathrm{E}+03$ & $1.92 \mathrm{E}+03$ & $5.54 \mathrm{E}+03$ & $2.52 \mathrm{E}+03$ \\
\hline B1PK53B & C5608 & 97.8 & $8.28 \mathrm{E}+03$ & $2.10 \mathrm{E}+03$ & $5.74 \mathrm{E}+03$ & $1.66 \mathrm{E}+03$ \\
\hline B1PK53A & C5608 & 98.3 & $7.69 \mathrm{E}+03$ & $1.62 \mathrm{E}+03$ & $4.95 \mathrm{E}+03$ & $1.10 \mathrm{E}+03$ \\
\hline
\end{tabular}

Table 4.29. Acid-Leachable Cations in the U Tank Farm Core and Grab Samples ( $\mu \mathrm{g} / \mathrm{g}$ dry sediment)

\begin{tabular}{||l|c|c|c|c|c|c||}
\hline $\begin{array}{c}\text { Sample } \\
\text { ID }\end{array}$ & $\begin{array}{c}\text { Probe Hole } \\
\text { ID }\end{array}$ & $\begin{array}{c}\text { Mid-Depth } \\
\text { ft bgs }\end{array}$ & $\begin{array}{c}\text { Aluminum } \\
\mu \mathrm{g} / \mathrm{g}\end{array}$ & $\begin{array}{c}\text { Iron } \\
\mu \mathrm{g} / \mathrm{g}\end{array}$ & $\begin{array}{c}\text { Phosphorus } \\
(\text { as Phosphate) } \\
\mu \mathrm{g} / \mathrm{g}\end{array}$ & $\begin{array}{c}\text { Sulfur } \\
\text { (as Sulfate) } \\
\mu \mathrm{g} / \mathrm{g}\end{array}$ \\
\hline \hline B1NDW3B & C5590 & 96.3 & $7.91 \mathrm{E}+03$ & $1.49 \mathrm{E}+04$ & $2.09 \mathrm{E}+03$ & $<3.63 \mathrm{E}+02$ \\
\hline B1NDW3A & C5590 & 96.8 & $6.65 \mathrm{E}+03$ & $1.20 \mathrm{E}+04$ & $1.54 \mathrm{E}+03$ & $<3.24 \mathrm{E}+02$ \\
\hline B1NDW3A DUP & C5590 & 96.8 & $1.34 \mathrm{E}+04$ & $2.21 \mathrm{E}+04$ & $2.62 \mathrm{E}+03$ & $<3.97 \mathrm{E}+02$ \\
\hline B1NHV0B & C5592 & 62.3 & $8.70 \mathrm{E}+03$ & $1.56 \mathrm{E}+04$ & $1.69 \mathrm{E}+03$ & $<3.58 \mathrm{E}+02$ \\
\hline B1NHV0A & C5592 & 62.8 & $6.61 \mathrm{E}+03$ & $1.38 \mathrm{E}+04$ & $1.88 \mathrm{E}+03$ & $<3.22 \mathrm{E}+02$ \\
\hline B1NTC6B & C5598 & 50.3 & $7.35 \mathrm{E}+03$ & $1.40 \mathrm{E}+04$ & $1.57 \mathrm{E}+03$ & $<3.21 \mathrm{E}+02$ \\
\hline B1NTC6A & C5598 & 50.8 & $9.03 \mathrm{E}+03$ & $1.58 \mathrm{E}+04$ & $1.95 \mathrm{E}+03$ & $<3.14 \mathrm{E}+02$ \\
\hline B1NTC7B & C5598 & 59.8 & $8.60 \mathrm{E}+03$ & $1.51 \mathrm{E}+04$ & $1.40 \mathrm{E}+03$ & $<3.60 \mathrm{E}+02$ \\
\hline B1NTC7A & C5598 & 60.3 & $6.47 \mathrm{E}+03$ & $1.26 \mathrm{E}+04$ & $1.56 \mathrm{E}+03$ & $<3.20 \mathrm{E}+02$ \\
\hline B1NTC8B & C5598 & 82.3 & $1.22 \mathrm{E}+04$ & $1.96 \mathrm{E}+04$ & $2.08 \mathrm{E}+03$ & $<3.17 \mathrm{E}+02$ \\
\hline B1NTC8B DUP & C5598 & 82.3 & $1.17 \mathrm{E}+04$ & $1.91 \mathrm{E}+04$ & $2.04 \mathrm{E}+03$ & $<3.15 \mathrm{E}+02$ \\
\hline B1NTC8A & C5598 & 82.8 & $7.68 \mathrm{E}+03$ & $1.45 \mathrm{E}+04$ & $1.70 \mathrm{E}+03$ & $<3.27 \mathrm{E}+02$ \\
\hline
\end{tabular}




\begin{tabular}{|c|c|c|c|c|c|c|}
\hline $\begin{array}{c}\text { Sample } \\
\text { ID }\end{array}$ & $\begin{array}{c}\text { Probe Hole } \\
\text { ID }\end{array}$ & $\begin{array}{c}\text { Mid-Depth } \\
\text { ft bgs }\end{array}$ & $\begin{array}{c}\text { Aluminum } \\
\mu \mathrm{g} / \mathrm{g}\end{array}$ & $\begin{array}{l}\text { Iron } \\
\mu \mathrm{g} / \mathrm{g}\end{array}$ & $\begin{array}{c}\text { Phosphorus } \\
\text { (as Phosphate) } \\
\mu \mathrm{g} / \mathrm{g}\end{array}$ & $\begin{array}{c}\text { Sulfur } \\
\text { (as Sulfate) } \\
\mu \mathrm{g} / \mathrm{g} \\
\end{array}$ \\
\hline B1NTC9B & "C5596 & 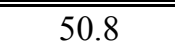 & $=7.22 \mathrm{E}+03$ & $1.49 \mathrm{E}+04$ & $1.80 \mathrm{E}+03$ & 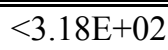 \\
\hline B1NTC9A & C5596 & 51.3 & $7.35 \mathrm{E}+03$ & $1.45 \mathrm{E}+04$ & $1.44 \mathrm{E}+03$ & $<3.34 \mathrm{E}+02$ \\
\hline B1NTD0B & C5596 & 61.3 & $6.35 \mathrm{E}+03$ & $1.22 \mathrm{E}+04$ & $2.03 \mathrm{E}+03$ & $<3.62 \mathrm{E}+02$ \\
\hline B1NTD0A & C5596 & 61.8 & $6.99 \mathrm{E}+03$ & $1.34 \mathrm{E}+04$ & $1.76 \mathrm{E}+03$ & $<3.14 \mathrm{E}+02$ \\
\hline B1NTD1B & C5596 & 77.8 & $9.06 \mathrm{E}+03$ & $1.61 \mathrm{E}+04$ & $1.82 \mathrm{E}+03$ & $<3.55 \mathrm{E}+02$ \\
\hline B1NTD1B DUP & C5596 & 77.8 & $6.99 \mathrm{E}+03$ & $1.35 \mathrm{E}+04$ & $1.90 \mathrm{E}+03$ & $<3.20 \mathrm{E}+02$ \\
\hline B1NTD1A & C5596 & 78.3 & $9.63 \mathrm{E}+03$ & $1.66 \mathrm{E}+04$ & $1.77 \mathrm{E}+03$ & $<3.12 \mathrm{E}+02$ \\
\hline B1NTD2B & C5596 & 82.8 & $6.26 \mathrm{E}+03$ & $1.29 \mathrm{E}+04$ & $1.49 \mathrm{E}+03$ & $<3.04 \mathrm{E}+02$ \\
\hline B1NTD2A & C5596 & 83.3 & $6.21 \mathrm{E}+03$ & $1.29 \mathrm{E}+04$ & $1.41 \mathrm{E}+03$ & $<2.92 \mathrm{E}+02$ \\
\hline B1NTD3B & $\mathrm{C} 5600$ & 50.3 & $8.77 \mathrm{E}+03$ & $1.59 \mathrm{E}+04$ & $1.81 \mathrm{E}+03$ & $<3.45 \mathrm{E}+02$ \\
\hline B1NTD3A & C5600 & 50.8 & $8.47 \mathrm{E}+03$ & $1.54 \mathrm{E}+04$ & $1.80 \mathrm{E}+03$ & $<3.39 \mathrm{E}+02$ \\
\hline B1NTD4B & $\mathrm{C} 5600$ & 60.3 & $7.60 \mathrm{E}+03$ & $1.49 \mathrm{E}+04$ & $1.53 \mathrm{E}+03$ & $<3.19 \mathrm{E}+02$ \\
\hline B1NTD4A & $\mathrm{C} 5600$ & 60.8 & $7.73 \mathrm{E}+03$ & $1.50 \mathrm{E}+04$ & $1.66 \mathrm{E}+03$ & $<3.29 \mathrm{E}+02$ \\
\hline B1NTD5B & C5600 & 82.3 & $1.04 \mathrm{E}+04$ & $1.88 \mathrm{E}+04$ & $2.08 \mathrm{E}+03$ & $<3.80 \mathrm{E}+02$ \\
\hline B1NTD5A & C5600 & 82.8 & $6.80 \mathrm{E}+03$ & $1.36 \mathrm{E}+04$ & $1.49 \mathrm{E}+03$ & $<3.12 \mathrm{E}+02$ \\
\hline B1P1K6B & $\mathrm{C} 5600$ & 88.8 & $6.70 \mathrm{E}+03$ & $1.33 \mathrm{E}+04$ & $1.58 \mathrm{E}+03$ & $<3.11 \mathrm{E}+02$ \\
\hline B1P1K6A & C5600 & 89.3 & $7.46 \mathrm{E}+03$ & $1.40 \mathrm{E}+04$ & $2.05 \mathrm{E}+03$ & $<3.31 \mathrm{E}+02$ \\
\hline B1P3F9B & C5604 & 50.8 & $7.03 \mathrm{E}+03$ & $1.40 \mathrm{E}+04$ & $1.83 \mathrm{E}+03$ & $<3.72 \mathrm{E}+02$ \\
\hline B1P3F9A & C5604 & 51.3 & $6.55 \mathrm{E}+03$ & $1.36 \mathrm{E}+04$ & $1.69 \mathrm{E}+03$ & $<3.36 \mathrm{E}+02$ \\
\hline B1P3H0B & C5602 & 51.8 & $4.63 \mathrm{E}+03$ & $9.78 \mathrm{E}+03$ & $1.56 \mathrm{E}+03$ & $<3.55 \mathrm{E}+02$ \\
\hline B1P3H0A & C5602 & 52.3 & $5.30 \mathrm{E}+03$ & $1.16 \mathrm{E}+04$ & $1.36 \mathrm{E}+03$ & $<3.18 \mathrm{E}+02$ \\
\hline B1P3H1B & $\mathrm{C} 5602$ & 67.8 & $7.32 \mathrm{E}+03$ & $1.39 \mathrm{E}+04$ & $1.38 \mathrm{E}+03$ & $<3.50 \mathrm{E}+02$ \\
\hline B1P3H1A & C5602 & 68.3 & $7.60 \mathrm{E}+03$ & $1.39 \mathrm{E}+04$ & $1.54 \mathrm{E}+03$ & $<3.88 \mathrm{E}+02$ \\
\hline B1P3H2B & C5602 & 82.8 & $8.18 \mathrm{E}+03$ & $1.56 \mathrm{E}+04$ & $1.83 \mathrm{E}+03$ & $<3.46 \mathrm{E}+02$ \\
\hline B1P3H2A & C5602 & 83.3 & $7.70 \mathrm{E}+03$ & $1.58 \mathrm{E}+04$ & $1.73 \mathrm{E}+03$ & $<3.28 \mathrm{E}+02$ \\
\hline B1PBB0B & C5602 & 91.8 & $7.55 \mathrm{E}+03$ & $1.49 \mathrm{E}+04$ & $1.68 \mathrm{E}+03$ & $<3.85 \mathrm{E}+02$ \\
\hline B1PBB0A & C5602 & 92.3 & $5.40 \mathrm{E}+03$ & $1.10 \mathrm{E}+04$ & $1.79 \mathrm{E}+03$ & $<3.70 \mathrm{E}+02$ \\
\hline B1PBB1B & C5606 & 51.8 & $6.83 \mathrm{E}+03$ & $1.33 \mathrm{E}+04$ & $1.67 \mathrm{E}+03$ & $<1.64 \mathrm{E}+03$ \\
\hline B1PBB1A & C5606 & 52.3 & $8.73 \mathrm{E}+03$ & $1.63 \mathrm{E}+04$ & $1.95 \mathrm{E}+03$ & $<1.64 \mathrm{E}+03$ \\
\hline B1PBB1A DUP & C5606 & 52.3 & $8.85 \mathrm{E}+03$ & $1.67 \mathrm{E}+04$ & $2.04 \mathrm{E}+03$ & $<1.65 \mathrm{E}+03$ \\
\hline B1PK51B & C5608 & 64.3 & $8.32 \mathrm{E}+03$ & $1.55 \mathrm{E}+04$ & $1.86 \mathrm{E}+03$ & $<1.61 \mathrm{E}+03$ \\
\hline B1PK51A & C5608 & 64.8 & $6.39 \mathrm{E}+03$ & $1.28 \mathrm{E}+04$ & $1.42 \mathrm{E}+03$ & $<1.56 \mathrm{E}+03$ \\
\hline B1PK52B & C5608 & 85.8 & $1.08 \mathrm{E}+04$ & $1.92 \mathrm{E}+04$ & $2.00 \mathrm{E}+03$ & $<1.66 \mathrm{E}+03$ \\
\hline B1PK52A & C5608 & 86.3 & $8.10 \mathrm{E}+03$ & $1.50 \mathrm{E}+04$ & $2.17 \mathrm{E}+03$ & $<1.52 \mathrm{E}+03$ \\
\hline B1PK53B & C5608 & 97.8 & $8.33 \mathrm{E}+03$ & $1.54 \mathrm{E}+04$ & $1.83 \mathrm{E}+03$ & $<1.70 \mathrm{E}+03$ \\
\hline B1PK53A & C5608 & 98.3 & $6.58 \mathrm{E}+03$ & $1.28 \mathrm{E}+04$ & $1.54 \mathrm{E}+03$ & $<1.55 \mathrm{E}+03$ \\
\hline
\end{tabular}


Table 4.30. Acid-Extractable Mobile Metals in the U Tank Farm Direct Push Samples $(\mu \mathrm{g} / \mathrm{g}$ dry sediment)

\begin{tabular}{|c|c|c|c|c|c|c|c|}
\hline $\begin{array}{l}\text { Sample } \\
\text { ID }\end{array}$ & $\begin{array}{c}\text { Probe } \\
\text { Hole } \\
\text { ID }\end{array}$ & $\begin{array}{l}\text { Mid- } \\
\text { Depth } \\
\mathrm{ft} \text { bgs }\end{array}$ & $\begin{array}{c}\text { Technetium- } \\
99 \\
\text { pCi/g }\end{array}$ & $\begin{array}{c}\text { Uranium- } \\
238 \\
\mu \mathrm{g} / \mathrm{g}\end{array}$ & $\begin{array}{c}\text { Molybdenum } \\
\mu \mathrm{g} / \mathrm{g}\end{array}$ & $\begin{array}{c}\text { Chromium } \\
\mu \mathrm{g} / \mathrm{g}\end{array}$ & $\begin{array}{l}\text { Lead } \\
\mu \mathrm{g} / \mathrm{g}\end{array}$ \\
\hline B1NDW3B & בC5590 & 96.3 & $\begin{array}{c}<2.05 \mathrm{E}+00 \\
\end{array}$ & 5.03E-01 & $3.80 \mathrm{E}-01$ & $=1.36 \mathrm{E}+01$ & $4.46 \mathrm{E}+00$ \\
\hline B1NDW3A & C5590 & 96.8 & $<9.16 \mathrm{E}-01$ & $4.52 \mathrm{E}-01$ & $(2.65 \mathrm{E}-01)$ & $1.00 \mathrm{E}+01$ & $3.82 \mathrm{E}+00$ \\
\hline B1NDW3A DUP & C5590 & 96.8 & $<1.12 \mathrm{E}+01$ & $7.82 \mathrm{E}-01$ & $5.19 \mathrm{E}-01$ & $1.64 \mathrm{E}+01$ & $6.07 \mathrm{E}+00$ \\
\hline B1NHV0B & C5592 & 62.3 & $<2.02 \mathrm{E}+00$ & $5.65 \mathrm{E}-01$ & $(2.71 \mathrm{E}-01)$ & $1.32 \mathrm{E}+01$ & $4.94 \mathrm{E}+00$ \\
\hline B1NHV0A & C5592 & 62.8 & $<1.82 \mathrm{E}+00$ & $4.10 \mathrm{E}-01$ & $(2.12 \mathrm{E}-01)$ & $1.01 \mathrm{E}+01$ & $3.89 \mathrm{E}+00$ \\
\hline B1NTC6B & C5598 & 50.3 & $<2.72 \mathrm{E}+00$ & $4.48 \mathrm{E}-01$ & $(2.63 \mathrm{E}-01)$ & $1.24 \mathrm{E}+01$ & $5.54 \mathrm{E}+00$ \\
\hline B1NTC6A & C5598 & 50.8 & $<8.86 \mathrm{E}+00$ & $5.83 \mathrm{E}-01$ & $3.16 \mathrm{E}-01$ & $1.12 \mathrm{E}+01$ & $5.43 \mathrm{E}+00$ \\
\hline B1NTC7B & C5598 & 59.8 & $<8.14 \mathrm{E}+00$ & 4.89E-01 & $(2.47 \mathrm{E}-01)$ & $1.16 \mathrm{E}+01$ & $5.11 \mathrm{E}+00$ \\
\hline B1NTC7A & C5598 & 60.3 & $<8.13 \mathrm{E}+00$ & $4.64 \mathrm{E}-01$ & $(2.35 \mathrm{E}-01)$ & $8.74 \mathrm{E}+00$ & $4.88 \mathrm{E}+00$ \\
\hline B1NTC8B & C5598 & 82.3 & $<3.59 \mathrm{E}+00$ & $5.77 \mathrm{E}-01$ & $3.34 \mathrm{E}-01$ & $1.38 \mathrm{E}+01$ & $5.16 \mathrm{E}+00$ \\
\hline B1NTC8B DUP & C5598 & 82.3 & $<4.45 \mathrm{E}+00$ & $5.60 \mathrm{E}-01$ & $2.73 \mathrm{E}-01$ & $1.28 \mathrm{E}+01$ & $5.55 \mathrm{E}+00$ \\
\hline B1NTC8A & C5598 & 82.8 & $<9.25 \mathrm{E}-01$ & 4.97E-01 & $(2.34 \mathrm{E}-01)$ & $1.07 \mathrm{E}+01$ & $5.06 \mathrm{E}+00$ \\
\hline B1NTC9B & C5596 & 50.8 & $<3.60 \mathrm{E}+00$ & $5.22 \mathrm{E}-01$ & $2.87 \mathrm{E}-01$ & $1.06 \mathrm{E}+01$ & $5.13 \mathrm{E}+00$ \\
\hline B1NTC9A & C5596 & 51.3 & $<9.44 \mathrm{E}-01$ & $4.81 \mathrm{E}-01$ & $2.80 \mathrm{E}-01$ & $1.11 \mathrm{E}+01$ & $5.91 \mathrm{E}+00$ \\
\hline B1NTD0B & C5596 & 61.3 & $<2.05 \mathrm{E}+00$ & $5.01 \mathrm{E}-01$ & $(2.71 \mathrm{E}-01)$ & $1.64 \mathrm{E}+01$ & $4.44 \mathrm{E}+00$ \\
\hline B1NTD0A & C5596 & 61.8 & $<2.67 \mathrm{E}+00$ & $4.12 \mathrm{E}-01$ & 4.99E-01 & $1.32 \mathrm{E}+01$ & $4.14 \mathrm{E}+00$ \\
\hline B1NTD1B & C5596 & 77.8 & $<2.01 \mathrm{E}+00$ & $5.14 \mathrm{E}-01$ & $5.32 \mathrm{E}-01$ & $2.09 \mathrm{E}+01$ & $4.55 \mathrm{E}+00$ \\
\hline B1NTD1B DUP & C5596 & 77.8 & $<2.71 \mathrm{E}+00$ & 4.07E-01 & $4.17 \mathrm{E}-01$ & $1.89 \mathrm{E}+01$ & $4.33 \mathrm{E}+00$ \\
\hline B1NTD1A & C5596 & 78.3 & $<8.83 \mathrm{E}-01$ & $5.91 \mathrm{E}-01$ & 4.64E-01 & $1.66 \mathrm{E}+01$ & $4.84 \mathrm{E}+00$ \\
\hline B1NTD2B & C5596 & 82.8 & $<2.58 \mathrm{E}+00$ & 4.67E-01 & $3.40 \mathrm{E}-01$ & $1.45 \mathrm{E}+01$ & $3.67 \mathrm{E}+00$ \\
\hline B1NTD2A & C5596 & 83.3 & $<1.65 \mathrm{E}+00$ & $4.41 \mathrm{E}-01$ & $2.87 \mathrm{E}-01$ & $9.35 \mathrm{E}+00$ & $3.59 \mathrm{E}+00$ \\
\hline B1NTD3B & C5600 & 50.3 & $<9.76 \mathrm{E}-01$ & $5.61 \mathrm{E}-01$ & $(2.46 \mathrm{E}-01)$ & $1.02 \mathrm{E}+01$ & $5.22 \mathrm{E}+00$ \\
\hline B1NTD3A & $\mathrm{C} 5600$ & 50.8 & $<2.88 \mathrm{E}+00$ & 5.32E-01 & $3.19 \mathrm{E}-01$ & $1.10 \mathrm{E}+01$ & $5.43 \mathrm{E}+00$ \\
\hline B1NTD4B & $\mathrm{C} 5600$ & 60.3 & $<3.61 \mathrm{E}+00$ & $4.33 \mathrm{E}-01$ & $4.73 \mathrm{E}-01$ & $1.95 \mathrm{E}+01$ & $3.85 \mathrm{E}+00$ \\
\hline B1NTD4A & C5600 & 60.8 & $<9.31 \mathrm{E}+00$ & 4.69E-01 & $3.32 \mathrm{E}-01$ & $1.65 \mathrm{E}+01$ & $4.54 \mathrm{E}+00$ \\
\hline B1NTD5B & $\mathrm{C} 5600$ & 82.3 & $<2.15 \mathrm{E}+00$ & $5.41 \mathrm{E}-01$ & $4.96 \mathrm{E}-01$ & $1.99 \mathrm{E}+01$ & $5.98 \mathrm{E}+00$ \\
\hline B1NTD5A & $\mathrm{C} 5600$ & 82.8 & $<8.82 \mathrm{E}+00$ & $4.17 \mathrm{E}-01$ & $3.28 \mathrm{E}-01$ & $1.16 \mathrm{E}+01$ & $4.07 \mathrm{E}+00$ \\
\hline B1P1K6B & C5600 & 88.8 & $1.06 \mathrm{E}+01$ & $4.16 \mathrm{E}-01$ & 3.91E-01 & $1.42 \mathrm{E}+01$ & $4.00 \mathrm{E}+00$ \\
\hline B1P1K6A & $\mathrm{C} 5600$ & 89.3 & $<8.42 \mathrm{E}+00$ & 4.47E-01 & $3.06 \mathrm{E}-01$ & $1.40 \mathrm{E}+01$ & $4.57 \mathrm{E}+00$ \\
\hline B1P3F9B & C5604 & 50.8 & $<6.31 \mathrm{E}+00$ & $5.22 \mathrm{E}-01$ & $5.07 \mathrm{E}-01$ & $1.30 \mathrm{E}+01$ & $5.48 \mathrm{E}+00$ \\
\hline B1P3F9A & C5604 & 51.3 & $<4.76 \mathrm{E}+00$ & $4.91 \mathrm{E}-01$ & $(2.73 \mathrm{E}-01)$ & $9.25 \mathrm{E}+00$ & $4.76 \mathrm{E}+00$ \\
\hline B1P3H0B & $\mathrm{C} 5602$ & 51.8 & $1.30 \mathrm{E}+01$ & $7.31 \mathrm{E}+02$ & $(1.85 \mathrm{E}-01)$ & $9.42 \mathrm{E}+00$ & $4.13 \mathrm{E}+00$ \\
\hline B1P3H0A & C5602 & 52.3 & $<1.80 \mathrm{E}+00$ & $4.14 \mathrm{E}+02$ & $3.91 \mathrm{E}-01$ & $1.21 \mathrm{E}+01$ & $3.82 \mathrm{E}+00$ \\
\hline B1P3H1B & C5602 & 67.8 & $<9.88 \mathrm{E}+00$ & $1.52 \mathrm{E}+01$ & $3.76 \mathrm{E}-01$ & $1.78 \mathrm{E}+01$ & $3.63 \mathrm{E}+00$ \\
\hline B1P3H1A & C5602 & 68.3 & $<5.49 \mathrm{E}+00$ & 3.11E+01 & $(2.53 \mathrm{E}-01)$ & $1.36 \mathrm{E}+01$ & $4.06 \mathrm{E}+00$ \\
\hline $\mathrm{B} 1 \mathrm{P} 3 \mathrm{H} 2 \mathrm{~B}$ & C5602 & 82.8 & $<1.96 \mathrm{E}+00$ & $2.09 \mathrm{E}+01$ & $3.49 \mathrm{E}-01$ & $1.17 \mathrm{E}+01$ & $4.72 \mathrm{E}+00$ \\
\hline B1P3H2A & C5602 & 83.3 & $<9.26 \mathrm{E}+00$ & $(1.16 \mathrm{E}+01)$ & $3.83 \mathrm{E}-01$ & $1.23 \mathrm{E}+01$ & $4.21 \mathrm{E}+00$ \\
\hline B1PBB0B & C5602 & 91.8 & $1.42 \mathrm{E}+01$ & 4.97E-01 & $3.49 \mathrm{E}-01$ & $1.09 \mathrm{E}+01$ & $4.14 \mathrm{E}+00$ \\
\hline B1PBB0A & C5602 & 92.3 & $<8.36 \mathrm{E}+00$ & $3.86 \mathrm{E}-01$ & $(1.67 \mathrm{E}-01)$ & $8.74 \mathrm{E}+00$ & $3.52 \mathrm{E}+00$ \\
\hline B1PBB1B & C5606 & 51.8 & $<9.28 \mathrm{E}+00$ & $6.02 \mathrm{E}-01$ & $1.55 \mathrm{E}-01$ & $1.19 \mathrm{E}+01$ & $4.74 \mathrm{E}+00$ \\
\hline
\end{tabular}




\begin{tabular}{|c|c|c|c|c|c|c|c|}
\hline $\begin{array}{l}\text { Sample } \\
\text { ID }\end{array}$ & $\begin{array}{l}\text { Probe } \\
\text { Hole } \\
\text { ID }\end{array}$ & $\begin{array}{l}\text { Mid- } \\
\text { Depth } \\
\text { ft bgs }\end{array}$ & $\begin{array}{c}\text { Technetium- } \\
99 \\
\text { pCi } / \mathrm{g}\end{array}$ & $\begin{array}{c}\text { Uranium- } \\
238 \\
\mu \mathrm{g} / \mathrm{g}\end{array}$ & $\begin{array}{c}\text { Molybdenum } \\
\mu \mathrm{g} / \mathrm{g}\end{array}$ & $\begin{array}{c}\text { Chromium } \\
\mu \mathrm{g} / \mathrm{g}\end{array}$ & $\begin{array}{l}\text { Lead } \\
\mu \mathrm{g} / \mathrm{g}\end{array}$ \\
\hline "B1PBB1A & "C5606 & $\bar{~} 52.3$ & 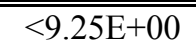 & $101.35 E+00$ & $\begin{array}{c}2.47 \mathrm{E}-01 \\
\end{array}$ & " $1.61 \mathrm{E}+01$ & $=5.29 \mathrm{E}+00$ \\
\hline B1PBB1A DUP & C5606 & 52.3 & $<9.31 \mathrm{E}+00$ & $1.36 E+00$ & $2.78 \mathrm{E}-01$ & $1.71 \mathrm{E}+01$ & $5.52 \mathrm{E}+00$ \\
\hline B1PK51B & C5608 & 64.3 & $<9.08 \mathrm{E}+00$ & $4.00 \mathrm{E}-01$ & $1.88 \mathrm{E}-01$ & $1.97 \mathrm{E}+01$ & $4.86 \mathrm{E}+00$ \\
\hline B1PK51A & C5608 & 64.8 & $<8.84 \mathrm{E}+00$ & $3.32 \mathrm{E}-01$ & $3.76 \mathrm{E}-01$ & $2.30 \mathrm{E}+01$ & $3.78 \mathrm{E}+00$ \\
\hline B1PK52B & C5608 & 85.8 & $5.44 \mathrm{E}+01$ & $3.72 \mathrm{E}-01$ & $5.25 \mathrm{E}-01$ & $5.59 \mathrm{E}+01$ & $5.39 \mathrm{E}+00$ \\
\hline B1PK52A & C5608 & 86.3 & $4.63 E+01$ & $3.64 \mathrm{E}-01$ & $3.85 \mathrm{E}-01$ & $4.52 \mathrm{E}+01$ & $4.83 \mathrm{E}+00$ \\
\hline B1PK53B & C5608 & 97.8 & $5.67 \mathrm{E}+01$ & $6.58 \mathrm{E}-01$ & $4.19 \mathrm{E}-01$ & $5.15 E+01$ & $4.85 \mathrm{E}+00$ \\
\hline B1PK53A & C5608 & 98.3 & $2.36 \mathrm{E}+01$ & $5.37 \mathrm{E}-01$ & $3.34 \mathrm{E}-01$ & $3.16 \mathrm{E}+01$ & $3.91 \mathrm{E}+00$ \\
\hline
\end{tabular}

Comparison of the water to acid-extractable quantities of each constituent was performed by taking the data in Tables 4.22 through 4.24 and dividing them by the data in Tables 4.28 through 4.30 . The data are not presented herein, but show that less than $0.1 \%$ of the acid-extractable quantities of the following elements were water leachable: aluminum, iron, magnesium, manganese, and titanium. Less than $0.5 \%$ of the acid-extractable quantities of the following elements were water leachable: barium, calcium, copper, potassium, nickel, phosphorous (as phosphate), strontium, and zinc. Less than $1 \%$ of the acid-extractable molybdenum was water-extractable. Less than $10 \%$ of the acid-extractable silicon was water-extractable. Less than $15 \%$ of the acid-extractable chromium and sulfur (as sulfate) were water extractable. Finally, less than $30 \%$ of the acid-extractable sodium was water-extractable.

We find it intriguing that elevated concentrations of technetium-99 tend to correlate with unusually high concentrations of chromium. High concentrations of technetium-99 do not correlate with uranium238, but this is expected because partition coefficients for uranium and technetium are quite different in these sediments. Partition coefficients for chromium and technetium also predict that the two elements should be fractionated, so it is enigmatic that we find technetium and chromium together in the same samples. This unusual association may be completely fortuitous, but the correlation may be indicative of a co-precipitation mechanism. In oxidizing conditions chromium is mobilized as the chromate ion $\left[\mathrm{Cr}(\mathrm{VI}) \mathrm{O}_{4}{ }^{2-}\right]$ and technetium as the pertechnetate anion $\left[\mathrm{Tc}(\mathrm{VII}) \mathrm{O}_{4}{ }^{-}\right]$. Previous investigations have revealed that under favorable circumstances, Tc(VII) can be reduced to $\mathrm{Tc}(\mathrm{IV})$ and incorporated into Fe(III) oxyhydroxide phases (Fredrickson et al., 2004; Zachara et al., 2007). This substitution makes crystallographic sense because the ionic radii of Tc(IV) and Fe(III) are nearly the same (78.5 picometers versus 69 or 78.5 picometers for iron in the low- and high-spin states, respectively). [These values are for cations in 6-fold coordination (Shannon 1976)]. Reduction of $\mathrm{Cr}(\mathrm{VI})$ to $\mathrm{Cr}(\mathrm{III})$ results in a cation with a radius of $75.5 \mathrm{pm}$ (6-fold coordination), which is nearly the same as that of $\mathrm{Tc}(\mathrm{IV})$ and $\mathrm{Fe}(\mathrm{III})$.

Therefore, technetium may co-precipitate not only with iron, but with chromium as well. It may be the case that for chromium co-disposed with technetium that the two elements will partition into the same $\mathrm{Fe}(\mathrm{III})$ oxyhydroxide phases. This would make searches for the site of technetium incorporation much easier, since chromium is easier to detect in mineral phases compared to technetium, which is typically present in trace quantities. We hasten to say, however, that all of this is merely intriguing speculation and a detailed mineralogical study would have to be conducted to ascertain the veracity of this theory. 


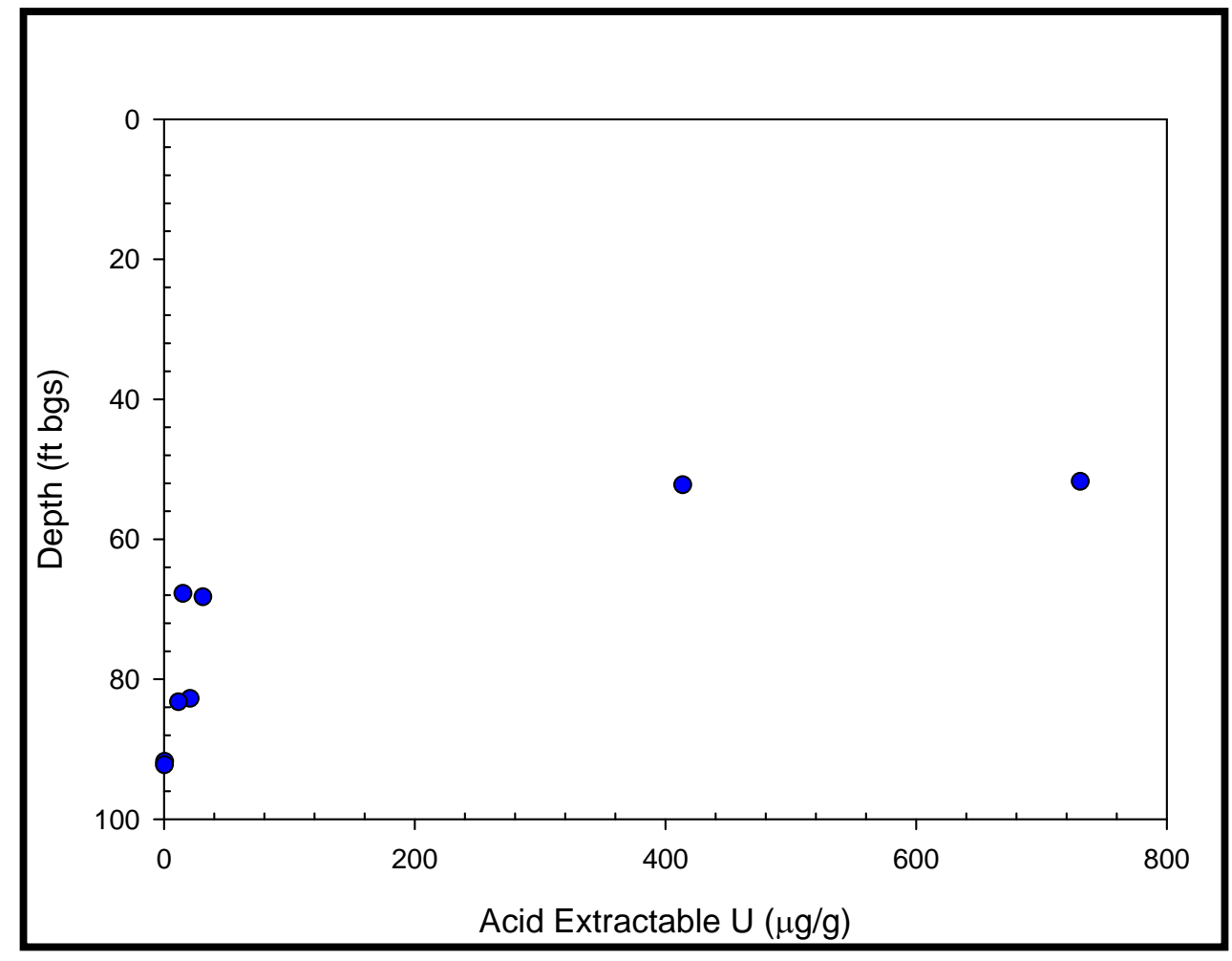

Figure 4.5. Acid-Extractable Uranium-238 Data from Direct Push Hole C5602 Samples

\subsubsection{Radionuclide Content in Vadose Zone Sediment from the U Tank Farm Direct Push Holes Determined by GEA and Gross Alpha and Beta Analysis}

Data from the gamma energy analysis (GEA) of the samples are shown in Table 4.31. The direct measurement of sediment samples for gamma-emitting radionuclides showed that the sediments contained natural potassium-40 in all of the direct push holes. Additionally, contaminant uranium was found in two samples retrieved from push hole C5602. The two samples that contained measurable uranium activity, measured as the protactinium $-234^{\mathrm{m}}$ daughter product at $1001 \mathrm{kev}$, were the two sediments that contained the highest concentration of acid-extractable uranium. Comparison of the acidextractable uranium data vs. that acquired via GEA of the solids for samples B1P3H0B and B1P3H0A revealed that the value obtained by the GEA data was higher in both samples. GEA of sample B1P3H0B determined a uranium concentration of $904 \mu \mathrm{g} / \mathrm{g}$, vs. $731 \mu \mathrm{g} / \mathrm{g}$ measured in the acid extract. Likewise, GEA of sample B1P3H0A measured a uranium concentration of $550 \mu \mathrm{g} / \mathrm{g}$, vs. $414 \mu \mathrm{g} / \mathrm{g}$ determined in the acid extract. Percent differences between the two analyses for samples B1P3H0B and B1P3H0A were $21.2 \%$ and $28.2 \%$, respectively. These results likely indicate that the acid extraction technique was not entirely efficient at removing the contaminant uranium from the sample, leading to higher concentrations when the entire solid was analyzed via GEA. As previously noted, background concentrations of uranium in Hanford Sediments are approximately $3 \mu \mathrm{g} / \mathrm{g}$ or less, indicating that the difference between the two measurements can not merely be an artifact of natural uranium that is recalcitrant to leaching. 
Table 4.31. Gamma-Emitting Radionuclides in the U Tank Farm Direct Push Sediments

\begin{tabular}{|c|c|c|c|c|c|c|}
\hline $\begin{array}{l}\text { Sample } \\
\text { ID }\end{array}$ & $\begin{array}{l}\text { Probe Hole } \\
\text { ID }\end{array}$ & $\begin{array}{l}\text { Mid-Depth } \\
\mathrm{ft} \text { bgs }\end{array}$ & $\begin{array}{c}\text { Potassium-40 } \\
\mathrm{pCi} / \mathrm{g}\end{array}$ & $\begin{array}{l}\text { Error } \\
\mathrm{pCi} / \mathrm{g}\end{array}$ & $\begin{array}{c}\text { Uranium-238 } \\
\text { pCi } / g\end{array}$ & $\begin{array}{l}\text { Error } \\
\mathrm{pCi} / \mathrm{g}\end{array}$ \\
\hline B1NDW3B & C5590 & 96.3 & $2.53 \mathrm{E}+01$ & $1.66 \mathrm{E}+00$ & $<3.93 \mathrm{E}+01$ & $\mathrm{NA}$ \\
\hline B1NDW3A & C5590 & 96.8 & $1.78 \mathrm{E}+01$ & $1.50 \mathrm{E}+00$ & $<5.74 \mathrm{E}+01$ & $\mathrm{NA}$ \\
\hline B1NHV0B & C5592 & 62.3 & $2.22 \mathrm{E}+01$ & $1.90 \mathrm{E}+00$ & $<5.77 \mathrm{E}+01$ & NA \\
\hline B1NHV0A & C5592 & 62.8 & $2.32 \mathrm{E}+01$ & $1.64 \mathrm{E}+00$ & $<3.52 \mathrm{E}+01$ & $\mathrm{NA}$ \\
\hline B1NTC6B & C5598 & 50.3 & $2.42 \mathrm{E}+01$ & $1.37 \mathrm{E}+00$ & $<4.74 \mathrm{E}+01$ & $\mathrm{NA}$ \\
\hline B1NTC6A & C5598 & $\begin{array}{l}50.8 \\
\end{array}$ & $2.19 \mathrm{E}+01$ & $1.63 \mathrm{E}+00$ & $<4.19 \mathrm{E}+01$ & $\mathrm{NA}$ \\
\hline B1NTC7B & C5598 & 59.8 & $2.81 \mathrm{E}+01$ & $1.55 \mathrm{E}+00$ & $<3.37 \mathrm{E}+01$ & NA \\
\hline B1NTC7A & C5598 & 60.3 & $2.24 \mathrm{E}+01$ & $1.39 \mathrm{E}+00$ & $<4.88 \mathrm{E}+01$ & $\mathrm{NA}$ \\
\hline B1NTC8B & C5598 & 82.3 & $2.43 \mathrm{E}+01$ & $1.60 \mathrm{E}+00$ & $<4.78 \mathrm{E}+01$ & $\mathrm{NA}$ \\
\hline B1NTC8A & C5598 & 82.8 & $2.28 \mathrm{E}+01$ & $1.53 \mathrm{E}+00$ & $<4.31 \mathrm{E}+01$ & NA \\
\hline B1NTC9B & C5596 & 50.8 & $2.40 \mathrm{E}+01$ & $1.38 \mathrm{E}+00$ & $<3.09 \mathrm{E}+01$ & NA \\
\hline B1NTC9A & C5596 & 51.3 & $2.42 \mathrm{E}+01$ & $1.56 \mathrm{E}+00$ & $<4.85 \mathrm{E}+01$ & NA \\
\hline B1NTD0B & C5596 & 61.3 & $2.42 \mathrm{E}+01$ & $1.58 \mathrm{E}+00$ & $<4.25 \mathrm{E}+01$ & NA \\
\hline B1NTD0A & C5596 & 61.8 & $2.31 \mathrm{E}+01$ & $1.34 \mathrm{E}+00$ & $<2.79 \mathrm{E}+01$ & NA \\
\hline B1NTD1B & C5596 & 77.8 & $2.20 \mathrm{E}+01$ & $1.65 \mathrm{E}+00$ & $<6.24 \mathrm{E}+01$ & NA \\
\hline B1NTD1A & C5596 & 78.3 & $2.38 \mathrm{E}+01$ & $1.67 \mathrm{E}+00$ & $<4.76 \mathrm{E}+01$ & NA \\
\hline B1NTD2B & C5596 & 82.8 & $2.19 \mathrm{E}+01$ & $1.22 \mathrm{E}+00$ & $<2.56 \mathrm{E}+01$ & $\mathrm{NA}$ \\
\hline B1NTD2A & C5596 & 83.3 & $2.31 \mathrm{E}+01$ & $1.33 \mathrm{E}+00$ & $<4.44 \mathrm{E}+01$ & NA \\
\hline B1NTD3B & C5600 & 50.3 & $1.93 \mathrm{E}+01$ & $1.55 \mathrm{E}+00$ & $<5.03 \mathrm{E}+01$ & NA \\
\hline B1NTD3A & C5600 & 50.8 & $2.18 \mathrm{E}+01$ & $1.60 \mathrm{E}+00$ & $<3.83 \mathrm{E}+01$ & NA \\
\hline B1NTD4B & C5600 & 60.3 & $2.15 \mathrm{E}+01$ & $1.49 \mathrm{E}+00$ & $<4.99 \mathrm{E}+01$ & NA \\
\hline B1NTD4A & $\mathrm{C} 5600$ & 60.8 & $2.41 \mathrm{E}+01$ & $1.65 \mathrm{E}+00$ & $<5.31 \mathrm{E}+01$ & NA \\
\hline B1NTD5B & C5600 & 82.3 & $2.54 \mathrm{E}+01$ & $1.65 \mathrm{E}+00$ & $<3.88 \mathrm{E}+01$ & NA \\
\hline B1NTD5A & C5600 & 82.8 & $2.32 \mathrm{E}+01$ & $1.47 \mathrm{E}+00$ & $<4.95 \mathrm{E}+01$ & NA \\
\hline B1P1K6B & $\mathrm{C} 5600$ & 88.8 & $2.15 \mathrm{E}+01$ & $1.51 \mathrm{E}+00$ & $<4.54 \mathrm{E}+01$ & NA \\
\hline B1P1K6A & C5600 & 89.3 & $2.34 \mathrm{E}+01$ & $1.52 \mathrm{E}+00$ & $<3.56 \mathrm{E}+01$ & NA \\
\hline B1P3F9B & C5604 & 50.8 & $2.26 \mathrm{E}+01$ & $1.69 \mathrm{E}+00$ & $<6.39 \mathrm{E}+01$ & NA \\
\hline B1P3F9A & C5604 & 51.3 & $2.33 \mathrm{E}+01$ & $1.61 \mathrm{E}+00$ & $<4.96 \mathrm{E}+01$ & NA \\
\hline B1P3H0B & C5602 & 51.8 & $2.13 \mathrm{E}+01$ & $1.41 \mathrm{E}+00$ & $3.04 \mathrm{E}+02$ & $3.17 \mathrm{E}+01$ \\
\hline B1P3H0A & C5602 & 52.3 & $2.35 \mathrm{E}+01$ & $1.36 \mathrm{E}+00$ & $1.85 \mathrm{E}+02$ & $3.30 \mathrm{E}+01$ \\
\hline B1P3H1B & C5602 & 67.8 & $2.20 \mathrm{E}+01$ & $1.49 \mathrm{E}+00$ & $<4.40 \mathrm{E}+01$ & NA \\
\hline B1P3H1A & C5602 & 68.3 & $2.28 \mathrm{E}+01$ & $1.62 \mathrm{E}+00$ & $<3.86 \mathrm{E}+01$ & NA \\
\hline $\mathrm{B} 1 \mathrm{P} 3 \mathrm{H} 2 \mathrm{~B}$ & C5602 & 82.8 & $2.20 \mathrm{E}+01$ & $1.38 \mathrm{E}+00$ & $<5.53 \mathrm{E}+01$ & $\mathrm{NA}$ \\
\hline B1P3H2A & C5602 & 83.3 & $2.15 \mathrm{E}+01$ & $1.52 \mathrm{E}+00$ & $<4.59 \mathrm{E}+01$ & NA \\
\hline B1PBB0B & C5602 & 91.8 & $2.18 \mathrm{E}+01$ & $1.65 \mathrm{E}+00$ & $<3.69 \mathrm{E}+01$ & $\mathrm{NA}$ \\
\hline B1PBB0A & C5602 & 92.3 & $2.18 \mathrm{E}+01$ & $1.57 \mathrm{E}+00$ & $<5.63 \mathrm{E}+01$ & $\mathrm{NA}$ \\
\hline B1PBB1B & C5606 & 51.8 & $2.26 \mathrm{E}+01$ & $1.40 \mathrm{E}+00$ & $<3.85 \mathrm{E}+01$ & NA \\
\hline B1PBB1A & C5606 & 52.3 & $2.34 \mathrm{E}+01$ & $1.71 \mathrm{E}+00$ & $<5.13 \mathrm{E}+01$ & NA \\
\hline B1PK51B & C5608 & 64.3 & $2.36 \mathrm{E}+01$ & $1.81 \mathrm{E}+00$ & $<6.45 \mathrm{E}+01$ & NA \\
\hline B1PK51A & C5608 & 64.8 & $2.26 \mathrm{E}+01$ & $1.71 \mathrm{E}+00$ & $<4.52 \mathrm{E}+01$ & NA \\
\hline B1PK52B & C5608 & 85.8 & $2.37 \mathrm{E}+01$ & $1.32 \mathrm{E}+00$ & $<3.44 \mathrm{E}+01$ & $\mathrm{NA}$ \\
\hline B1PK52A & C5608 & 86.3 & $2.40 \mathrm{E}+01$ & $1.64 \mathrm{E}+00$ & $<5.40 \mathrm{E}+01$ & NA \\
\hline B1PK53B & C5608 & 97.8 & $1.99 \mathrm{E}+01$ & $1.87 \mathrm{E}+00$ & $<6.03 \mathrm{E}+01$ & NA \\
\hline B1PK53A & C5608 & 98.3 & $2.33 \mathrm{E}+01$ & $1.37 \mathrm{E}+00$ & $<3.60 \mathrm{E}+01$ & $\mathrm{NA}$ \\
\hline
\end{tabular}


Comparison of the laboratory-generated GEA data vs. that acquired in the field by Randall and Price (2007) had limited success. The comparison was made specifically for samples from push hole C5602, which correlated with logging data from push hole C5601 (these were the only companion holes that had laboratory samples from a depth identified via field logging as containing gamma-emitting radionuclide elements). Gross gamma logging data acquired in push hole C5601 identified a peak activity at $52 \mathrm{ft}$ bgs, with an equivalent cesium-137 activity of $24 \mathrm{pCi} / \mathrm{g}$. As shown in Table 4.31, sediment retrieved from approximately $52 \mathrm{ft}$ bgs in push hole $\mathrm{C} 5602$ contained in excess of $300 \mathrm{pCi} / \mathrm{g}$ contaminant uranium. Although the sodium iodide detector was effective at identifying a region of elevated gamma activity in the push hole, it should have resulted in additional analysis using the spectral gamma logging tool. The only other two probe holes (C5593 and C5607) that had detectable gross gamma activity were logged using the spectral gamma tool. However, laboratory samples were not collected from their companion probe holes (C5594 and C5608); therefore, an assessment of the efficiency of the spectral gamma tool could not be made.

Gross alpha and beta measurements were made on aliquots of the water and acid extracts. Gross alpha activity was not detected in any of the water extract samples; however, gross beta activity was detected in four samples from push C5608. Table 4.32 contains a comparison of the gross beta activity measured via liquid scintillation counting vs. technetium-99 measured via ICP-MS. Comparison of the data generated via the two analytical methods was quite good ( $10 \%$ to $12 \%$ relative difference) for the two samples retrieved from approximately $86 \mathrm{ft}$ bgs. The percent relative differences for the two samples retrieved from approximately $98 \mathrm{ft}$ bgs ranged from $32 \%-40 \%$. Based on this, it appears that a mobile beta-emitter, likely tritium, is contributing to the gross beta activity in the samples from approximately $98 \mathrm{ft}$ bgs.

Table 4.32. Gross Beta vs. ICP-MS Data in the U Tank Farm Direct Push Water Extracts

\begin{tabular}{||c|c|c|c|c|c||}
\hline $\begin{array}{c}\text { Sample } \\
\text { ID }\end{array}$ & $\begin{array}{c}\text { Probe Hole } \\
\text { ID }\end{array}$ & $\begin{array}{c}\text { Mid-Depth } \\
\mathrm{ft} \text { bgs }\end{array}$ & $\begin{array}{c}\text { Gross Beta } \\
\text { pCi/g }\end{array}$ & $\begin{array}{c}\text { Technetium-99 } \\
\text { pCi/g }\end{array}$ & $\begin{array}{c}\text { Percent Relative } \\
\text { Difference }\end{array}$ \\
\hline \hline B1PK52B & C5608 & 85.8 & $5.48 \mathrm{E}+01$ & $4.88 \mathrm{E}+01$ & 11.59 \\
\hline B1PK52A & C5608 & 86.3 & $4.67 \mathrm{E}+01$ & $4.19 \mathrm{E}+01$ & 10.76 \\
\hline B1PK53B & C5608 & 97.8 & $2.37 \mathrm{E}+01$ & $1.59 \mathrm{E}+01$ & 39.38 \\
\hline B1PK53A & C5608 & 98.3 & $1.46 \mathrm{E}+01$ & $1.05 \mathrm{E}+01$ & 32.49 \\
\hline
\end{tabular}

Gross alpha and beta activity was detected in acid extracts of two of the U Farm direct push samples (Table 4.33). These results could not be correlated with any of the radionuclide elements measured in the samples using alternate techniques; therefore, the samples were analyzed for neptunium-237, plutonium239, and americium-241 via ICP-MS. ICP-MS analysis of the samples enabled us to rule out neptunium237 and plutonium-239 as potential sources of the gross beta activity. The estimated quantification limit for americium-241 in the samples via ICP-MS analysis was above the reported gross alpha and beta data; therefore, americium-241 could not be ruled out as a potential source of the contamination in the samples.

Table 4.33. Gross Alpha and Beta Data in the U Tank Farm Direct Push Acid Extracts

\begin{tabular}{||c|c|c|c|c|c|c||}
\hline $\begin{array}{c}\text { Sample } \\
\text { ID }\end{array}$ & $\begin{array}{c}\text { Probe Hole } \\
\text { ID }\end{array}$ & $\begin{array}{c}\text { Mid-Depth } \\
\mathrm{ft} \text { bgs }\end{array}$ & $\begin{array}{c}\text { Gross Beta } \\
\text { pCi/g }\end{array}$ & $\begin{array}{c}\text { Error } \\
\text { pCi/g) }\end{array}$ & $\begin{array}{c}\text { Gross Alpha } \\
\text { pCi/g }\end{array}$ & $\begin{array}{c}\text { Error } \\
\text { pCi } / g\end{array}$ \\
\hline \hline B1P3H0B & C5602 & 51.8 & $1.508 \mathrm{E}+03$ & $1.551 \mathrm{E}+02$ & $1.554 \mathrm{E}+02$ & $4.829 \mathrm{E}+01$ \\
\hline B1P3H0A & C5602 & 52.3 & $3.456 \mathrm{E}+02$ & $8.653 \mathrm{E}+01$ & $2.402 \mathrm{E}+02$ & $5.531 \mathrm{E}+01$ \\
\hline
\end{tabular}




\subsubsection{Total Carbon, Calcium Carbonate, and Organic Carbon Content of Vadose Zone Sediment from the U Tank Farm Direct Push Holes}

Data from the total carbon, inorganic carbon, and organic carbon (calculated by difference) contents of the U Tank Farm direct push sediments are shown in Table 4.34. The inorganic carbon was converted to the equivalent calcium carbonate content. Organic carbon was not quantified in any of the $U$ farm direct push samples; the estimated quantification limit was $0.03 \mathrm{wt} \%$. Inorganic carbon, as $\mathrm{CaCO}_{3}$, was present in the samples at concentrations that are typical for Hanford sediments ( $1 \mathrm{wt} \%$ to $3.5 \mathrm{wt} \%$ as $\mathrm{CaCO}_{3}$ ) and compare well with samples from the background borehole and uncontaminated locations (Serne et al. 2004a,b).

Table 4.34. Total, Inorganic, and Organic Carbon Content of Vadose Zone Sediments from the Direct Push Holes

\begin{tabular}{|c|c|c|c|c|c|c|}
\hline $\begin{array}{c}\text { Sample } \\
\text { ID }\end{array}$ & $\begin{array}{c}\text { Probe Hole } \\
\text { ID }\end{array}$ & $\begin{array}{c}\text { Mid-Depth } \\
\text { ft bgs }\end{array}$ & $\begin{array}{c}\text { Total } \\
\text { Carbon } \\
(\%) \\
\end{array}$ & $\begin{array}{c}\text { Inorganic } \\
\text { Carbon } \\
(\%) \\
\end{array}$ & $\begin{array}{c}\text { Inorganic } \\
\text { Carbon } \\
\text { as } \mathrm{CaCO}_{3} \\
(\%) \\
\end{array}$ & $\begin{array}{c}\text { Organic } \\
\text { Carbon } \\
\quad(\text { by } \\
\text { difference) }\end{array}$ \\
\hline B1NDW3B & C5590 & 96.3 & $2.96 \mathrm{E}-01$ & $2.10 \mathrm{E}-01$ & $1.75 \mathrm{E}+00$ & $<2<2.69 \mathrm{E}-02$ \\
\hline B1NDW3A & C5590 & 96.8 & $2.62 \mathrm{E}-01$ & $2.11 \mathrm{E}-01$ & $1.76 \mathrm{E}+00$ & $<2.69 \mathrm{E}-02$ \\
\hline B1NDW3A DUP & C5590 & 96.8 & $2.48 \mathrm{E}-01$ & $1.87 \mathrm{E}-01$ & $1.56 \mathrm{E}+00$ & $<2.69 \mathrm{E}-02$ \\
\hline B1NHV0B & C5592 & 62.3 & $2.79 \mathrm{E}-01$ & $2.37 \mathrm{E}-01$ & $1.98 \mathrm{E}+00$ & $<2.69 \mathrm{E}-02$ \\
\hline B1NHV0A & C5592 & 62.8 & $2.54 \mathrm{E}-01$ & $1.83 \mathrm{E}-01$ & $1.52 \mathrm{E}+00$ & $<2.69 \mathrm{E}-02$ \\
\hline B1NTC6B & C5598 & 50.3 & $3.15 \mathrm{E}-01$ & $2.82 \mathrm{E}-01$ & $2.35 \mathrm{E}+00$ & $<2.69 \mathrm{E}-02$ \\
\hline B1NTC6A & C5598 & 50.8 & $3.76 \mathrm{E}-01$ & $3.53 \mathrm{E}-01$ & $2.94 \mathrm{E}+00$ & $<2.69 \mathrm{E}-02$ \\
\hline B1NTC7B & C5598 & 59.8 & $3.50 \mathrm{E}-01$ & $3.01 \mathrm{E}-01$ & $2.51 \mathrm{E}+00$ & $<2.69 \mathrm{E}-02$ \\
\hline B1NTC7A & C5598 & 60.3 & $2.85 \mathrm{E}-01$ & $2.61 \mathrm{E}-01$ & $2.18 \mathrm{E}+00$ & $<2.69 \mathrm{E}-02$ \\
\hline B1NTC8B & C5598 & 82.3 & 3.04E-01 & $2.42 \mathrm{E}-01$ & $2.02 \mathrm{E}+00$ & $<2.69 \mathrm{E}-02$ \\
\hline B1NTC8B DUP & C5598 & 82.3 & $2.72 \mathrm{E}-01$ & $2.31 \mathrm{E}-01$ & $1.93 \mathrm{E}+00$ & $<2.69 \mathrm{E}-02$ \\
\hline B1NTC8A & C5598 & 82.8 & $2.95 \mathrm{E}-01$ & $2.53 \mathrm{E}-01$ & $2.11 \mathrm{E}+00$ & $<2.69 \mathrm{E}-02$ \\
\hline B1NTC9B & C5596 & 50.8 & $3.30 \mathrm{E}-01$ & $3.01 \mathrm{E}-01$ & $2.51 \mathrm{E}+00$ & $<2.69 \mathrm{E}-02$ \\
\hline B1NTC9A & C5596 & 51.3 & 3.09E-01 & $2.77 \mathrm{E}-01$ & $2.31 \mathrm{E}+00$ & $<2.69 \mathrm{E}-02$ \\
\hline B1NTD0B & C5596 & 61.3 & $3.08 \mathrm{E}-01$ & $2.53 \mathrm{E}-01$ & $2.11 \mathrm{E}+00$ & $<2.69 \mathrm{E}-02$ \\
\hline B1NTD0A & C5596 & 61.8 & $2.86 \mathrm{E}-01$ & $2.40 \mathrm{E}-01$ & $2.00 \mathrm{E}+00$ & $<2.69 \mathrm{E}-02$ \\
\hline B1NTD1B & C5596 & 77.8 & $2.55 \mathrm{E}-01$ & $2.12 \mathrm{E}-01$ & $1.76 \mathrm{E}+00$ & $<2.69 \mathrm{E}-02$ \\
\hline B1NTD1B DUP & C5596 & 77.8 & $2.59 \mathrm{E}-01$ & $2.31 \mathrm{E}-01$ & $1.93 \mathrm{E}+00$ & $<2.69 \mathrm{E}-02$ \\
\hline B1NTD1A & C5596 & 78.3 & $2.69 \mathrm{E}-01$ & $2.21 \mathrm{E}-01$ & $1.84 \mathrm{E}+00$ & $<2.69 \mathrm{E}-02$ \\
\hline B1NTD2B & C5596 & 82.8 & $2.56 \mathrm{E}-01$ & $2.25 \mathrm{E}-01$ & $1.87 \mathrm{E}+00$ & $<2.69 \mathrm{E}-02$ \\
\hline B1NTD2A & C5596 & 83.3 & $2.44 \mathrm{E}-01$ & $2.06 \mathrm{E}-01$ & $1.72 \mathrm{E}+00$ & $<2.69 \mathrm{E}-02$ \\
\hline B1NTD3B & C5600 & 50.3 & $3.38 \mathrm{E}-01$ & $3.23 \mathrm{E}-01$ & $2.69 \mathrm{E}+00$ & $<2.69 \mathrm{E}-02$ \\
\hline B1NTD3A & C5600 & 50.8 & $3.51 \mathrm{E}-01$ & $3.27 \mathrm{E}-01$ & $2.73 \mathrm{E}+00$ & $<2.69 \mathrm{E}-02$ \\
\hline B1NTD4B & C5600 & 60.3 & $2.78 \mathrm{E}-01$ & $2.26 \mathrm{E}-01$ & $1.89 \mathrm{E}+00$ & $<2.69 \mathrm{E}-02$ \\
\hline B1NTD4A & C5600 & 60.8 & $2.64 \mathrm{E}-01$ & $2.04 \mathrm{E}-01$ & $1.70 \mathrm{E}+00$ & $<2.69 \mathrm{E}-02$ \\
\hline B1NTD5B & C5600 & 82.3 & $2.97 \mathrm{E}-01$ & $2.47 \mathrm{E}-01$ & $2.06 \mathrm{E}+00$ & $<2.69 \mathrm{E}-02$ \\
\hline B1NTD5A & C5600 & 82.8 & $2.72 \mathrm{E}-01$ & $2.24 \mathrm{E}-01$ & $1.87 \mathrm{E}+00$ & $<2.69 \mathrm{E}-02$ \\
\hline B1P1K6B & C5600 & 88.8 & $2.20 \mathrm{E}-01$ & $1.70 \mathrm{E}-01$ & $1.41 \mathrm{E}+00$ & $<2.69 \mathrm{E}-02$ \\
\hline B1P1K6A & C5600 & 89.3 & $2.33 \mathrm{E}-01$ & $1.86 \mathrm{E}-01$ & $1.55 \mathrm{E}+00$ & $<2.69 \mathrm{E}-02$ \\
\hline B1P3F9B & C5604 & 50.8 & $3.41 \mathrm{E}-01$ & $3.06 \mathrm{E}-01$ & $2.55 \mathrm{E}+00$ & $<2.69 \mathrm{E}-02$ \\
\hline
\end{tabular}




\begin{tabular}{||l|c|c|c|c|c|c||}
\hline $\begin{array}{c}\text { Sample } \\
\text { ID }\end{array}$ & $\begin{array}{c}\text { Probe Hole } \\
\text { ID }\end{array}$ & $\begin{array}{c}\text { Mid-Depth } \\
\text { ft bgs }\end{array}$ & $\begin{array}{c}\text { Total } \\
\text { Carbon } \\
(\%)\end{array}$ & $\begin{array}{c}\text { Inorganic } \\
\text { Carbon } \\
(\%)\end{array}$ & $\begin{array}{c}\text { Inorganic } \\
\text { Carbon } \\
\text { as CaCO } \\
(\%)\end{array}$ & $\begin{array}{c}\text { Organic } \\
\text { Carbon } \\
\text { (by } \\
\text { difference })\end{array}$ \\
\hline \hline B1P3F9A & C5604 & 51.3 & $2.82 \mathrm{E}-01$ & $2.53 \mathrm{E}-01$ & $2.11 \mathrm{E}+00$ & $<2.69 \mathrm{E}-02$ \\
\hline B1P3H0B & C5602 & 51.8 & $2.81 \mathrm{E}-01$ & $2.53 \mathrm{E}-01$ & $2.11 \mathrm{E}+00$ & $<2.69 \mathrm{E}-02$ \\
\hline B1P3H0A & C5602 & 52.3 & $2.65 \mathrm{E}-01$ & $2.36 \mathrm{E}-01$ & $1.97 \mathrm{E}+00$ & $<2.69 \mathrm{E}-02$ \\
\hline B1P3H1B & C5602 & 67.8 & $2.48 \mathrm{E}-01$ & $2.25 \mathrm{E}-01$ & $1.87 \mathrm{E}+00$ & $<2.69 \mathrm{E}-02$ \\
\hline B1P3H1A & C5602 & 68.3 & $2.41 \mathrm{E}-01$ & $2.13 \mathrm{E}-01$ & $1.78 \mathrm{E}+00$ & $<2.69 \mathrm{E}-02$ \\
\hline B1P3H2B & C5602 & 82.8 & $2.88 \mathrm{E}-01$ & $2.34 \mathrm{E}-01$ & $1.95 \mathrm{E}+00$ & $<2.69 \mathrm{E}-02$ \\
\hline B1P3H2A & C5602 & 83.3 & $2.76 \mathrm{E}-01$ & $2.27 \mathrm{E}-01$ & $1.90 \mathrm{E}+00$ & $<2.69 \mathrm{E}-02$ \\
\hline B1PBB0B & C5602 & 91.8 & $2.28 \mathrm{E}-01$ & $1.86 \mathrm{E}-01$ & $1.55 \mathrm{E}+00$ & $<2.69 \mathrm{E}-02$ \\
\hline B1PBB0A & C5602 & 92.3 & $2.32 \mathrm{E}-01$ & $1.95 \mathrm{E}-01$ & $1.63 \mathrm{E}+00$ & $<2.69 \mathrm{E}-02$ \\
\hline B1PBB1B & C5606 & 51.8 & $2.96 \mathrm{E}-01$ & $3.61 \mathrm{E}-01$ & $3.01 \mathrm{E}+00$ & $<2.69 \mathrm{E}-02$ \\
\hline B1PBB1A & C5606 & 52.3 & $3.39 \mathrm{E}-01$ & $3.95 \mathrm{E}-01$ & $3.29 \mathrm{E}+00$ & $<2.69 \mathrm{E}-02$ \\
\hline B1PBB1A DUP & C5606 & 52.3 & $3.59 \mathrm{E}-01$ & $4.01 \mathrm{E}-01$ & $3.35 \mathrm{E}+00$ & $<2.69 \mathrm{E}-02$ \\
\hline B1PK51B & C5608 & 64.3 & $2.77 \mathrm{E}-01$ & $2.93 \mathrm{E}-01$ & $2.44 \mathrm{E}+00$ & $<2.69 \mathrm{E}-02$ \\
\hline B1PK51A & C5608 & 64.8 & $1.93 \mathrm{E}-01$ & $2.49 \mathrm{E}-01$ & $2.07 \mathrm{E}+00$ & $<2.69 \mathrm{E}-02$ \\
\hline B1PK52B & C5608 & 85.8 & $2.58 \mathrm{E}-01$ & $3.00 \mathrm{E}-01$ & $2.50 \mathrm{E}+00$ & $<2.69 \mathrm{E}-02$ \\
\hline B1PK52A & C5608 & 86.3 & $2.35 \mathrm{E}-01$ & $2.66 \mathrm{E}-01$ & $2.22 \mathrm{E}+00$ & $<2.69 \mathrm{E}-02$ \\
\hline B1PK53B & C5608 & 97.8 & $2.27 \mathrm{E}-01$ & $2.60 \mathrm{E}-01$ & $2.17 \mathrm{E}+00$ & $<2.69 \mathrm{E}-02$ \\
\hline B1PK53A & C5608 & 98.3 & $2.08 \mathrm{E}-01$ & $1.98 \mathrm{E}-01$ & $1.65 \mathrm{E}+00$ & $<2.69 \mathrm{E}-02$ \\
\hline Less-than symbol indicates the instrument returned a negative value. & & & \\
\hline \hline
\end{tabular}

\subsubsection{Particle Size Measurements on U Farm Direct Push Vadose Zone Sediment}

Hydrometer and wet sieving methods were used to determine the particle size distributions of samples from several of the direct push holes. Wet sieving results are shown in Table 4.35 and the particle-size distribution data from both techniques are shown in Table 4.36 and Figure 4.6 as a plot of "cumulative percent finer than" versus "particle size in microns." As seen in Table 4.36, two of the U Farm direct push samples had median grain sizes of approximately 100 microns (B1NTC9 and B1NTD1), two of the samples had median grain sizes of approximately 250 microns (D1NTC7 and B1NTD3C), and the fifth sample (D1NDW3) had a median grain size between 100 and 250 microns. Unlike samples analyzed from the background borehole, all of the direct push samples were collected from the Hanford formation $\mathrm{H} 2$ unit. The moisture content of the five Hanford formation $\mathrm{H} 2$ unit samples ranged between $2.13 \mathrm{wt} \%$ and $11.5 \mathrm{wt} \%$, and like the background sediments analyzed, moisture content could not be correlated with median grain size or percentage of silt/clay present in the samples. For example, the sample containing the second-highest moisture content (B1NTD3C) contained the lowest amount of silt/clay, at $20.6 \mathrm{wt} \%$, and had a median grain size of 250 microns. As with the background sediments, a larger subset of samples should be analyzed for particle size before correlations between moisture content and median particle size or silt/clay content are attempted. 
Table 4.35. Wet Sieve Particle Size Results for U Farm Direct Push Sediments

\begin{tabular}{|c|c|c|c|c|c|c|}
\hline \multirow[b]{2}{*}{ Sample ID } & \multirow{2}{*}{$\begin{array}{l}\text { Mid-Depth } \\
\text { (ft bgs) }\end{array}$} & \multirow{2}{*}{$\begin{array}{c}\text { Moisture Content } \\
(\%)\end{array}$} & \multirow{2}{*}{$\begin{array}{c}\text { Stratigraphic } \\
\text { Unit }\end{array}$} & \multicolumn{3}{|c|}{ Weight Percent } \\
\hline & & & & Gravel & Sand & Silt/Clay \\
\hline B1NDW3 & 97.3 & 7.57 & $\mathrm{H} 2$ & 0 & 78.7 & 21.2 \\
\hline B1NTC9 & 51.8 & 11.5 & $\mathrm{H} 2$ & 0 & 66.2 & 33.8 \\
\hline B1NTD1 & 78.8 & 4.65 & $\overline{\mathrm{H} 2}$ & 0 & 69.7 & 29.9 \\
\hline B1NTC7 & 60.8 & 2.13 & $\mathrm{H} 2$ & 0.599 & 77.1 & 22.0 \\
\hline B1NTD3C & 49.8 & 10.3 & $\mathrm{H} 2$ & 0.022 & 79.4 & 20.6 \\
\hline
\end{tabular}

Table 4.36. Particle Size Data for U Farm Direct Push Sediments Using Two Techniques Reported as Cumulative Percent Finer Than

\begin{tabular}{|c|c|c|c|c|c|c|c|c|c|}
\hline \multicolumn{2}{|c|}{ B1NDW3 } & \multicolumn{2}{|c|}{ B1NTC9 } & \multicolumn{2}{|c|}{ B1NTD1 } & \multicolumn{2}{|c|}{ B1NTC7 } & \multicolumn{2}{|c|}{ B1NTD3C } \\
\hline \multicolumn{2}{|c|}{$\mathrm{H} 2$} & \multicolumn{2}{|c|}{$\mathrm{H} 2$} & \multicolumn{2}{|c|}{$\mathrm{H} 2$} & \multicolumn{2}{|c|}{$\mathrm{H} 2$} & \multicolumn{2}{|c|}{$\mathrm{H} 2$} \\
\hline $\begin{array}{l}\text { Diameter } \\
(\mu \mathrm{m})\end{array}$ & $\begin{array}{c}\% \\
\text { finer than }\end{array}$ & $\begin{array}{l}\text { Diameter } \\
(\mu \mathrm{m})\end{array}$ & $\begin{array}{c}\% \\
\text { finer than }\end{array}$ & $\begin{array}{c}\text { Diameter } \\
(\mu \mathrm{m})\end{array}$ & $\begin{array}{c}\% \\
\text { finer than }\end{array}$ & $\begin{array}{c}\text { Diameter } \\
(\mu \mathrm{m})\end{array}$ & $\begin{array}{c}\% \\
\text { finer than }\end{array}$ & $\begin{array}{l}\text { Diameter } \\
(\mu \mathrm{m})\end{array}$ & $\begin{array}{c}\% \\
\text { finer than }\end{array}$ \\
\hline \multicolumn{10}{|c|}{ Wet Sieve } \\
\hline 2000 & 100 & 2000 & 100 & 2000 & 100 & 2000 & 99.4 & 2000 & 100 \\
\hline 1000 & 100 & 1000 & 98.7 & \begin{tabular}{|l|}
1000 \\
\end{tabular} & 100 & 1000 & 95.9 & 1000 & 98.7 \\
\hline 500 & 99.8 & 500 & 89.1 & 500 & 98.4 & 500 & 75.8 & 500 & 83.8 \\
\hline 250 & 96.1 & 250 & 74.6 & 250 & 86.0 & 250 & 49.3 & 250 & 54.6 \\
\hline 106 & 37.5 & 106 & 56.9 & 106 & 53.8 & 106 & 29.1 & 106 & 33.0 \\
\hline 75 & 25.2 & 75 & 43.1 & 75 & 36.8 & 75 & 25.7 & 75 & 24.7 \\
\hline 53 & 21.3 & 53 & 33.8 & 53 & 30.3 & 53 & 22.3 & 53 & 20.6 \\
\hline \multicolumn{10}{|c|}{ Hydrometer } \\
\hline 85.8 & 24.8 & 86.3 & 41.1 & 86.7 & 33.9 & 83.5 & 23.7 & 88.6 & 23.8 \\
\hline 59.6 & 18.9 & 60.0 & 30.5 & 60.4 & 26.7 & 58.8 & 20.5 & 61.5 & 18.8 \\
\hline 33.8 & 13.1 & 34.2 & 22.6 & 34.7 & 23.6 & 33.8 & 17.4 & 35.0 & 15.0 \\
\hline 18.3 & 10.4 & 18.6 & 18.6 & 18.8 & 18.5 & 18.5 & 15.8 & 19.0 & 12.7 \\
\hline 10.5 & 8.49 & 10.7 & 14.6 & 10.7 & 14.4 & 10.6 & 11.0 & 10.8 & 9.42 \\
\hline 7.40 & 7.18 & 7.51 & 11.9 & 7.58 & 13.4 & 7.48 & 11.0 & 7.64 & 8.86 \\
\hline 6.03 & 6.53 & 6.12 & 10.6 & 6.15 & 10.3 & 6.10 & 9.47 & 6.17 & 6.09 \\
\hline 5.21 & 5.88 & 5.29 & 9.29 & 5.31 & 9.24 & 5.28 & 9.47 & 5.38 & 7.76 \\
\hline 1.49 & 3.92 & 1.52 & 6.63 & 1.52 & 7.19 & 1.52 & 7.89 & 1.54 & 6.65 \\
\hline
\end{tabular}




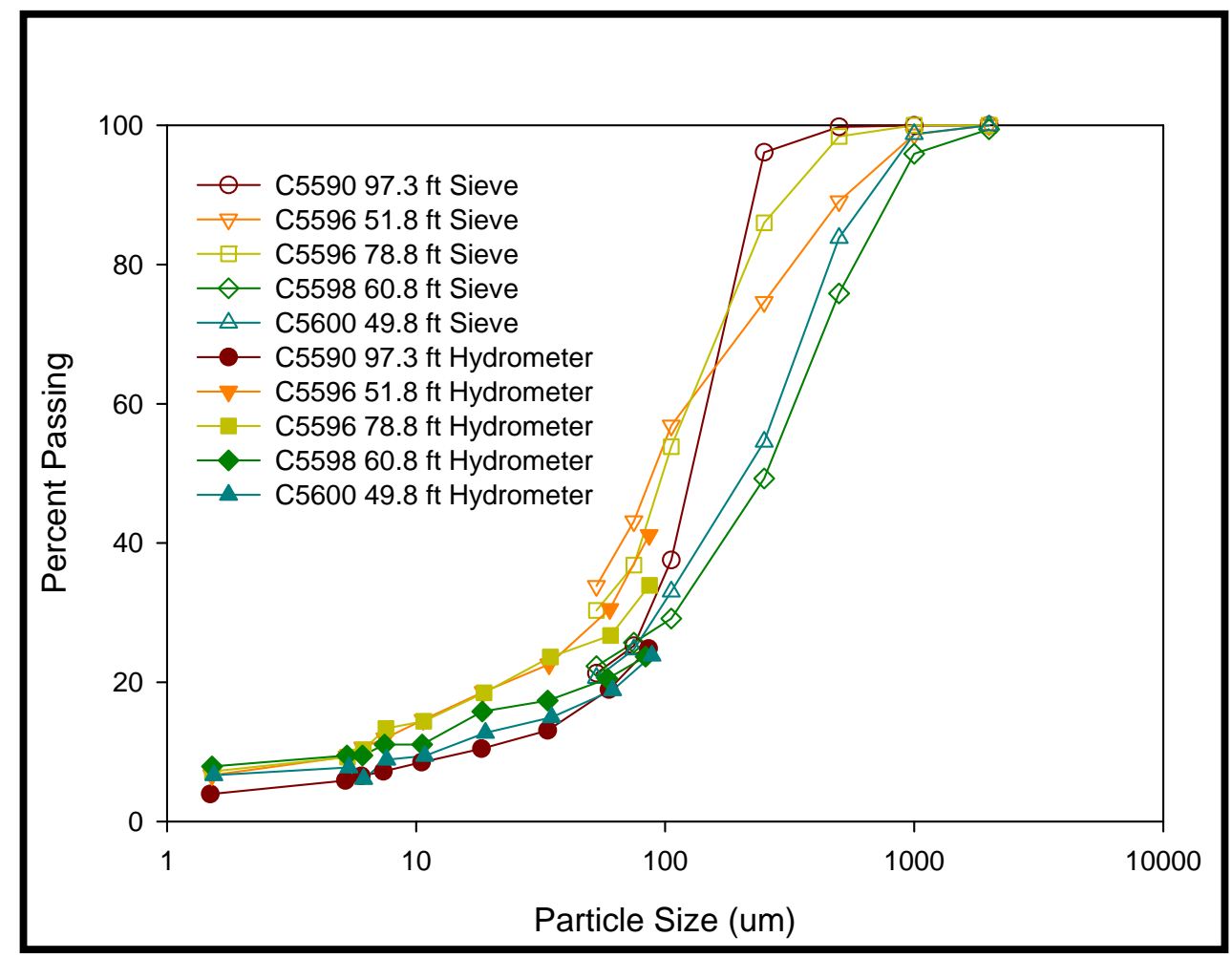

Figure 4.6. 1:1 Sediment:Water-Extractable Uranium-238 Data from Direct Push Hole C5602 Samples

\subsubsection{Ammonium Acetate Extractions}

The exchangeable fraction of cations in samples from the direct push holes is presented in Table 4.37 in units of $\mu \mathrm{g} / \mathrm{g}$ and Table 4.38 in units of meq $/ 100 \mathrm{~g}$ of dry sediment. As with the data generated from the background borehole samples, reproducibility of the measurements, as observed through duplicate analysis of samples, was excellent. Similar to the background borehole sediment, calcium was the dominant ammonium-acetate-extractable cation in all of the direct push samples analyzed. The range in total cation exchange capacities for the direct push samples was slightly narrower than for those measured in the background borehole, at approximately 7 to $9 \mathrm{meq} / 100 \mathrm{~g}$. These results demonstrate that the exchange capacities of the sediments collected within the U Tank Farm do not vary significantly from the capacities of the background borehole sediments. This finding is not surprising given the direct push sample set that was chosen for this analysis. The direct push sampling method generates very small volume sample sizes; therefore, samples that were chosen for particle size analysis (which uses a large sample mass) and CEC analysis were those that were less important from a perspective of contaminant concentration. In the future, it would be advantageous to perform a CEC analysis of samples that clearly contain a significant amount of tank waste contaminants, such as those from push holes C5602 and C5608. 
Table 4.37. Ammonium Acetate-Extractable Cations in the U Farm Direct Push Samples ( $\mu \mathrm{g} / \mathrm{g}$ dry sediment)

\begin{tabular}{||l|c|c|c|c|c|c|c||}
\hline $\begin{array}{c}\text { Sample } \\
\text { ID }\end{array}$ & $\begin{array}{c}\text { Mid-Depth } \\
\mathrm{ft} \text { bgs }\end{array}$ & $\begin{array}{c}\text { Stratigraphic } \\
\text { Unit }\end{array}$ & $\begin{array}{c}\text { Barium } \\
\mu \mathrm{g} / \mathrm{g}\end{array}$ & $\begin{array}{c}\text { Calcium } \\
\mu \mathrm{g} / \mathrm{g}\end{array}$ & $\begin{array}{c}\text { Potassium } \\
\mu \mathrm{g} / \mathrm{g}\end{array}$ & $\begin{array}{c}\text { Magnesium } \\
\mu \mathrm{g} / \mathrm{g}\end{array}$ & $\begin{array}{c}\text { Sodium } \\
\mu \mathrm{g} / \mathrm{g}\end{array}$ \\
\hline \hline B1NDW3 & 97.3 & $\mathrm{H} 2$ & $1.41 \mathrm{E}+01$ & $1.50 \mathrm{E}+03$ & $5.79 \mathrm{E}+01$ & $1.10 \mathrm{E}+02$ & $3.77 \mathrm{E}+01$ \\
\hline B1NDW3 Dup & 97.3 & $\mathrm{H} 2$ & $1.40 \mathrm{E}+01$ & $1.48 \mathrm{E}+03$ & $5.62 \mathrm{E}+01$ & $1.06 \mathrm{E}+02$ & $3.66 \mathrm{E}+01$ \\
\hline B1NTC9 & 51.8 & $\mathrm{H} 2$ & $1.78 \mathrm{E}+01$ & $1.35 \mathrm{E}+03$ & $8.45 \mathrm{E}+01$ & $1.55 \mathrm{E}+02$ & $5.62 \mathrm{E}+01$ \\
\hline B1NTC9 Dup & 51.8 & $\mathrm{H} 2$ & $1.83 \mathrm{E}+01$ & $1.39 \mathrm{E}+03$ & $8.55 \mathrm{E}+01$ & $1.58 \mathrm{E}+02$ & $5.97 \mathrm{E}+01$ \\
\hline B1NTD1 & 78.8 & $\mathrm{H} 2$ & $1.10 \mathrm{E}+01$ & $1.25 \mathrm{E}+03$ & $8.13 \mathrm{E}+01$ & $1.16 \mathrm{E}+02$ & $3.57 \mathrm{E}+01$ \\
\hline B1NTD1 Dup & 78.8 & $\mathrm{H} 2$ & $1.09 \mathrm{E}+01$ & $1.24 \mathrm{E}+03$ & $7.98 \mathrm{E}+01$ & $1.16 \mathrm{E}+02$ & $3.57 \mathrm{E}+01$ \\
\hline B1NTC7 & 60.8 & $\mathrm{H} 2$ & $1.15 \mathrm{E}+01$ & $1.12 \mathrm{E}+03$ & $9.70 \mathrm{E}+01$ & $1.24 \mathrm{E}+02$ & $4.56 \mathrm{E}+01$ \\
\hline B1NTC7 Dup & 60.8 & $\mathrm{H} 2$ & $1.03 \mathrm{E}+01$ & $1.12 \mathrm{E}+03$ & $9.52 \mathrm{E}+01$ & $1.22 \mathrm{E}+02$ & $4.41 \mathrm{E}+01$ \\
\hline B1NTD3C & 49.8 & $\mathrm{H} 2$ & $1.44 \mathrm{E}+01$ & $1.36 \mathrm{E}+03$ & $8.47 \mathrm{E}+01$ & $1.45 \mathrm{E}+02$ & $3.60 \mathrm{E}+01$ \\
\hline B1NTD3C Dup & 49.8 & $\mathrm{H} 2$ & $1.45 \mathrm{E}+01$ & $1.36 \mathrm{E}+03$ & $8.64 \mathrm{E}+01$ & $1.48 \mathrm{E}+02$ & $3.66 \mathrm{E}+01$ \\
\hline
\end{tabular}

Table 4.38. Cation Exchange Capacity (CEC) of Sediments from the U Farm Direct Push Holes

\begin{tabular}{|l|c|c|c|}
\hline \multicolumn{1}{|c|}{$\begin{array}{c}\text { Sample } \\
\text { ID }\end{array}$} & $\begin{array}{c}\text { Mid Depth } \\
\text { (ft bgs) }\end{array}$ & $\begin{array}{c}\text { Stratigraphic } \\
\text { Unit }\end{array}$ & $\begin{array}{c}\text { CEC } \\
\text { (meq/100g) }\end{array}$ \\
\hline \hline B1NDW3 & 97.3 & $\mathrm{H} 2$ & $8.98 \mathrm{E}+00$ \\
\hline B1NDW3 Dup & 97.3 & $\mathrm{H} 2$ & $8.80 \mathrm{E}+00$ \\
\hline B1NTC9 & 51.8 & $\mathrm{H} 2$ & $8.78 \mathrm{E}+00$ \\
\hline B1NTC9 Dup & 51.8 & $\mathrm{H} 2$ & $9.00 \mathrm{E}+00$ \\
\hline B1NTD1 & 78.8 & $\mathrm{H} 2$ & $7.59 \mathrm{E}+00$ \\
\hline B1NTD1 Dup & 78.8 & $\mathrm{H} 2$ & $7.55 \mathrm{E}+00$ \\
\hline B1NTC7 & 60.8 & $\mathrm{H} 2$ & $7.13 \mathrm{E}+00$ \\
\hline B1NTC7 Dup & 60.8 & $\mathrm{H} 2$ & $7.08 \mathrm{E}+00$ \\
\hline B1NTD3C & 49.8 & $\mathrm{H} 2$ & $8.66 \mathrm{E}+00$ \\
\hline B1NTD3C Dup & 49.8 & $\mathrm{H} 2$ & $8.68 \mathrm{E}+00$ \\
\hline
\end{tabular}

\subsection{Detailed Characterization to Elucidate Controlling Geochemical Processes at the U Tank Farm}

Characterization activities of the direct push samples added some insight as to 1) the processes that control the observed distribution of contaminants and 2) the migration potential of key contaminants in the future. Pore water chemical compositions, calculated by dilution correction of the 1:1 water extracts in the sediment from the direct push samples, were dominated by sodium and bicarbonate for sediments with obvious signs of tank fluids. The most concentrated pore water is shown in Table 5.1 in units of $\mathrm{meq} / \mathrm{L}$. Also included in the table for comparison are the maximum pore water concentrations found in other characterization work previously reported for the T, TX, TY, and SX Tank Farms.

For the U Tank Farm direct push samples, the most saline calculated pore water resided in the $\mathrm{H} 2$ unit and consisted almost entirely of sodium (203 meq/L), with trace amounts of calcium $(0.546 \mathrm{meq} / \mathrm{L})$ and magnesium $(0.125 \mathrm{meq} / \mathrm{L})$. The cation charge for this sample was compensated primarily by bicarbonate $(133 \mathrm{meq} / \mathrm{L})$ and nitrate $(60.9 \mathrm{meq} / \mathrm{L})$, with lesser amounts of sulfate $(6.82 \mathrm{meq} / \mathrm{l})$, fluoride $(5.48 \mathrm{meq} / \mathrm{L})$, chloride $(1.81 \mathrm{meq} / \mathrm{L})$, and chromate $(1.37 \mathrm{meq} / \mathrm{L})$. As shown in Table 4.39 , the most concentrated calculated pore water from the U Tank Farm direct push sampling campaign was less concentrated, and in some cases much less concentrated, than pore waters found in the vadose zone sediments from the SX Tank Farm, but was comparable to those measured in the T, TX, and TY Tank Farms. 
The distribution of the water-extractable major cations in the direct push sediment samples indicates that an ion-exchange process dominates the pore water/sediment interactions where tank fluid has passed by or currently exists. The depth profiles for the divalent alkaline earth cations (calcium, magnesium, and strontium) versus sodium show depleted alkaline earth cation concentrations in the Hanford formation sediments near tank 241-U-110 to a depth of at least $98 \mathrm{ft}$ bgs (the terminal depth of the deepest core sample collected as part of the characterization campaign). Conversely, the water-extractable sodium concentrations in these zones were significantly elevated. These trends suggest that tank fluids that are high in sodium are present at this location.

Table 4.39. Maximum Pore Water Concentrations in Sediments from the Hanford formation Unit (reported in units of $\mathrm{mN}$ )

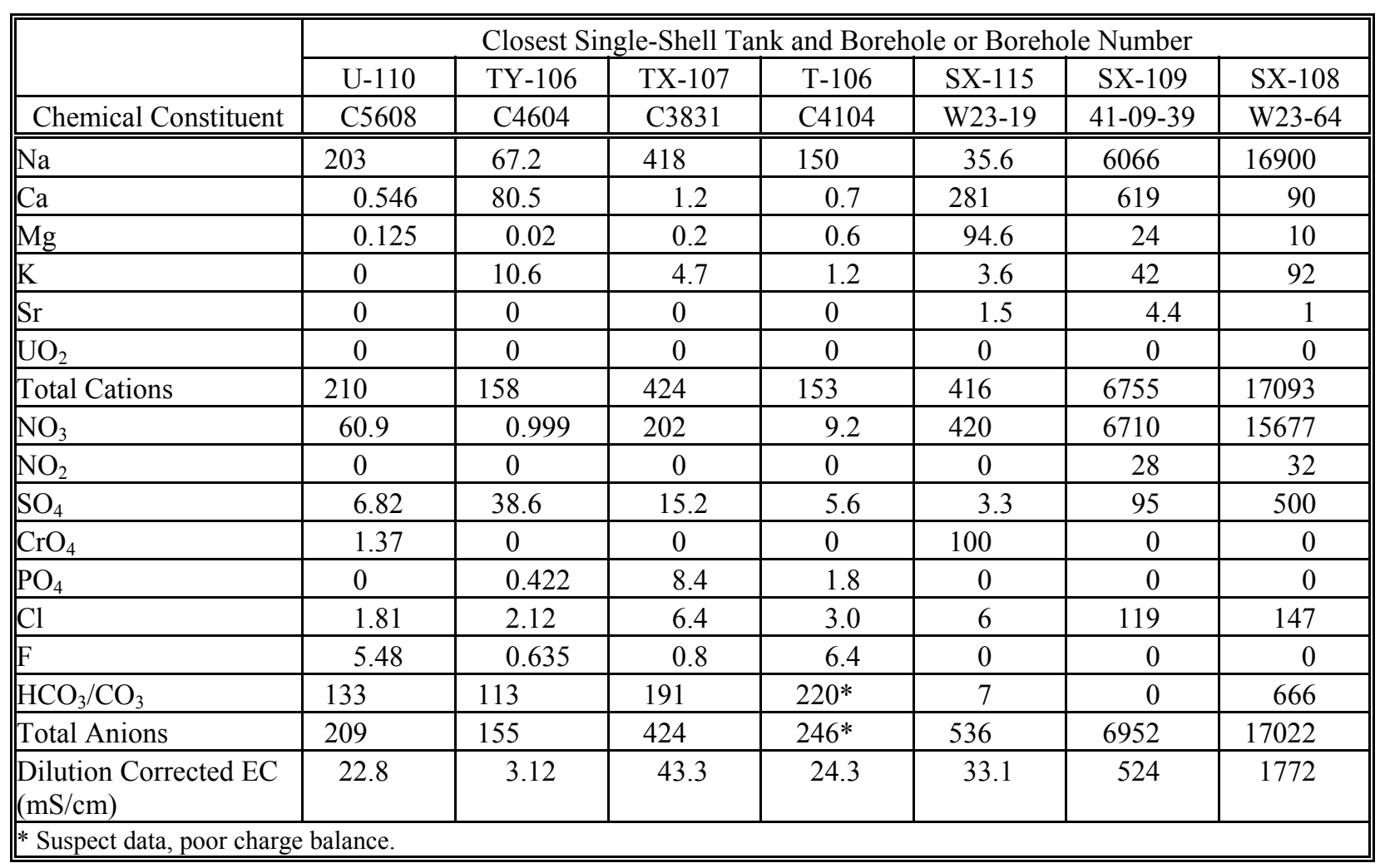

\subsection{Estimates of Contaminant Partition Coefficients}

This section provides the measurements and data synthesis used to provide estimates of the equilibrium partition coefficients of the primary contaminants measured in the $U$ farm direct push holes: uranium, as well as chromium and technetium-99. Site-specific sorption or desorption studies were not specifically performed; however, by combining the data from the dilution-corrected 1:1 water extracts, which represent the pore water, with the concentrations measured in the acid leaches, which are approximate measures of the total leachable concentrations in the sediment, an estimate of the equilibrium partition coefficient $\left(\mathrm{K}_{\mathrm{d}}\right)$ values could be made.

Qualitative estimates of equilibrium $\mathrm{K}_{\mathrm{d}}$ values can be calculated using the inventory estimates (mass or activity per gram of sediment) divided by the estimated pore water concentration of the constituent. These values are found in Tables 4.30 (acid leaches) and 4.27 (pore waters), respectively. Table 4.40 
shows the estimated equilibrium $\mathrm{K}_{\mathrm{d}}$ values for uranium, chromium, and technetium-99 for the U Farm direct push samples. In Table 4.40, the depths where the bulk of the contamination was present are highlighted in red (bold) type and the equilibrium $\mathrm{K}_{\mathrm{d}}$ values that suggest contamination being present at even very low concentrations are highlighted in yellow (darker) shading. The blue shading in Table 4.40 designates data that are more dominated by natural constituents or impacted by low precision analytical values. Generally, where there were significant concentrations of contaminants in the sediments, the $\mathrm{K}_{\mathrm{d}}$ values for uranium were smaller than their values in samples with no obvious signs of tank-related fluids. This is explained by several reasons: 1) the presence of more saline pore waters (competing ions), 2) higher contributions of complexed species (uranyl carbonates) for uranium, which are generally more water leachable, and 3) natural uranium not being as water-extractable as Hanford process uranium. However, the two sediment samples that contained the highest uranium concentrations (B1P3H0B and $\mathrm{B} 1 \mathrm{P} 3 \mathrm{H} 0 \mathrm{~A}$ ) had equilibrium partition coefficients of 37.6 and $8.56 \mathrm{ml} / \mathrm{g}$, respectively. These values were up to two orders of magnitude greater than values measured deeper in push hole C5602. These results indicate that a significant amount of contaminant uranium present in the C5602 samples has become recalcitrant to water leaching. The four samples from direct push hole C5608 that had quantitative $\mathrm{K}_{\mathrm{d}}$ values for technetium-99 were fairly consistent with previously reported $K_{d}$ values for technetium-99 at Hanford (0-0.1 mL/g), with the exception of sample B1PK53B, which had a calculated $\mathrm{K}_{d}$ value of $0.32 \mathrm{ml} / \mathrm{g}$. Sample B1PBB0B, which had a calculated $\mathrm{K}_{\mathrm{d}}$ value of $5.65 \mathrm{ml} / \mathrm{g}$, could likely be impacted by poor analytical resolution, as the reported results for both the water and acid extract were marginally above the sample estimated quantification limits for the respective analyses. Partition coefficient values for chromium in the only samples clearly impacted by tank waste were typical and ranged from 0.82 to $1.82 \mathrm{~mL} / \mathrm{g}$.

Table 4.40. Equilibrium $\mathrm{K}_{\mathrm{d}}$ Values for the U Farm Direct Push Samples

\begin{tabular}{|c|c|c|c|c|c|}
\hline $\begin{array}{c}\text { Sample } \\
\text { Number }\end{array}$ & \begin{tabular}{|c|} 
Borehole \\
Number \\
\end{tabular} & $\begin{array}{l}\text { Mid-Depth } \\
\text { (ft bgs) }\end{array}$ & $\begin{array}{c}\text { Technetium-99 } \mathrm{K}_{\mathrm{d}} \\
(\mathrm{mL} / \mathrm{g})\end{array}$ & $\begin{array}{c}\text { Uranium-238 } \mathrm{K}_{\mathrm{d}} \\
(\mathrm{mL} / \mathrm{g})\end{array}$ & $\begin{array}{c}\text { Chromium } K_{d} \\
(\mathrm{~mL} / \mathrm{g})\end{array}$ \\
\hline B1NDW3B & C5590 & 96.3 & ND & $1.02 \mathrm{E}+02$ & ND \\
\hline B1NDW3A & C5590 & 96.8 & $\mathrm{ND}$ & $1.12 \mathrm{E}+02$ & $4.17 \mathrm{E}+02$ \\
\hline B1NDW3A DUP & C5590 & 96.8 & ND & $1.84 \mathrm{E}+02$ & $6.49 \mathrm{E}+02$ \\
\hline B1NHV0B & C5592 & 62.3 & ND & $6.22 \mathrm{E}+01$ & ND \\
\hline B1NHV0A & C5592 & 62.8 & ND & $3.76 \mathrm{E}+01$ & ND \\
\hline B1NTC6B & C5598 & 50.3 & ND & $3.93 \mathrm{E}+01$ & ND \\
\hline B1NTC6A & C5598 & 50.8 & $\mathrm{ND}$ & $9.21 \mathrm{E}+01$ & ND \\
\hline B1NTC7B & C5598 & 59.8 & ND & $8.60 \mathrm{E}+01$ & ND \\
\hline B1NTC7A & C5598 & 60.3 & ND & $1.22 \mathrm{E}+02$ & ND \\
\hline B1NTC8B & C5598 & 82.3 & $\mathrm{ND}$ & $1.23 \mathrm{E}+02$ & ND \\
\hline B1NTC8B DUP & C5598 & 82.3 & ND & $1.24 \mathrm{E}+02$ & ND \\
\hline B1NTC8A & C5598 & 82.8 & ND & $9.26 \mathrm{E}+01$ & ND \\
\hline B1NTC9B & C5596 & 50.8 & $\mathrm{ND}$ & $2.42 \mathrm{E}+01$ & ND \\
\hline B1NTC9A & C5596 & 51.3 & ND & $2.56 \mathrm{E}+01$ & ND \\
\hline B1NTD0B & C5596 & 61.3 & ND & $6.61 \mathrm{E}+01$ & ND \\
\hline B1NTD0A & C5596 & 61.8 & ND & $6.75 \mathrm{E}+01$ & ND \\
\hline B1NTD1B & C5596 & 77.8 & ND & $7.60 \mathrm{E}+01$ & ND \\
\hline B1NTD1B DUP & C5596 & 77.8 & ND & $5.80 \mathrm{E}+01$ & ND \\
\hline
\end{tabular}




\begin{tabular}{|c|c|c|c|c|c|}
\hline $\begin{array}{c}\text { Sample } \\
\text { Number } \\
\end{array}$ & \begin{tabular}{|c|} 
Borehole \\
Number \\
\end{tabular} & \begin{tabular}{|c} 
Mid-Depth \\
(ft bgs)
\end{tabular} & $\begin{array}{c}\text { Technetium-99 } \mathrm{K}_{\mathrm{d}} \\
(\mathrm{mL} / \mathrm{g})\end{array}$ & $\begin{array}{c}\text { Uranium-238 } K_{d} \\
(\mathrm{~mL} / \mathrm{g})\end{array}$ & $\begin{array}{c}\text { Chromium } K_{\mathrm{d}} \\
(\mathrm{mL} / \mathrm{g})\end{array}$ \\
\hline B1NTD1A & C5596 & 78.3 & ND & $8.84 \mathrm{E}+01$ & $3.14 \mathrm{E}+02$ \\
\hline B1NTD2B & C5596 & 82.8 & ND & $6.63 \mathrm{E}+01$ & ND \\
\hline B1NTD2A & C5596 & 83.3 & ND & $6.87 \mathrm{E}+01$ & ND \\
\hline B1NTD3B & C5600 & 50.3 & ND & $6.54 \mathrm{E}+01$ & ND \\
\hline B1NTD3A & C5600 & 50.8 & ND & $6.61 \mathrm{E}+01$ & ND \\
\hline B1NTD4B & C5600 & 60.3 & ND & $5.60 \mathrm{E}+01$ & $3.45 \mathrm{E}+02$ \\
\hline B1NTD4A & C5600 & 60.8 & ND & $1.13 \mathrm{E}+02$ & $5.44 \mathrm{E}+02$ \\
\hline B1NTD5B & C5600 & 82.3 & ND & $9.50 \mathrm{E}+01$ & $9.99 \mathrm{E}+02$ \\
\hline B1NTD5A & C5600 & 82.8 & ND & $7.62 \mathrm{E}+01$ & $2.49 \mathrm{E}+02$ \\
\hline B1P1K6B & C5600 & 88.8 & ND & $8.02 \mathrm{E}+01$ & ND \\
\hline B1P1K6A & C5600 & 89.3 & ND & $8.15 \mathrm{E}+01$ & ND \\
\hline B1P3F9B & C5604 & 50.8 & ND & $3.07 \mathrm{E}+01$ & ND \\
\hline B1P3F9A & C5604 & 51.3 & ND & $4.85 \mathrm{E}+01$ & ND \\
\hline B1P3H0B & $\mathrm{C} 5602$ & 51.8 & ND & $3.76 \mathrm{E}+01$ & ND \\
\hline B1P3H0A & C5602 & 52.3 & ND & $8.56 \mathrm{E}+00$ & ND \\
\hline B1P3H1B & C5602 & 67.8 & ND & $2.98 \mathrm{E}-01$ & ND \\
\hline B1P3H1A & C5602 & 68.3 & ND & 4.85E-01 & ND \\
\hline B1P3H2B & C5602 & 82.8 & ND & 6.92E-01 & ND \\
\hline $\mathrm{B} 1 \mathrm{P} 3 \mathrm{H} 2 \mathrm{~A}$ & C5602 & 83.3 & ND & ND & ND \\
\hline B1PBB0B & C5602 & 91.8 & $5.65 \mathrm{E}+00$ & $1.09 \mathrm{E}+02$ & ND \\
\hline B1PBB0A & C5602 & 92.3 & ND & $1.29 \mathrm{E}+02$ & ND \\
\hline B1PBB1B & C5606 & 51.8 & ND & $1.94 \mathrm{E}+01$ & ND \\
\hline B1PBB1A & C5606 & 52.3 & ND & $1.06 \mathrm{E}+01$ & ND \\
\hline B1PBB1A DUP & $\mathrm{C} 5606$ & 52.3 & ND & $1.03 \mathrm{E}+01$ & ND \\
\hline B1PK51B & C5608 & 64.3 & ND & $2.26 \mathrm{E}+01$ & $5.42 \mathrm{E}+01$ \\
\hline B1PK51A & C5608 & 64.8 & ND & $9.01 \mathrm{E}+00$ & $3.02 \mathrm{E}+02$ \\
\hline B1PK52B & $\mathrm{C} 5608$ & 85.8 & 2.24E-02 & $7.72 \mathrm{E}+01$ & $1.62 \mathrm{E}+00$ \\
\hline B1PK52A & $\mathrm{C} 5608$ & 86.3 & $1.78 \mathrm{E}-02$ & $2.29 \mathrm{E}+01$ & $1.32 \mathrm{E}+00$ \\
\hline B1PK53B & C5608 & 97.8 & $3.20 \mathrm{E}-01$ & $5.06 \mathrm{E}-01$ & $1.82 \mathrm{E}+00$ \\
\hline B1PK53A & C5608 & 98.3 & 6.34E-02 & 3.82E-01 & 8.15E-01 \\
\hline \multicolumn{6}{|c|}{$\begin{array}{l}\text { ND indicates the calculation could not be made because data was less than sample-estimated quantification limits. } \\
\text { Faint blue (light) shading data are likely more dominated by natural constituents. } \\
\text { Red (bold) type signifies depths where sediments show obvious signs of some tank-related fluids. } \\
\text { Yellow (dark) shading signifies } \mathrm{K}_{\mathrm{d}} \text { values that are dominated by tank fluids. }\end{array}$} \\
\hline
\end{tabular}




\subsection{Summary and Observations}

In this section, a summary of information about the characterization of the U Tank Farm direct push sediments is presented. Interpretation of the data has been included to aid in making decisions on what interim actions and future studies are needed to make sound remediation decisions at the U Tank Farm.

\subsection{U Tank Farm Physical Geology Model}

Assessment of data from nearby boreholes coupled with analysis of material recovered from the direct push holes has led to the interpretation that the deposits beneath the U Tank Farm consist predominantly of the gravel-dominated Hanford formation H1 unit and sand-dominated Hanford formation $\mathrm{H} 2$ unit. Based on the downhole total gamma and neutron moisture logs, the contact between the $\mathrm{H} 1$ and $\mathrm{H} 2$ units of the Hanford formation appears to lie between 50 and $54 \mathrm{ft}$. These facies were deposited onto the giant Cold Creek bar during repeated Pleistocene cataclysmic floods. Beneath the Hanford formation is the Cold Creek unit, which consists of an upper fine sand to silt unit and a lower unit of variably cemented caliche, representing a buried paleosol sequence. Combined, the Cold Creek unit may be up to $40 \mathrm{ft}$ thick and its upper surface has a slight regional dip to the southwest. Below the Cold Creek unit is a thick sequence of variably cemented Ringold fluvial gravel (Rwi).

The hydrogeology of the area surrounding the U Tank Farms are complicated by the presence of clastic dikes that cross-cut many of the primary sedimentary layers. Clastic dikes form as the result of compaction of water-rich sediments during rapid burial. Clastic dikes are important from a hydrogeology standpoint as infiltrating solutions will preferentially flow along these vertical to sub-vertical conduits of higher conductivity. Therefore, horizontal layers that would otherwise serve as capillary barriers would be circumvented.

\subsection{U Tank Farm Characterization Activities and Data}

The next several sections summarize geochemical and physical characterization data collected on sediment from the direct push holes emplaced within the U Tank Farm. These characterization activities emphasized tests that provided basic characterization data and were fundamental to determining the distribution of mobile contaminants in the vadose zone sediments. Such information on the direct push sediments included moisture content, total and inorganic carbon content, $\mathrm{pH}$, electrical conductivity (EC), and measurements of major cations, anions, and trace metals (including technetium-99 and uranium-238) in 1:1 sediment:water and $8 \mathrm{M}$ nitric acid extracts. Gamma energy analysis (GEA) of the sediments was also performed to search for any detectable anthropogenic gamma-emitting radionuclides.

\subsubsection{Sampling Summary at the U Tank Farm}

A geochemical investigation in the vicinity of tanks 241-U-101, 241-U-104/241-U-105, 241-U-110, 241-U-112, and 241-U-201 was performed using pairs of direct push probe holes. A total of 20 directpush holes were driven within the U Tank Farm; ten of these holes were logged for moisture, gross gamma, and in some cases spectral gamma using calibrated probes and ten were driven for the purpose of retrieving vadose zone sediment for characterization and analysis. The locations of the direct push holes were chosen to investigate an estimated 50,000 gallon leak of bismuth phosphate metals waste from tank 
241-U-104, small leaks of REDOX supernatant from tanks 241-U-110 and 241-U-112, a 30,000 gallon leak of waste from tank 241-U-101 (as yet unconfirmed from measurements in the vadose zone), and a resistivity anomaly near the U Farm 200 series tanks.

\subsubsection{Moisture Content}

The depths at which the direct push samples were collected were based on neutron-moisture measurements performed in the field. The intent was to retrieve vadose zone sediment from regions of elevated moisture. Of the ten direct push holes emplaced during the U Tank Farm campaign, five of them contained very moist sediment. Specifically, sediments from at least one liner sample retrieved from push holes C5598, C5600, C5602, C5606, and C5608 had moisture contents in excess of $15 \mathrm{wt} \%$. The highest sediment moisture content measured in the U Farm direct push samples, at 19.8\% (C5602), was consistent with the peak moisture content (18.3\%) measured in the Hanford formation H2 unit in the background borehole (C3393). Therefore, no correlation can be made between moisture content and the potential presence of tank waste in the sediments (i.e., high moisture contents are not caused by the presence of waste fluids).

\subsubsection{Contamination Profile around tanks 241-TY-105 and 241-TY-106}

Several parameters, including high $\mathrm{pH}$ and electrical conductivity values, as well as high concentrations of nitrate, technetium-99, sodium, and uranium in water and acid extracts were used as indicators to determine the subsurface regions impacted by potential tank leaks in the U Tank Farm. Contamination of the sediments with anthropogenic radionuclide elements was confirmed using direct GEA measurements of the samples. The following paragraphs present the highlights from these tests.

The first parameter measured was the $\mathrm{pH}$ of water extracts of the vadose zone sediment. Based on the assumption that tank-related waste fluids are generally caustic and often very caustic $(>1 \mathrm{M}$ free hydroxide), elevated $\mathrm{pH}$ profiles should be indicative of the near-field region close to the source where the caustic fluid entered the sediments. Nearly all of the extract samples tested had $\mathrm{pH}$ values in the normal range for Hanford formation sediments (between 7.5 and 8.0). However, one of the push holes, C5608, contained sediment with an elevated $\mathrm{pH}$. Specifically, all six of the cores analyzed from push hole $\mathrm{C} 5608$ had soil $\mathrm{pH}$ values in excess of 9.0. The peak soil $\mathrm{pH}$, at 10.3, was measured in a sample retrieved from approximately $86 \mathrm{ft}$ bgs (B1PK52A). Therefore, it appears that direct push hole C5608, emplaced near tank 241-U-110, was located in close proximity to the location of the leak.

The second parameter that was assessed to investigate proposed tank leaks was the dilution-corrected water extract electrical conductivity (EC) of the sediment samples. The pore water-corrected EC data for all of the samples from the U Tank Farm (except those from push hole C5608) were low, with a range of 1.25 to $6.39 \mathrm{mS} / \mathrm{cm}$. Conversely, samples collected from push hole $\mathrm{C} 5608$ had porewater-corrected conductivities ranging from 5.13 to $22.8 \mathrm{mS} / \mathrm{cm}$. The peak porewater-corrected conductivity $(22.8$ $\mathrm{mS} / \mathrm{cm}$ ) was measured in the deepest sample analyzed from push hole C5608. For comparison, the background borehole (C3393) contained samples with porewater-corrected conductivities ranging from 0.978 to $10.5 \mathrm{mS} / \mathrm{cm}$. Therefore, with the exception of samples from push hole C5608, the U Tank Farm direct push samples appeared to be dilute with respect to dissolved salt content and were comparable to dissolved salt loads measured in samples from the background borehole. Based on this, it appears that 1) there is little indication of residual tank waste in the sediments analyzed as part of this study based on 
elevated dissolved salts and 2) sufficient recharge has likely occurred to drive the bulk of the contamination deeper into the vadose zone.

The third parameter that was used to investigate the extent of tank waste-related contamination in the vadose zone was sodium concentrations. The only samples that contained sodium concentrations well above normal background levels were those from push hole C5608. All six of the liner samples analyzed from push hole C5608 contained nearly an order of magnitude or higher water-extractable sodium concentrations than those measured from borehole C3393 (the background borehole). Coincident with the elevated sodium, water-extractable concentrations of calcium, potassium, and magnesium were negligible to non-quantifiable in these samples. It is apparent for these samples that sodium has driven the divalent cations off the exchange sites. Based on these data, it is clear that a sodium-rich waste stream has migrated to at least $96 \mathrm{ft}$ bgs adjacent to tank 241-U-110. However, the total vertical extent of the ion exchange front is unknown due to the lack of sediment samples from deeper in the vadose zone.

Sodium was present as the dominant cation (or co-dominant cation) in all of the samples analyzed where contamination was suspected. Sodium was also present as the dominant water-extractable cation in most of the samples from the background borehole. The lack of quantifiable calcium in the samples indicates that the sediments in this region have been impacted by a sodium-bearing waste fluid. The source(s) appears to be a moderately concentrated sodium-bearing waste solution that has displaced the natural divalent cations from the cation exchange sites in the sediments. The total vertical (and lateral) extent of the ion exchange front is unknown due to the lack of sediment samples from deeper in the vadose zone.

Concentrations of mobile constituents, such as water-extractable uranium, technetium-99, and nitrate, are three additional parameters that can be used to define the extent of subsurface contamination. Technetium-99 and nitrate are both considered highly mobile in the subsurface; therefore, their presence in samples can typially be used to estimate the total extent of contaminant plume migration. Of the ten push holes analyzed as part of this study, two contained sediment with quantifiable activities of technetium-99. Sediment from one of the two push holes (C5602) only contained a trace amount of technetium-99 $(<0.2 \mathrm{pCi} / \mathrm{g})$, while samples analyzed from push hole $\mathrm{C} 5608$, which was emplaced adjacent to tank 241-U-110, contained appreciable activities of technetium-99. The technetium-99 measured in push hole $\mathrm{C} 5608$ ranged from 0.054 to $48.8 \mathrm{pCi} / \mathrm{g}$. Because technetium- 99 was still detected in the deepest samples collected from push hole C5608, the maximum vertical extent of mobile contaminants at this location can not be ascertained. Similar to the technetium-99 results, the only samples that were significantly elevated in dissolved anions were those collected from push hole C5608. Specifically, the two deepest sample strings collected from push hole C5608 (85 ft bgs and $98 \mathrm{ft} \mathrm{bgs,}$ respectively) contained elevated water-extractable nitrate (as well as fluoride and phosphate). The peak water-extractable nitrate $(578 \mu \mathrm{g} / \mathrm{g})$ measured in the C5608 push hole was nearly two orders of magnitude greater than that measured in the background borehole C3393 $(8.54 \mu \mathrm{g} / \mathrm{g})$.

Naturally occurring uranium is present in a crystalline form that is very recalcitrant to leaching. Therefore, elevated amounts of uranium in the 1:1 sediment:water extracts are typically indicative of contaminant uranium. Concentrations of water-leachable uranium-238 were significantly elevated with respect to background in only samples from push hole C5602, which was emplaced just southeast of tank 241-U-105. While the background borehole sediments had water-extractable uranium concentrations ranging from $1.93 \mathrm{E}-04$ to $1.79 \mathrm{E}-03 \mu \mathrm{g} / \mathrm{g}$, sediments retrieved from push hole C5602 contained as much 
as $5.16 \mu \mathrm{g} / \mathrm{g}$ water-extractable uranium. It does not appear that all of the samples retrieved from push hole C5602 contained contaminant uranium. Water extract data for the two deepest samples analyzed appeared to be representative of natural uranium. Sediments retrieved from push holes C5606 and C5608, emplaced near tank 241-U-112 and 241-U-110, respectively, could contain small amounts of contaminant uranium (based on the water-extract data), but at $0.13 \mu \mathrm{g} / \mathrm{g}$ or less uranium, the concentrations are not significantly elevated above background concentrations.

Slightly elevated concentrations of acid-extractable uranium-238 $(1.35 \mu \mathrm{g} / \mathrm{g})$ were found in samples from push hole C5606, but significantly elevated concentrations (as much as $731 \mu \mathrm{g} / \mathrm{g}$ ) were measured in extracts of sediments from push hole C5602. Push hole C5602 was emplaced near tank 241-U-104, which was estimated to have released more than $5100 \mathrm{~kg}$ of uranium to the vadose zone. Additionally, previous logging of drywells in the area has identified significant uranium contamination in the vadose zone near tank 241-U-104; therefore, the observation of more than $700 \mu \mathrm{g} / \mathrm{g}$ uranium in sediment from push hole C5602 was not unexpected.

The final indicator used to define the presence of tank-related waste in these samples was direct measurement of sediments for gamma-emitting radionuclides. GEA of the sediment samples showed that natural potassium-40 was present in sediments collected from all of the direct push holes. Additionally, contaminant uranium was found in two samples retrieved from push hole C5602.

\subsubsection{Sources of Contamination in the U Tank Farm}

After evaluating all the characterization and analytical data, there is no question that the vadose zone in the vicinity of tanks 241-U-104 and 241-U-105 has been contaminated by tank-related waste. This observation is not new, as gamma logging of drywells in the area has identified uranium contamination at the same depths interrogated by push hole C5602. Given that the deepest sample string analyzed from push hole C5602 contained trace activities of technetium-99, it is obvious that tank waste contamination has impacted the vadose zone to at least a depth of $92 \mathrm{ft}$ bgs at this location. However, the scope of the sampling campaign was to acquire additional samples to better understand the aerial extent of contamination in the U Tank Farm; therefore, future characterization activities (i.e., a borehole) will be required to understand the total vertical depth of contamination at this location.

The vadose zone south tank $241-\mathrm{U}-110$ has also been affected by a tank-related waste solution. The presence of sodium as the dominant water-extractable cation indicates that a high sodium-bearing waste stream has created a cation exchange front in this region that has pushed the typical divalent cations (calcium and magnesium) off the surface exchange sites. The presence of significantly elevated concentrations of technetium-99 and nitrate in the deepest samples collected indicate that the vadose zone has been impacted to at least a depth of $98 \mathrm{ft}$ bgs. The high soil $\mathrm{pH}$, coupled with the presence of mobile contaminants deep in the vadose zone, makes it clear that a release from tank 241-U-110 is the source of contamination intercepted by push hole C5608. Again, the total vertical extent of contamination at this location can not be derived from the direct push sampling results.

Of the remaining direct push samples analyzed, only two contained quantifiable or elevated concentrations of mobile tank waste contaminants. Samples from push hole C5600, which was emplaced southwest of tank 241-U-105 contained a quantifiable activity of technetium-99 in the acid extract of the sample collected from $88 \mathrm{ft}$ bgs. Interestingly, this result was not corroborated by the water extract results, which failed to detect technetium-99 at a concentration above the sample estimated limit of 
quantification. This sole data point should not be dismissed as an outlier, and it is clear that additional data should be collected prior to assessing and confirming the level of contamination southwest of tank 241-U-105.

An interesting observation afforded by the acid extract data is that elevated concentrations of technetium-99 tend to correlate with unusually high concentrations of chrome. High concentrations of technetium-99 do not correlate with uranium-238, but this is expected because partition coefficients for $\mathrm{U}$ and Tc are quite different in these sediments. Partition coefficients for chrome and technetium also predict that the two elements should be fractionated, so it is enigmatic that we find $\mathrm{Tc}$ and $\mathrm{Cr}$ together in the same samples. This unusual association may be completely fortuitous, but the correlation may be indicative of a co-precipitation mechanism. A co-precipitation mechanism is not unrealistic, because the ionic radii of $\mathrm{Fe}(\mathrm{III}), \mathrm{Cr}(\mathrm{III})$, and $\mathrm{Tc}(\mathrm{IV})$ are approximately the same (PAULING, 1947). However, a great deal of fundamental mineralogical investigation is necessary to determine if this hypothesis deserves closer scrutiny

Two samples collected from push hole C5606 (emplaced northeast of tank 241-U-112) contained slightly elevated concentrations of acid-extractable uranium. Tank 241-U-112 was estimated to have released only $24 \mathrm{~kg}$ of uranium to the vadose; therefore, the lack of significant uranium contamination in a push hole emplaced near the tank is not an unreasonable finding. As with the data collected southwest of tank 241-U-105, these data points should not be considered conclusive evidence of the presence of tank waste in the vadose zone near tank 241-U-112.

Aside from elevated concentrations of sodium in most water and several acid extracts, no other tank waste constituents were observed at elevated concentrations in the push holes emplaced northeast of tank 241-U-101 or north of the 200 series tanks. Additionally, the inferred porewater chemistry, based on water extracts of the samples collected from push hole C5604, do not support the resistivity anomaly observed by Rucker et al (2006) north of the 200 series tanks.

\subsection{Controlling Geochemical Processes at the U Tank Farm}

Characterization activities showed that pore water chemical compositions, calculated by dilution correction of the 1:1 water extracts in the sediment from the direct push samples, were dominated by sodium and bicarbonate for sediments with obvious signs of tank fluids. The distribution of the waterextractable major cations in the direct push sediment samples indicates that an ion-exchange process dominates the pore water/sediment interactions where tank fluid has passed by or currently exists. The depth profiles for the divalent alkaline earth cations (calcium, magnesium, and strontium) versus sodium show depleted alkaline earth cation concentrations in the Hanford formation sediments near tank 241-U110 to a depth of at least $98 \mathrm{ft}$ bgs (the terminal depth of the deepest core sample collected as part of the characterization campaign). Conversely, the water-extractable sodium concentrations in these zones were significantly elevated. These trends suggest that tank fluids that are high in sodium are present at this location.

\subsection{Estimates of Contaminant Partition Coefficients}

Qualitative estimates of equilibrium $\mathrm{K}_{\mathrm{d}}$ values showed that where there were significant concentrations of contaminants in the sediments, the $\mathrm{K}_{d}$ values for uranium were smaller than their values 
in samples with no obvious signs of tank-related fluids. This is explained by several reasons: 1) the presence of more saline pore waters (competing ions), 2) higher contributions of complexed species (uranyl carbonates) for uranium, which are generally more water leachable, and 3) natural uranium not being as water-extractable as Hanford process uranium. However, the two sediment samples that contained the highest uranium concentrations (B1P3H0B and $\mathrm{B} 1 \mathrm{P} 3 \mathrm{H} 0 \mathrm{~A})$ had equilibrium partition coefficients of 37.6 and $8.56 \mathrm{ml} / \mathrm{g}$, respectively, indicating that a significant amount of contaminant uranium present in the samples has become recalcitrant to water leaching. Samples that had quantitative $\mathrm{K}_{\mathrm{d}}$ values for technetium-99 were fairly consistent with previously reported $\mathrm{K}_{\mathrm{d}}$ values for technetium-99 at Hanford $(0-0.1 \mathrm{~mL} / \mathrm{g})$, and partition coefficient values for chromium were typical and ranged from 0.82 to $1.82 \mathrm{~mL} / \mathrm{g}$. 


\subsection{References}

American Society for Testing and Materials (ASTM) D2216-98. 1998. Test Method for Laboratory Determination of Water (Moisture) Content of Soil and Rock by Mass. American Society for Testing and Materials, West Conshohocken, Pennsylvania.

American Society for Testing and Materials (ASTM) D2488-93. 1993. Standard Practice for Description and Identification of Soils (Visual-Manual Procedure). American Society for Testing and Materials, West Conshohocken, Pennsylvania.

American Society for Testing and Materials (ASTM) E1915-01. 2001. Standard Test Methods for Analysis of Metal Bearing Ores and Related Materials by Combustion Infrared Absorption Spectrometry. American Society for Testing and Materials, West Conshohocken, Pennsylvania.

American Society for Testing and Materials (ASTM) D5298-94. 2002. Test Method for Measurement of Soil Potential (Suction) Using Filter Paper. American Society for Testing and Materials, West Conshohocken, Pennsylvania.

American Society of Agronomy (ASA). 1986a. "Hydrometer Method." Chapter 15-5 in "Methods of Soil Analysis-Part 1", 2nd edition of Physical and Mineralogical Methods, SSSA Book Series No. 5, ed. A Klute, pp. 404-408. Soil Science Society of America, Madison, Wisconsin.

American Society of Agronomy (ASA). 1986b. "Pynchnometer Method." Chapter 14-3 in Methods of Soil Analysis-Part 1, 2nd edition of Physical and Mineralogical Methods, SSSA Book Series No. 5, ed. A Klute, pp. 378-379. Soil Science Society of America, Madison, Wisconsin.

American Society of Agronomy (ASA). 1996. Methods of Soil Analysis-Part 3, Chemical Methods, SSSA Book Series 5, ed. DL Sparks, Soil Science Society of America, Madison, Wisconsin.

Baker, V. R., B. N. Bjornstad, A. J. Busacca, K. R. Fecht, E. P. Kiver, U. L. Moody, J. G. Rigby, D. F. Stradling, and A. M. Tallman. 1991. "Quaternary Geology of the Columbia Plateau." Quaternary Nonglacial Geology Conterminous U.S., Vol. K-2, pp. 215-250, R. B. Morrison (ed.), Geological Society of America, Boulder, Colorado.

Bjornstad BN. 1984. Suprabasalt Stratigraphy within and adjacent to the Reference Repository Location: SD-BWI-DP-039. Rockwell Hanford Operations, Richland, Washington.

Bjornstad BN. 1990. Geohydrology of the 218-W-5 Burial Ground, 200 West Area, Hanford Site. PNL-7336, Pacific Northwest Laboratory, Richland, Washington.

Bjornstad, B.N., K.R. Fecht, and C.J. Pluhar. 2001. Long History of Pre-Wisconsin, Ice Age Cataclysmic Floods: Evidence from Southeastern Washington State. Journal of Geology, Vol. 109, p. 695-713.

Bjornstad BN. 2006. On the Trail of the Ice Age Floods: A Geological Field Guide to the Mid-Columbia Basin, Keokee Co. Publishing, Inc., Sandpoint, Idaho.

Brown, D.J. 1959. Subsurface Geology of the Hanford Separation Areas. HW-61780, General Electric Company, Richland, Washington.

Brown DJ. 1960. An Eolian Deposit Beneath 200-West Area. HW-67549, General Electric Company, Richland, Washington. 
Crumpler JD. 2003. Site Specific SST Phase 1 RFI/CMS Work Plan Addendum for WMAs C, A-AX and U. RPP-16608. CH2M HILL Hanford Group, Inc., Richland, Washington.

Deka RN, M Wairiu, PW Mtakwa, CE Mullins, EM Veenendaal, and J Towend. 1995. "Use and Accuracy of the Filter Paper Method for Measuring Soil Matric Potential.” European J. of Soil Sci. 46:233-238.

DOE (US Department of Energy). 1988. Consultation Draft Site Characterization Plan. DOE/RL-0164, 9 volumes, U.S. Department of Energy Richland Operations Office, Richland, Washington.

DOE (US Department of Energy). 1999. Phase 1 RCRA Facility Investigation/Corrective Measures Study Work Plan for the SST Waste Management Areas. DOE/RL-99-36, Rev. 0, U.S. Department of Energy, Richland Operations Office, Richland, Washington.

DOE (US Department of Energy). 2002. Standardized Stratigraphic Nomenclature for Post-RingoldFormation Sediments Within the Pasco Basin. DOE/RL-2002-39, U.S. Department of Energy, Richland Operations Office, Richland, Washington.

DOE-GJO. 1997. U Tank Farm Report, Vadose Zone Characterization Project. GJO-97-1-TAR, prepared for the U.S. Department of Energy, Richland Operations Office, Richland, Washington.

EPA Method 300.0A. 1984. Test Method for the Determination of Inorganic Anions in Water by Ion Chromatography. EPA-600/4-84-017, U.S. Environmental Protection Agency, Washington, D.C.

EPA Method 3050B. 2000a. "Acid Digestion of Sediments, Sludges, and Soils." Test Methods for Evaluating Solid Waste, Physical/Chemical Methods. EPA Publication SW-846, [Online manual]. Available URL: http://www.epa.gov/epaoswer/hazwaste/test/sw846.htm

EPA Method 6010B. 2000b. "Inductively Coupled Plasma-Atomic Emission Spectrometry." Test Methods for Evaluating Solid Waste, Physical/Chemical Methods. EPA Publication SW-846, [Online manual]. Available URL: http://www.epa.gov/epaoswer/hazwaste/test/sw846.htm

EPA Method 6020. 2000c. "Inductively Coupled Plasma-Mass Spectrometry." Test Methods for Evaluating Solid Waste, Physical/Chemical Methods. EPA Publication SW-846, [Online manual]. Available URL: http://www.epa.gov/epaoswer/hazwaste/test/sw846.htm

Fecht KR, BN Bjornstad, DG Horton, GV Last, SP Reidel, and KA Lindsey. 1999. Clastic injection dikes of the Pasco Basin and vicinity: geologic atlas series. BHI-01103, Rev. 0, Bechtel Hanford Company, Richland, Washington.

Folk RL. 1968. Petrology of Sedimentary Rocks. Hemphill, Austin, Texas.

Fredrickson JK, JM Zachara, DW Kennedy, RK Kukkadapu, JP McKinley, SM Heald, C Liu, and AE Plymale. 2004. Reduction of $\mathrm{TcO}^{-4}$ by sediment-associated biogenic Fe(II). Geochimica et Cosmochimica Acta 68, 3171-3187.

Gee GW, MJ Fayer, ML Rockhold, and MD Campbell. 1992. "Variations in Recharge at the Hanford Site," NW Sci. 66:237-250.

Gee GW. 1987. Recharge at the Hanford Site. Status Report. PNL-6403, Pacific Northwest Laboratory, Richland, Washington. 
Hodges, F.N. and C.J. Chou. 2000. Groundwater Quality Assessment for Waste Management Area U: First Determination. PNNL-13282, Pacific Northwest National Laboratory, Richland, Washington.

Johnson VG, TE Jones, SP Reidel, and MI Wood. 1999. Subsurface Physical Conditions Description of the S-SX Waste Management Area. HNF-4936, Rev. 0, Lockheed Martin Hanford Corporation, Richland, Washington.

Last GV, BN Bjornstad, MP Bergeron, DW Wallace, DR Newcomer, JA Schramke, MA Chamness, CS Cline, SP Airhart, and JS Wilbur. 1989. Hydrogeology for the 200 Areas Low-Level Burial Grounds - An Interim Report. PNL-6820, Pacific Northwest Laboratory, Richland, Washington.

Lindenmeier CW, RJ Serne, BN Bjornstad, GW Gee, HT Schaef, DC Lanigan, MJ Lindberg, RE Clayton, VL LeGore, IV Kutnyakov, SR Baum, KN Geiszler, KMM Valenta, TS Vickerman, and LJ Royack. 2002. Characterization of Vadose Zone Sediment: RCRA Borehole 299 E33 338 Located Near the B-BX-BY Waste Management Area, PNNL 14121, Pacific Northwest National Laboratory, Richland, Washington.

Lindsey KA, MP Connelly, and BN Bjornstad. 1992. Geologic Setting of the 200 West Area - An Update. WHC-SD-EN-TI-008, Westinghouse Hanford Company, Richland, Washington.

Lindsey, K.A. 1995. Miocene-to Pliocene-Aged Suprabasalt Sediments of the Hanford Site, SouthCentral Washington. BHI-00184, Bechtel Hanford Incorporated, Richland, Washington.

Lindsey KA, KD Reynolds, and SE Kos. 2001a. Vadose Zone Geology of Boreholes 299-E33-45 and 299-E33-46 B-BX-BY Waste Management Area Hanford Site, South-Central Washington. RPP8681, Rev. 0, CH2M HILL Hanford Group, Inc., Richland, Washington.

Lindsey KA, SE Kos, and KD Reynolds. 2001b. Vadose Zone Geology of Boreholes 299-W10-27 and 299-W11-39 T-TX-TY Waste Management Area Hanford Site, South-Central Washington. RPP8531, Rev. 0, CH2M HILL Hanford Group, Inc., Richland, Washington.

Or D and JM Wraith. 2002. "Soil Water Content and Water Potential Relationships." In Soil Physics Companion, ed. AW Warrick, pp. 49-84, CRC Press, Boca Raton, Florida.

Pacific Northwest Laboratory (PNL). 1990. Procedures for Groundwater Investigations. PNL MA-567, Pacific Northwest Laboratory, Richland, Washington.

Pacific Northwest National Laboratory (PNNL). 1997. Gamma Energy Analysis Operation and Instrument Verification Using the Genie2000 ${ }^{T M}$ Support Software. PNNL-RRL-01, Pacific Northwest National Laboratory, Richland, Washington.

Pacific Northwest National Laboratory (PNNL). 1998. Inductively Coupled Plasma Mass Spectrometric (ICP-MS) Analysis. PNNL-AGG-415, Pacific Northwest National Laboratory, Richland, Washington.

Pacific Northwest National Laboratory (PNNL). 2000. Liquid Scintillation Counting and Instrument Verification Using the 1400 DSM ${ }^{\mathrm{TM}}$ Support Software. PNNL-AGG-002, Pacific Northwest National Laboratory, Richland, Washington. 
Pacific Northwest National Laboratory (PNNL). 2005. Soil Water Content. PNNL-AGG-WC-001 Rev.0, Pacific Northwest National Laboratory, Richland, Washington.

Pauling, L. 1947. Atomic radii and interatomic distances in metals. Journal of American Chemical Society 69, 542-553.

Pluhar CJ, BN Bjornstad, SP Reidel, RS Coe, and PB Nelson. 2006. Magnetostratigraphic evidence from the Cold Creek bar for onset of ice-age cataclysmic floods in eastern Washington during the Early Pleistocene. Quaternary Research, v. 65, p. 123-135.

Price WH and KR Fecht. 1976. Geology of the 241-BX Tank Farm. ARH-LD-130, Atlantic Richfield Hanford Company, Richland, Washington.

Randall R and R Price. 2007. Small Diameter Geophysical Logging in the 241-U Tank Farm. Pacific Northwest Geophysics/Three Rivers Scientific, Richland Washington.

Reidel SP and KR Fecht. 1994. Geologic Map of the Priest Rapids 1:100,000 Quadrangle, Washington. Open-File Report 94-13, Washington Division of Geology and Earth Resources.

Reidel SP and MA Chamness. 2007. Geology Data Package for the Single-Shell Tank Waste Management Areas at the Hanford Site. PNNL-15955, Pacific Northwest National Laboratory, Richland, Washington.

Rhoades JD. 1996. "Salinity: Electrical Conductivity and Total Dissolved Solids." In Methods of Soil Analysis Part 3, ed. JM Bigham, pp. 417-435. American Society of Agronomy, Madison, Wisconsin.

Rucker D, M Levitt, C Henderson, and K Williams. 2006. Surface Geophysical Exploration of U Farm at the Hanford Site. RPP-RPT-31557, Rev. 0, CH2M HILL Hanford, Group, Richland, Washington.

Serne RJ, HT Schaef, BN Bjornstad, BA Williams, DC Lanigan, DG Horton, RE Clayton, VL LeGore, MJ O’Hara, CF Brown, KE Parker, IV Kutnyakov, JN Serne, AV Mitroshkov, GV Last, SC Smith, CW Lindenmeier, JM Zachara, and DS Burke. 2002a. Characterization of Vadose Zone Sediment: Uncontaminated RCRA Borehole Core Samples and Composite Samples. PNNL-13757-1, Pacific Northwest National Laboratory, Richland, Washington.

Serne RJ, HT Schaef, BN Bjornstad, DC Lanigan, GW Gee, CW Lindenmeier, RE Clayton, VL LeGore, MJ O'Hara, CF Brown, RD Orr, G.V Last, IV Kutnyakov, DS Burke, TC Wilson, and BA Williams. 2002b. Characterization of Vadose Zone Sediment: Borehole 299-W23-19 [SX-115] in the S-SX Waste Management Area. PNNL-13757-2, Pacific Northwest National Laboratory, Richland, Washington.

Serne RJ, GV Last, HT Schaef, DC Lanigan, CW Lindenmeier, CC Ainsworth, RE Clayton, VL LeGore, MJ O'Hara, CF Brown, RD Orr, IV Kutnyakov, TC Wilson, KB Wagnon, BA Williams, and DB Burke. 2002c. Characterization of Vadose Zone Sediment, Part 4: Slant Borehole SX-108 in the S-SX Waste Management Area. PNNL-13757-4, Pacific Northwest National Laboratory, Richland, Washington. 
Serne RJ, GV Last, GW Gee, HT Schaef, DC Lanigan, CW Lindenmeier, RE Clayton, VL LeGore, RD Orr, MJ O'Hara, CF Brown, DS Burke, AT Owen, IV Kutnyakov, and TC Wilson. 2002d. Characterization of Vadose Zone Sediment: Borehole 41-09-39 in the S-SX Waste Management Area. PNNL-13757-3, Pacific Northwest National Laboratory, Richland, Washington.

Serne RJ, GV Last, GW Gee, HT Schaef, DC Lanigan, CW Lindenmeier, MJ Lindberg, RE Clayton, VL LeGore, RD Orr, IV Kutnyakov, SR Baum, KN Geiszler, CF Brown, MM Valenta, and TS Vickerman. 2002e. Characterization of Vadose Zone Sediment: Borehole 299-E33-45 Near BX102 in the B-BX-BY Waste Management Area. PNNL-14083, Pacific Northwest National Laboratory, Richland, Washington.

Serne RJ, BN Bjornstad, GW Gee, HT Schaef, DC Lanigan, CW Lindenmeier, RD Orr, VL LeGore, RE Clayton, MJ Lindberg, IV Kutnyakov, SR Baum, KN Geiszler, MM Valenta, TS Vickerman, and LJ Royack. 2002f. Characterization of Vadose Zone Sediment: Borehole 299-E33-46 Near $B$-110 in the B-BX-BY Waste Management Area. PNNL-14119, Pacific Northwest National Laboratory, Richland, Washington.

Serne RJ, BN Bjornstad, DG Horton, DC Lanigan, CW Lindenmeier, MJ Lindberg, RE Clayton, VL LeGore, RD Orr, IV Kutnyakov, SR Baum, KN Geiszler, MM Valenta, and TS Vickerman. 2004a. Characterization of Vadose Zone Sediments Below the TX Tank Farm: Boreholes C3830, C3831, C3832 and RCRA Borehole 299-W10-27. PNNL-14594, Pacific Northwest National Laboratory, Richland, Washington.

Serne RJ, BN Bjornstad, DG Horton, DC Lanigan, HT Schaef, CW Lindenmeier, MJ Lindberg, RE Clayton, VL LeGore, KN Geiszler, SR Baum, MM Valenta, IV Kutnyakov, TS Vickerman, RD Orr, and CF Brown. 2004b. Characterization of Vadose Zone Sediments Below the T Tank Farm: Boreholes C4104, C4105, 299-W10-196, and RCRA Borehole 299-W11-39. PNNL-14849, Pacific Northwest National Laboratory, Richland, Washington.

Shannon RD. 1976. "Revised effective ionic-radii and systematic studies of interatomic distances in halides and chalcogenides". Acta Crystallographica Section A, 32, 751-767.

Slate, J. L. 1996. "Buried Carbonate Paleosols Developed in Pliocene-Pleistocene Deposits of the Pasco Basin, South-Central Washington, U.S.A.” Quat. Inter. 34-36: 191-196.

Slate, J. L. 2000. Nature and Variability of the Plio-Pleistocene Unit in the 200 West Area of the Hanford Site. BHI-01203, Rev. 0, Bechtel Hanford, Inc., Richland, Washington.

Smith, G. A. 1993. "Missoula Flood Dynamics and Magnitudes Inferred from Sedimentology of SlackWater Deposits on the Columbia Plateau." Geol. Soc. of Am. Bul. 195:77-100.

Smith, R.M., F.N. Hodges, and B.A. Williams. 2001. Groundwater Quality Assessment Plan for SingleShell Tank Waste Management Area U. PNNL-13612, Pacific Northwest National Laboratory, Richland, Washington.

Sobczyk SM. 2000. Subsurface Interpretation of the SX Tank Farm Hanford Site, Washington Based on Gamma-Ray Logging. Nez Perce Tribe Environmental Restoration Waste Management Program, Lapwai, Idaho. 
Tallman AM, KR Fecht, MC Marratt, and GV Last. 1979. Geology of the Separations Areas, Hanford Site, South-Central Washington. RHO-ST-23, Rockwell Hanford Operations, Richland, Washington.

USGS (United States Geological Survey). 2001. "Alkalinity and Acid Neutralizing Capacity." National Field Manual for the Collection of Water-Quality Data, $2^{\text {nd }}$ ed., eds. SA Rounds and FD Wilde. March 23, 2004 Available URL: http://water.usgs.gov/owq/FieldManual/Chapter6/section6.6/html/section6.6.htm

Wentworth, CK. 1922. "A Grade Scale and Class Terms for Clastic Sediments.” Journal of Geology, 30, p. 377-392.

Wood MI, TE Jones, R Schalla, BN Bjornstad, and FN Hodges. 2001. Subsurface Conditions Description of the T and TX-TY Waste Management Areas. HNF-7123, Rev. 0, CH2M HILL Hanford Group, Inc, Richland, Washington.

Wood, M.I, and T.E. Jones. 2003. Subsurface Conditions Description of the U Waste Management Area. RPP-15808, Rev. 0, CH2M HILL Hanford Group, Inc., Richland, Washington.

Zachara JM, SM Heald, BH Jeon, RK Kukkadapu, CX Liu, JP McKinley, AC Dohnalkova, and DA Moore. 2007. Reduction of pertechnetate [Tc(VII)] by aqueous $\mathrm{Fe}(\mathrm{II})$ and the nature of solid phase redox products. Geochimica et Cosmochimica Acta 71, 2137-2157 